UNITED STATES

DEPARTMENT OF THE INTERIOR

GEOLOGICAL SURVEY

THE QUALITY OF GROUND WATER IN THE PRINCIPAL AQUIFERS

OF NORTHEASTERN-NORTH CENTRAL WASHINGTON

By J. C. Ebbert

U.S. GEOLOGICAL SURVEY

Water-Resources Investigations Report 83-4102

Prepared in cooperation with the

STATE OF WASHINGTON DEPARTMENT OF ECOLOGY 


\section{UNITED STATES DEPARTMENT OF THE INTERIOR \\ WILLIAM P. CLARK, Secretary \\ GEOLOGICAL SURVEY \\ Dallas L. Peck, Director}

For additional information write to:

District Chief

U.S. Geological Survey

1201 Pacific Avenue - Suite 600

Tacoma, Washington 98402-4384
Copies of this report

can be purchased from:

Open-File Services Section Western Distribution Branch U.S. Geological Survey Box 25425, Federal Center Lakewood, Colorado 80225 (Telephone: (303) 234-5888) 
Abstract--

Introduction-_- 2

Purpose and scope-_-_ 2

Criteria for sampling-site selection-_- 2

Water-quality constituents and characteristics-_- 4

Maximum contaminant levels specified by U.S. Environmental

Protection Agency primary and secondary drinking water

regulations-_-_-_-_- 6

Significance of selected constituents and characteristics of water-- 7

Alkalinity-_- 7

Fecal-coliform bacteria-_-_- 8

Hardness-_-_- 8

Phenols-_- 9

Sodium-adsorption ratio-_- 9

Specific conductance-_-_- 10

Suitability of water for irrigation-_-_ 10

Explanation of geologic unit codes- 10

Data presentation-_-_ 10

Wel1- and spring-location numbering system-_-_-_- 12

Spokane Region--_-_- 13

Spokane Valley aquifer-_- 13

Description of the data base and previous investigations--- 13

Description of the aquifer-_ 15

Ground-water use-_-_- 15

Water quality- 15

Physical and major inorganic-chemical characteristics- 15

Trace elements-_- 21

Organic chemicals-_- 23

Coliform bacteria-_-_- 24

Airway Heights subregion-_- 24

Chamokane Creek subregion-_- 28

Northeastern Region-_- 30

Curlew-Sanpoil subregion-_- 30

Colville-Kettle subregion-- 30

Pend Oreille subregion- 35

Okanogan Region-- 37

Methow Region- 40

Chelan Region-- 43

Entiat-Wenatchee Region-- 46

Entiat subregion- 46

Cashmere subregion--_- 46

Selected references-- 50 


\section{ILLUSTRATIONS}

Page

FIGURE 1. Map of Washington State showing principal aquifer regions, as designated by Molenaar and others (1980), and five major regions, each containing approximately 100 sample sites--

2. Diagram showing the classification of irrigation waters- 11

3. Map of the Spokane Valley aquifer showing sample sites-- 14

4. Diagram showing the major ion percentages in water collected from Spokane Valley wells and springs, 1979- 16

5. Graph showing relation between dissolved-solids concentrations and specific-conductance values for Spokane Valley aquifer ground water--

6. Map showing average specific-conductance values of Spokane Valley aquifer ground water observed during the period May 1977 to May 1978, and graphs showing the history of specific-conductance variation for selected wells and springs-

7. Map showing average nitrate concentrations in Spokane Valley aquifer ground water observed during the period May 1977 to May 1978, and graphs showing the history of nitrate concentration variation for selected wells and springs

8. Map showing locations of Mead area wells, graphs of nitrate and chloride concentrations, and the direction of ground-water flow-

9. Map of the Airway Heights and Chamokane Creek subregions showing sample sites and average nitrate and dissolved-solids concentrations-_-

10. Diagram showing major ion percentages in water from the Airway Heights subregion wells-

11. Diagram showing major ion percentages in water from the Chamokane Creek subregion--

12. Map of the Curlew-Sanpoil subregion showing sample sites and average nitrate and dissolved-solids concentrations- - -

13. Diagram showing major ion percentages in water from the Curlew-Sanpoil region-

14. Map of the Colville-Kettle and Pend Oreille subregions showing sample sites and average nitrate and dissolved-solids concentrations

15. Diagram showing major ion percentages in water from the Colville-Kettle subregion--

16. Diagram showing major ion percentages in water from the Pend Oreille subregion- 
FIGURE 17. Map of the Okanogan Region showing sample sites and average nitrate and dissolved-solids concentrations--

18. Diagram showing major ion percentages in water from the Okanogan Region-_-_ 39

19. Map of the Methow Region showing sample sites and average nitrate and dissolved-solids concentrations-- 41

20. Diagram showing major ion percentages in water from the Methow Region-- 42

21. Map of the Chelan and Entiat-Wenatchee Regions showing sample sites and average nitrate and dissolved-solid concentrations-- 44

22. Diagram showing major ion percentages in water from the Chelan Region-_-_- 45

23. Diagram showing major ion percentages in water from the Entiat subregion-- 48

24. Diagram showing major ion percentages in water from the Cashmere subregion-- 49 
TABLE 1. Maximum contaminant levels specified by U.S. Environmental Protection Agency primary and secondary drinking water regulations--

2. Maximum alkalinity in waters used as a source of supply prior to treatment--

3. Physical, biological, and major chemical-constituent data for Spokane Valley ground water sampled during 1979

ummary of physical, biological, and major chemicalconstituent data for Spokane Valley ground water sampled during 1979

5. Summary of specific conductance values in ground-water samples from the Spokane Valley-

6. Summary of nitrate concentrations in ground-water samples from the Spokane Valley--

7. Trace-element concentrations in ground-water samples from the Spokane Valley-

8. Summary of trace-element concentrations in ground-water samples from the Spokane Valley--------------

9. Phenol and methylene-blue-active-substance
concentrations in ground-water samples from the
Spokane Valley--
10. Physical and major chemical-constituent data for
samples from the Airway Heights subregion-----
11. Summary of physical and major chemical-constituent

9. Phenol and methylene-blue-active-substance
concentrations in ground-water samples from the
Spokane Valley--
10. Physical and major chemical-constituent data for
samples from the Airway Heights subregion-----
11. Summary of physical and major chemical-constituent

9. Phenol and methylene-blue-active-substance
concentrations in ground-water samples from the
Spokane Valley--
10. Physical and major chemical-constituent data for
samples from the Airway Heights subregion--

9. Phenol and methylene-blue-active-substance
concentrations in ground-water samples from the
Spokane Valley--
10. Physical and major chemical-constituent data for
samples from the Airway Heights subregion-----
11. Summary of physical and major chemical-constituent

9. Phenol and methylene-blue-active-substance
concentrations in ground-water samples from the
Spokane Valley--
10. Physical and major chemical-constituent data for
samples from the Airway Heights subregion-data for ground-water samples from the Airway Heights subregion-

12. Trace-element concentrations in ground-water samples from the Airway Heights subregion----------------

13. Physical, biological, and major chemical-constituent data for ground-water samples from the Chamokane Creek subregion--_-_-_-_-_-_-_-_-_-_-_-_-_-_

14. Summary of physical, biological, and major chemicalconstituent data for ground-water samples from the Chamokane Creek subregion--_-_-_-_-_-_-_-_-_-_-_--

15. Trace-element concentrations in ground-water samples from the Chamokane Creek subregion--_-_---_-_---

16. Physical, biological, and major chemical-constituent data for ground-water samples from the Curlew-Sanpoil subregion---------------------------------

17. Summary of physical, biological, and major chemicalconstituent data for ground-water samples from the Curlew-Sanpoil subregion----_------_----------

18. Trace-element concentrations in ground-water samples from the Curlew-Sanpoil subregion 
TABLE 19. Physical, biological, and major chemical-constituent data for ground-water samples from the ColvilleKettle subregion------_---_---_----------- 88

20. Summary of physical, biological, and major chemicalconstituent data for ground-water samples from the Colville-Kettle subregion---_---_--_---------- 90

21. Trace-element concentrations in ground-water samples from the Colville-Kettle subregion--------------

22. Physical, biological, and major chemical-constituent data for ground-water samples from the Pend Oreille subregion------------

23. Summary of physical, biological, and major chemicalconstituent data for ground-water samples from the Pend Oreille subregion--_-_-_-

24. Trace-element concentrations in ground-water samples from the Pend Oreille subregion---

25. Physical, biological, and major chemical-constituent data for ground-water samples from the Okanogan Region-

26. Summary of physical, biological, and major chemica1-
constituent data for ground-water samples from the
Okanogan Region--
27. Trace-element concentrations in ground-water samples
from the Okanogan Region--
28. Physica1, biological, and major chemical-constituent
data for ground-water samples from the Methow Region

26. Summary of physical, biological, and major chemica1-
constituent data for ground-water samples from the
Okanogan Region--
27. Trace-element concentrations in ground-water samples
from the Okanogan Region--
28. Physica1, biological, and major chemical-constituent
data for ground-water samples from the Methow Region-

26. Summary of physical, biological, and major chemica1-
constituent data for ground-water samples from the
Okanogan Region---
27. Trace-element concentrations in ground-water samples
from the Okanogan Region-----
28. Physica1, biological, and major chemical-constituent
data for ground-water samples from the Methow Region

26. Summary of physical, biological, and major chemical-
constituent data for ground-water samples from the
Okanogan Region---
27. Trace-element concentrations in ground-water samples
from the Okanogan Region-----
28. Physica1, biological, and major chemical-constituent
data for ground-water samples from the Methow Region

26. Summary of physical, biological, and major chemical-
constituent data for ground-water samples from the
Okanogan Region--
27. Trace-element concentrations in ground-water samples
from the Okanogan Region----
28. Physica1, biological, and major chemical-constituent
data for ground-water samples from the Methow Region-constituent data for ground-water samples from the Methow Region-----_-

30. Trace-element concentrations in ground-water samples from the Methow Region-----

31. Physica1, biological, and major chemical-constituent data for ground-water samples from the Chelan Region--

32. Physica1, biological, and major chemical-constituent data for ground-water samples from the Entiat subregion-------------------------------------

33. Summary of physical, biological, and major chemical constituent data for ground-water samples from the Entiat subregion--------

34. Trace-element concentrations in ground-water samples from the Entiat subregion--

35. Physical, biological, and major chemical-constitue
data for ground-water samples from the Cashmere subregion-----------

36. Summary of physical, biological, and major chemicalconstituent data for ground-water samples from the

37. Trace-element concentrations in ground-water samples from the Cashmere subregion-- 
METRIC CONVERSION FACTORS

Multiply

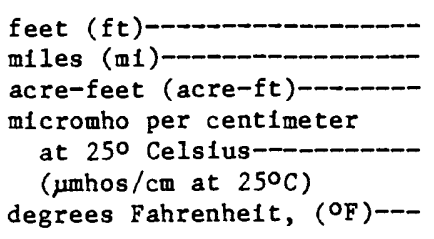

By

0.3048

1.609

0.001233

1.000

0.5556 , after subtracting 32 degrees
To obtain

meters (m)

kllometers ( $\mathrm{km})$

cublc hectometers ( $\mathrm{hm}^{3}$ )

microsfemen per centimeter at 250 Celsius $\left(\mu \mathrm{S} / \mathrm{cm}\right.$ at $25^{\circ} \mathrm{C}$ ) degrees Celsius $\left({ }^{\circ} \mathrm{C}\right)$

OTHER CONVERSION FACTORS

National Geodetic Vertical Datum of 1929 (NGVD of 1929): A geodet1c datum derlved from a general adjustment of the first-order level nets of both the United States and Canada, formerly called mean sea level. NGVD of 1929 is referred to as sea level in the text of this report and as NGVD in automatic-data-processed tables. 


\title{
THE QUALITY OF GROUND WATER IN THE PRINCIPAL AQUIFERS \\ OF NORTHEASTERN-NORTH CENTRAL WASHINGTON
}

By J. C. Ebbert

\begin{abstract}
The quality of ground water in major aquifers in northeastern-north central Washington was assessed in terms of inorganic chemical, trace-metal, and fecal-coliform concentrations. For the Spokane Valley aquifer some organic chemical data were also included. Results of this assessment indicate that the ground water in the region is generally suitable for most uses.

With some exceptions, ground water in the region can be characterized as moderately hard to hard calcium-magnesium-bicarbonate-type water. Median nitrate concentrations ranged from 0.14 to 2.4 milligrams per liter. Constituent concentrations that exceeded limits recommended by U.S. Environmental Protection Agency secondary drinking water regulations were found in ground-water samples from 8 of the 11 aquifers sampled; however, the incidence of such samples was sporadic and did not reflect general ground-water-quality degradation. Iron concentrations in excess of 300 micrograms per liter or manganese concentrations in excess of 50 micrograms per liter constituted most exceedences of secondary regulations.

Except for two samples, concentrations of trace metals in the ground water of the region were below maximum contaminant levels specified by U.S. Environmental Protection Agency primary drinking water regulations. Nitrate concentrations in a few samples exceeded maximum contaminant levels, but these samples did not represent typical conditions.
\end{abstract}




\section{INTRODUCTION}

\section{Purpose and Scope}

The Washington State Department of Ecology requested the cooperative assistance of the U.S. Geological Survey in appraising the chemical quality of water in principal aquifers in the State. This resulted in a 5-year program, initiated in 1979 , to obtain and analyze water samples for chemical quality from 500 wells and springs in principal aquifers throughout the State. To apportion the wells and springs uniformly across the State, it was divided into five major regions (fig. l), and each major region was allocated 100 wells and springs. The selection of principal aquifers in each major region was based on the delineation of principal aquifers in Washington by Molenaar and others (1980).

The purpose of this report is to describe ground-water quality in the northeastern-north central region, a major region which includes the aquifer regions Spokane, Northeastern, Okanogan, Methow, Chelan, and Entiat-Wenatchee as identified by Molenaar (fig. 1). Ground-water-quality samples were collected in the northeastern-north central region during 1979 , and data from those samples as well as historic ground-water-quality data are used in this report.

\section{Criteria for Sampling-Site Selection}

The number of ground-water-quality samples collected from a given aquifer was determined by a qualitative assessment of the availability, use, and economic importance of the water in that aquifer. Individual wells and springs were selected for sampling in order to provide a relatively uniform areal distribution of sites over an aquifer. Although some sites were located in areas of potential ground-water-quality degradation resulting from landfills, industrial sites, and other localized sources of pollution, the primary objective of sampling was to provide a general assessment of the overall ground-water quality in the major aquifers. 


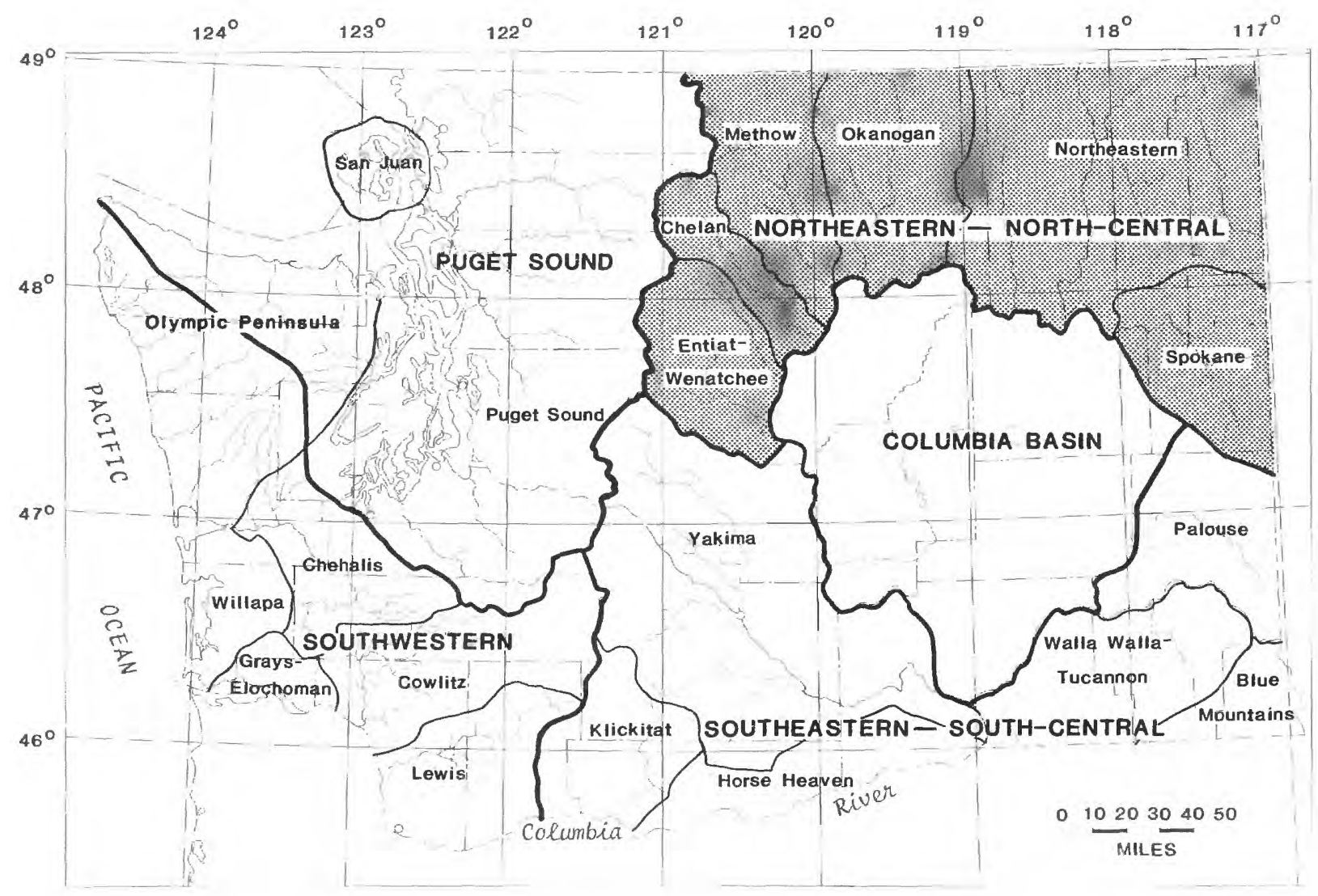

EXPLANATION

Boundary between major regions

Boundary between aquifer regions

small type - aquifer region

BOLD TYPE - major region

Shaded area is topic of this report

FIGURE 1.--Washington State showing principal aquifer regions, as designated by Molenaar and others (1980), and five major regions, each containing approximately 100 sample sites. 


\section{Water-Quality Constituents and Characteristics}

All samples that were collected during the summer of 1979 were analyzed for major anions and cations, nitrite-plus-nitrate, iron, and manganese. Hardness, sodium-absorption ratio, and dissolved-solids concentration were calculated from the results of the anion and cation determinations. Field measurements were made for temperature, specific conductance, and $\mathrm{pH}$ on all samples, and fecal-coliform-bacteria determinations were made on samples from domestic and public-supply wells. In addition, approximately 20 percent of the samples were analyzed for 11 trace elements: aluminum, arsenic, barium, cadmium, chromium, copper, lead, mercury, selenium, silver, and zinc.

Historic ground-water-quality data generally included the major anions and cations, nitrate, iron, and manganese. Trace-element and organic-chemical determinations were sporadic. In comparing the historic data with the 1979 data, some difficulties arise due to the changing of sample types and analytical methods. Prior to 1979, iron and manganese determinations were often made on unfiltered samples, and concentrations were reported as total recoverable. Samples collected in 1979 were filtered, and the corresponding concentrations were reported as dissolved. In samples with high iron concentrations there is often a precipitate of iron hydroxide that collects on the filter during filtration. In a raw, unfiltered sample much of this iron would be dissolved when the sample was preserved by acidification. Therefore, the concentration of iron in a filtered portion of a ground-water sample will often be less than the concentration in an unfiltered portion of the same sample.

There has also been little uniformity in the reporting of the concentration of inorganic nitrogen in water samples. Depending on the analysis, inorganic nitrogen concentrations have been reported as nitrate or as nitrite-plus-nitrate. Concentrations in filtered samples were reported as dissolved, whereas in raw, unfiltered samples they were reported as total. Furthermore, some nitrate concentrations have been reported as $\mathrm{NO}_{3}$ rather than as elemental nitrogen $\left(\mathrm{NO}_{3}-\mathrm{N}\right)$. In the basic data tables of this report (tables $3,10,13,16,19,22,25,28$, 31,32 , and 35 ) these data are presented as originally reported; however, in the summary tables (tables $4,11,14,17,20,23,26,29,33$, and 36) and on the figures, all nitrate and nitrite-plus-nitrate data are designated nitrate and expressed as $\mathrm{mg} / \mathrm{L}$ $\left(\mathrm{NO}_{3}-\mathrm{N}\right)$. This was done to facilitate comparison of data, and implies that for a given ground-water sample the various determinations will give similar results. For most ground-water samples this assumption is a satisfactory approximation. Determinations of nitrate and nitrite-plus-nitrate concentrations in a ground-water sample give approximately equivalent results because, in most ground water, little or no nitrite is present. 
Comparison of nitrate concentrations in filtered and unfiltered portions of the same sample becomes more complex. Here, one must evaluate the potential for the nitrate concentration in a sample to change during the period from collection to analysis. Two processes that could alter the nitrate concentration in a ground-water sample are nitrification and denitrification reactions. These reactions proceed slowly, if at all, without biological mediation, and sample filtration, which is done through a 0.45 -um filter, removes the microorganisms responsible for such mediation and effectively stabilizes a nitrate sample for the typical period between collection and analysis. This is not the case for an unfiltered sample, and unless it is treated with a biocide (until October 1980 this was not done), there is a potential for the nitrate concentration to change from the time of collection to analysis. Should this occur, nitrate determinations of the filtered and unfiltered components of the same sample would not give similar results; however, in many unfiltered ground-water samples little or no change in nitrate concentration will occur. Denitrification is unlikely because most samples contain oxygen either initially or after collection, and anaerobic conditions are the preferred environment for denitrifying bacteria. Furthermore, sufficient biodegradable carbon, a necessary energy source for denitrification, is usually not present in typical ground water (Viets and Hageman, 1971). Nitrification could occur, but in most ground water the concentration of reduced nitrogen compounds is low, minimizing the potential for nitrification reactions to significantly alter the initial nitrate concentration.

An additional complication in comparing recent and historic data is due to the changing of detection levels for trace-element determinations. For example, prior to 1979 many dissolved-lead determinations were done with a detection level of $100 \mathrm{ug} / \mathrm{L}$. After 1979, they were done with a detection level of $1 \mathrm{ug} / \mathrm{L}$. 
Maximum Contaminant Levels Specified By

U.S. Environmental Protection Agency

Primary and Secondary Drinking Water Regulations

U.S. Environmental Protection Agency national interim primary drinking water regulations apply to the physical and chemical characteristics of water that affect the health of consumers. They are applicable to public water supplies and are enforceable by the U.S. Environmental Protection Agency or the States. Primary drinking water regulations for constituents included in this report are given in table 1 .

National secondary drinking water regulations were also proposed by the U.S. Environmental Protection Agency. They apply to the esthetic qualities of drinking water and, unlike the primary regulations, are intended as guidelines and are not Federally enforceable. National secondary drinking water regulations for constituents and characteristics that are included in this report appear in table 1.

TABLE 1.--Maximum contaminant levels specified by U.S. Environmental
Protection Agency primary and secondary drinking water regulations

[U.S. Environmental Protection Agency, 1976, 1977.

Values in milligrams per liter unless otherwise noted]

\begin{tabular}{|c|c|}
\hline Contaminant & $\begin{array}{c}\text { Maximum } \\
\text { contaminant } \\
\text { level }\end{array}$ \\
\hline \multicolumn{2}{|c|}{ Primary Regulations } \\
\hline $\begin{array}{l}\text { Arsenic } \\
\text { Barium } \\
\text { Cadmium } \\
\text { Chromium } \\
\text { Lead } \\
\text { Mercury } \\
\text { Nitrate las N) } \\
\text { Selenium } \\
\text { Silver } \\
\text { Fluoride }\end{array}$ & $\begin{array}{c}0.05 \\
1 \\
.010 \\
.05 \\
.05 \\
.002 \\
10 \\
.01 \\
.05 \\
\text { a) } 1.4 \text { to } 2.4\end{array}$ \\
\hline
\end{tabular}

Secondary Regulations

\begin{tabular}{lc} 
Chloride & 250 \\
Copper & 1 \\
Foaming agents & .5 \\
Iron & .3 \\
Manganese & .05 \\
pH & $6.5-8.5$ units \\
Sulfate & 250 \\
Dissolved solids & 500 \\
Zinc & 5 \\
\hline
\end{tabular}

aDepends upon average daily air temperatures. 


\title{
Significance of Selected Constituents and
}

\author{
Characteristics of Water
}

The significance of selected water-quality constituents and characteristics not included in the U.S. Environmental Protection Agency primary and secondary drinking water regulations is discussed below. Although not included in the regulations, these constituents and characteristics are important in determining the suitability of water for domestic, industrial, or agricultural uses.

\section{Alkalinity}

Alkalinity is defined as the capacity of an aqueous solution to neutralize acid. Any ion that enters into a chemical reaction with strong acid can contribute to alkalinity; however, in most natural waters carbonate and bicarbonate ions are the principal components of alkalinity. The alkalinity of water used for domestic and municipal water supplies is important because it affects the amount of chemicals required for flocculation, softening, and control of corrosion in distribution systems. Generally, alkalinity resulting from naturally occurring materials is not a health hazard in drinking water, and alkalinities of natural waters rarely exceed 400 to $500 \mathrm{mg} / \mathrm{L}$ as $\mathrm{CaCO}_{3}$. For industrial applications, high alkalinity can be a problem in water used for food processing, especially where acidity is necessary for flavor and stability, such as in carbonated beverages. In some cases, alkalinity is desirable because of the corrosive properties of water with low alkalinity. Maximum alkalinities in source waters used for selected industrial purposes appear in table 2 .

TABLE 2.--Maximum alkalinity in waters used as a source of supply prior to treatment

[From U.S. Environmental Protection Agency, 1977b]

\begin{tabular}{|c|c|}
\hline Industry & $\begin{array}{l}\text { Alkalinity as } \mathrm{CaCO}_{3}, \\
\text { in milligrams per liter }\end{array}$ \\
\hline $\begin{array}{l}\text { Steam generation boiler makeup } \\
\text { Steam generation cooling } \\
\text { Textile mill products } \\
\text { Paper and all ied products } \\
\text { Chemical and allied products } \\
\text { Petroleum refining } \\
\text { Primary metal industries } \\
\text { Food canning industries } \\
\text { Bottled and canned soft drinks }\end{array}$ & $\begin{array}{r}350 \\
500 \\
50-200 \\
75-150 \\
500 \\
500 \\
200 \\
300 \\
85\end{array}$ \\
\hline
\end{tabular}


Fecal-coliform bacteria are nonpathogenic bacteria which normally inhabit the gut and feces of warmblooded animals. They are a subgroup of the total coliform group, which includes bacteria of nonfecal origin. The presence of fecal-coliform bacteria in water is an indicator of the contamination of the water supply by sewage or animal excrement. Since feces are known carriers of disease-producing bacteria, the contamination of a water supply as indicated by the presence of fecal-coliform bacteria can be a serious problem. Maximum contaminant levels for coliform bacteria in drinking water are specified by U.S. Environmental Protection Agency primary drinking water regulations in terms of total coliform bacteria, not fecal coliforms. Because the specification of these maximum contaminant levels is quite detailed, they are not included here. For the purpose of this report, it is sufficient to state that the presence of fecal-coliform bacteria in a water sample may indicate contamination of the source by sewage or animal excrement.

\section{Hardness}

The hardness of water is an important consideration for domestic, municipal, and industrial uses. It is related almost entirely to the presence of calcium and magnesium ions in water; however, other constituents, such as iron, manganese, and strontium, also contribute to hardness. The fraction of hardness which is equivalent to the alkalinity is called carbonate hardness, and any excess is called noncarbonate hardness. A classification of water by hardness content (Sawyer, 1960, p. 235) is as follows:

Hardness as $\mathrm{CaCO}_{3}$, in milligrams per liter

$$
\text { 0-75 }
$$

75-150

150-300

More than 300

\section{Description}

Soft

Moderately hard

Hard

Very hard 


\section{Phenols}

Phenolic compounds arise from numerous industrial sources and from the decomposition of naturally occurring organic substances. Phenol and most of its derivatives are not included in U.S. Environmental Protection Agency primary and secondary drinking water regulations; however, some of them are included in U.S. Environmental Protection Agency water quality criteria (1980). The criteria are not rules and have no regulatory impact, but they can provide guidance on the environmental effect of pollutants, which can be useful in deriving regulatory requirements.

The analytical method used for phenol determinations was the condensation of phenol with 4-aminoantipyrine, followed by oxidation under alkaline conditions to produce a colored compound, the intensity of which is proportional to the phenol concentration. This analytical method is sensitive to pure phenol and to many of its derivatives, making it difficult to compare the results of the analytical determinations for phenol with the water-quality criteria, which specify individual compounds. For example, U.S. Environmental Protection Agency water quality criteria (1980) recommend an ambient concentration limit for phenol of $3.5 \mathrm{mg} / \mathrm{L}$ in drinking water for the protection of human health. The recommended concentration limits for 2, 4, 6-trichlorophenol, a potential carcinogen, are $12 \mathrm{ug} / \mathrm{L}, 1.2 \mathrm{ug} / \mathrm{L}$, and $0.12 \mathrm{ug} / \mathrm{L}$, respectively, for incremental increases of cancer risk over a lifetime estimated at $10^{-5}, 10^{-6}$ and $10^{-7}$, respectively (U.S. Environmental Protection Agency, 1980). Because the analytical method is sensitive to both compounds, the identity of the specific phenolic compound, or compounds, is unknown and it is not possible to apply the water-quality criteria. Therefore, the phenol data included in this report serve only as an indicator for the presence of phenol and many of its derivatives.

\section{Sodium-Adsorption Ratio}

Excess sodium in irrigation water might become a problem because sodium enters into ion-exchange reactions with calcium or magnesium in the soil. This exchange process is undesirable because a build-up of sodium in the soil reduces its permeability and makes it difficult to cultivate. The adsorption of sodium from a given irrigation water is a function of the proportion of sodium to calcium and magnesium in the water. The sodium-adsorption ratio (SAR) is a measure of the degree to which sodium will be adsorbed by a soil from a given water when brought into equilibrium with it. It is defined as

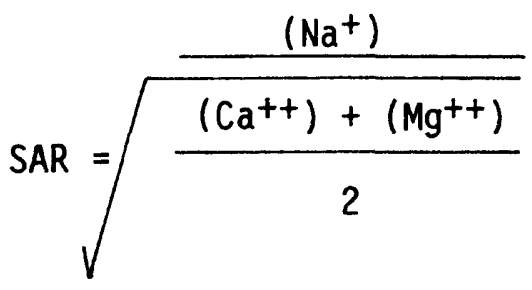

where ion concentrations are expressed as milliequivalents per liter. 


\section{Specific Conductance}

Specific conductance is a measure of the capacity of water to conduct an electrical current. It is commonly used as a measure of the mineral content of the water because it is the dissolved minerals that increase the water's current-carrying capacity.

\section{Suitability of Water for Irrigation}

The suitability of water for irrigation is in part determined by the degree of mineralization and the relative concentration of the minerals dissolved in water. The U.S. Department of Agriculture (1954) developed the diagram shown in figure 2, which uses specific conductance and sodium-adsorption ratio to determine the suitability of water for irrigation. Water is classified according to the sodium hazard and salinity hazard; $\mathrm{Cl}-\mathrm{Sl}$ water is low in both and, therefore, the best classification. The higher the numbers, the poorer the water for irrigation; C4-S4 is the poorest classification.

\section{Explanation of Geologic Unit Codes}

Most of the wells and springs sampled in the northeastern-north central region were completed in alluvial or glacial deposits, except in the Airway Heights subregion, where most wells were finished in basalt aquifers. Geologic unit codes appearing in the data tables are:

$$
\begin{aligned}
& \text { 110ALVM - Quaternary alluvium } \\
& \text { 112GLCV - Pleistocene glaciofluviatile } \\
& \text { 122CBRV - Columbia River Basalt Group }
\end{aligned}
$$

A few wells which were completed in geologic units other than those mentioned above were sampled. These wells are referenced in the data tables by a footnote.

\section{Data Presentation}

Physical, biological, and major chemical-constituent data are summarized for most of the principal aquifers. These summaries include maximum, minimum, and median values. In calculating a median value it is important to consider the bias resulting when a site is sampled more than once. To eliminate this bias, a mean concentration was computed for each constituent at sites with multiple samples, and the resulting mean was used in the calculation of median concentration in ground water of an aquifer.

Nitrate and dissolved-solids concentrations shown on the figures represent mean values if there was more than one sample for a given sample site. 


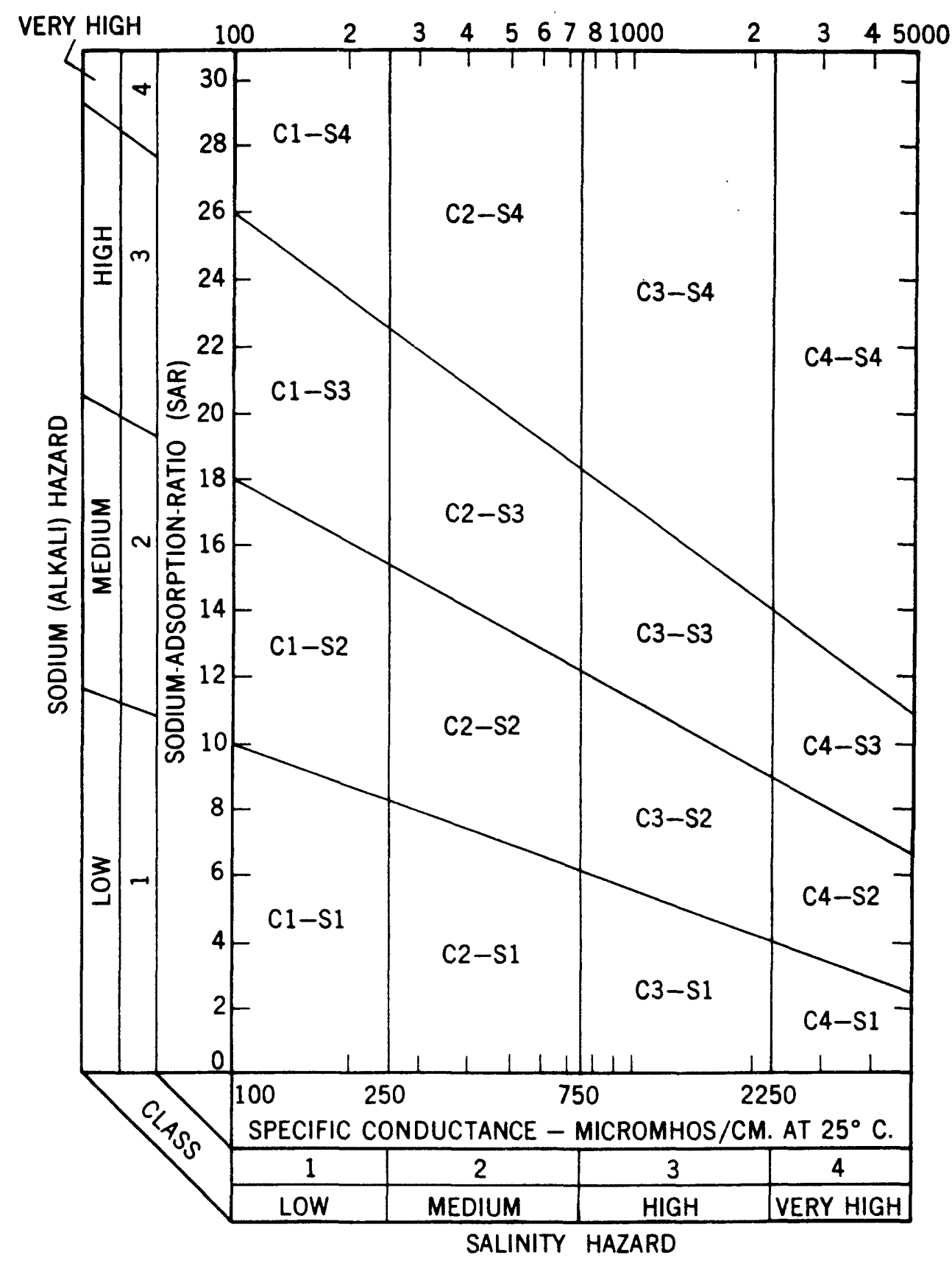

FIGURE 2.--Classification of irrigation waters

(U.S. Department of Agriculture, 1954). 
Wells in Washington are assigned numbers that identify their location in a township, range, and section. Well number 26/42-27 N 1 indicates, successively, the township (T.26 N.) and range (R.42 E.) north and east of the Williamette base line and meridian; the letters indicating north and east are omitted. The first number following the hyphen indicates the section (27) within the township, and the letter following the section gives the 40-acre subdivision of the section, as shown below. The number following the letter is the serial number of the well in the 40-acre subdivision. An " $\mathrm{s}$ " following the sequence number indicates that the site is a spring.

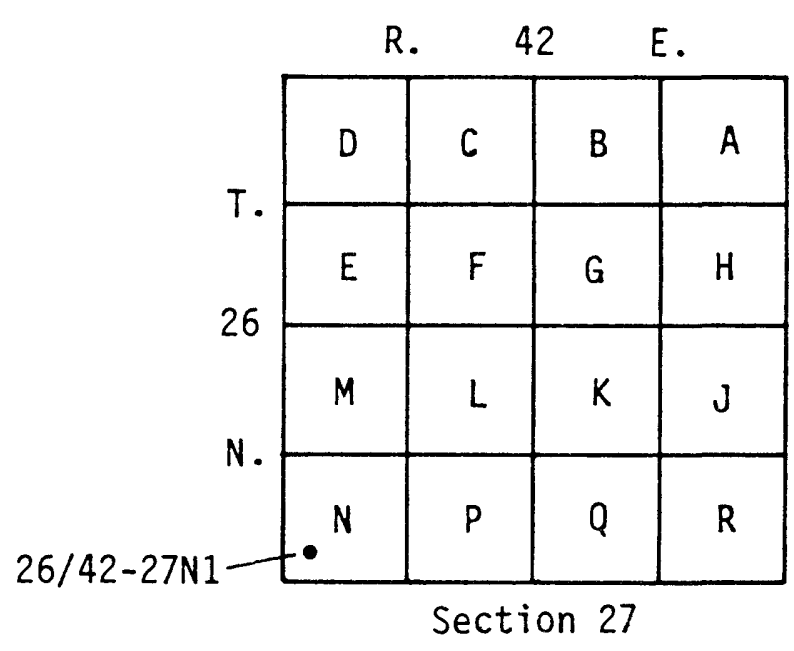




\section{SPOKANE REGION}

The Spokane Region includes most of Spokane County and small sections of Stevens, Lincoln, and Whitman Counties (fig. 1). The three principal aquifers in the region where ground water was sampled were the Spokane Valley aquifer, the basalt aquifers in the vicinity of Airway Heights, and the aquifer along Chamokane Creek. The Spokane Valley aquifer is shown in figure 3; the Airway Heights and Chamokane Creek subregions are shown in figure 9.

\section{Spokane Valley Aquifer}

\section{Description of the Data Base and Previous Investigations}

There has been much interest in the Spokane Valley aquifer due to its importance as a source of water for the city of Spokane and much of the surrounding area. The aquifer has been the subject of numerous investigations, some of which have been concerned with the quality of ground water in the aquifer. Because of these studies, the ground-water-quality data base for the Spokane Valley aquifer is extensive, compared with most other major aquifers in the State. It was therefore possible to present a more complete description of the quality of ground water in the Spokane Valley aquifer than for the other principal aquifers.

In this report the ground-water-quality data for the Spokane Valley aquifer include those collected from 22 wells and springs sampled during the summer of 1979 and other data previously collected, which are stored in WATSTORE, the U.S. Geological Survey's computer storage and retrieval system. Most of the historic data resulted from investigations conducted by the U.S. Geological Survey for, or in cooperation with, other agencies. Where possible, these data are summarized to reduce the size of the data tables. Additional data may be found in reports by Drost and Seitz (1978), Bolke and Vaccaro (1979), and Vaccaro and Bolke (1983).

Drost and Seitz (1978) describe the hydrologic characteristics of the Spokane Valley-Rathdrum Prairie aquifer in Washington and Idaho, the population distribution overlying the aquifer, the soils overlying the aquifer, the use and disposal of water withdrawn from the aquifer, and the quality of water in the aquifer. Drost and Seitz accumulated ground-water-quality data for about 1,200 samples from 400 sites from the files of Federal, State, and local agencies, from previous studies, and from private laboratories. Some of the data were tabulated in their report, and all of the data were included when they determined locations where constituent concentrations exceeded drinking water standards. Some information from their report is included in the following discussion for enhancement and clarification.

Bolke and Vaccaro (1979) tabulated hydrologic data from 1977 and 1978 for the Spokane Valley aquifer. Included were nitrogen, phosphorus, chloride, and specific-conductance data for ground-water samples. Vaccaro and Bolke (1983) evaluated the water-quality characteristics of the Spokane Valley aquifer using a solute-transport model. This report contains detailed information on the relation between the hydrologic characteristics of the aquifer and the quality of ground water in the aquifer. 


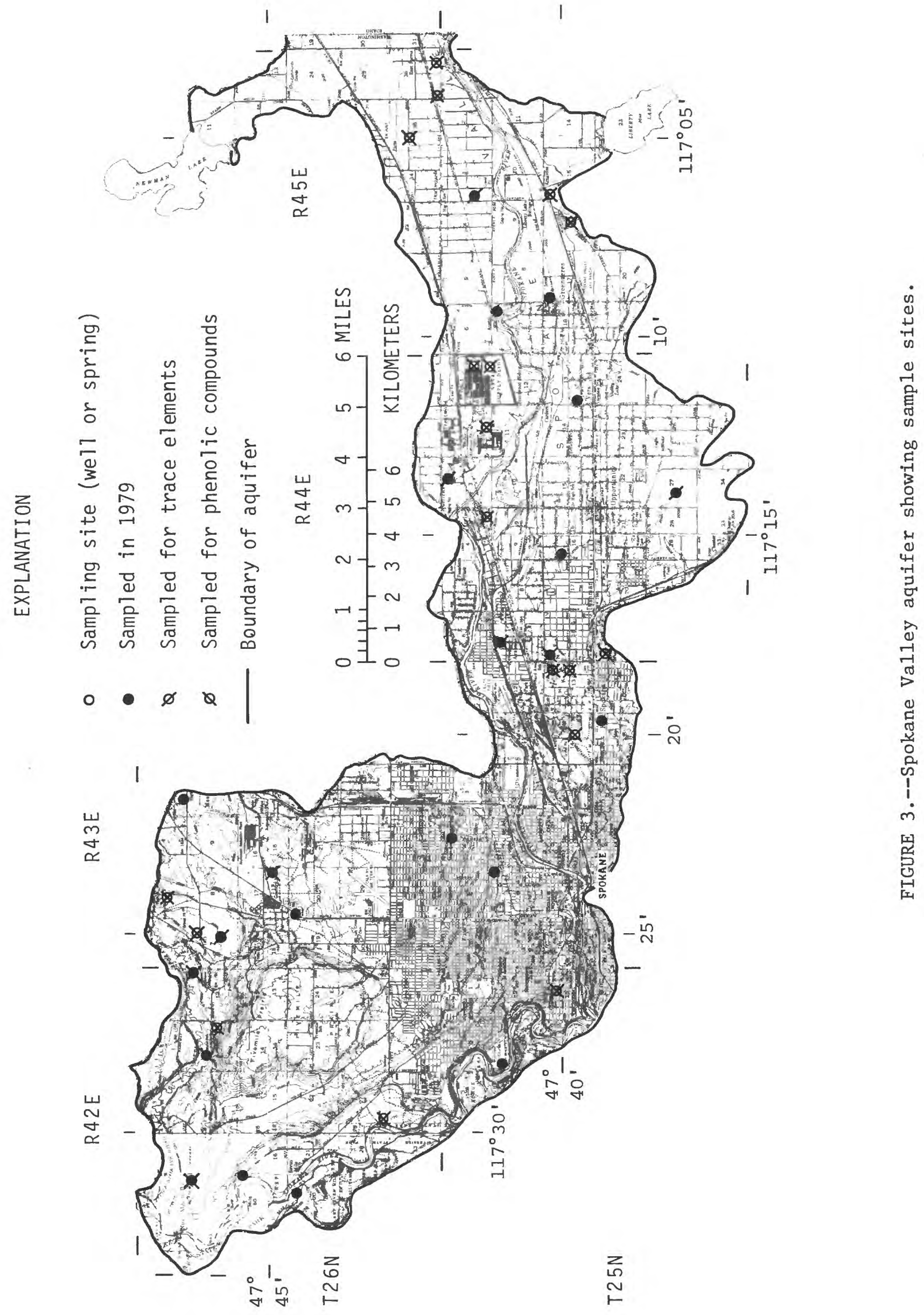


The boundaries of the Spokane Valley aquifer, as delineated by Drost and Seitz (1978), are shown in figure 3. Although the aquifer extends from Pend Oreille Lake, Idaho, to Long Lake, Wash., this report includes only the part of the aquifer that is in Washington. The aquifer is composed predominantly of Quaternary glaciofluvial deposits of unknown thickness, which lie above the consolidated Precambrian and Tertiary rocks that form the bottom and sides of the valley. Fivemile Prairie, a mesa northwest of the city of Spokane, consists of consolidated Tertiary deposits and separates the aquifer into the Hillyard Trough on the east and the lower Spokane River valley to the southwest.

\section{Ground-Water Use}

Water withdrawn from the Spokane Valley aquifer is used for municipal, domestic, irrigation, and industrial purposes. Most municipal use is for domestic and commercial purposes, and some water is used for irrigation and industry. In 1977, water used for municipal purposes was about 70 percent of the total withdrawn, or about 116,000 acre-feet, and water used for irrigation and industry was about 15 percent of the total, or about 24,000 acre-feet (Vaccaro and Bolke, 1983).

\section{Water Quality}

\section{Physical and major inorganic-chemical characteristics}

The physical and major inorganic-chemical data for samples from the 22 wells and springs sampled during the summer of 1979 appear in table 3 and are summarized in table 4. The percentage of major anions and cations in each of these samples is plotted on figure 4. Calcium and magnesium were the principal cations and bicarbonate was the principal anion in the samples. This is consistent with the findings of Drost and Seitz (1978) and Vaccaro and Bolke (1983).

The specific-conductance values of samples from 1979 and historic data for Spokane Valley aquifer wells and springs are summarized in table 5. Specific-conductance values ranged from 40 to 1,040 micromhos, with a median of 294 micromhos. The equation and graphical representation of the linear regression of dissolved-solids concentration on specific conductance are shown in figure 5 . Using the regression equation, the recommended drinking-water standard of 500 $\mathrm{mg} / \mathrm{L}$ of dissolved solids would correspond to a specific conductance of 916 micromhos. Well $25 / 45-16 \mathrm{Kl}$ is the only well in table 5 from which a sample had a specific conductance greater than 916 micromhos. Five water samples were collected from this well during the period 1973 to 1977. Dissolved-solids concentrations in these samples ranged from 445 to $594 \mathrm{mg} / \mathrm{L}$, with a mean of 492 $\mathrm{mg} / \mathrm{L}$. 


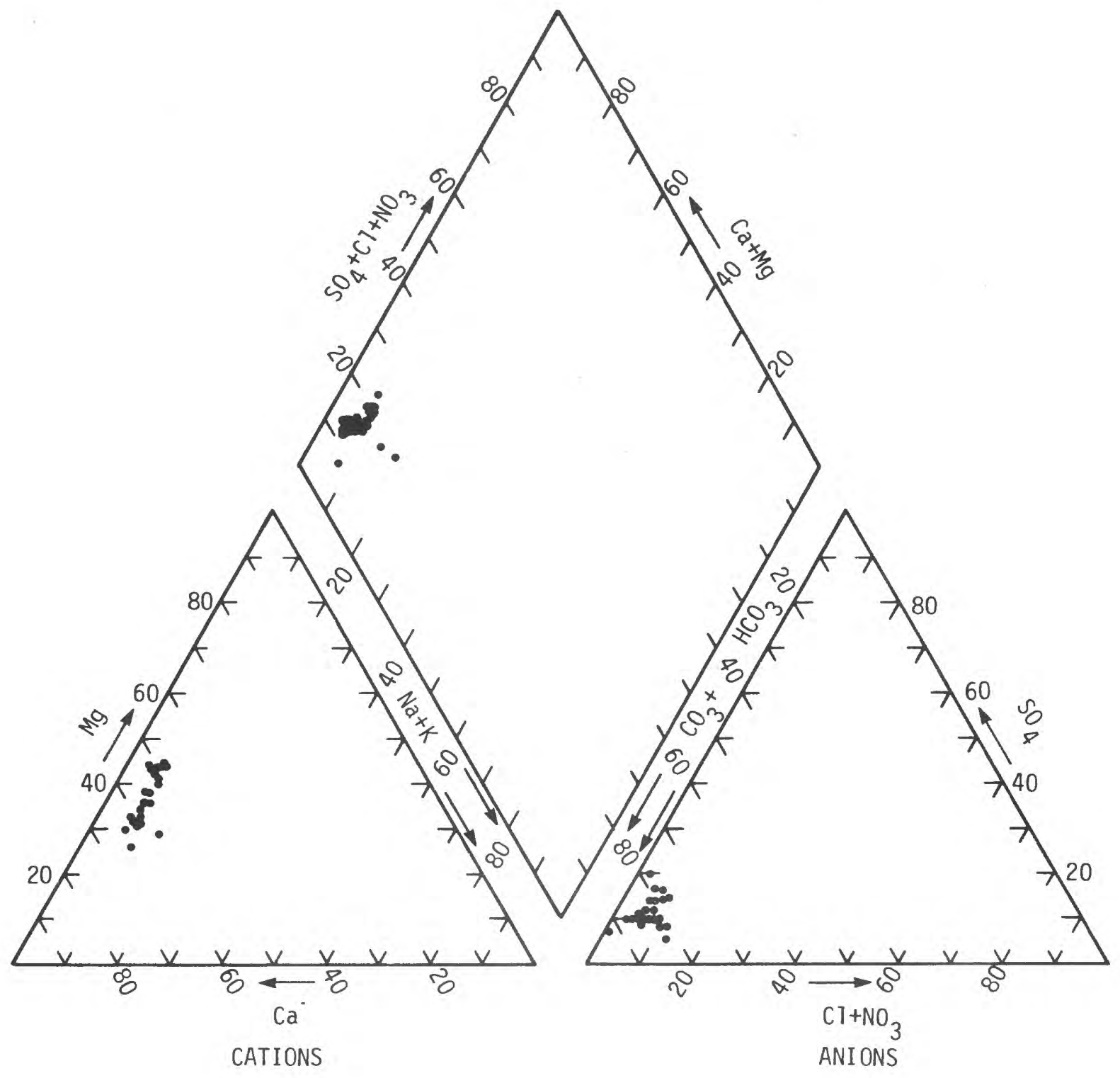

FIGURE 4.--Major ion percentages in water collected from Spokane Valley wells and springs, 1979. 


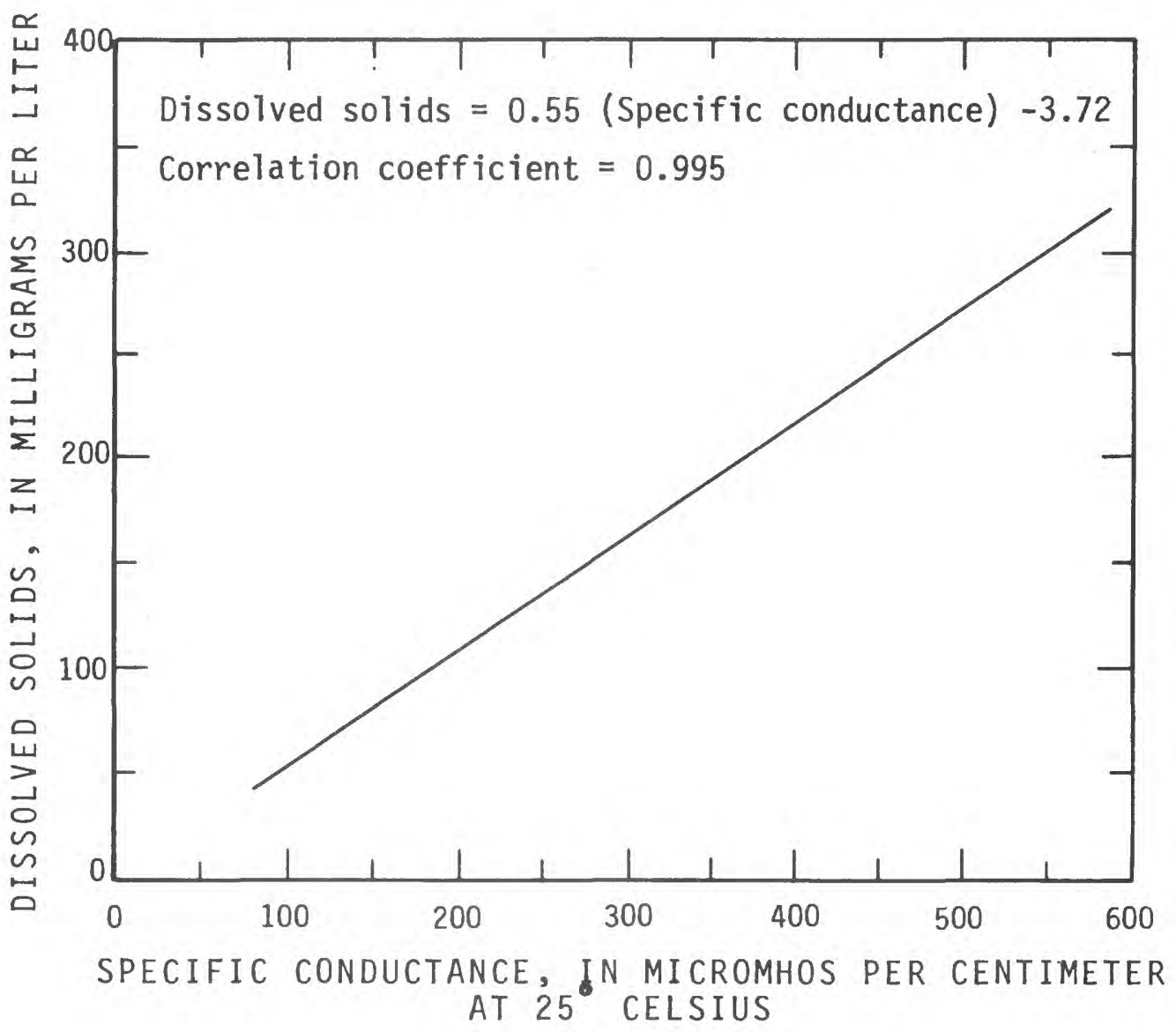

FIGURE 5.--Relation between dissolved-solids concentrations and specific-conductance values for Spokane Valley aquifer ground water. 
The areal variation of specific conductance values in Spokane Valley aquifer ground water is shown in figure 6. The contours represent average values resulting from three samplings conducted during 1977 and 1978. Specific-conductance values for the samples collected during 1979 are consistent with the variations shown in figure 6. Generally, specific-conductance values are lowest in the southeastern part of the aquifer and highest in the area around Mead, in the northern part of the aquifer. Also, the specific conductance of ground water is usually greater at the valley perimeter than adjacent to the Spokane River. Local increases in the specific conductance of ground water have resulted from land-use activities (Drost and Seitz, 1978, and Vaccaro and Bolke, 1983). For example, the specific conductance values and chloride concentrations in water from well 25/44-2QI were thought to have been affected by wastes from the Kaiser-Trentwood aluminum plant (Drost and Seitz, 1978). The specific-conductance values for water from this well range from 310 to 775 micromhos (table 4 ).

Specific-conductance values for wells with long-term records are shown in figure 6. No general long-term trend is evident. Because short-term fluctuations exceed long-term variations in water from wells such as $25 / 42-13 \mathrm{Bl}$, it is difficult to evaluate trends. Water from well 25/42-11El, which has the most complete long-term record, shows an apparent upward trend in specific-conductance values; however, these data must be interpreted with care. Instruments and methods of measurement have changed with time. Also, seasonal and climatic fluctuations in recharge to the aquifer, which influence ground-water quality, are not accounted for in the trend plots. Finally, the quality of water from wells such as 25/42-11E 1 , which are adjacent to the Spokane River, is influenced by recharge from the river. In such wells, changes in the quality of river water may affect the quality of water in the well more than land-use activities in the vicinity of the well.

Concentrations of $\mathrm{NO}_{3}-\mathrm{N}$ in water sampled historically and in 1979 from Spokane Valley aquifer wells and springs ranged from 0.00 to $9.2 \mathrm{mg} / \mathrm{L}$, with a median concentration of $1.3 \mathrm{mg} / \mathrm{L}$. Nitrate data are summarized in table 6. A plot prepared by Vaccaro and Bolke (1983) of the areal variation in nitrate concentrations is shown in figure 7. The lowest nitrate concentrations occur in the east and central parts of the aquifer, and higher concentrations are found in the peripheral areas.

$\mathrm{NO}_{3}-\mathrm{N}$ concentrations in water from wells and springs with relatively long-term records are shown in figure 7. As with specific- conductance values, it is difficult to determine trends. In many cases, seasonal or short-term variations exceed long-term variations.

Although none of the $\mathrm{NO}_{3}-\mathrm{N}$ concentrations exceeded the recommended limit for drinking water of $10 \mathrm{mg} / \mathrm{L}$, nitrate concentrations in water from several wells approached that limit. $\mathrm{A} \mathrm{NO}_{3}-\mathrm{N}$ concentration of $9.2 \mathrm{mg} / \mathrm{L}$ was found in a sample collected from well 25/43-23Al in February 1972. The mean $\mathrm{NO}_{3}-\mathrm{N}$ concentration in 20 samples collected from this well over a period from 1970 to 1979 was $2.8 \mathrm{mg} / \mathrm{L}$. This well was sampled monthly from September 1971 to September 1972, and except for the $\mathrm{NO}_{3}-\mathrm{N}$ concentration of $9.2 \mathrm{mg} / \mathrm{L}$ in the February sample, $\mathrm{NO}_{3}-\mathrm{N}$ concentrations ranged from 1.4 to $3.2 \mathrm{mg} / \mathrm{L}$. 


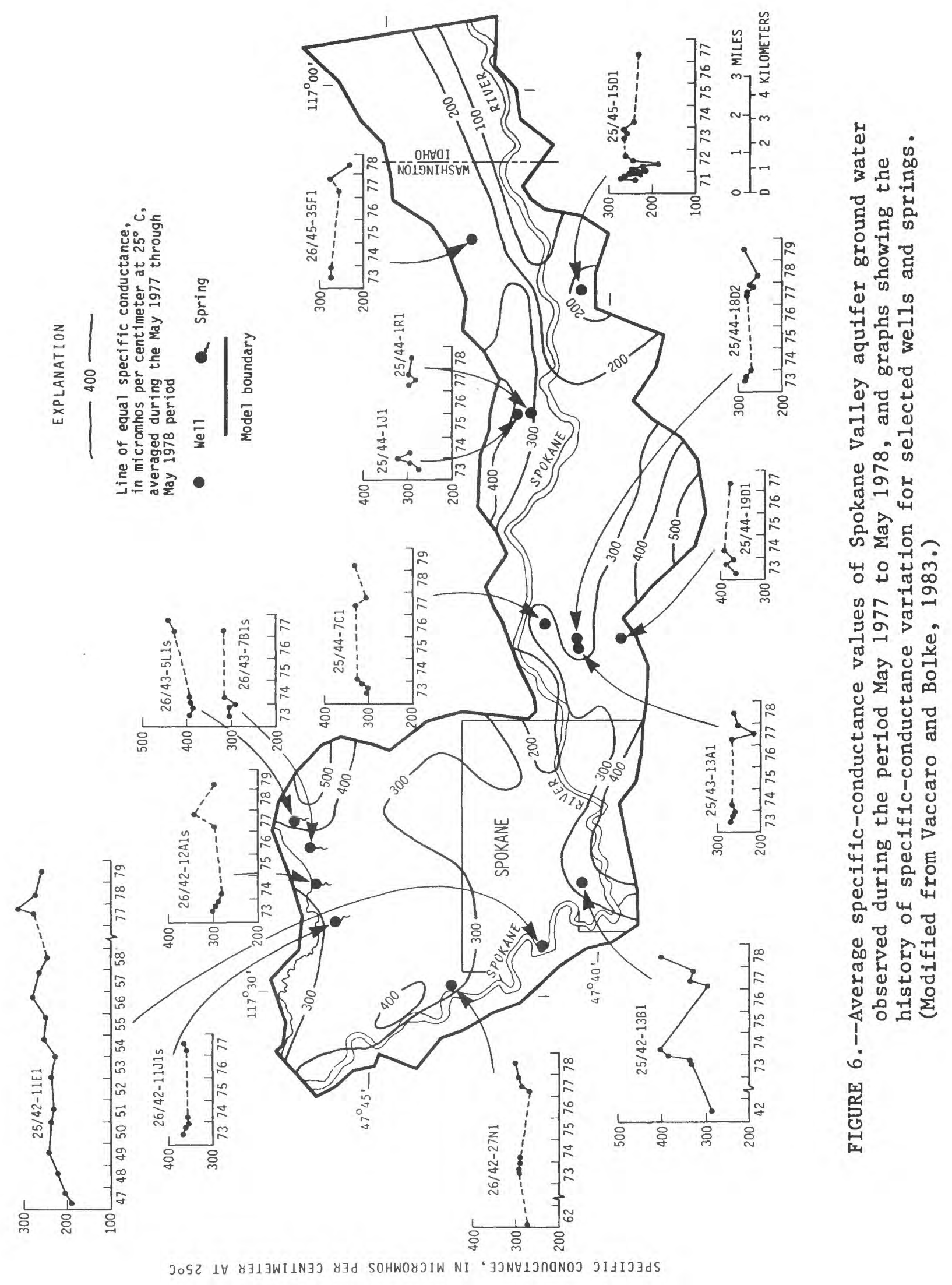



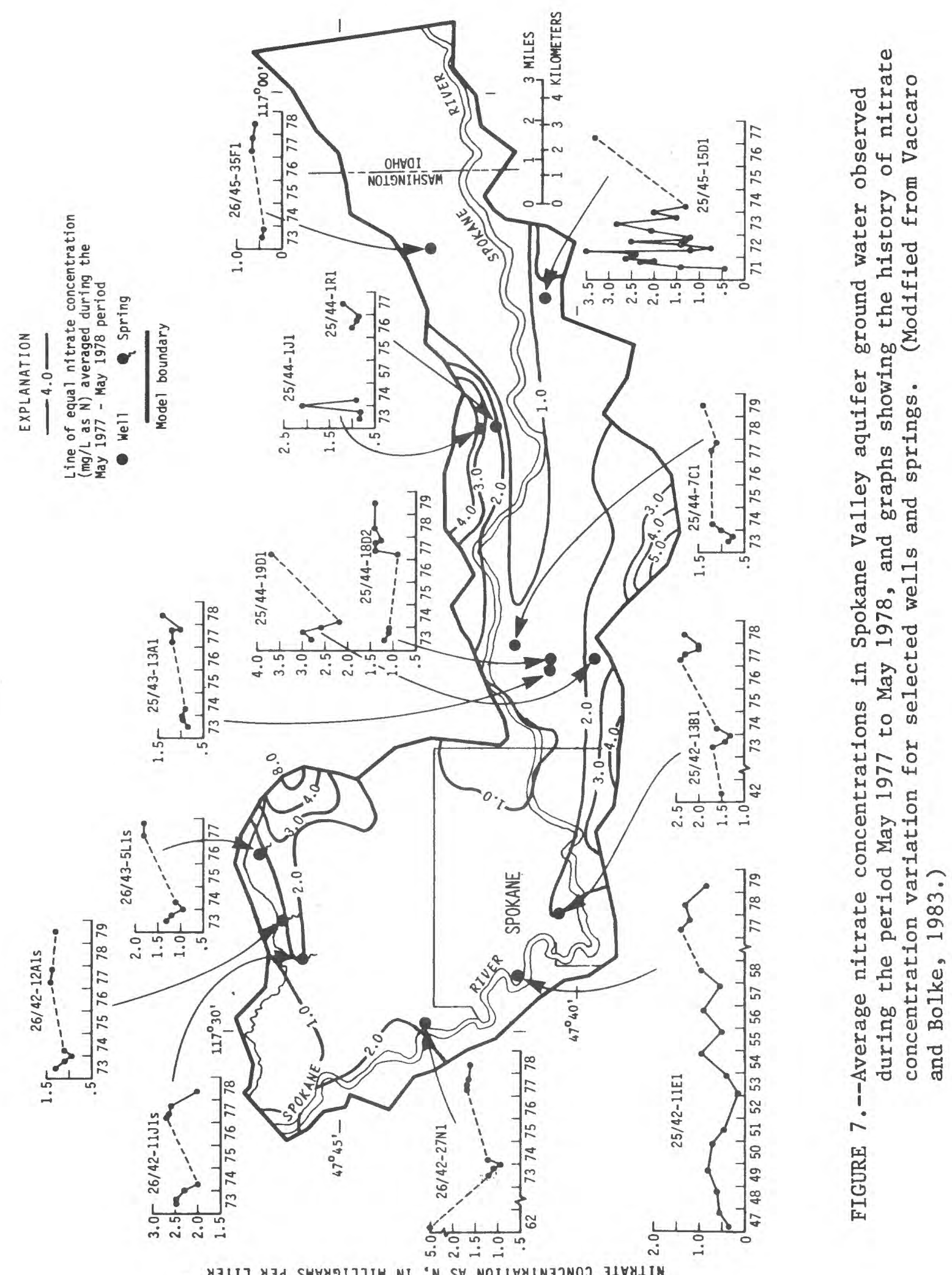
Water samples from wells $26 / 43-3 \mathrm{~N} 1,26 / 43-3 \mathrm{P} 1,26 / 43-8 \mathrm{~B} 4$, and $26 / 43-10 \mathrm{~K} 1$ in the Mead area had $\mathrm{NO}_{3}-\mathrm{N}$ concentrations exceeding $8 \mathrm{mg} / \mathrm{L}$. The locations and corresponding graphs of nitrate and chloride concentrations of these and several other wells in the vicinity of Mead are shown in figure 8. In their analysis of the flow and solute transport in the Spokane Valley aquifer, Vaccaro and Bolke (1983) attributed these high nitrate concentrations to high nitrate concentrations in the ground-water inflow from Peone Prairie mixing with ground water in the Spokane Valley aquifer. The source of the nitrate was not identified.

Support for their hypothesis lies in their analysis of ground-water flow in the Spokane Valley aquifer, ground-water inflow from Peone Prairie, and the correlation between chloride and nitrate concentrations in four Mead area wells (fig. 8). Examining their data collected during 1977 and 1978, Vaccaro and Bolke noted the correlation of high chloride and nitrate concentrations in water from wells $26 / 43-3 \mathrm{~N} 1,26 / 43-3 \mathrm{P} 1,26 / 43-8 \mathrm{~B} 4$, and $26 / 43-10 \mathrm{~K} 1$. This correlation is atypical of most water in the Spokane Valley aquifer, where the correlation coefficient between nitrate and chloride concentrations was 0.442 (Vaccaro and Bolke, 1983). The fluctuations in, and correlations between, nitrate and chloride concentrations in water from the four wells were thought to be caused by variations in the mixing between ground water in the Spokane Valley aquifer and ground-water inflow from Peone Prairie, where there was a common source of nitrate and chloride. Although well $26 / 43-8 G 2$ is in proximity to well $26 / 43-8 B 4$, it is in an area where the northward-flowing Spokane Valley aquifer water is just beginning to mix with the inflow from Peone Prairie, and $\mathrm{NO}_{3}-\mathrm{N}$ concentrations higher than $4 \mathrm{mg} / \mathrm{L}$ were not observed in water from this well.

High chloride concentrations that did not correlate with high nitrate concentrations were found in samples from wells 26/43-8G2, 26/43-16D2, and in the 1964 sample from well 26/43-8B4. Land-use activities that affected chloride, but not nitrate, concentrations were a possible cause.

Of the 22 samples collected during 1979, iron exceeded the recommended maximum concentration for drinking water of $300 \mathrm{ug} / \mathrm{L}$ in one sample, and manganese exceeded the recommended maximum concentration of $50 \mathrm{ug} / \mathrm{L}$ in two samples. Drost and Seitz (1978) found that iron concentrations exceeded the recommended concentration at 19 percent of the sites tested, and that manganese exceeded the recommended concentration at 6 percent of the sites tested.

Trace elements

Trace-element concentrations in ground-water samples from Spokane Valley aquifer wells and springs appear in table 7 and are summarized in table 8 . On two occasions trace-element concentrations in Spokane Valley aquifer ground-water samples exceeded the maximum contaminant levels specified by U.S. Environmental Protection Agency primary drinking water regulations. 


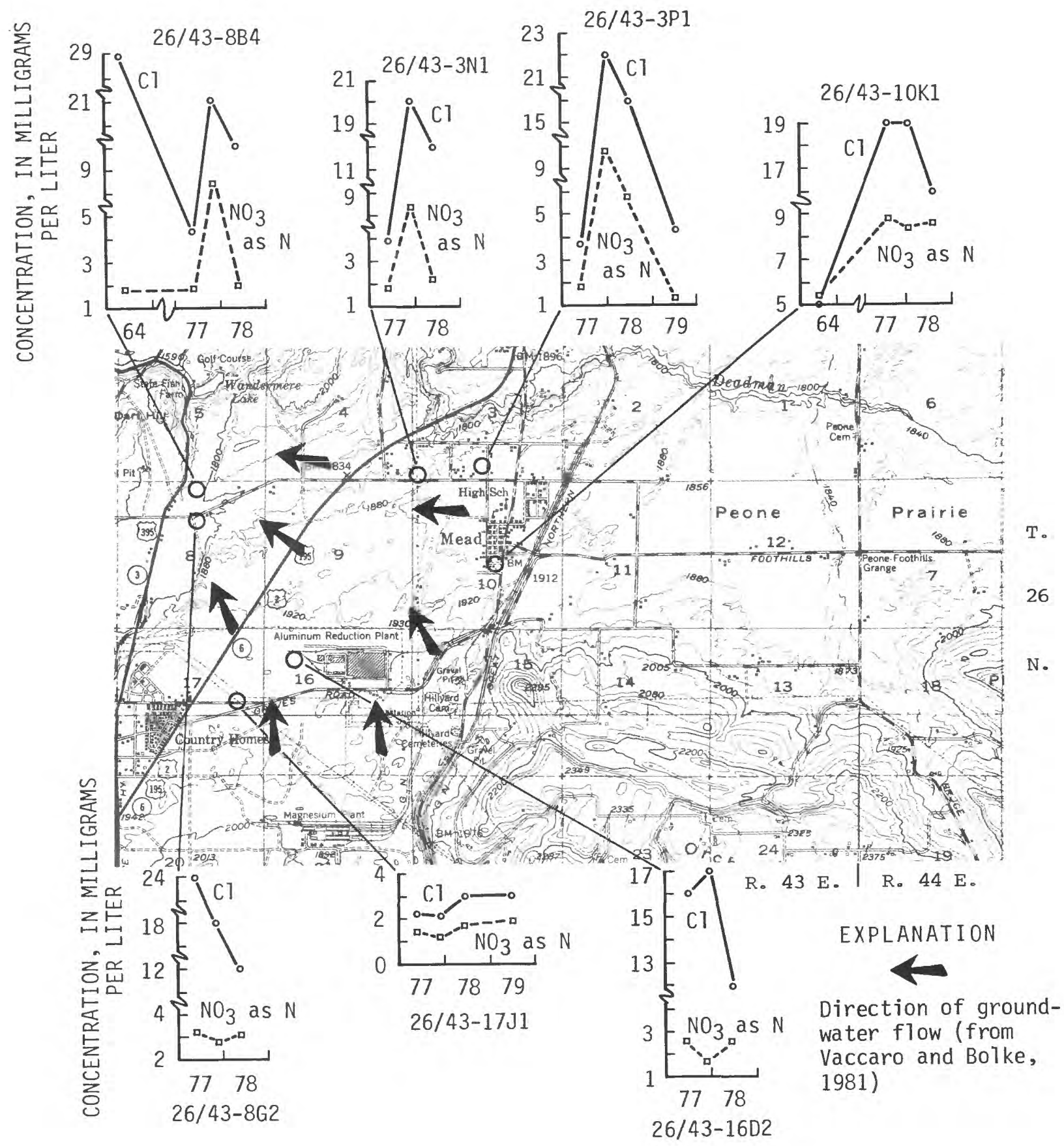

FIGURE 8.--Locations of Mead area wells, graphs of nitrate and chloride concentrations, and the direction of ground-water flow. 
A lead concentration of $61 \mathrm{ug} / \mathrm{L}$ in a sample collected in March 1977 from well 25/43-13Al exceeded the maximum contaminant level of $50 \mathrm{ug} / \mathrm{L}$. The median lead concentration in six samples collected from this well was $3.5 \mathrm{ug} / \mathrm{L}$. The concentration of copper in the March 1977 sample from the same well was $280 \mathrm{ug} / \mathrm{L}$, or 5.6 times the median concentration of $50 \mathrm{ug} / \mathrm{L}$ in samples from this well. A nearby well, 25/44-18D2, was sampled in April 1977, and no extraordinary lead or copper concentrations were observed.

An arsenic concentration of $64 \mathrm{ug} / \mathrm{L}$ in a sample collected on September 26, 1973, from the spring 26/43-7Bls exceeded the maximum contaminant level of 50 $\mathrm{ug} / \mathrm{L}$. This may be an isolated occurrence, as four other samples from this spring had concentrations ranging from 2 to $4 \mathrm{ug} / \mathrm{L}$ (table 7 ).

\section{Organic chemicals}

The use of phenolic compounds in some of the industries in the Spokane Valley prompted the Environmental Protection Agency to include phenol as one of the analytical determinations for ground-water samples collected from 1973 to 1977. These data, along with methylene-blue-active-substance (detergents) data, are given in table 9.

Primary and secondary drinking water regulations do not include phenol; however, the Environmental Protection Agency (1980) has issued water-quality criteria that include phenol and several phenolic compounds. Based on these criteria, the recommended limit for phenol in drinking water supplies is $3.5 \mathrm{mg} / \mathrm{L}$. There are difficulties in applying these criteria to the data because the analytical method that was used to measure the concentration of phenol in these samples was sensitive to pure phenol and to certain other phenolic compounds. The identity and concentration of individual phenolic compounds was not determined. The analysis for phenol is, however, useful as an indicator of phenolic contamination in ground water. This is especially true in light of the contamination by phenol of water from well 25/44-1 Jl (Drost and Seitz, 1978) where, in late 1975, a phenol concentration of $15 \mathrm{mg} / \mathrm{L}$ was detected. In samples taken from the well during 1973 and 1974, phenol was not detected (table 9). Phenol concentrations in samples from the Spokane Valley aquifer wells and springs listed in table 9 ranged from 0 to $12 \mathrm{ug} / \mathrm{L}$, with a median of $1.0 \mathrm{ug} / \mathrm{L}$.

The recommended limit for foaming agents (detergents) is $0.5 \mathrm{mg} / \mathrm{L}$ in drinking water. For samples from the wells listed in table 9, the concentrations ranged from 0.00 to $0.10 \mathrm{mg} / \mathrm{L}$, with a median of $0.01 \mathrm{mg} / \mathrm{L}$. 


\section{Coliform bacteria}

No fecal-coliform bacteria were detected in the samples collected from wells during the 1979 sampling. Fecal-coliform bacteria were present in the sample collected from spring 26/42-12Als (table 3), but the probability of sample contamination from a surficial source is high. Drost and Seitz (1978) summarized the potential for the contamination of Spokane Valley aquifer ground water by coliform bacteria. They concluded that, in most cases, positive tests for coliform bacteria in ground-water samples were the result of local contamination.

\section{Airway Heights Subregion}

The Airway Heights subregion located west of Spokane includes the vicinity of Airway Heights, Four Lakes, and Medical Lake (fig. 9). The principal ground-water reservoir of the subregion occurs in the lava flows, which constitute the Miocene Columbia River Basalt Group. In much of the area the basalt is only a few hundred feet thick or less, and is underlain by, or interlayered with, the Miocene Latah Formation, which is composed of fine silt and clay of low permeability. The Latah Formation was formed by the accumulation of fine-grained sediment that was deposited as the basalt flows dammed streams and formed lakes. The lowermost beds of the Latah Formation were deposited upon pre-Tertiary plutonic and metamorphic rocks that form the basement complex underlying the area. Generally, this formation is of low permeability and does not produce large quantities of ground water. Where the rocks are weathered or where fractures occur in unweathered rocks, permeability increases, and wells completed in such zones commonly have yields of about $10 \mathrm{gal} / \mathrm{min}$. Glacier-related deposits and extensive glacial flood sediments are found on the plains area north of Four Lakes. These deposits are relatively thin (less than $25 \mathrm{ft}$ ), and although these sediments would seem to be good potential aquifers, yields to wells are generally less than $25 \mathrm{gal} / \mathrm{min}$ due to low recharge.

Excluding wells for which records were not available, all wells sampled in the Airway Heights subregion were completed in basalt aquifers except for well $24 / 41 \mathrm{E}-23 \mathrm{Kl}$, which was terminated in the pre-Tertiary rock of the basement complex.

Ground-water quality in the Airway Heights subregion is generally adequate for most uses; however, $\mathrm{NO}_{3}-\mathrm{N}$ concentrations in samples from wells 25/41-10G1, $25 / 41-34 \mathrm{Cl}$, and 25/4l-35 $\mathrm{Cl}$ have exceeded the maximum contaminant level of 10 $\mathrm{mg} / \mathrm{L}$ specified in the U.S. Environmental Protection Agency primary drinking water regulations. Nitrate concentrations were consistently high in samples from these wells (see table 10). $\mathrm{NO}_{3}-\mathrm{N}$ concentrations in samples from well 24/41-3N 1 were erratic, ranging from 0.00 to $2.1 \mathrm{mg} / \mathrm{L}$. For the subregion in general, there was considerable areal variation of nitrate concentrations in ground water (fig. 9). 


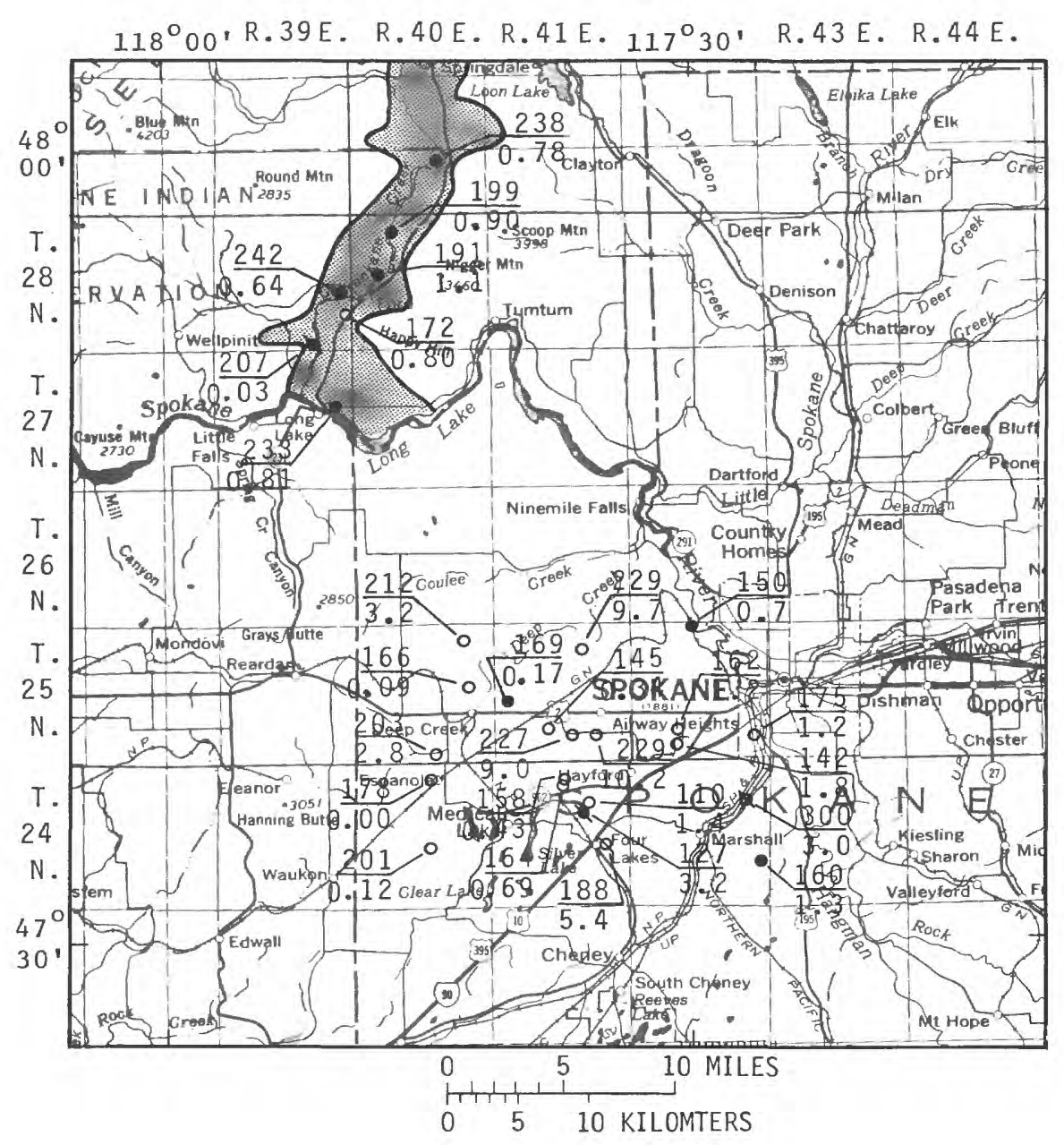

EXPLANATION

Area underlain by unconsolidated alluvial and glacial deposits in the vicinity of the sample sites.

Source: Huntting, M.T., and others, 1961, Geologic Map of Washington.
- Well sampled in 1979

- Well sampled prior to 1979

Q Well sampled for trace metals

201 dissolved solids, in $\mathrm{mg} / \mathrm{L}$ 0.12 nitrate as $\mathrm{N}$, in $\mathrm{mg} / \mathrm{L}$

FIGURE 9.--The Airway Heights and Chamokane Creek subregions showing sample sites and average nitrate and dissolved-solids concentrations. 
Percentages of anions and cations in ground-water samples from the Airway Heights subregion are plotted in figure 10. Some variations in anionic composition were due to variations in nitrate concentrations. Samples in which magnesium was the principal cation were approximately equal in number to those where calcium was the principal cation.

Physical and major chemical-constituent ground-water-quality data are summarized in table 11. Iron concentrations exceeded the recommended drinking-water limit of $300 \mathrm{ug} / \mathrm{L}$ at 4 of the 21 sample sites, and manganese concentrations exceeded the recommended limit of $50 \mathrm{ug} / \mathrm{L}$ in 2 of $15 \mathrm{samples}$. No other constituents, with the exception of nitrate, exceeded limits set by U.S. Environmental Protection Agency primary or secondary drinking water regulations.

Trace-element data for ground water in the subregion are sparse (table 12). A mercury concentration of $1.9 \mathrm{ug} / \mathrm{L}$ in a sample from well 24/41E-15A2 approaches the maximum contaminant level of $2.0 \mathrm{ug} / \mathrm{L}$ for drinking water. Additional trace-element data are needed to evaluate the ground-water quality of the subregion. 


\section{EXPLANATION}

- Samples collected in 1979

- Samples collected prior

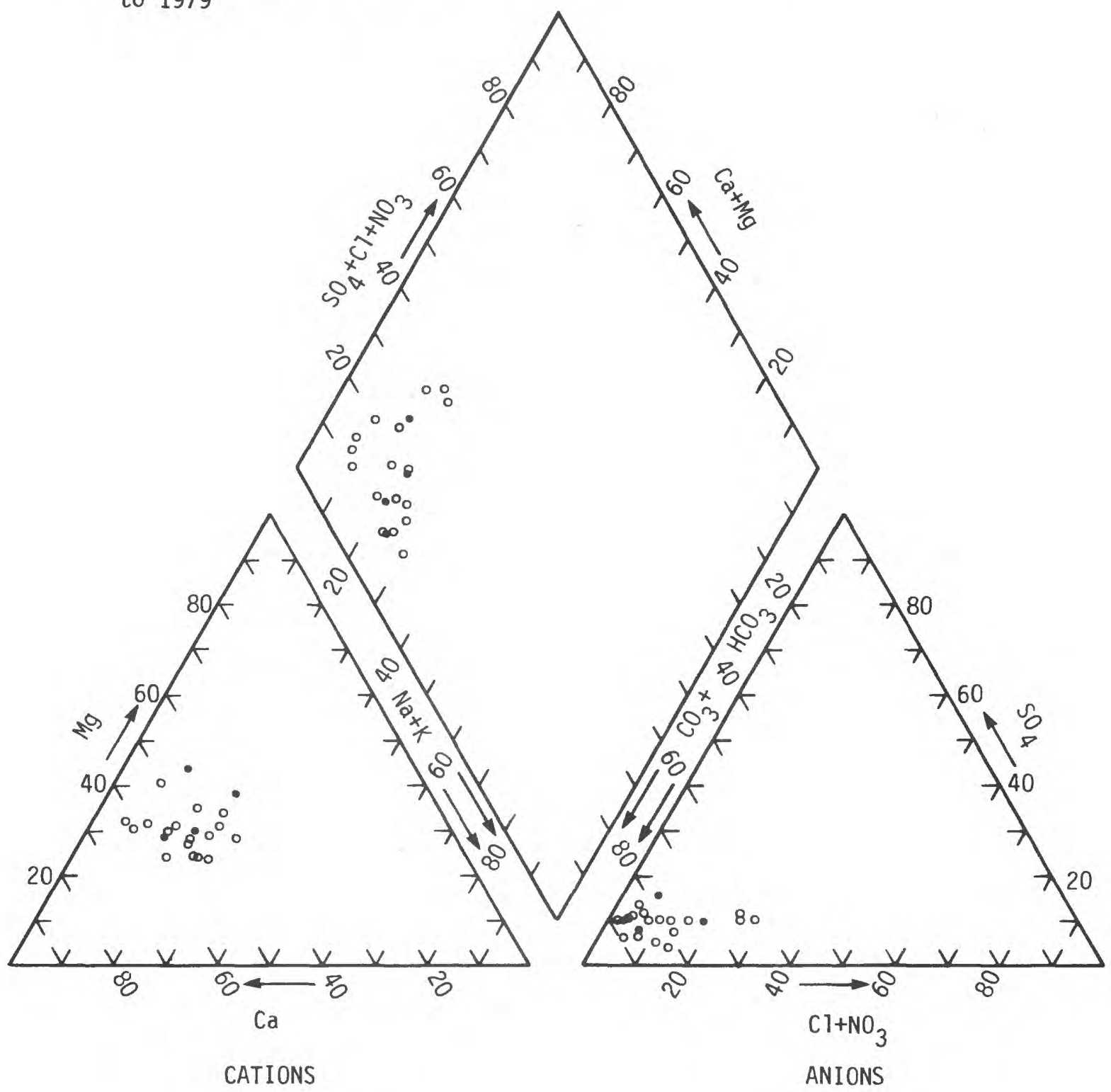

FIGURE 10.--Major ion percentages in water from the Airway Heights subregion wells. 


\section{Chamokane Creek Subregion}

Chamokane Creek originates in the Selkirk Mountains and flows into the Spokane River below Long Lake Dam. Alluvial and glacial deposits along the lower reach of Chamokane Creek constitute the third principal aquifer where ground water was sampled in the Spokane Region (fig. 9). The Chamokane Creek subregion is sparsely populated, and most wells are used to supply domestic and irrigation water.

The quality of the ground water sampled in the Chamokane Creek aquifer is such that the water is suitable for most uses (tables 13-15). $\mathrm{NO}_{3}-\mathrm{N}$ concentrations in ground-water samples ranged from 0.00 to $1.1 \mathrm{mg} / \mathrm{L}$, with a median concentration of $0.78 \mathrm{mg} / \mathrm{L}$. The major ions in the Chamokane Creek aquifer ground water were calcium, magnesium, and bicarbonate (fig. 11). The median dissolved-solids concentration was $207 \mathrm{mg} / \mathrm{L}$.

Iron concentrations in two of seven samples exceeded the recommended limit of $300 \mathrm{ug} / \mathrm{L}$ for drinking water. The median dissolved iron concentration in samples from the subregion was $220 \mathrm{ug} / \mathrm{L}$, indicating that iron concentrations are somewhat elevated compared with ground water from many of the other major aquifers. In general, manganese concentrations in the ground water sampled were low (median of $2 \mathrm{ug} / \mathrm{L}$; however, a manganese concentration of $90 \mathrm{ug} / \mathrm{L}$ in the sample from well 28/39-35Ll exceeded the recommended limit of $50 \mathrm{ug} / \mathrm{L}$ for drinking water. 
EXPLANTION

- Samples collected in 1979

- Samples collected prior to 1979

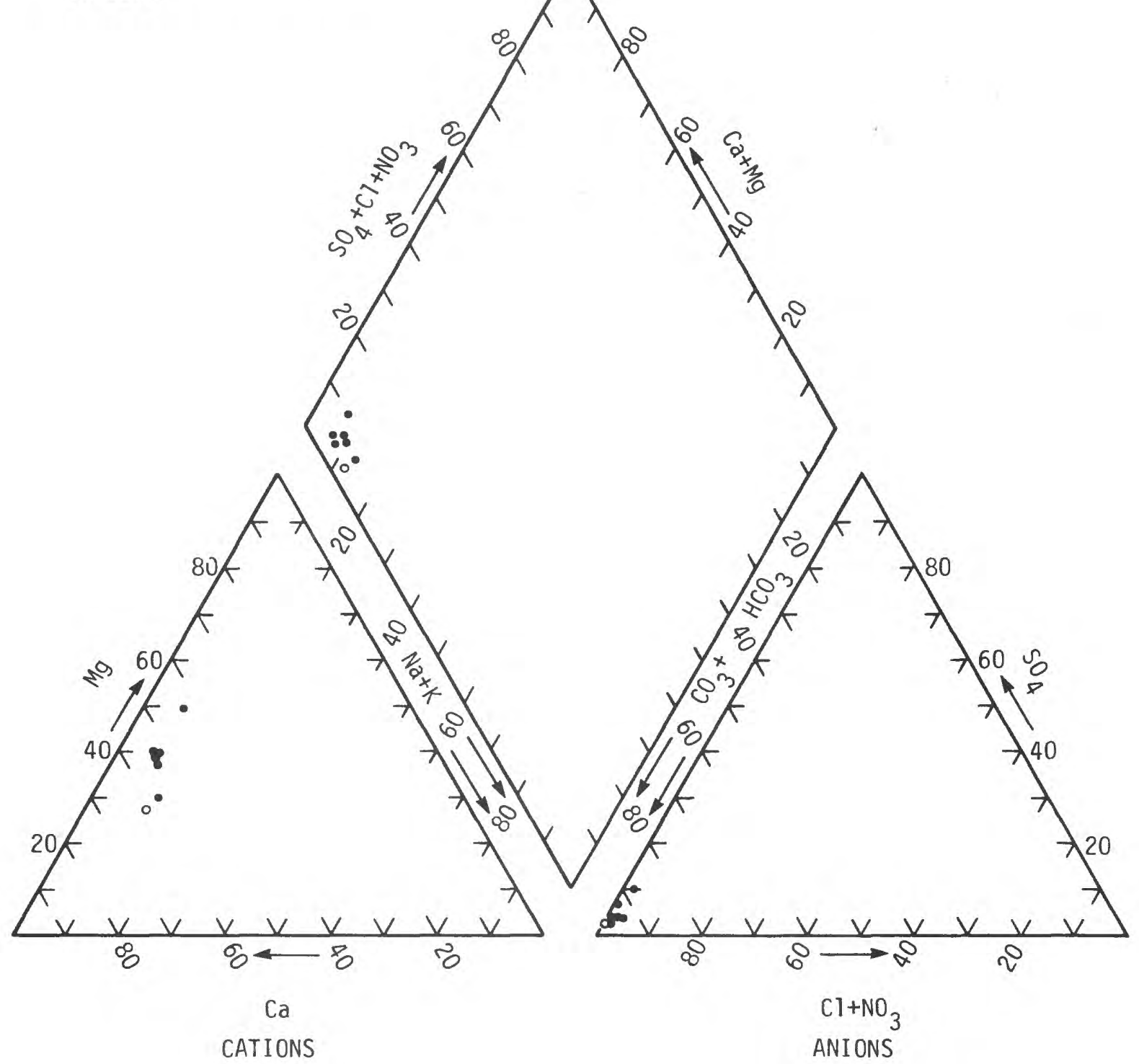

FIGURE 11.--Major ion percentages in water from the Chamokane Creek subregion wells. 


\section{NORTHEASTER N REGION}

The boundaries of the Northeastern Region are shown in figure 1. The region includes part of Okanogan County, all of Ferry County, and most of Stevens and Pend Oreille Counties. Ground-water sampling in the region was done in three areas where alluvial and glacial deposits form aquifer units-the Curlew-Sanpoil subregion, the Colville-Kettle subregion, and the Pend Oreille subregion (figs. 12 and 14).

\section{Curlew-Sanpoil Subregion}

The principal aquifers of the Curlew-Sanpoil subregion are glacial and alluvial deposits along the Sanpoil River and Curlew Creek and along the arc of the Kettle River from where it enters the United States near Ferry to where it flows back into Canada near Danville (fig. 12). The subregion is sparsely populated, and ground water is used mainly for domestic, municipal, and irrigation purposes.

Ground-water-quality data for the subregion appear in tables 16 and 18 and are summarized in table 17. On the basis of these data, the quality of ground water in the subregion is such that the water is suitable for most uses. All constituents were within drinking-water-regulation quidelines, except a total iron concentration of 570 $\mathrm{ug} / \mathrm{L}$ in water from well $29 / 33 \mathrm{E}-4 \mathrm{Ml}$. As shown in figure 13 , the principal cations in the samples were calcium and magnesium, and bicarbonate was the principal anion. Water from City of Republic wells $36 / 33 \mathrm{E}-7 \mathrm{~F} 1$ and $36 / 33 \mathrm{E}-7 \mathrm{~F} 2$ had a high percentage composition of sulfate compared with other samples from the subregion.

\section{Colville-Kettle Subregion}

Alluvial and glacial deposits along the Colville River and the lower reach of the Kettle River are the principal aquifers of the Colville-Kettle subregion. In 1979, five wells adjacent to the Colville River extending from Addy north to Colville were sampled, and two wells were sampled near Boyds adjacent to the Kettle River. Historical ground-water-quality data were collected near Chewela, near Colville, and adjacent to the Columbia River near the mouth of the Colville River (fig. 14).

The ground-water-quality data for the subregion appear in tables 19 and 21 and are summarized in table 20. Except for dissolved-manganese concentrations of 90 $\mathrm{ug} / \mathrm{L}$ in water from well $33 / 39 \mathrm{E}-13 \mathrm{Cl}$ and $290 \mathrm{ug} / \mathrm{L}$ in water from well $35 / 39 \mathrm{E}-10 \mathrm{Al}$, constituent concentrations in ground water sampled in the subregion were below maximum contaminant levels specified by U.S. Environmental Protection Agency primary and secondary drinking water regulations. Hardness values ranged from moderately hard to hard. The median $\mathrm{NO}_{3}-\mathrm{N}$ concentration was $0.41 \mathrm{mg} / \mathrm{L}$. City of Colville well 35/39E-10Al was sampled in 1960 and in 1979 , and on both occasions no nitrate was detected. Calcium and magnesium were the major cations and bicarbonate was the major anion in the ground-water sampled (fig. 15). For irrigation purposes, the ground water sampled had a low sodium hazard and a low to medium salinity hazard. 
EXPLANATION

Area underlain by unconsolidated alluvial and glacial deposits. Source: Huntting, M.T., and others, 1961, Geologic Map of Washington, and Molenaar, Dee, and others, 1980, Principal Aquifers and Well Yields in Washington.

- Well sampled in 1979

- Well sampled prior to 1979

Q Well sampled for trace metals 242 dissolved solids, in $\mathrm{mg} / \mathrm{L}$

1.3 nitrate as $\mathrm{N}$, in $\mathrm{mg} / \mathrm{L}$

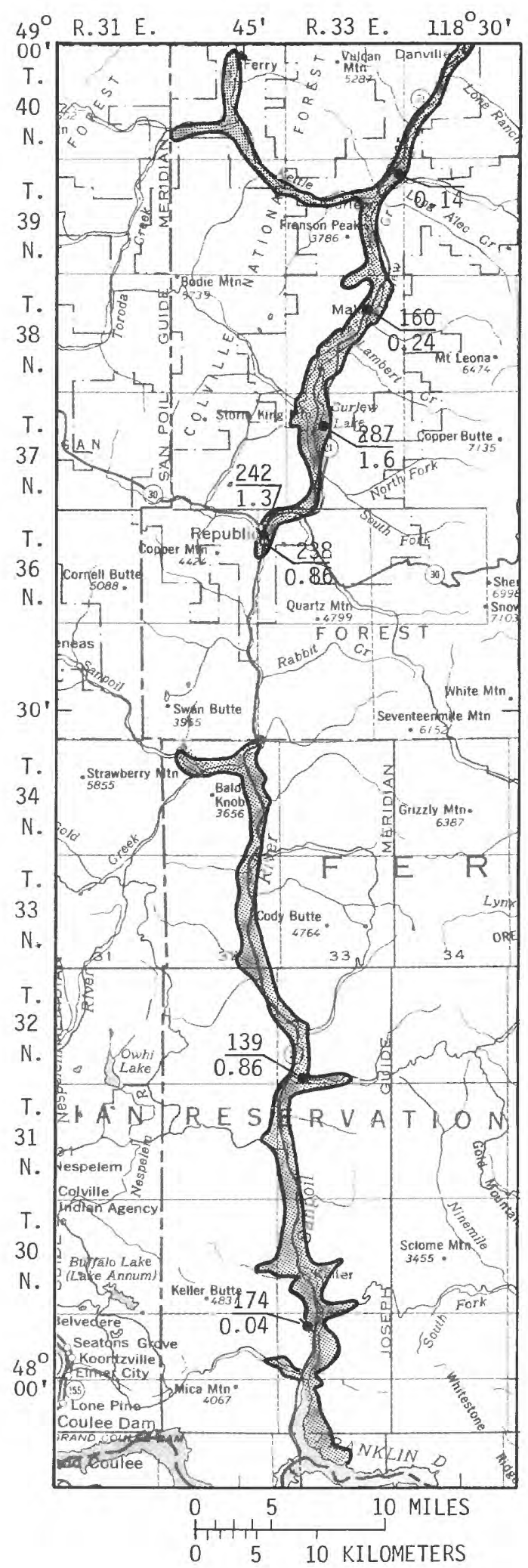

FIGURE 12.--Curlew-Sanpoil subregion showing sample sites and average nitrate and dissolved-solids concentrations. 
EXPLANATION

- Samples collected in 1979

- Samples collected prior to 1979

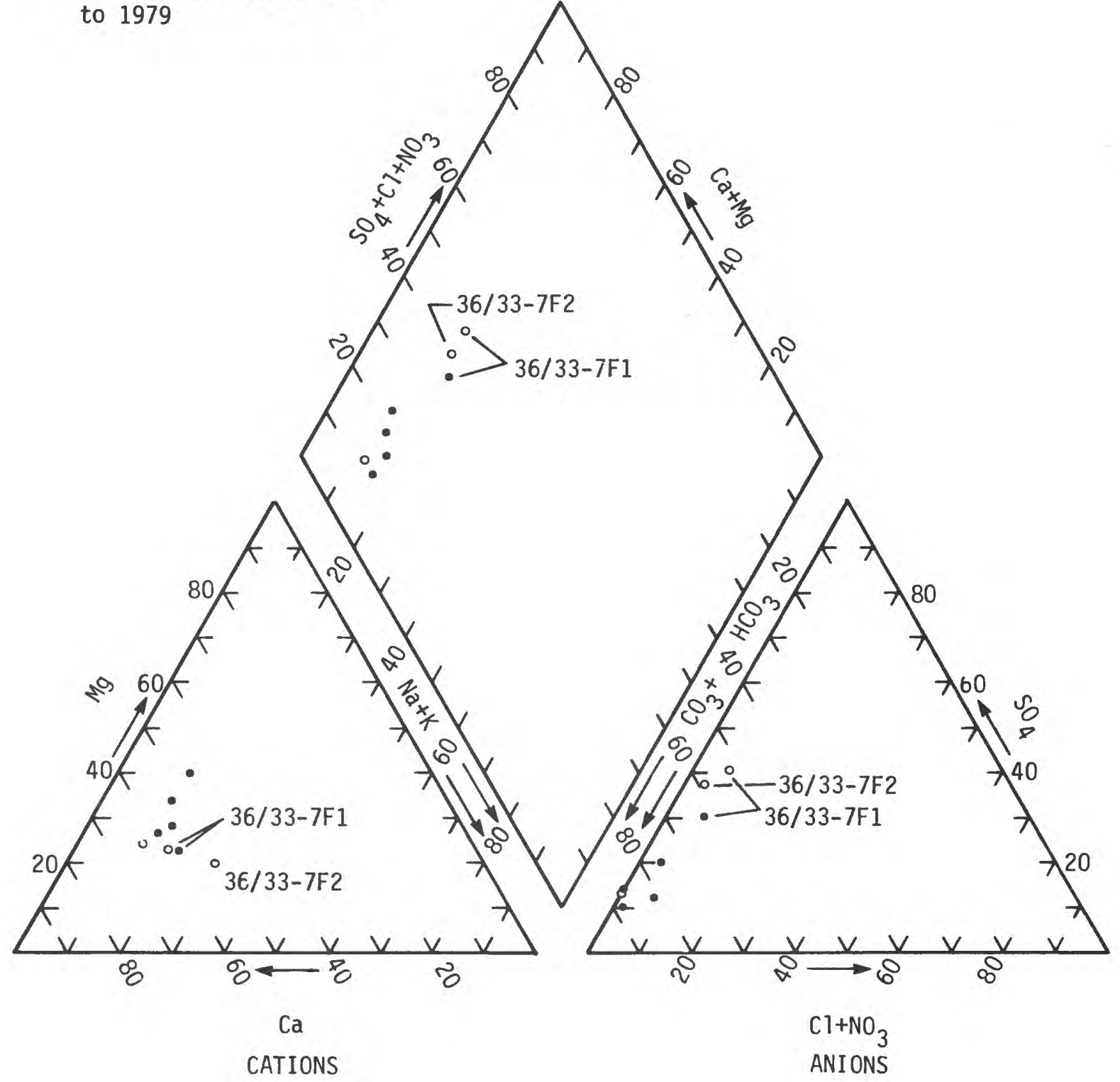

FIGURE 13.--Major ion percentages in water from the Curlew-Sanpoil subregion wells. 


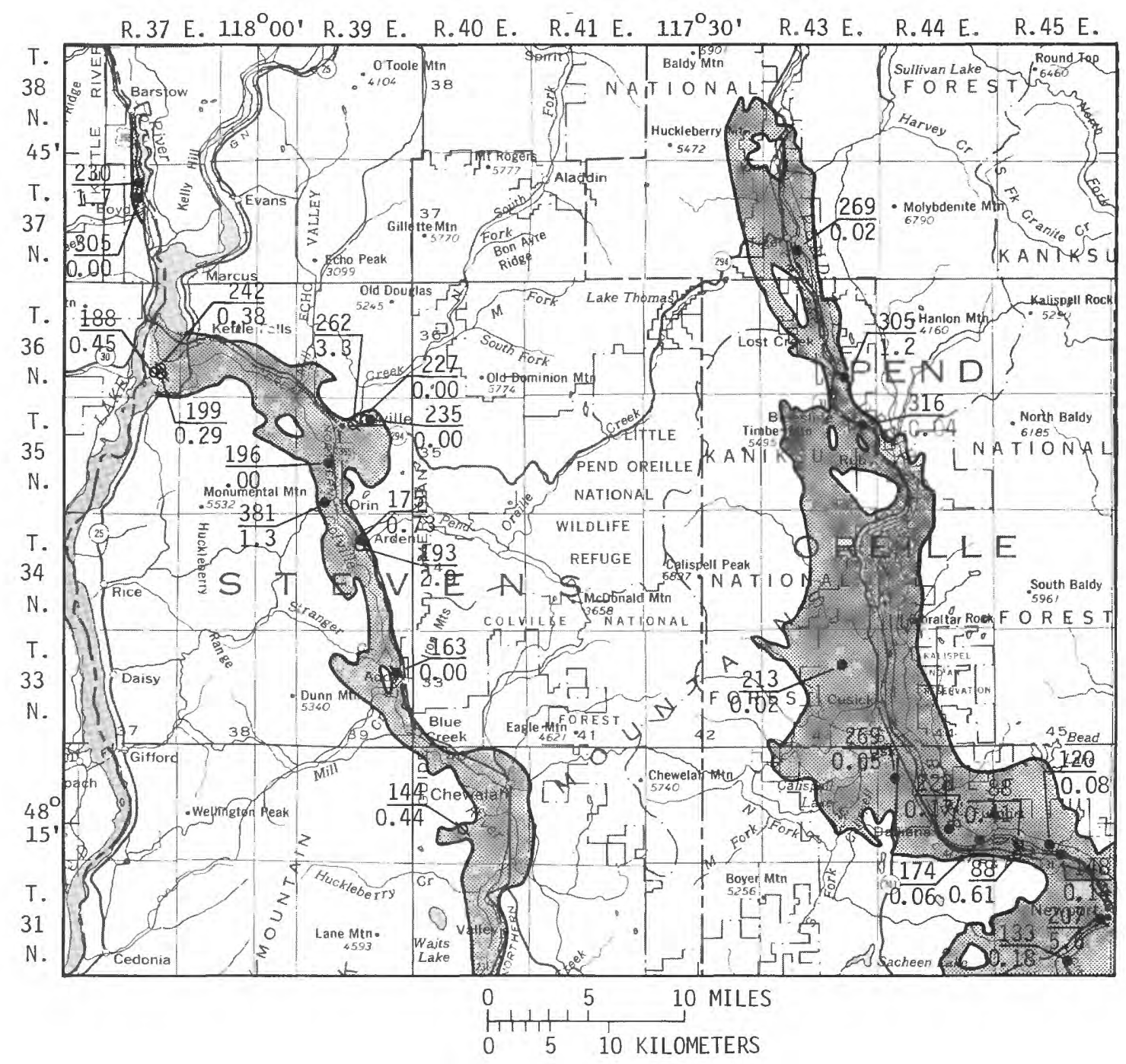

EXPLANATION

Area underlain by unconsolidated alluvial and glacial deposits in the vicinity of the sample sites. Source: Huntting, M.T., and others, 1961, Geologic Map of Washington.
- Well sampled in 1979

- Well sampled prior to 1979

Q Well sampled for trace metals $\frac{144 \text { dissolved solids, in } \mathrm{mg} / \mathrm{L}}{0.44 \text { nitrate as } \mathrm{N} \text {, in } \mathrm{mg} / \mathrm{L}}$

FIGURE 14.--The Colville-Kettle and Pend Oreille subregions showing sample sites and average nitrate and dissolved-solids concentrations. 


\section{EXPLANATION}

- Samples collected in 1979

- Samples collected prior to 1979

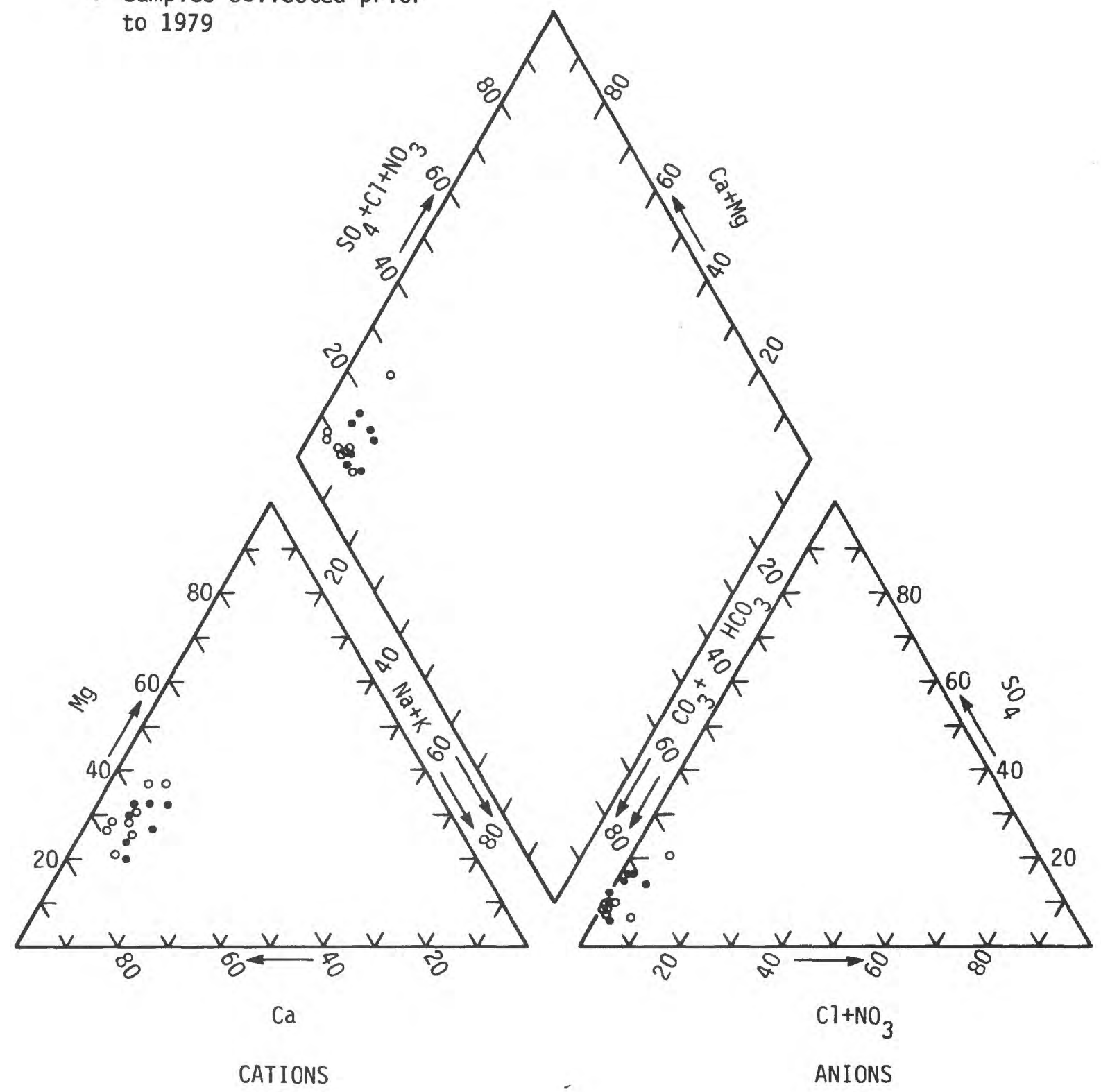

FIGURE 15.--Major ion percentages in water from the Colville-Kettle subregion wells. 


\section{Pend Oreille Subregion}

Alluvial and glacial deposits along the Pend Oreille River form the principal aquifers in the Pend Oreille subregion. The wells sampled in the subregion extend from the vicinity of Newport north to Tiger (fig. 14). Ground water in the subregion is used primarily for domestic, municipal, and irrigation purposes.

Ground-water-quality data for the subregion appear in tables 22 and 24 and are summarized in table 23. In 5 of the 12 samples for which a manganese determination was done, concentrations exceeded the recommended limit of 50 $\mathrm{ug} / \mathrm{L}$. Iron concentrations exceeded the recommended limit of $300 \mathrm{ug} / \mathrm{L}$ in 3 of 13 samples. No other constituents exceeded maximum contaminant levels specified by U.S. Environmental Protection Agency primary or secondary drinking water regulations.

Nitrate concentrations were generally low. The median $\mathrm{NO}_{3}-\mathrm{N}$ concentration was $0.14 \mathrm{mg} / \mathrm{L}$. The sample from well $31 / 45-24 \mathrm{Bl}$ had a nitrate concentration of $5.6 \mathrm{mg} / \mathrm{L}$. The source of nitrate is unknown, but on the basis of other data it appears to be local. As shown in figure 16, the major ions in the ground water sampled were calcium, magnesium, and bicarbonate. Hardness values ranged from soft to hard. 


\section{EXPLANATION}

- Samples collected in 1979

- Samples collected prior

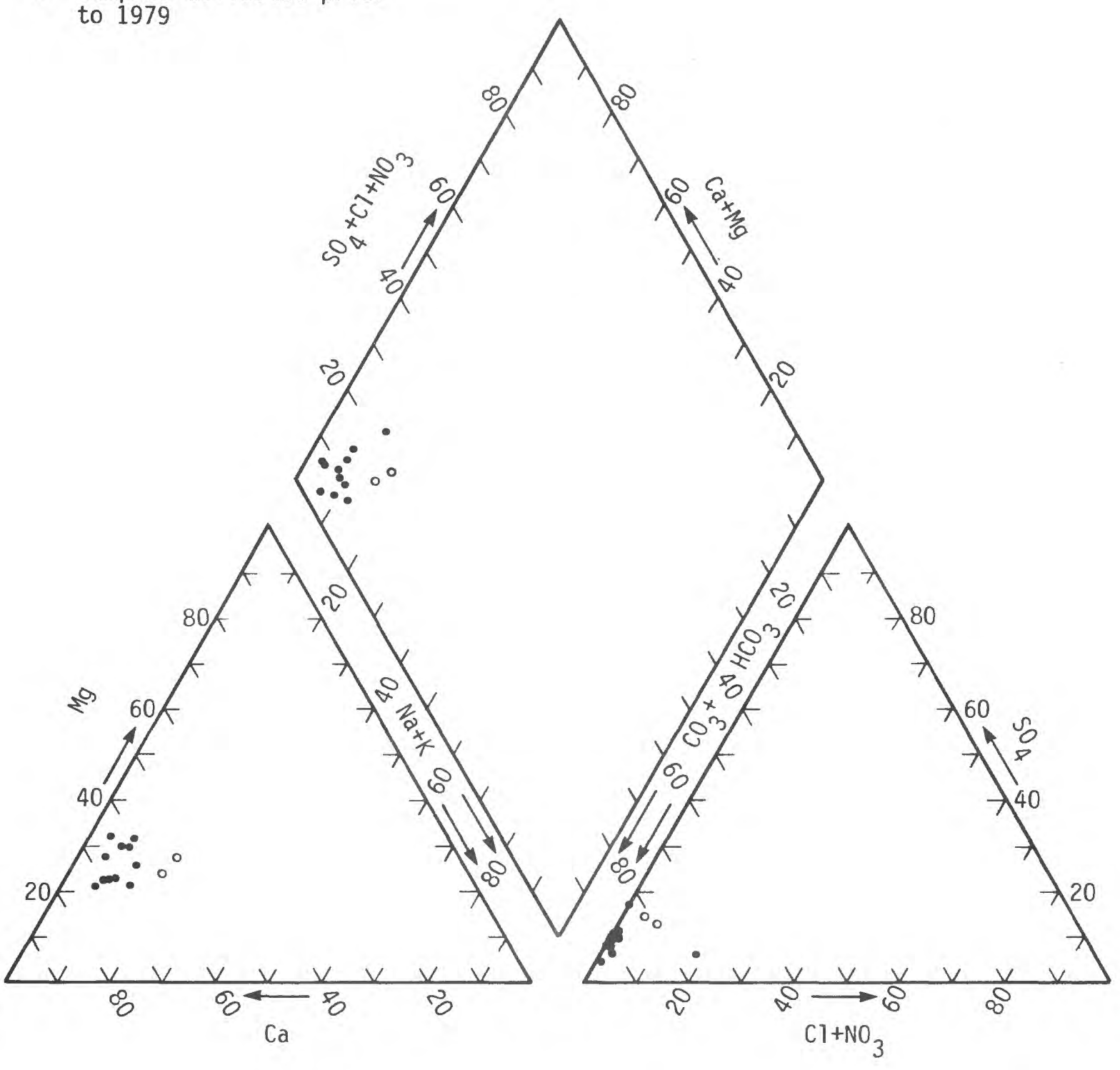

CATIONS

ANIONS

FIGURE 16.--Major ion percentages in water from the Pend Oreille subregion wells. 


\section{OKANOGAN REGION}

The Okanogan Region is located in north-central Washington, and its boundaries enclose the part of the Okanogan River basin that lies within the United States (fig. 1). Alluvial and glacial deposits, which occur primarily in and adjacent to the major valleys, contain the main volume of ground water in the region (fig. 17). Ground water withdrawn from the aquifer is used for agricultural, industrial, municipal, and domestic purposes.

Physical, chemical, and biological water-quality data for wells sampled in the Okanogan Region are shown in table 25 and are summarized in table 26. Additional trace-element data appear in table 27.

The median values for dissolved-solids concentrations were $359 \mathrm{mg} / \mathrm{L}$ for analytical determinations (residue or evaporation at $180^{\circ} \mathrm{C}$ ) and $340 \mathrm{mg} / \mathrm{L}$ based on the sum of dissolved constituents. The recommended limit for the dissolved-solids concentration in drinking water of $500 \mathrm{mg} / \mathrm{L}$ was exceeded in 5 of 28 samples. For irrigation purposes the salinity hazard of ground-water samples ranged from low to high, and the sodium hazard was low in all samples except from well $38 / 27 \mathrm{E}-10 \mathrm{~N} 1$, where it was high.

Figure 18 illustrates the variation in the chemical composition of ground water sampled in the region. There is a distinct variability in anionic composition where, in many samples, percentages of carbonate and bicarbonate are low compared with ground water from other northeastern-north central aquifer regions. There was less dispersion in the plot of cation percentages than anion percentages; however, the high percentage of sodium in water from well $38 / 27 \mathrm{E}-10 \mathrm{Nl}$ is unique compared with other samples.

In addition to dissolved solids, other constituent concentrations found to exceed maximum contaminant levels specified by U.S. Environmental Protection Agency primary and secondary drinking water regulations were iron, manganese, sulfate, and selenium. Iron concentrations in excess of the recommended limit of $300 \mathrm{ug} / \mathrm{L}$ were found in samples from four wells; however, excessive iron in ground water does not appear to be a pervasive problem in the region. Median concentrations for dissolved and total-recoverable iron were 10 and $50 \mathrm{ug} / \mathrm{L}$, respectively. Manganese concentrations in samples from 5 of 17 wells exceeded the recommended limit of $50 \mathrm{ug} / \mathrm{L}$. The five wells were scattered throughout the region, indicating local influences rather than extensive areas where the ground water had high manganese concentrations. Two samples contained dissolved sulfate in excess of the recommended limit of $250 \mathrm{mg} / \mathrm{L}$. Although a graph showing the percentage composition of anions indicates some shift toward sulfate and chloride (fig. 18), the median sulfate concentration of $73.5 \mathrm{mg} / \mathrm{L}$ is well below the recommended limit.

The only constituent to exceed maximum contaminant limits set by the U.S. Environmental Protection Agency primary drinking water regulations was selenium, at a concentration of $12 \mathrm{ug} / \mathrm{L}$ in water from irrigation well $30 / 25 \mathrm{E}-10 \mathrm{~N} 1$. 


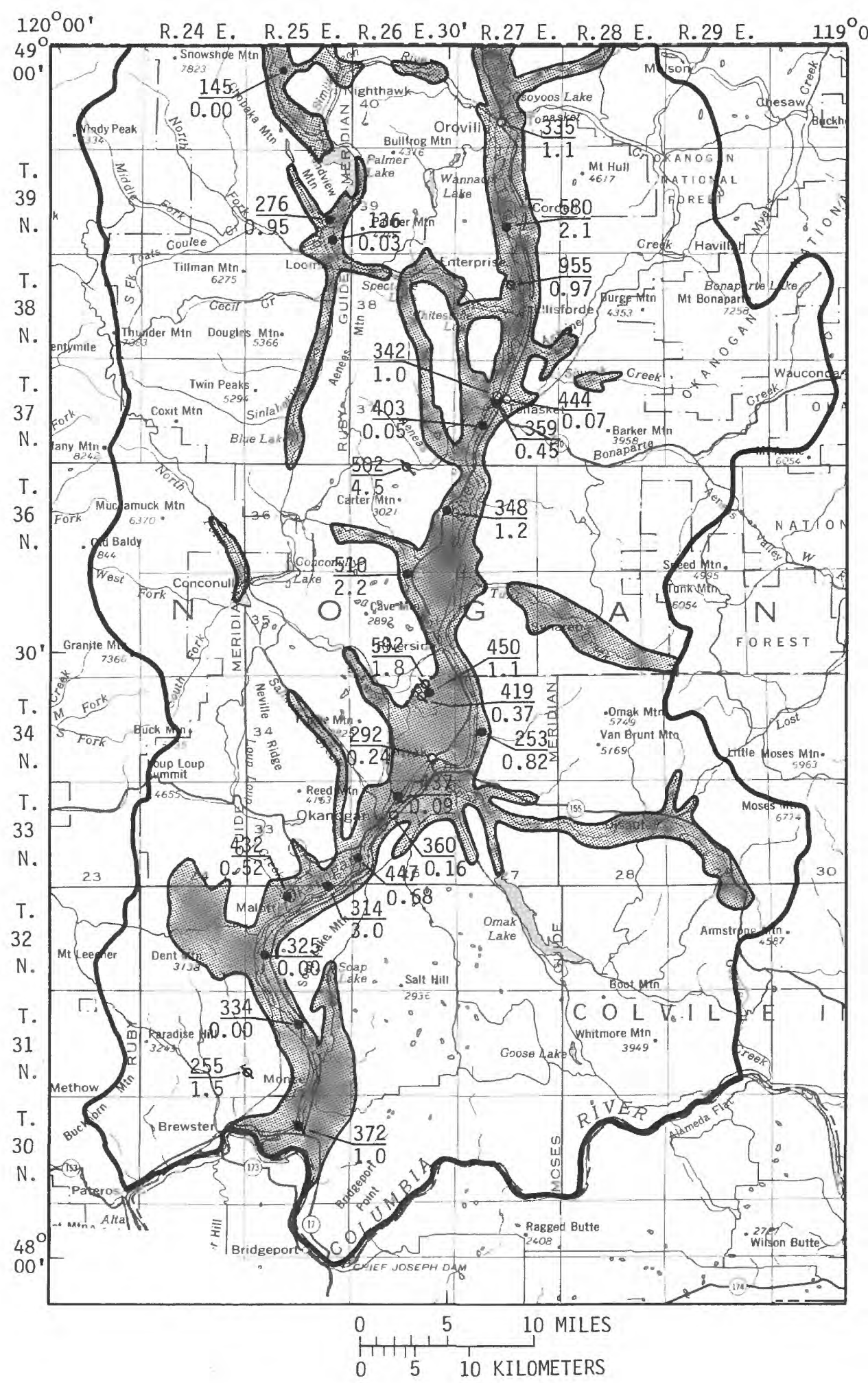

EXPLANATION

Area underiain by unconsolidated alTuvial or glacial deposits of sufficient thickness to provide yieids of water to wells.

Source: Walters (1974).
- Well sampled in 1979

- Well sampled prior to 1979

Q Well sampled for trace metals

$\frac{447 \text { dissolved solids, in } \mathrm{mg} / \mathrm{L}}{0.68 \text { nitrate as } \mathrm{N} \text {, in } \mathrm{mg} / \mathrm{L}}$

0.68 nitrate as $\mathrm{N}$, in $\mathrm{mg} / \mathrm{L}$
Boundary of aquifer region

FIGURE 17.--The Okanogan Region showing sample sites and average nitrate and dissolved-solids concentrations. 


\title{
EXPLANATION
}

- Samples collected in 1979

- Samples collected prior to 1979

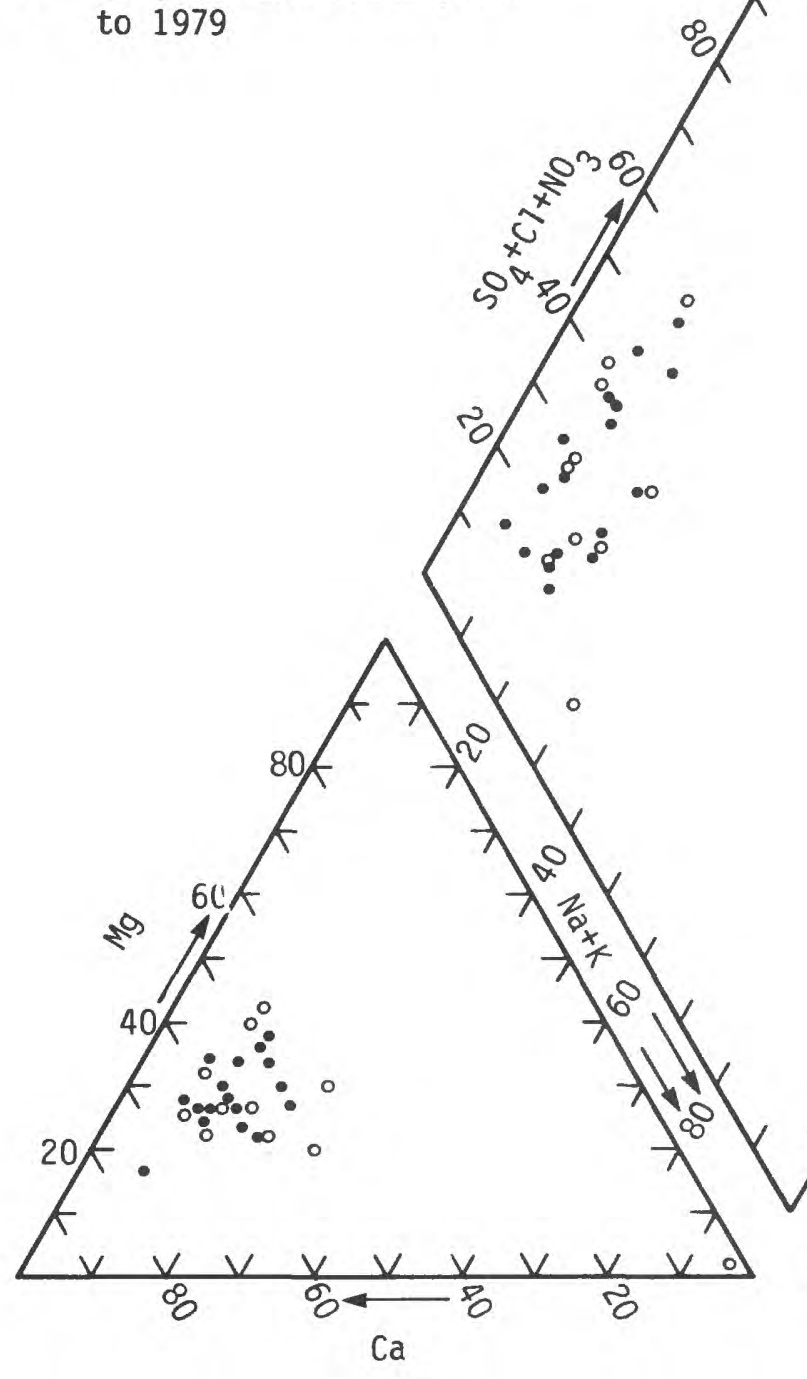

CATIONS

\author{
or
}

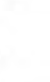




\section{METHOW REGION}

The Methow Region is located in the western one-third of Okanogan County, between the crest of the Cascade Range and the Okanogan River basin (fig. 1). Population in the region is sparse and is concentrated along the Methow River valley between Mazama and Pateros.

Alluvial and glacial deposits along the Methow River and its tributaries constitute the major ground-water reservoir in the region (fig. 19). Ground water is used for agricultural, industrial, municipal, and domestic purposes.

Wells sampled in the Methow aquifer region are shown in figure 19. Physical, chemical, and biological water-quality data for water from these wells appear in tables 28 and 30 and are summarized in table 29. These data indicate that the ground water in the region is of adequate quality for most uses. None of the maximum contaminant levels specified by U.S. Environmental Protection Agency primary and secondary drinking water regulations was exceeded. The hardness of the ground water ranged from soft to moderately hard, making the water acceptable for most uses. Calcium and magnesium were the major cations and bicarbonate was the principal anion in the samples (fig. 20). The ground water had a low sodium-adsorption ratio, indicating its suitability for irrigation. 


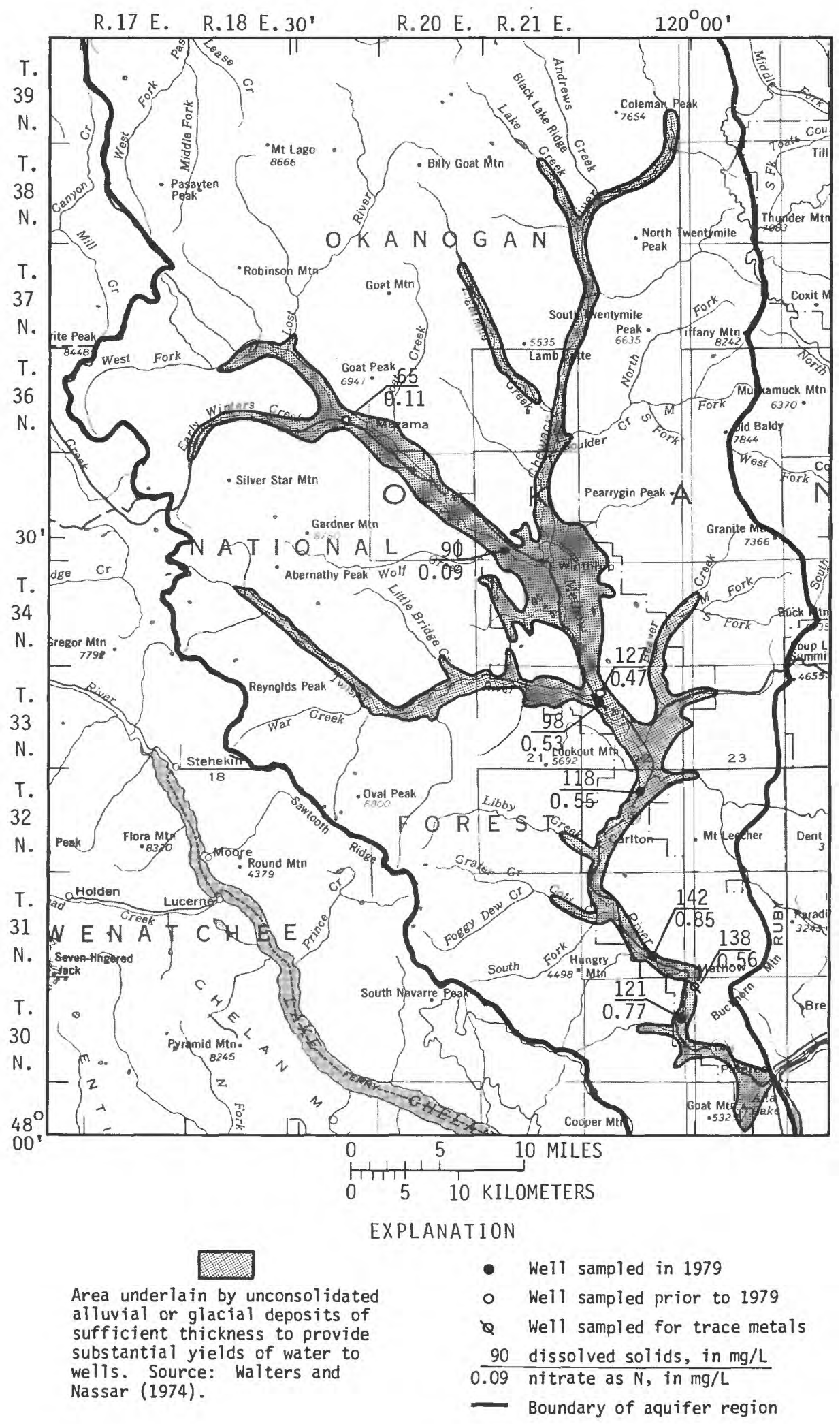

FIGURE 19.--The Methow Region showing sample sites and average nitrate and dissolved-solids concentrations. 


\title{
EXPLANATION
}

- Samples collected in 1979

- Samples collected prior to 1979

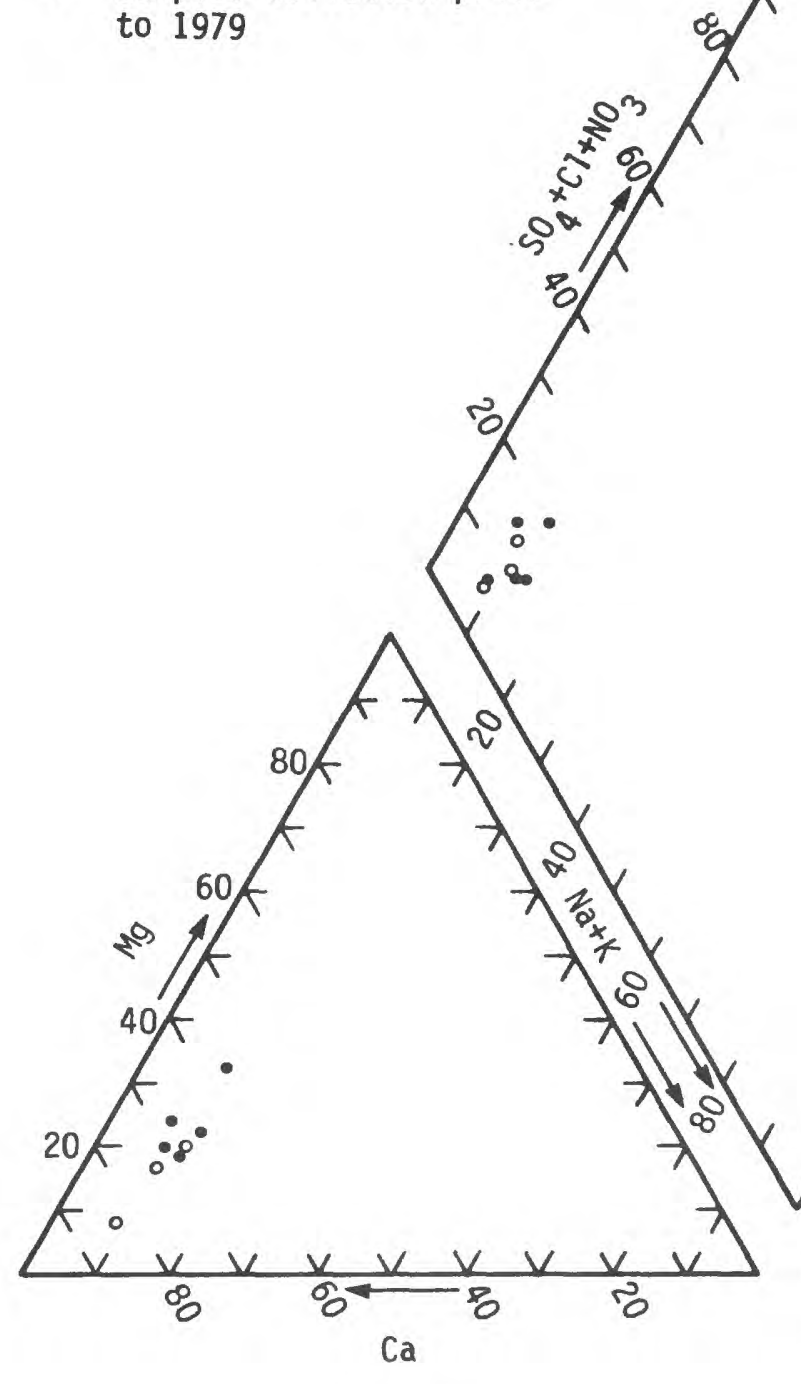

CATIONS

\author{
r
}


The Chelan Region lies between the Entiat-Wenatchee Region and the Methow Region, extending from the crest of the Cascade Range to the Columbia River (fig. 1). The principal aquifers in the region are the glacial and alluvial deposits along the Stehekin River, a tributary to Lake Chelan, and the glacial terrace deposits along the lower portion of the lake. In the vicinity of Manson, some wells have also been developed in the pre-Tertiary formations, which are chiefly metamorphic, granitic, and consolidated sedimentary rocks with low permeabilities.

The three wells sampled in the region are shown in figure 21. Ground-water development in the area is limited, and most wells are along the lower end of the lake in the terrace deposits or in the hills above the lake. Because of the limited development, only two wells, $28 / 22-32 \mathrm{Pl}$ and $28 / 22-28 \mathrm{Bl}$, were sampled during 1979. Well 28/22-21 J1 was sampled in 1971 .

Physical, chemical, and biological water-quality data for ground-water samples from the region are shown in table 31 . Figure 22 illustrates the variation in chemical composition of ground water sampled in the region. Water from well 28/22-32 Pl, which was terminated in glacial deposits, had a higher percentage of sodium than water from the wells 28/22-32Pl and 28/22-28Bl, which were terminated in the pre-Tertiary rocks. Dissolved-solids concentrations in samples from the three wells ranged from 349 to $480 \mathrm{mg} / \mathrm{L}$, and hardness ranged from moderately hard to very hard. 


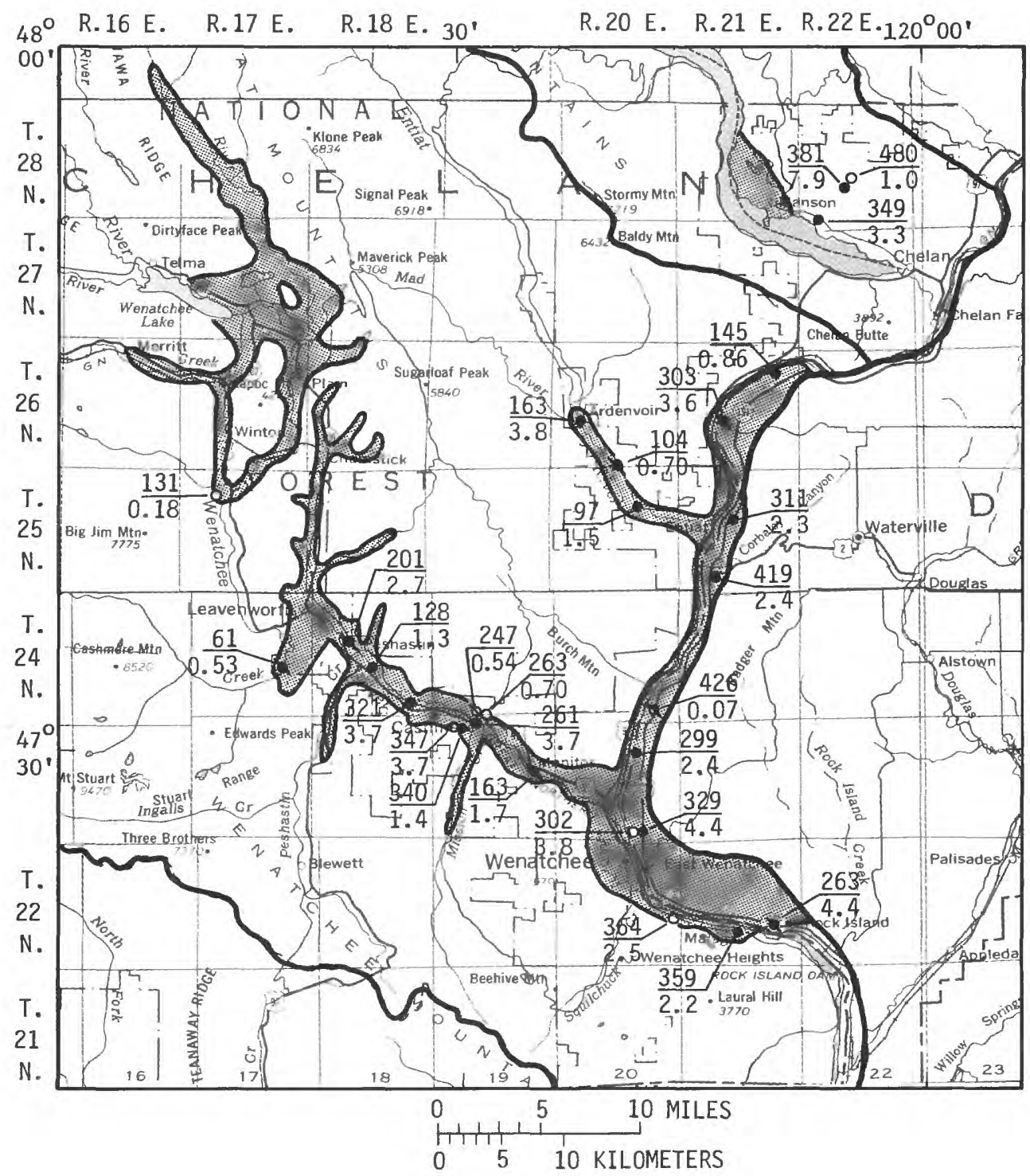

EXPLANATION

Area underlain by unconsolidated alluvial and glacial deposits in the vicinity of the sample sites. Source: Huntting, M.T., and others, 1961, Geologic Map of Washington.
- Well sampled in 1979

- Well sampled prior to 1979

Q Well sampled for trace metals

201 dissolved solids, in $\mathrm{mg} / \mathrm{L}$

2.7 nitrate as $\mathrm{N}$, in $\mathrm{mg} / \mathrm{L}$

- Boundary of aquifer region

FIGURE 21.--The Chelan and Entiat-Wenatchee Regions showing sample sites and average nitrate and dissolvedsolids concentrations. 


\title{
EXPLANATION
}

- Samples collected in 1979

- Samples collected prior to 1979

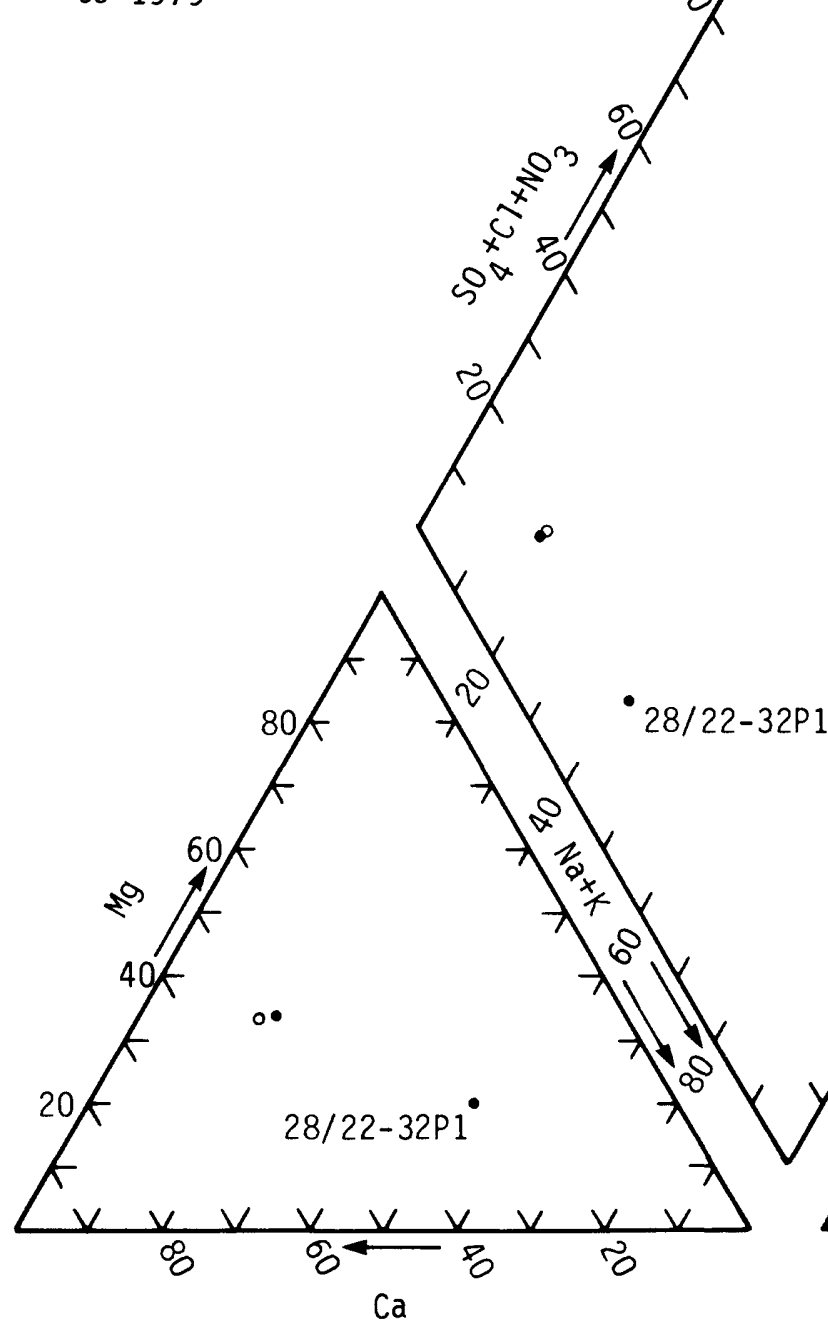

CATIONS

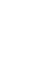




\section{ENTIAT-WENATCHEE REGION}

The principal aquifers in the Entiat-Wenatchee Region are the alluvial and glacial deposits in the Entiat and Wenatchee River valleys and the alluvial and glacial deposits along the Columbia River (fig. 21). On the east side of the Columbia River in Douglas County, wells extending from Rock Island north to township $27 \mathrm{~N}$. were sampled. In Chelan County, most sample sites were located along the Entiat and Wenatchee River valleys; however, two wells were sampled on the west side of the Columbia River near Malaga. Ground water withdrawn from these aquifers is used for agricultural, industrial, municipal, and domestic purposes.

In the following discussion on ground-water quality, the region is divided into the Entiat subregion, which includes the Entiat River valley and the adjacent area on the eastern side of the Columbia River, and the Cashmere subregion, which includes the Wenatchee River valley and the area in townships 22,23 , and $24 \mathrm{~N}$., adjacent to the Columbia River.

\section{Entiat Subregion}

In the Entiat subregion, three wells were sampled along the Entiat River valley and four were sampled along the Columbia River in Douglas County. The water-quality data for the subregion appear in tables 32 and 34 and are summarized in table 33. None of the constituent concentrations exceeded maximum contaminant levels specified by U.S. Environmental Protection Agency primary and secondary drinking water regulations. Dissolved-solids concentration samples ranged from 97 to $394 \mathrm{mg} / \mathrm{L}$. The median dissolved-solids concentration in the samples collected from wells along the Entiat River was $104 \mathrm{mg} / \mathrm{L}$, and for samples collected in Douglas County, $307 \mathrm{mg} / \mathrm{L}$. Although the median dissolved-solids concentration was higher in the Douglas County samples, there was no definitive difference in the percentage composition of cations and anions in samples from the two counties (fig. 23). Samples from well 26/21 E-21 N2 had a higher percentage of sulfate compared with other samples from the subregion.

For irrigation purposes, the ground water sampled had a low sodium hazard and a low to medium salinity hazard.

\section{Cashmere Subregion}

Wells sampled in the Cashmere subregion include those in Chelan County in the Wenatchee River valley and along the Columbia River, and those along the Columbia River in Douglas County (fig. 21).

Plots of the percentages of anions and cations in samples from the subregion appear in figure 24, and water-quality data are summarized in tables 35,36 , and 37. In all samples except from well $24 / 20 \mathrm{E}-35 \mathrm{Jl}$, bicarbonate is the major anion. There is, however, considerable variation in the percentages of calcium and magnesium. Calcium was the principal cation in samples from wells adjacent to the Columbia River in Douglas County. The only sample from a well adjacent to the Columbia River in Chelan County with a complete cation-anion analysis was from well $22 / 20 \mathrm{E}-24 \mathrm{Rl}$. Calcium was also the principal cation in that sample (fig. 24). Samples from wells in the Wenatchee River valley had, in general, a higher percentage of magnesium than samples from wells along the Columbia River. Samples with the highest percentage magnesium were from wells in township $24 \mathrm{~N}$. and ranges 17 and $18 \mathrm{E}$. 
Dissolved-solids concentrations in samples from Douglas County wells ranged from 238 to $426 \mathrm{mg} / \mathrm{L}$, and from wells in Chelan County, from 41 to $364 \mathrm{mg} / \mathrm{L}$. Well 24/17E-23Ql, which is adjacent to Icicle Creek, was sampled in 1970 and 1979. Dissolved-solids concentrations in these samples were 81 and $41 \mathrm{mg} / \mathrm{L}$, respectively, which were the lowest of the subregion.

Nitrate concentrations in ground-water samples from the subregion appear on figure 21 and in table 35 . The median $\mathrm{NO}_{3}-\mathrm{N}$ concentration was $2.7 \mathrm{mg} / \mathrm{L}$, and there were few samples in which the $\mathrm{NO}_{3}-\mathrm{N}$ concentration was less than $1 \mathrm{mg} / \mathrm{L}$. In the upper part of the Wenatchee River basin, $\mathrm{NO}_{3}-\mathrm{N}$ concentrations were less than $1 \mathrm{mg} / \mathrm{L}$ in samples from well $25 / 17 \mathrm{E}-8 \mathrm{Gl}$, sampled in 1965 , and in well 24/17E-23Q1, sampled in 1970 and 1979. $\mathrm{NO}_{3}-\mathrm{N}$ concentrations of 0.70 and 0.54 $\mathrm{mg} / \mathrm{L}$ were found in samples from City of Cashmere wells $23 / 19 \mathrm{E}-24 \mathrm{Dl}$ and 23/19E-24D2, respectively. These wells were sampled in 1961 and 1939. A third City of Cashmere well, 24/19E-24E l, located near wells $-\mathrm{D} 1$ and $-\mathrm{D} 2$ and was sampled in 1979, had a $\mathrm{NO}_{3}-\mathrm{N}$ concentration of $3.7 \mathrm{mg} / \mathrm{L}$. In Douglas County, well $24 / 20 \mathrm{E}-35 \mathrm{Jl}$ was sampled in $197 \mathrm{l}$, and the nitrate concentration of the sample was $0.07 \mathrm{mg} / \mathrm{L}$ as $\mathrm{N}$. No other water from wells in the subregion sampled during or prior to 1979 had a lower nitrate concentration; however, this well, which is terminated at $260 \mathrm{ft}$, is over $100 \mathrm{ft}$ deeper than any of the other wells sampled.

The only constituents in ground-water samples from the subregion that exceeded maximum contaminant levels specified by U.S. Environmental Protection Agency primary and secondary drinking water regulations were iron and manganese (table 35). For irrigation purposes, the ground water sampled had a low sodium hazard and a low to medium salinity hazard. 


\section{EXPLANATION}

- Chelan County

- Douglas County

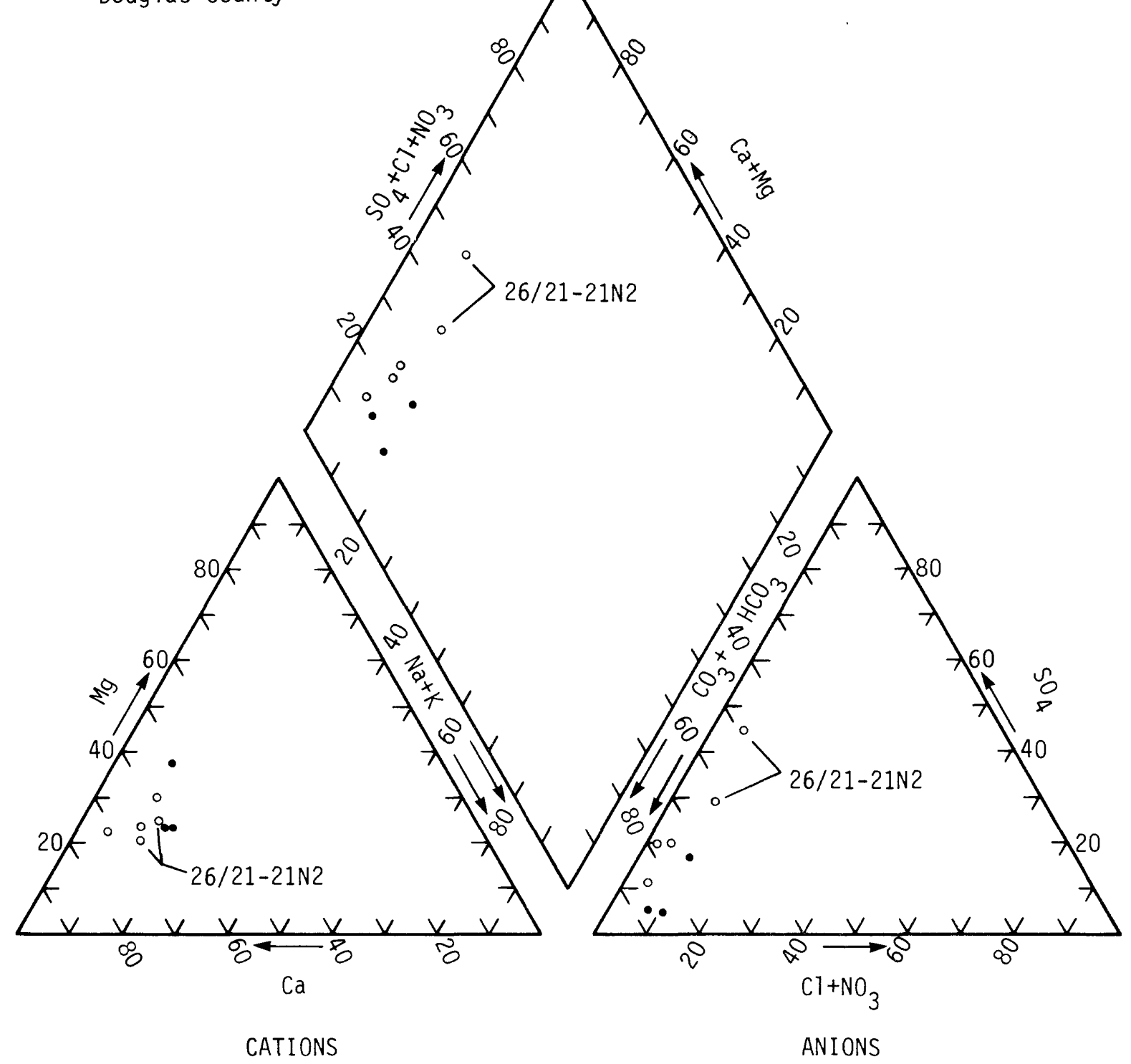

FIGURE 23.--Major ion percentages in water from the Entiat subregion wells. 


\section{EXPLANATION}

- Chelan County

- Douglas County

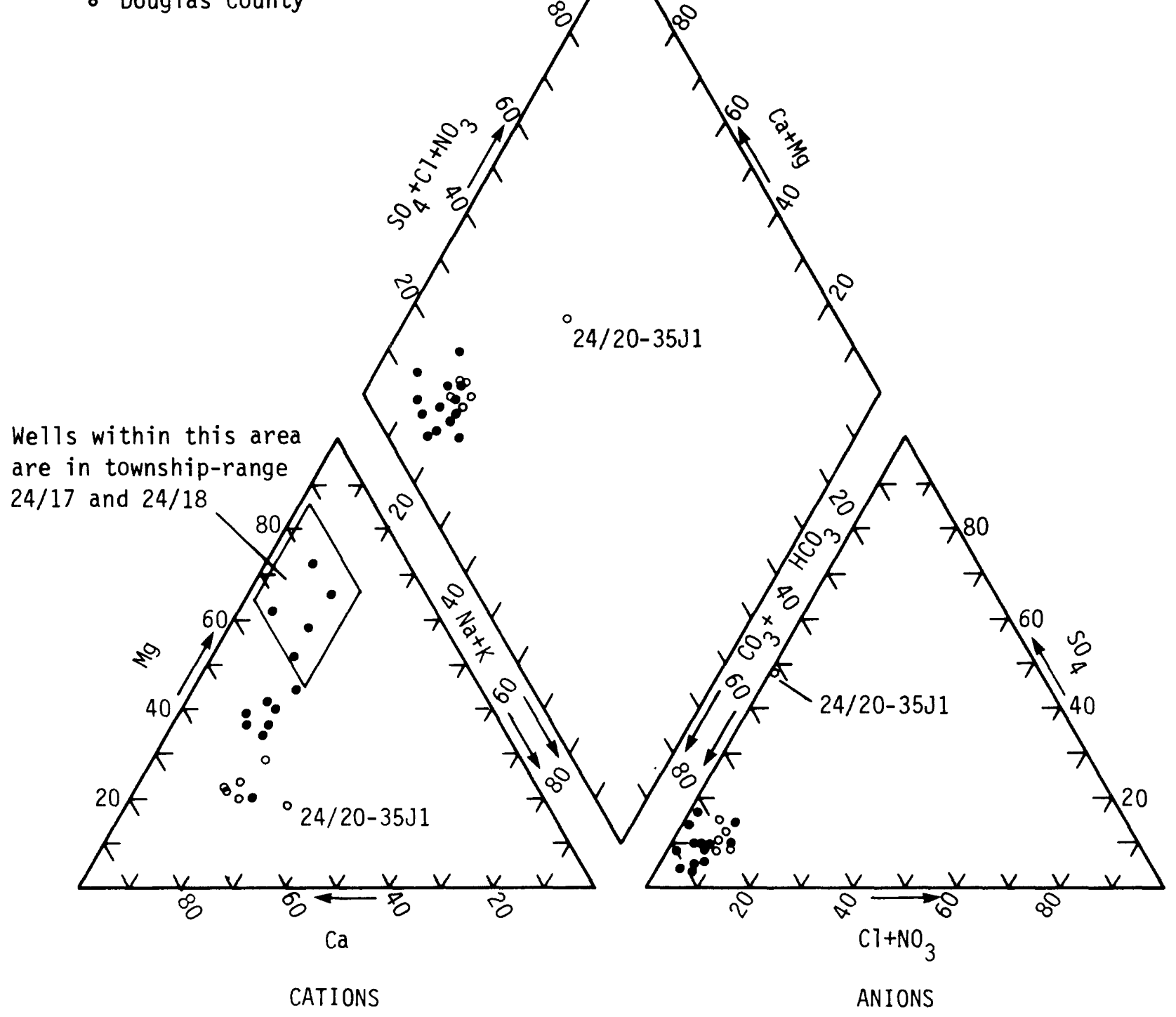

FIGURE 24.--Major ion percentages in water from the Cashmere subregion wells. 
Bolke, E. L., and Vaccaro, J. V., 1979, Selected hydrologic data for Spokane Valley, Spokane, Washington, 1977-78: U.S. Geological Survey Open-File Report 79-333, 98 p.

Dion, N. P., and Lum, W. E., II, 1977, Municipal, industrial, and irrigation water use in Washington, 1975: U.S. Geological Survey Open-File Report 77-308, $34 \mathrm{p}$.

Drost, B. W., and Seltz, H. R., 1978, Spokane Valley-Rathdrum Prairie Aquifer, Washington and Idaho: U.S. Geological Survey Open-File Report 77-829, 79 p.

Faust, S. D., and Mikulewicz, E.W., 1967, Factors influencing the condensation of 4-aminoantipyrine with derivatives of hydroxybenzene-II. influence of hydronium ion concentration on absorptivity: Water Research, v. 1, p. 509-522.

Harkness, R. E., Myers, D. A., and Bortleson, G.C., 1974, Water resources of the Colville Indian Reservation, Washington: U.S. Geological Survey Open-File Report, 149 p.

Hem, J.D., 1970, Study and interpretation of the chemical characteristics of natural water ( $2 \mathrm{~d}$ ed.): U.S. Geological Survey Water-Supply Paper $1473,363 \mathrm{p}$.

Huntting, M.T., Bennett, W.A.G., Livingston, V. E., Jr., and Moen, V. S., 1961, Geologic map of Washington: Washington Division of Mines and Geology, 1:500,000, two sheets.

Luzier, J. E., and Burt, R. J., 1974, Hydrology of basalt aquifers and depletion of ground water in east-central Washington: Washington Department of Ecology Water-Supp1y Bulletin 33, 53 p.

Molenaar, Dee, Grimstad, P., and Walters, K. L., 1980, Principal aquifers and well yields in Washington: U.S. Geological Survey Geohydrologic Monograph 5, 1:500,000, one sheet.

National Academy of Sciences, National Academy of Engineers, 1974, Water quality criteria: EPA-R3-73-033, $594 \mathrm{p}$.

01son, T. M., and others, 1975, Geology, ground water, and water quality of part of southern Spokane County, Washington: Eastern Washington State College, 139 p.

Sawyer, C. N., 1960, Chemistry for sanitary engineers: New York, McGrawHi11, 367 p.

U.S. Department of Agriculture, 1954, Diagnosis and improvement of saline and alkall solls: U.S. Department of Agriculture Handbook 60, 156 p. 
U.S. Environmental Protection Agency, 1976, National interim primary drinking water regulations: U.S. Government Printing office, 159 p.

- - 1977a, National secondary drinking water regulations: Proposed regulations: Federal Register, v. 42, no. 62, p. 17143-17146.

---1977b, Quality criteria for water, 1976: Washington D. C., 256 p.

----1980, Water quality criteria documents; availability: Federal Register, v. 45, no. 231, p. 79318-79382.

Vaccaro, J.J., and Bolke, E.L., 1983, Evaluation of water quality characteristics of part of the Spokane aquifer, Washington and Idaho, using a solute-transport digital model: U.S. Geological Survey Water-Resources Open-File Report 82-769, 69 p.

Viets, F.G., Jr., and Hageman, R. H., 1971, Factors affecting the accumulation of nitrate in soil, water, and plants: U.S. Department of Agriculture, Handbook No. 413, 63 p.

Walters, K. L., 1974, Water in the Okanogan River basin, Washington: Washington Department of Ecology Water-Supply Bulletin 34, 136 p.

Walters, K. L., and Nassar, E. G., 1974, Water in the Methow River basin, Washington: Washington Department of Ecology Water-Supply Bulletin $38,73 \mathrm{p}$. 


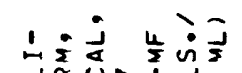

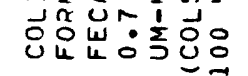

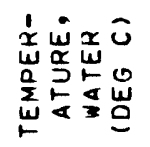$$
\frac{\text { बे }}{\frac{\pi}{2}}
$$

$$
\text { 竞芯 }
$$

, 는ํํำ

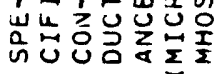
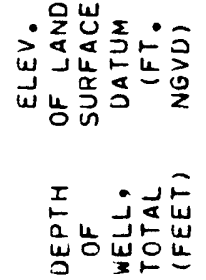

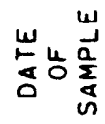

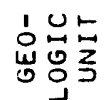

范

这方,

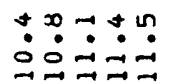

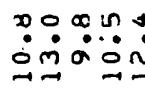

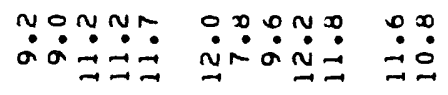

ำํ⿻上丨丶

เกา แก แก ?

$\because \div \div \because 0$

$\because \infty \infty a ?$

$\infty \div$

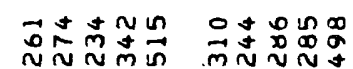

$\vec{m} \vec{m} \tilde{0} \cdot \overrightarrow{0}$

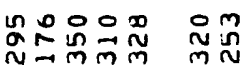

응응용요

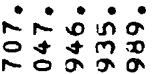

웅으요.

ภำ次告

응응응응 응응응

iि $\dot{0} \dot{0} \dot{0}$

กิบี๋ำ

$\dot{0} \dot{0} \dot{\sim} \dot{0} \dot{0}$

용

운줌

กNN=n

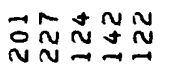

은ํํํํํ요

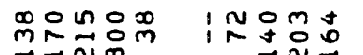

$\underset{\substack{\infty \\ \sim}}{\substack{c \\ \sim}}$

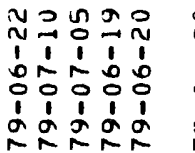

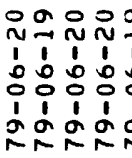

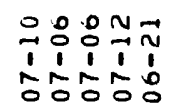

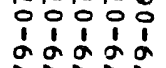

$\vec{\sim} \vec{N} \vec{N}_{\text {N }} \vec{N}$

$\begin{array}{llllll}1 & 1 & 1 & 1 & 1 & 1 \\ 0 & 0 & 0 & 0 & 0 & 0 \\ 0 & 1 & 1 & 0 & 0\end{array}$

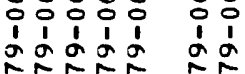

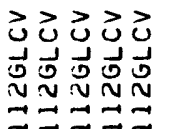

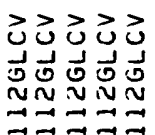

记导文

ज़

$\Xi \Xi \Xi \Xi$

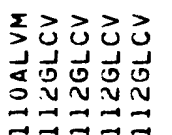

岂导
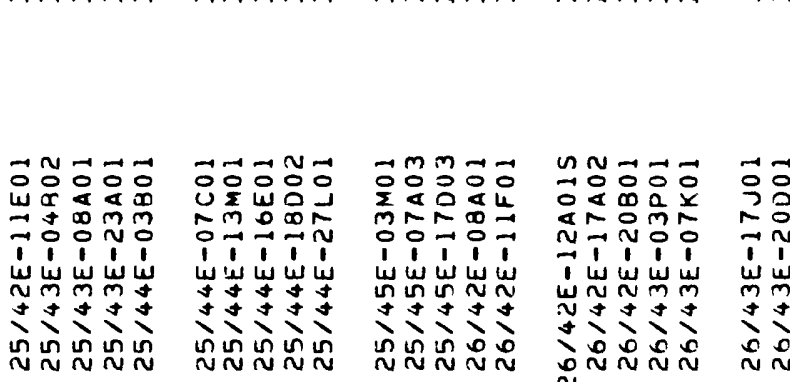

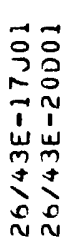


ذُّ

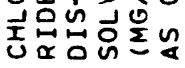

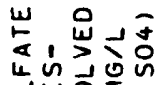
可出的经的

$12+\hat{m}$

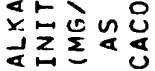
, wำ 造造造

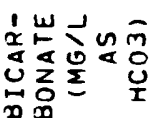

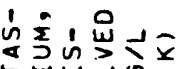

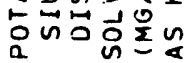

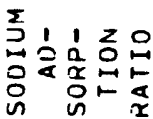

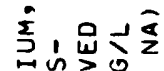
응무인

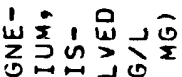

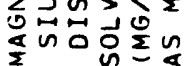

至, 虫

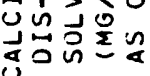

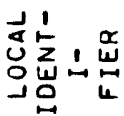

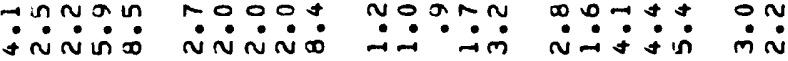

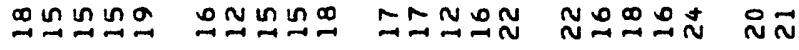

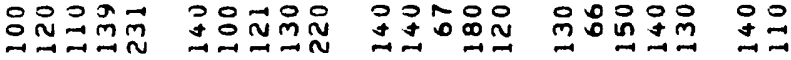

0000000000000000000000

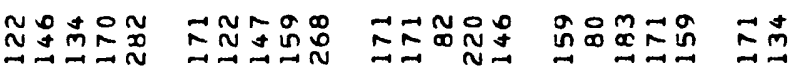

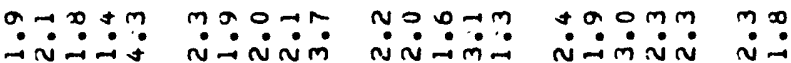

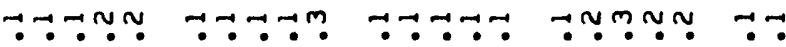

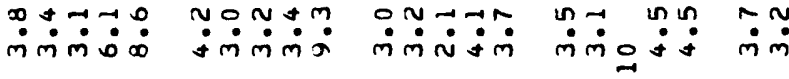

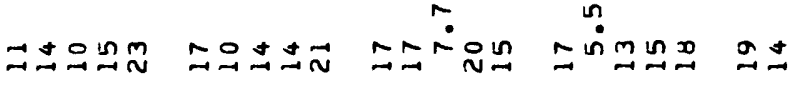

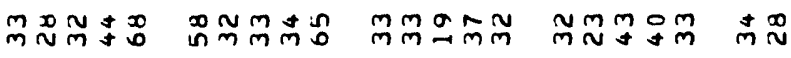
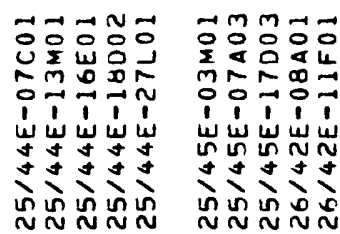

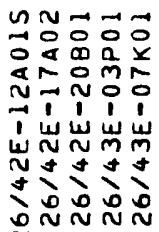

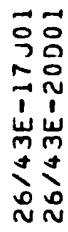


造的岀芳

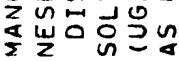

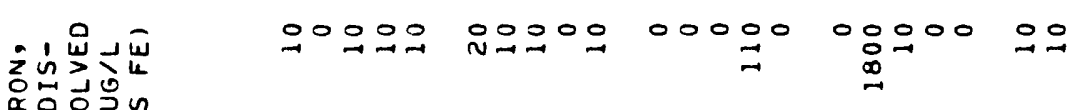

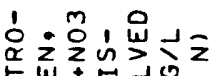

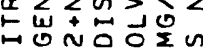



证的的品

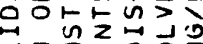

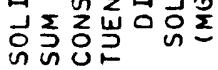

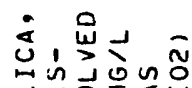

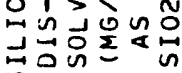

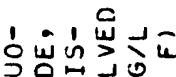

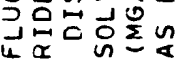

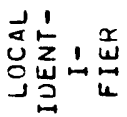

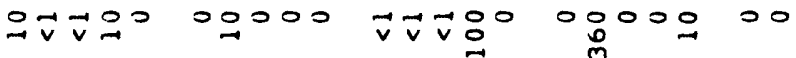

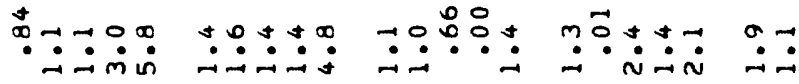

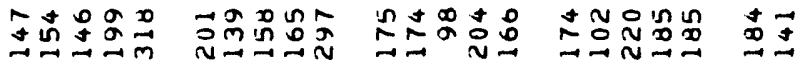

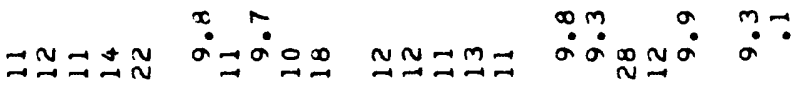

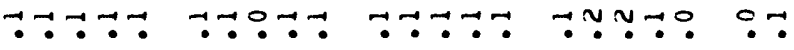

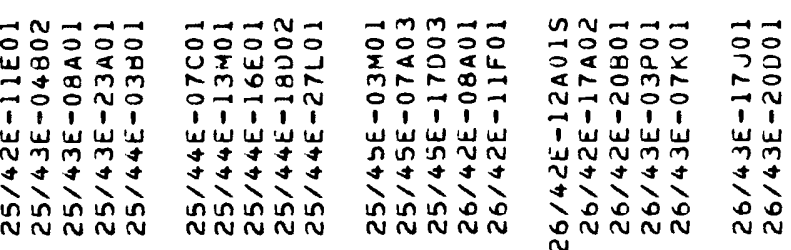


TABLE 4,--Summary of physical, biological, and major chemical-constituent data for Spokane Valley ground water sampled during 1979

[Values in milligrams per liter except as indicated umho, micromho; col/100 mL, colonies per 100 milliliter; ug/L, microgram per liter]

\begin{tabular}{|c|c|c|c|c|}
\hline Constituent & $\begin{array}{l}\text { Number of } \\
\text { sample sites }\end{array}$ & $\frac{\text { Trace eler }}{\text { Maximum }}$ & $\frac{\text { ent concel }}{\text { Minimum }}$ & $\frac{\text { tration }}{\text { Median }}$ \\
\hline Specific conductance (umho) & 22 & 515 & 167 & 303 \\
\hline $\mathrm{pH}$ (units) & 22 & 8.2 & 7.4 & 7.6 \\
\hline Temperature $\left({ }^{\circ} \mathrm{C}\right)$ & 22 & 7.4 & 8.2 & 7.75 \\
\hline Fecal-coliform bacteria $(c 01 / 100 \mathrm{~mL})$ & 21 & 14 & $<1$ & $<1$ \\
\hline Hardness (as $\mathrm{CaCO}_{3}$ ) & 22 & 260 & 79 & 150 \\
\hline Hardness, noncarbonate (as $\mathrm{CaCO}_{3}$ ) & 22 & 70 & 0 & 20 \\
\hline Calcium, dissolved & 22 & 68 & 19 & 33 \\
\hline Magnesium, dissolved & 22 & 23 & 5.5 & 15 \\
\hline Sodium, dissolved & 22 & 10 & 2.1 & 3.6 \\
\hline Sodium-adsorption ratio & 22 & .3 & .1 & .1 \\
\hline Potassium, dissolved & 22 & 4.3 & 1.3 & 2.1 \\
\hline Bicarbonate & 22 & 282 & 80 & 164 \\
\hline Carbonate & 22 & 0 & 0 & 0 \\
\hline Alkalinity (as $\mathrm{CaCO}_{3}$ ) & 22 & 231 & 66 & 130 \\
\hline Sulfate, dissolved & 22 & 24 & 12 & 16.5 \\
\hline Chloride, dissolved & 22 & 8.5 & .9 & 2.6 \\
\hline Fluoride, dissolved & 22 & .2 & .1 & .1 \\
\hline Silica, dissolved (as $\mathrm{SiO}_{2}$ ) & 22 & 28 & .1 & 11 \\
\hline Solids, dissolved (residue at $180^{\circ} \mathrm{C}$ ) & -- & -- & -- & -- \\
\hline Soilds, dissolved (sum of constituents) & 22 & 318 & 98 & 174 \\
\hline Nitrate (as N) & 22 & 5.8 & .01 & 1.4 \\
\hline Iron, total recoverable $(\mathrm{ug} / \mathrm{L})$ & -- & -- & -- & -- \\
\hline Iron, dissolved $(u g / L)$ & 22 & 1,800 & 10 & 10 \\
\hline Manganese, total recoverable (ug/L) & -- & -- & -- & -- \\
\hline Manganese, dissolved (ug/L) & 22 & 360 & $<1$ & 10 \\
\hline
\end{tabular}


TABLE 5.--Summary of specific-conductance values in ground-water samples from the Spokane Valley

\begin{tabular}{|c|c|c|c|c|c|c|c|}
\hline $\begin{array}{l}\text { LOCAL } \\
\text { IDENTIF IER }\end{array}$ & $\begin{array}{l}\text { DEPTH } \\
\text { OF } \\
\text { WELL }\end{array}$ & $\begin{array}{l}\text { BEGIN } \\
\text { YEAR }\end{array}$ & $\begin{array}{l}\text { END } \\
\text { YEAR }\end{array}$ & $\begin{array}{l}\text { MAXIMUM } \\
\text { (MICRO- } \\
\text { MHOS) }\end{array}$ & $\begin{array}{l}\text { MINIMUM } \\
\text { (MICRO- } \\
\text { MHOS) }\end{array}$ & $\begin{array}{l}\text { MEAN } \\
\text { MICRO- } \\
\text { MHOSI }\end{array}$ & $\begin{array}{l}\text { NUMUER } \\
\text { OF } \\
\text { SAMPLES }\end{array}$ \\
\hline $\begin{array}{l}25 / 42 E-03 H 01 \\
25 / 42 E-11 E 01 \\
25 / 42 E-11 E 02 \\
25 / 42 E-11 M 02 \\
25 / 42 E-13801 \\
25 / 42 E-14 J 01\end{array}$ & $\begin{array}{r}124 \\
201 \\
52 \\
230 \\
200 \\
160\end{array}$ & $\begin{array}{l}1977 \\
1947 \\
1948 \\
1955 \\
1942 \\
1977\end{array}$ & $\begin{array}{l}1978 \\
1979 \\
1961 \\
1965 \\
1978 \\
1977\end{array}$ & $\begin{array}{l}300 \\
325 \\
275 \\
279 \\
410 \\
189\end{array}$ & $\begin{array}{l}251 \\
194 \\
184 \\
257 \\
288 \\
189\end{array}$ & $\begin{array}{l}273 \\
249 \\
207 \\
273 \\
344 \\
189\end{array}$ & $\begin{array}{r}3 \\
17 \\
14 \\
8 \\
10 \\
1\end{array}$ \\
\hline $\begin{array}{l}25 / 42 E-23801 \\
25 / 43 E-04 B 02 \\
25 / 43 E-08 A 01 \\
25 / 43 E-08001 \\
25 / 43 E-09601\end{array}$ & $\begin{array}{r}59 \\
227 \\
124 \\
- \\
32\end{array}$ & $\begin{array}{l}1977 \\
1952 \\
1978 \\
1942 \\
1951\end{array}$ & $\begin{array}{l}1978 \\
1980 \\
1980 \\
1942 \\
1951\end{array}$ & $\begin{array}{r}300 \\
372 \\
252 \\
245 \\
73\end{array}$ & $\begin{array}{r}258 \\
274 \\
233 \\
245 \\
73\end{array}$ & $\begin{array}{r}278 \\
326 \\
240 \\
245 \\
73\end{array}$ & $\begin{array}{l}3 \\
3 \\
3 \\
1 \\
1\end{array}$ \\
\hline $\begin{array}{l}25 / 43 E-09603 \\
25 / 43 E-10 P 02 \\
25 / 43 E-11 B 03 \\
25 / 43 E-11002 \\
25 / 43 E-11 E 02\end{array}$ & $\begin{array}{r}73 \\
96 \\
128 \\
80 \\
-\end{array}$ & $\begin{array}{l}1977 \\
1977 \\
1977 \\
1977 \\
1977\end{array}$ & $\begin{array}{l}1978 \\
1978 \\
1978 \\
1978 \\
1977\end{array}$ & $\begin{array}{l}190 \\
350 \\
145 \\
165 \\
160\end{array}$ & $\begin{array}{l}97 \\
260 \\
111 \\
145 \\
160\end{array}$ & $\begin{array}{l}149 \\
285 \\
132 \\
154 \\
160\end{array}$ & $\begin{array}{r}3 \\
15 \\
3 \\
3 \\
1\end{array}$ \\
\hline $\begin{array}{l}25 / 43 E-11 G 04 \\
25 / 43 E-11 J 03 \\
25 / 43 E-11 M 03 \\
25 / 43 E-11 N 01 \\
25 / 43 E-11 R 01\end{array}$ & $\begin{array}{r}44 \\
125 \\
98 \\
70 \\
104\end{array}$ & $\begin{array}{l}1962 \\
1960 \\
1977 \\
1977 \\
1977\end{array}$ & $\begin{array}{l}1962 \\
1962 \\
1978 \\
1978 \\
1978\end{array}$ & $\begin{array}{l}294 \\
301 \\
280 \\
320 \\
440\end{array}$ & $\begin{array}{l}294 \\
287 \\
245 \\
300 \\
281\end{array}$ & $\begin{array}{l}294 \\
293 \\
267 \\
309 \\
350\end{array}$ & $\begin{array}{r}1 \\
3 \\
16 \\
3 \\
3\end{array}$ \\
\hline $\begin{array}{l}25 / 43 E-12 H 01 \\
25 / 43 E-12 L 02 \\
25 / 43 E-13 A 01 \\
25 / 43 E-13 H 01 \\
25 / 43 E-13 R 01\end{array}$ & $\begin{array}{r}94 \\
100 \\
110 \\
71 \\
140\end{array}$ & $\begin{array}{l}1971 \\
1977 \\
1973 \\
1974 \\
1977\end{array}$ & $\begin{array}{l}1978 \\
1978 \\
1978 \\
1974 \\
1977\end{array}$ & $\begin{array}{l}360 \\
490 \\
270 \\
265 \\
275\end{array}$ & $\begin{array}{l}264 \\
340 \\
216 \\
265 \\
275\end{array}$ & $\begin{array}{l}313 \\
415 \\
257 \\
265 \\
275\end{array}$ & $\begin{array}{r}18 \\
2 \\
9 \\
1 \\
1\end{array}$ \\
\hline $\begin{array}{l}25 / 43 E-14 E 01 \\
25 / 43 E-14 K 01 \\
25 / 43 E-14 L 01 \\
25 / 43 E-15602 \\
25 / 43 E-16 K 01\end{array}$ & $\begin{array}{r}211 \\
83 \\
120 \\
129 \\
65\end{array}$ & $\begin{array}{l}1977 \\
1973 \\
1977 \\
1977 \\
1977\end{array}$ & $\begin{array}{l}1978 \\
1978 \\
1978 \\
1978 \\
1978\end{array}$ & $\begin{array}{l}255 \\
400 \\
265 \\
270 \\
290\end{array}$ & $\begin{array}{l}225 \\
225 \\
225 \\
235 \\
232\end{array}$ & $\begin{array}{l}238 \\
265 \\
244 \\
256 \\
271\end{array}$ & $\begin{array}{r}16 \\
9 \\
14 \\
3 \\
3\end{array}$ \\
\hline $\begin{array}{l}25 / 43 E-17 R O 1 \\
25 / 43 E-21 B O 1 \\
25 / 43 E-22 F 01 \\
25 / 43 E-23 A 01 \\
25 / 43 E-23 A 02\end{array}$ & $\begin{array}{r}100 \\
87 \\
77 \\
142 \\
150\end{array}$ & $\begin{array}{l}1977 \\
1977 \\
1977 \\
1970 \\
1972\end{array}$ & $\begin{array}{l}1978 \\
1977 \\
1978 \\
1979 \\
1973\end{array}$ & $\begin{array}{l}450 \\
305 \\
347 \\
370 \\
400\end{array}$ & $\begin{array}{l}380 \\
305 \\
350 \\
234 \\
280\end{array}$ & $\begin{array}{l}423 \\
305 \\
370 \\
309 \\
312\end{array}$ & $\begin{array}{r}3 \\
1 \\
3 \\
20 \\
15\end{array}$ \\
\hline $\begin{array}{l}25 / 43 E-24 G 01 \\
25 / 44 E-01 J 01 \\
25 / 44 E-01201 \\
25 / 44 E-02801 \\
25 / 44 E-02001\end{array}$ & $\begin{array}{l}144 \\
160 \\
150 \\
127 \\
129\end{array}$ & $\begin{array}{l}1971 \\
1973 \\
1977 \\
1955 \\
1973\end{array}$ & $\begin{array}{l}1973 \\
1974 \\
1978 \\
1978 \\
1979\end{array}$ & $\begin{array}{l}420 \\
325 \\
300 \\
430 \\
775\end{array}$ & $\begin{array}{l}360 \\
284 \\
280 \\
319 \\
310\end{array}$ & $\begin{array}{l}391 \\
300 \\
292 \\
352 \\
550\end{array}$ & $\begin{array}{r}14 \\
4 \\
5 \\
4 \\
11\end{array}$ \\
\hline
\end{tabular}


TABLE 5.---Continued

\begin{tabular}{|c|c|c|c|c|c|c|c|}
\hline $\begin{array}{l}\text { LOCAL } \\
\text { IDENTIFIER }\end{array}$ & $\begin{array}{c}\text { DEPTH } \\
\text { OF } \\
\text { WELL }\end{array}$ & $\begin{array}{l}\text { BEGIN } \\
\text { YEAR }\end{array}$ & $\begin{array}{l}\text { END } \\
\text { YEAR }\end{array}$ & $\begin{array}{l}\text { MAXIMUM } \\
\text { (MICHO- } \\
\text { MHOS) }\end{array}$ & $\begin{array}{l}\text { MINIMUM } \\
\text { (MICRO- } \\
\text { MHOS) }\end{array}$ & $\begin{array}{l}\text { MEAN } \\
\text { MICRO- } \\
\text { MHOSI }\end{array}$ & $\begin{array}{l}\text { NUMEER } \\
\text { OF } \\
\text { SAMPLES }\end{array}$ \\
\hline $\begin{array}{l}25 / 44 E-03801 \\
25 / 44 E-04 R 03 \\
25 / 44 E-05001 \\
25 / 44 E-05 K 01 \\
25 / 44 E-05 R 01\end{array}$ & $\begin{array}{l}122 \\
118 \\
202 \\
234 \\
130\end{array}$ & $\begin{array}{l}1977 \\
1971 \\
1977 \\
1977 \\
1977\end{array}$ & $\begin{array}{l}1974 \\
1978 \\
1978 \\
1978 \\
1978\end{array}$ & $\begin{array}{l}515 \\
290 \\
290 \\
270 \\
320\end{array}$ & $\begin{array}{l}465 \\
267 \\
240 \\
270 \\
261\end{array}$ & $\begin{array}{l}483 \\
281 \\
270 \\
270 \\
299\end{array}$ & $\begin{array}{l}3 \\
5 \\
3 \\
2 \\
3\end{array}$ \\
\hline $\begin{array}{l}25 / 44 E-06 A 01 \\
25 / 44 E-07 B 01 \\
25 / 44 E-07 C 01 \\
25 / 44 E-07 J 02 \\
25 / 44 E-07 J 03\end{array}$ & $\begin{array}{r}104 \\
120 \\
90 \\
110 \\
-\end{array}$ & $\begin{array}{l}1955 \\
1977 \\
1973 \\
1977 \\
1979\end{array}$ & $\begin{array}{l}1978 \\
1978 \\
1974 \\
1978 \\
1981\end{array}$ & $\begin{array}{l}228 \\
350 \\
330 \\
332 \\
30 y\end{array}$ & $\begin{array}{l}204 \\
260 \\
306 \\
300 \\
284\end{array}$ & $\begin{array}{l}216 \\
320 \\
314 \\
313 \\
295\end{array}$ & $\begin{array}{r}2 \\
3 \\
7 \\
3 \\
61\end{array}$ \\
\hline $\begin{array}{l}25 / 44 E-08001 \\
25 / 44 E-08 N 01 \\
25 / 44 E-09 C 01 \\
25 / 44 E-09 C 02 \\
25 / 44 E-09 E 01\end{array}$ & $\begin{array}{r}112 \\
135 \\
125 \\
150 \\
-\end{array}$ & $\begin{array}{l}1977 \\
1978 \\
1977 \\
1977 \\
1977\end{array}$ & $\begin{array}{l}1978 \\
1978 \\
1978 \\
1978 \\
1978\end{array}$ & $\begin{array}{l}340 \\
301 \\
285 \\
310 \\
300\end{array}$ & $\begin{array}{l}271 \\
290 \\
250 \\
250 \\
270\end{array}$ & $\begin{array}{l}310 \\
295 \\
267 \\
275 \\
288\end{array}$ & $\begin{array}{r}3 \\
2 \\
3 \\
16 \\
3\end{array}$ \\
\hline $\begin{array}{l}25 / 44 E-09 P 01 \\
25 / 44 E-12 D 01 \\
25 / 44 E-12 M 01 \\
25 / 44 E-13 M 01 \\
25 / 44 E-15 E 01\end{array}$ & $\begin{array}{l}121 \\
100 \\
125 \\
156\end{array}$ & $\begin{array}{l}1977 \\
1977 \\
1978 \\
1977 \\
1942\end{array}$ & $\begin{array}{l}1978 \\
1978 \\
1978 \\
1979 \\
1978\end{array}$ & $\begin{array}{l}325 \\
320 \\
300 \\
370 \\
300\end{array}$ & $\begin{array}{l}290 \\
280 \\
280 \\
210 \\
269\end{array}$ & $\begin{array}{l}303 \\
298 \\
295 \\
275 \\
283\end{array}$ & $\begin{array}{r}10 \\
3 \\
4 \\
3 \\
4\end{array}$ \\
\hline $\begin{array}{l}25 / 44 E-15 E 02 \\
25 / 44 E-15 J 01 \\
25 / 44 E-16 E 01 \\
25 / 44 E-17 A 01 \\
25 / 44 E-17 M 01\end{array}$ & $\begin{array}{l}150 \\
156 \\
128 \\
125 \\
114\end{array}$ & $\begin{array}{l}1970 \\
1977 \\
1978 \\
1977 \\
1977\end{array}$ & $\begin{array}{l}1973 \\
1978 \\
1974 \\
1978 \\
1978\end{array}$ & $\begin{array}{l}360 \\
410 \\
286 \\
300 \\
270\end{array}$ & $\begin{array}{l}232 \\
190 \\
280 \\
285 \\
256\end{array}$ & $\begin{array}{l}265 \\
278 \\
284 \\
292 \\
265\end{array}$ & $\begin{array}{r}15 \\
3 \\
3 \\
3 \\
3\end{array}$ \\
\hline $\begin{array}{l}25 / 44 E-17 R 01 \\
25 / 44 E-18002 \\
25 / 44 E-18 F 01 \\
25 / 44 E-18 M 01 \\
25 / 44 E-19001\end{array}$ & $\begin{array}{r}125 \\
120 \\
110 \\
79 \\
88\end{array}$ & $\begin{array}{l}1977 \\
1973 \\
1977 \\
1951 \\
1973\end{array}$ & $\begin{array}{l}1978 \\
1979 \\
1978 \\
1951 \\
1977\end{array}$ & $\begin{array}{l}375 \\
285 \\
263 \\
237 \\
394\end{array}$ & $\begin{array}{l}225 \\
255 \\
221 \\
237 \\
369\end{array}$ & $\begin{array}{l}269 \\
273 \\
247 \\
237 \\
381\end{array}$ & $\begin{array}{r}16 \\
9 \\
3 \\
1 \\
5\end{array}$ \\
\hline $\begin{array}{l}25 / 44 E-20 K 01 \\
25 / 44 E-2 I J 01 \\
25 / 44 E-21 L 01 \\
25 / 44 E-21 N 01 \\
25 / 44 E-22 H 02\end{array}$ & $\begin{array}{l}129 \\
117 \\
177 \\
181 \\
160\end{array}$ & $\begin{array}{l}1977 \\
1977 \\
1977 \\
1977 \\
1977\end{array}$ & $\begin{array}{l}1978 \\
1978 \\
1978 \\
1978 \\
1978\end{array}$ & $\begin{array}{l}360 \\
340 \\
537 \\
337 \\
380\end{array}$ & $\begin{array}{l}294 \\
241 \\
247 \\
269 \\
250\end{array}$ & $\begin{array}{l}327 \\
297 \\
360 \\
297 \\
315\end{array}$ & $\begin{array}{l}2 \\
3 \\
3 \\
3 \\
2\end{array}$ \\
\hline $\begin{array}{l}25 / 44 E-22 R 01 \\
25 / 44 E-26 L 01 \\
25 / 44 E-27 E 01 \\
25 / 44 E-27 L 01 \\
25 / 44 E-28 L 01\end{array}$ & $\begin{array}{l}176 \\
166 \\
220 \\
180 \\
131\end{array}$ & $\begin{array}{l}1977 \\
1977 \\
1977 \\
1977 \\
1977\end{array}$ & $\begin{array}{l}1978 \\
1978 \\
1978 \\
1979 \\
1978\end{array}$ & $\begin{array}{l}410 \\
390 \\
355 \\
529 \\
520\end{array}$ & $\begin{array}{l}222 \\
320 \\
295 \\
378 \\
309\end{array}$ & $\begin{array}{l}316 \\
350 \\
323 \\
482 \\
443\end{array}$ & $\begin{array}{l}2 \\
3 \\
3 \\
4 \\
3\end{array}$ \\
\hline
\end{tabular}


TABLE 5.--Continued

\begin{tabular}{|c|c|c|c|c|c|c|c|}
\hline $\begin{array}{l}\text { LOCAL } \\
\text { IDENTIF IER }\end{array}$ & $\begin{array}{l}\text { DEPTH } \\
\text { OF } \\
\text { WELL }\end{array}$ & $\begin{array}{l}\text { BEGIN } \\
\text { YEAR }\end{array}$ & $\begin{array}{l}\text { END } \\
\text { YEAR }\end{array}$ & $\begin{array}{l}\text { MAXIMUM } \\
\text { (MICKO- } \\
\text { MHOS) }\end{array}$ & $\begin{array}{l}\text { MINIMUM } \\
\text { (MICRO- } \\
\text { MHOS) }\end{array}$ & $\begin{array}{l}\text { MEAN } \\
\text { MICRO- } \\
\text { MHOS I }\end{array}$ & $\begin{array}{l}\text { NUMBER } \\
\text { OF } \\
\text { SAMPLES }\end{array}$ \\
\hline $\begin{array}{l}25 / 44 E-28 P 01 \\
25 / 44 E-28 R 01 \\
25 / 44 E-29 A 01 \\
25 / 44 E-29 H 01 \\
25 / 45 E-01 H 03\end{array}$ & $\begin{array}{l}167 \\
132 \\
157 \\
154 \\
165\end{array}$ & $\begin{array}{l}1977 \\
1977 \\
1977 \\
1978 \\
1977\end{array}$ & $\begin{array}{l}1978 \\
1978 \\
1978 \\
1978 \\
1978\end{array}$ & $\begin{array}{l}493 \\
530 \\
533 \\
367 \\
112\end{array}$ & $\begin{array}{l}440 \\
374 \\
354 \\
367 \\
110\end{array}$ & $\begin{array}{l}467 \\
461 \\
444 \\
367 \\
111\end{array}$ & $\begin{array}{l}2 \\
3 \\
2 \\
1 \\
2\end{array}$ \\
\hline $\begin{array}{l}25 / 45 E-02 G 02 \\
25 / 45 E-03 M 01 \\
25 / 45 E-04 A 01 \\
25 / 45 E-05 H O I \\
25 / 45 E-05 R 02\end{array}$ & $\begin{array}{l}235 \\
138 \\
135 \\
137 \\
130\end{array}$ & $\begin{array}{l}1977 \\
1977 \\
1951 \\
1977 \\
1978\end{array}$ & $\begin{array}{l}1978 \\
1980 \\
1951 \\
1977 \\
1978\end{array}$ & $\begin{array}{l}263 \\
316 \\
297 \\
255 \\
375\end{array}$ & $\begin{array}{l}240 \\
310 \\
297 \\
255 \\
250\end{array}$ & $\begin{array}{l}249 \\
314 \\
297 \\
255 \\
326\end{array}$ & $\begin{array}{l}3 \\
3 \\
1 \\
1 \\
10\end{array}$ \\
\hline $\begin{array}{l}25 / 45 E-06003 \\
25 / 45 E-07 A 03 \\
25 / 45 E-08 R 02 \\
25 / 45 E-09 B 01 \\
25 / 45 E-10 F 01\end{array}$ & $\begin{array}{r}146 \\
170 \\
165 \\
107 \\
85\end{array}$ & $\begin{array}{l}1977 \\
1977 \\
1978 \\
1977 \\
1977\end{array}$ & $\begin{array}{l}1978 \\
1980 \\
1978 \\
1978 \\
1978\end{array}$ & $\begin{array}{l}635 \\
311 \\
205 \\
140 \\
155\end{array}$ & $\begin{array}{r}435 \\
275 \\
195 \\
135 \\
73\end{array}$ & $\begin{array}{l}505 \\
295 \\
200 \\
138 \\
110\end{array}$ & $\begin{array}{l}3 \\
4 \\
5 \\
2 \\
3\end{array}$ \\
\hline $\begin{array}{l}25 / 45 E-11 K 02 \\
25 / 45 E-14 C 01 \\
25 / 45 E-15 C 01 \\
25 / 45 E-15001 \\
25 / 45 E-15 R 01\end{array}$ & $\begin{array}{l}225 \\
238 \\
157 \\
195 \\
155\end{array}$ & $\begin{array}{l}1977 \\
1977 \\
1977 \\
1972 \\
1971\end{array}$ & $\begin{array}{l}1978 \\
1978 \\
1978 \\
1977 \\
1978\end{array}$ & $\begin{array}{l}115 \\
210 \\
243 \\
270 \\
145\end{array}$ & $\begin{array}{r}94 \\
185 \\
240 \\
184 \\
40\end{array}$ & $\begin{array}{l}105 \\
198 \\
242 \\
241 \\
116\end{array}$ & $\begin{array}{r}2 \\
2 \\
2 \\
21 \\
18\end{array}$ \\
\hline $\begin{array}{l}25 / 45 E-16 K 01 \\
25 / 45 E-17 D 02 \\
25 / 45 E-17 D 03 \\
25 / 45 E-17 P 01 \\
25 / 45 E-17 P 02\end{array}$ & $\begin{array}{l}185 \\
213 \\
215 \\
203 \\
218\end{array}$ & $\begin{array}{l}1973 \\
1977 \\
1978 \\
1977 \\
1978\end{array}$ & $\begin{array}{l}1977 \\
1977 \\
1980 \\
1977 \\
1978\end{array}$ & $\begin{array}{r}1040 \\
187 \\
170 \\
180 \\
200\end{array}$ & $\begin{array}{l}780 \\
187 \\
155 \\
180 \\
165\end{array}$ & $\begin{array}{l}860 \\
187 \\
164 \\
190 \\
183\end{array}$ & $\begin{array}{l}5 \\
1 \\
3 \\
1 \\
2\end{array}$ \\
\hline $\begin{array}{l}25 / 45 E-18 A O 1 \\
25 / 45 E-18 R O 1 \\
25 / 45 E-18 R 02 \\
26 / 42 E-02 N O 1 \\
26 / 42 E-03 E 015\end{array}$ & $\begin{array}{r}118 \\
190 \\
227 \\
29 \\
-\end{array}$ & $\begin{array}{l}1942 \\
1970 \\
1978 \\
1977 \\
1977\end{array}$ & $\begin{array}{l}1942 \\
1978 \\
1978 \\
1978 \\
1978\end{array}$ & $\begin{array}{l}183 \\
210 \\
170 \\
308 \\
205\end{array}$ & $\begin{array}{l}183 \\
120 \\
170 \\
280 \\
244\end{array}$ & $\begin{array}{l}183 \\
165 \\
170 \\
294 \\
255\end{array}$ & $\begin{array}{r}1 \\
16 \\
1 \\
2 \\
3\end{array}$ \\
\hline $\begin{array}{l}26 / 42 E-05 C 03 \\
26 / 42 E-05 E 01 \\
26 / 42 E-05 F 02 \\
25 / 42 E-06 L 01 \\
25 / 42 E-07 A 04\end{array}$ & $\begin{array}{r}22 \\
64 \\
25 \\
94 \\
126\end{array}$ & $\begin{array}{l}1977 \\
1977 \\
1977 \\
1977 \\
1977\end{array}$ & $\begin{array}{l}1978 \\
1978 \\
1978 \\
1978 \\
1978\end{array}$ & $\begin{array}{l}288 \\
495 \\
315 \\
315 \\
241\end{array}$ & $\begin{array}{l}258 \\
325 \\
272 \\
297 \\
174\end{array}$ & $\begin{array}{l}273 \\
424 \\
291 \\
306 \\
207\end{array}$ & $\begin{array}{r}3 \\
16 \\
3 \\
2 \\
3\end{array}$ \\
\hline $\begin{array}{l}26 / 42 E-07601 \\
25 / 42 E-08 A O 1 \\
26 / 42 E-08 N O 1 \\
25 / 42 E-10 F 01 \\
25 / 42 E-11 F 01\end{array}$ & $\begin{array}{r}45 \\
300 \\
58 \\
9 \\
38\end{array}$ & $\begin{array}{l}1951 \\
1977 \\
1977 \\
1977 \\
1977\end{array}$ & $\begin{array}{l}1951 \\
1980 \\
1978 \\
1978 \\
1979\end{array}$ & $\begin{array}{l}509 \\
367 \\
374 \\
350 \\
300\end{array}$ & $\begin{array}{l}569 \\
324 \\
300 \\
273 \\
280\end{array}$ & $\begin{array}{l}569 \\
341 \\
329 \\
305 \\
291\end{array}$ & $\begin{array}{l}1 \\
4 \\
3 \\
3 \\
4\end{array}$ \\
\hline
\end{tabular}


TABLE 5.--Continued

\begin{tabular}{|c|c|c|c|c|c|c|c|}
\hline $\begin{array}{l}\text { LOCAL } \\
\text { IDENTIFIER }\end{array}$ & $\begin{array}{l}\text { DEPTH } \\
\text { OF } \\
\text { WELL }\end{array}$ & $\begin{array}{l}\text { BEGIN } \\
\text { YEAR }\end{array}$ & $\begin{array}{l}\text { END } \\
\text { YEAR }\end{array}$ & $\begin{array}{l}\text { MAXIMUM } \\
\text { (MICRO- } \\
\text { MHOS) }\end{array}$ & $\begin{array}{l}\text { MINIMUM } \\
\text { (MICRO- } \\
\text { MHOS) }\end{array}$ & $\begin{array}{l}\text { MEAN } \\
\text { MICRO- } \\
\text { MHOSI }\end{array}$ & $\begin{array}{l}\text { NUMBER } \\
\text { OF } \\
\text { SAMPLES }\end{array}$ \\
\hline $\begin{array}{l}26 / 42 E-11 J 01 S \\
26 / 42 E-12 A 01 S \\
26 / 42 E-12 L O 1 \\
26 / 42 E-17 A 02 \\
26 / 42 E-20 B 01\end{array}$ & $\begin{array}{r}- \\
126 \\
72 \\
140\end{array}$ & $\begin{array}{l}1973 \\
1973 \\
1964 \\
1977 \\
1977\end{array}$ & $\begin{array}{l}1978 \\
1979 \\
1978 \\
1979 \\
1979\end{array}$ & $\begin{array}{l}367 \\
344 \\
349 \\
280 \\
350\end{array}$ & $\begin{array}{l}320 \\
287 \\
312 \\
169 \\
330\end{array}$ & $\begin{array}{l}354 \\
302 \\
330 \\
218 \\
343\end{array}$ & $\begin{array}{l}9 \\
7 \\
4 \\
4 \\
4\end{array}$ \\
\hline $\begin{array}{l}26 / 42 E-20 N 01 \\
26 / 42 E-21 F 02 \\
26 / 42 E-21 R 03 \\
25 / 42 E-23 F 01 \\
25 / 42 E-27 F 01\end{array}$ & $\begin{array}{r}159 \\
- \\
93 \\
578 \\
126\end{array}$ & $\begin{array}{l}1954 \\
1977 \\
1977 \\
1977 \\
1977\end{array}$ & $\begin{array}{l}1961 \\
1978 \\
1978 \\
1978 \\
1978\end{array}$ & $\begin{array}{l}283 \\
482 \\
820 \\
300 \\
530\end{array}$ & $\begin{array}{l}222 \\
301 \\
730 \\
239 \\
270\end{array}$ & $\begin{array}{l}271 \\
400 \\
780 \\
270 \\
425\end{array}$ & $\begin{array}{r}8 \\
3 \\
3 \\
2 \\
17\end{array}$ \\
\hline $\begin{array}{l}26 / 42 E-27 N 01 \\
26 / 42 E-27 N 02 \\
26 / 42 E-34 N 03 \\
26 / 43 E-03 N 01 \\
26 / 43 E-03 P 01\end{array}$ & $\begin{array}{r}129 \\
150 \\
71 \\
180 \\
203\end{array}$ & $\begin{array}{l}1962 \\
1962 \\
1977 \\
1977 \\
1977\end{array}$ & $\begin{array}{l}1978 \\
1962 \\
1978 \\
1978 \\
1979\end{array}$ & $\begin{array}{l}300 \\
278 \\
306 \\
539 \\
650\end{array}$ & $\begin{array}{l}269 \\
278 \\
300 \\
298 \\
300\end{array}$ & $\begin{array}{l}287 \\
278 \\
303 \\
442 \\
438\end{array}$ & $\begin{array}{l}9 \\
1 \\
2 \\
3 \\
4\end{array}$ \\
\hline $\begin{array}{l}26 / 43 E-05001 \\
26 / 43 E-05 L 01 S \\
26 / 43 E-06 G 01 \\
26 / 43 E-06 J 01 \\
26 / 43 E-07 B 01 S\end{array}$ & $\begin{array}{r}30 \\
- \\
30 \\
75 \\
-\end{array}$ & $\begin{array}{l}1977 \\
1973 \\
1977 \\
1977 \\
1973\end{array}$ & $\begin{array}{l}1978 \\
1978 \\
1978 \\
1978 \\
1977\end{array}$ & $\begin{array}{l}530 \\
445 \\
350 \\
440 \\
320\end{array}$ & $\begin{array}{l}351 \\
387 \\
260 \\
300 \\
294\end{array}$ & $\begin{array}{l}414 \\
407 \\
313 \\
386 \\
307\end{array}$ & $\begin{array}{l}3 \\
6 \\
3 \\
3 \\
5\end{array}$ \\
\hline $\begin{array}{l}26 / 43 E-07601 \\
26 / 43 E-07 K 01 \\
26 / 43 E-07 P 01 \\
26 / 43 E-08604 \\
26 / 43 E-08602\end{array}$ & $\begin{array}{r}164 \\
126 \\
90 \\
49\end{array}$ & $\begin{array}{l}1978 \\
1977 \\
1977 \\
1964 \\
1977\end{array}$ & $\begin{array}{l}1978 \\
1974 \\
1978 \\
1978 \\
1978\end{array}$ & $\begin{array}{l}420 \\
361 \\
387 \\
632 \\
710\end{array}$ & $\begin{array}{l}310 \\
249 \\
246 \\
301 \\
602\end{array}$ & $\begin{array}{l}369 \\
309 \\
308 \\
427 \\
641\end{array}$ & $\begin{array}{l}7 \\
4 \\
3 \\
4 \\
3\end{array}$ \\
\hline $\begin{array}{l}26 / 43 E-10 K 01 \\
26 / 43 E-16002 \\
26 / 43 E-16 F 02 \\
26 / 43 E-17801 \\
26 / 43 E-17 J 01\end{array}$ & $\begin{array}{l}107 \\
285 \\
268 \\
220 \\
248\end{array}$ & $\begin{array}{l}1964 \\
1977 \\
1960 \\
1978 \\
1977\end{array}$ & $\begin{array}{l}1978 \\
1978 \\
1960 \\
1978 \\
1974\end{array}$ & $\begin{array}{l}637 \\
350 \\
291 \\
340 \\
320\end{array}$ & $\begin{array}{l}472 \\
312 \\
290 \\
336 \\
304\end{array}$ & $\begin{array}{l}567 \\
334 \\
290 \\
338 \\
314\end{array}$ & $\begin{array}{l}4 \\
3 \\
2 \\
2 \\
4\end{array}$ \\
\hline $\begin{array}{l}26 / 43 E-19 A 01 \\
26 / 43 E-19 L 03 \\
26 / 43 E-20001 \\
26 / 43 E-20 N O 1 \\
26 / 43 E-21 E 02\end{array}$ & $\begin{array}{l}163 \\
- \\
286 \\
238 \\
246\end{array}$ & $\begin{array}{l}1942 \\
1977 \\
1977 \\
1977 \\
1978\end{array}$ & $\begin{array}{l}1978 \\
1978 \\
1979 \\
1978 \\
1978\end{array}$ & $\begin{array}{l}308 \\
341 \\
306 \\
270 \\
387\end{array}$ & $\begin{array}{l}243 \\
321 \\
215 \\
249 \\
387\end{array}$ & $\begin{array}{l}270 \\
332 \\
258 \\
260 \\
387\end{array}$ & $\begin{array}{l}4 \\
3 \\
4 \\
3 \\
1\end{array}$ \\
\hline $\begin{array}{l}26 / 43 E-21 R 01 \\
26 / 43 E-27 E 01 \\
26 / 43 E-28001 \\
26 / 43 E-30 F 01 \\
26 / 43 E-30 H O 1\end{array}$ & $\begin{array}{l}260 \\
258 \\
274 \\
312 \\
310\end{array}$ & $\begin{array}{l}1978 \\
1951 \\
1978 \\
1977 \\
1977\end{array}$ & $\begin{array}{l}1978 \\
1978 \\
1978 \\
1978 \\
1978\end{array}$ & $\begin{array}{l}365 \\
310 \\
350 \\
317 \\
243\end{array}$ & $\begin{array}{l}365 \\
248 \\
270 \\
310 \\
215\end{array}$ & $\begin{array}{l}365 \\
276 \\
308 \\
312 \\
233\end{array}$ & $\begin{array}{r}1 \\
4 \\
10 \\
3 \\
3\end{array}$ \\
\hline
\end{tabular}


TABLE 5.--Continued

\begin{tabular}{|c|c|c|c|c|c|c|c|}
\hline $\begin{array}{l}\text { LOCAL } \\
\text { IDENTIFIER }\end{array}$ & $\begin{array}{l}\text { DEPTH } \\
\text { OF } \\
\text { WELL }\end{array}$ & $\begin{array}{l}\text { BEGIN } \\
\text { YEAR }\end{array}$ & $\begin{array}{l}\text { END } \\
\text { YEAR }\end{array}$ & $\begin{array}{l}\text { MAXIMUM } \\
\text { (MICRO- } \\
\text { MHOS) }\end{array}$ & $\begin{array}{l}\text { MINIMUM } \\
\text { (MICRO- } \\
\text { MHOS) }\end{array}$ & $\begin{array}{l}\text { MEAN } \\
\text { MICKO- } \\
\text { MHOS }\end{array}$ & $\begin{array}{l}\text { NUMEER } \\
\text { OF } \\
\text { SAMPLES }\end{array}$ \\
\hline $\begin{array}{l}26 / 43 E-30 R 02 \\
26 / 43 E-31 A 01 \\
26 / 43 E-34 P 01 \\
26 / 44 E-32 R 01 \\
26 / 45 E-25 J 01\end{array}$ & $\begin{array}{l}293 \\
270 \\
210 \\
113 \\
263\end{array}$ & $\begin{array}{l}1964 \\
1977 \\
1977 \\
1955 \\
1977\end{array}$ & $\begin{array}{l}1978 \\
1978 \\
1978 \\
1955 \\
1978\end{array}$ & $\begin{array}{l}263 \\
242 \\
340 \\
358 \\
300\end{array}$ & $\begin{array}{l}235 \\
208 \\
276 \\
358 \\
225\end{array}$ & $\begin{array}{l}245 \\
225 \\
319 \\
358 \\
289\end{array}$ & $\begin{array}{r}4 \\
3 \\
3 \\
1 \\
15\end{array}$ \\
\hline $\begin{array}{l}26 / 45 E-33 N 01 \\
26 / 45 E-34 L 01 \\
26 / 45 E-34 L 03 \\
25 / 45 E-35 F 01 \\
26 / 45 E-35 F 02\end{array}$ & $\begin{array}{l}120 \\
198 \\
212 \\
232 \\
223\end{array}$ & $\begin{array}{l}1978 \\
1978 \\
1977 \\
1973 \\
1977\end{array}$ & $\begin{array}{l}1978 \\
1978 \\
1978 \\
1978 \\
1978\end{array}$ & $\begin{array}{l}340 \\
240 \\
255 \\
277 \\
270\end{array}$ & $\begin{array}{l}320 \\
240 \\
220 \\
230 \\
245\end{array}$ & $\begin{array}{l}327 \\
240 \\
238 \\
262 \\
258\end{array}$ & $\begin{array}{l}3 \\
1 \\
2 \\
5 \\
2\end{array}$ \\
\hline $\begin{array}{l}26 / 45 E-36 E 01 \\
26 / 45 E-36 N 01 \\
26 / 45 E-36 Q 01 \\
26 / 46 E-30001 \\
26 / 46 E-30 M 01\end{array}$ & $\begin{array}{r}149 \\
145 \\
190 \\
140\end{array}$ & $\begin{array}{l}1942 \\
1973 \\
1973 \\
1977 \\
1978\end{array}$ & $\begin{array}{l}1942 \\
1974 \\
1974 \\
1978 \\
1978\end{array}$ & $\begin{array}{l}295 \\
305 \\
279 \\
300 \\
210\end{array}$ & $\begin{array}{l}295 \\
296 \\
274 \\
250 \\
210\end{array}$ & $\begin{array}{l}295 \\
301 \\
276 \\
262 \\
210\end{array}$ & $\begin{array}{r}1 \\
3 \\
3 \\
16 \\
1\end{array}$ \\
\hline $\begin{array}{l}26 / 46 E-31 M 01 \\
26 / 46 E-31 M 04 \\
26 / 46 E-31 M 05 \\
26 / 46 E-31 M 06\end{array}$ & $\begin{array}{l}249 \\
223 \\
222 \\
184\end{array}$ & $\begin{array}{l}1970 \\
1977 \\
1978 \\
1978\end{array}$ & $\begin{array}{l}1978 \\
1977 \\
1978 \\
1978\end{array}$ & $\begin{array}{l}315 \\
242 \\
315 \\
350\end{array}$ & $\begin{array}{l}208 \\
242 \\
230 \\
280\end{array}$ & $\begin{array}{l}240 \\
242 \\
272 \\
308\end{array}$ & $\begin{array}{r}15 \\
1 \\
2 \\
10\end{array}$ \\
\hline
\end{tabular}


TABLE 6.--Summary of nitrate concentrations in ground water samples from the Spokane Valley

\begin{tabular}{|c|c|c|c|c|c|c|c|}
\hline IDENTIFIER & $\begin{array}{l}\text { DEPTH } \\
\text { OF } \\
\text { WELL }\end{array}$ & $\begin{array}{l}\text { HEGIN } \\
\text { YEAR }\end{array}$ & $\begin{array}{l}\text { END } \\
\text { YEAR }\end{array}$ & $\begin{array}{l}\text { MAXIMUM } \\
\text { NITRATE } \\
\text { (MG/L } \\
\text { AS N) }\end{array}$ & $\begin{array}{l}\text { MINIMUM } \\
\text { NITRATE } \\
\text { (MG/L } \\
\text { AS N) }\end{array}$ & $\begin{array}{l}\text { MEAN } \\
\text { NITRATE } \\
\text { MG/L } \\
\text { AS NI }\end{array}$ & $\begin{array}{l}\text { NUMBER } \\
\text { OF } \\
\text { SAMPLES }\end{array}$ \\
\hline $\begin{array}{l}25 / 42 E-03 H 01 \\
25 / 42 E-11 E 01 \\
25 / 42 E-11 E 02 \\
25 / 42 E-11 M 02 \\
25 / 42 E-13501\end{array}$ & $\begin{array}{l}124 \\
201 \\
52 \\
230 \\
200\end{array}$ & $\begin{array}{l}1977 \\
1947 \\
1948 \\
1955 \\
1942\end{array}$ & $\begin{array}{l}1978 \\
1979 \\
1961 \\
1905 \\
1978\end{array}$ & $\begin{array}{l}1.70 \\
1.40 \\
1.08 \\
1.11 \\
2.40\end{array}$ & $\begin{array}{l}1.20 \\
0.29 \\
0.23 \\
0.84 \\
1.30\end{array}$ & $\begin{array}{l}1.43 \\
0.74 \\
0.48 \\
1.02 \\
1.85\end{array}$ & $\begin{array}{r}3 \\
17 \\
13 \\
8 \\
10\end{array}$ \\
\hline $\begin{array}{l}25 / 42 E-14 J 01 \\
25 / 42 E-23 \mathrm{~S} 01 \\
25 / 43 E-04 \mathrm{BO} 2 \\
25 / 43 E-08 A 01 \\
25 / 43 E-08 D 01\end{array}$ & $\begin{array}{r}160 \\
59 \\
227 \\
124 \\
-\end{array}$ & $\begin{array}{l}1977 \\
1977 \\
1922 \\
1978 \\
1942\end{array}$ & $\begin{array}{l}1977 \\
1978 \\
1980 \\
1980 \\
1942\end{array}$ & $\begin{array}{l}0.76 \\
1.80 \\
3.61 \\
1.40 \\
0.99\end{array}$ & $\begin{array}{l}0.76 \\
1.20 \\
1.10 \\
1.10 \\
0.99\end{array}$ & $\begin{array}{l}0.76 \\
1.50 \\
2.27 \\
1.30 \\
0.99\end{array}$ & $\begin{array}{l}1 \\
3 \\
3 \\
3 \\
1\end{array}$ \\
\hline $\begin{array}{l}25 / 43 E-09601 \\
25 / 43 E-09603 \\
25 / 43 E-10 P 02 \\
25 / 43 E-11603 \\
25 / 43 E-11002\end{array}$ & $\begin{array}{r}32 \\
73 \\
96 \\
128 \\
80\end{array}$ & $\begin{array}{l}1951 \\
1977 \\
1977 \\
1977 \\
1977\end{array}$ & $\begin{array}{l}1951 \\
1978 \\
1978 \\
1978 \\
1978\end{array}$ & $\begin{array}{l}0.09 \\
0.72 \\
2.10 \\
0.41 \\
0.46\end{array}$ & $\begin{array}{l}0.09 \\
0.24 \\
1.20 \\
0.17 \\
0.00\end{array}$ & $\begin{array}{l}0.09 \\
0.45 \\
1.53 \\
0.26 \\
0.19\end{array}$ & $\begin{array}{r}1 \\
3 \\
15 \\
3 \\
3\end{array}$ \\
\hline $\begin{array}{l}25 / 43 E-11 E 02 \\
25 / 43 E-11 G 01 \\
25 / 43 E-11 G 04 \\
25 / 43 E-11 J 03 \\
25 / 43 E-11 M 03\end{array}$ & $\begin{array}{r}- \\
37 \\
44 \\
125 \\
98\end{array}$ & $\begin{array}{l}1977 \\
1939 \\
1962 \\
1960 \\
1977\end{array}$ & $\begin{array}{l}1977 \\
1939 \\
1962 \\
1962 \\
1978\end{array}$ & $\begin{array}{l}0.67 \\
0.72 \\
1.04 \\
0.97 \\
0.94\end{array}$ & $\begin{array}{l}0.67 \\
0.72 \\
1.04 \\
0.90 \\
0.80\end{array}$ & $\begin{array}{l}0.67 \\
0.72 \\
1.04 \\
0.94 \\
0.89\end{array}$ & $\begin{array}{r}1 \\
1 \\
1 \\
2 \\
16\end{array}$ \\
\hline $\begin{array}{l}25 / 43 E-11 N 01 \\
25 / 43 E-11 R 01 \\
25 / 43 E-12 H O 1 \\
25 / 43 E-12 L 02 \\
25 / 43 E-13 A O 1\end{array}$ & $\begin{array}{r}70 \\
104 \\
94 \\
100 \\
110\end{array}$ & $\begin{array}{l}1977 \\
1977 \\
1971 \\
1977 \\
1973\end{array}$ & $\begin{array}{l}1978 \\
1978 \\
1978 \\
1978 \\
1978\end{array}$ & $\begin{array}{l}1.00 \\
1.50 \\
1.80 \\
1.40 \\
1.40\end{array}$ & $\begin{array}{l}0.90 \\
1.30 \\
0.68 \\
1.30 \\
0.84\end{array}$ & $\begin{array}{l}0.95 \\
1.40 \\
1.26 \\
1.35 \\
1.08\end{array}$ & $\begin{array}{r}3 \\
3 \\
18 \\
2 \\
9\end{array}$ \\
\hline $\begin{array}{l}25 / 43 E-13 \mathrm{HO} \\
25 / 43 E-13 R 01 \\
25 / 43 E-14 E 01 \\
25 / 43 E-14 K O 1 \\
25 / 43 E-14 L O 1\end{array}$ & $\begin{array}{r}71 \\
140 \\
211 \\
83 \\
120\end{array}$ & $\begin{array}{l}1974 \\
1977 \\
1977 \\
1973 \\
1977\end{array}$ & $\begin{array}{l}1974 \\
1977 \\
1978 \\
1978 \\
1978\end{array}$ & $\begin{array}{l}1.00 \\
1.40 \\
1.30 \\
1.40 \\
1.50\end{array}$ & $\begin{array}{l}1.00 \\
1.40 \\
1.10 \\
0.90 \\
1.30\end{array}$ & $\begin{array}{l}1.00 \\
1.40 \\
1.21 \\
1.17 \\
1.40\end{array}$ & $\begin{array}{r}1 \\
1 \\
16 \\
9 \\
14\end{array}$ \\
\hline $\begin{array}{l}25 / 43 E-15 G 02 \\
25 / 43 E-16 K 01 \\
25 / 43 E-17 K 01 \\
25 / 43 E-21 B 01 \\
25 / 43 E-22 F 01\end{array}$ & $\begin{array}{r}129 \\
65 \\
100 \\
87 \\
77\end{array}$ & $\begin{array}{l}1977 \\
1977 \\
1977 \\
1977 \\
1977\end{array}$ & $\begin{array}{l}1978 \\
1978 \\
1978 \\
1977 \\
1978\end{array}$ & $\begin{array}{l}1.50 \\
1.70 \\
3.90 \\
2.80 \\
4.20\end{array}$ & $\begin{array}{l}1.30 \\
1.40 \\
3.50 \\
2.80 \\
3.70\end{array}$ & $\begin{array}{l}1.40 \\
1.53 \\
3.73 \\
2.80 \\
4.03\end{array}$ & $\begin{array}{l}3 \\
3 \\
3 \\
1 \\
3\end{array}$ \\
\hline $\begin{array}{l}25 / 43 E-23 A 01 \\
25 / 43 E-23 A 02 \\
25 / 43 E-24601 \\
25 / 44 E-01 J 01 \\
25 / 44 E-01 R 01\end{array}$ & $\begin{array}{l}142 \\
150 \\
144 \\
160 \\
150\end{array}$ & $\begin{array}{l}1970 \\
1972 \\
1971 \\
1973 \\
1977\end{array}$ & $\begin{array}{l}1979 \\
1973 \\
1973 \\
1974 \\
1978\end{array}$ & $\begin{array}{l}9.20 \\
3.50 \\
5.60 \\
2.10 \\
1.20\end{array}$ & $\begin{array}{l}1.40 \\
1.60 \\
2.30 \\
0.71 \\
0.88\end{array}$ & $\begin{array}{l}2.84 \\
2.52 \\
3.64 \\
1.11 \\
0.98\end{array}$ & $\begin{array}{r}20 \\
15 \\
14 \\
4 \\
5\end{array}$ \\
\hline
\end{tabular}


TABLE 6.--Continued

\begin{tabular}{|c|c|c|c|c|c|c|c|}
\hline IDENTIF IER & $\begin{array}{l}\text { DEPTH } \\
\text { OF } \\
\text { WELL }\end{array}$ & $\begin{array}{l}\text { BEGIN } \\
\text { YEAR }\end{array}$ & $\begin{array}{l}\text { ENU } \\
\text { YEAR }\end{array}$ & $\begin{array}{l}\text { MAXIMUM } \\
\text { NITRATE } \\
\text { (MG/L } \\
\text { AS N) }\end{array}$ & $\begin{array}{l}\text { MINIMUM } \\
\text { NITRATE } \\
\text { (MG/L } \\
\text { AS N) }\end{array}$ & $\begin{array}{l}\text { MEAN } \\
\text { NITRATE } \\
\text { MG/L } \\
\text { AS NI }\end{array}$ & $\begin{array}{l}\text { NUMBER } \\
\text { OF } \\
\text { SAMPLES }\end{array}$ \\
\hline $\begin{array}{l}25 / 44 E-02 B 01 \\
25 / 44 E-02001 \\
25 / 44 E-03801 \\
25 / 44 E-04 R 03\end{array}$ & $\begin{array}{l}127 \\
129 \\
122 \\
118\end{array}$ & $\begin{array}{l}1955 \\
1973 \\
1977 \\
1971\end{array}$ & $\begin{array}{l}1978 \\
1978 \\
1979 \\
1978\end{array}$ & $\begin{array}{l}3.40 \\
5.40 \\
6.50 \\
1.50\end{array}$ & $\begin{array}{l}1.54 \\
1.40 \\
4.30 \\
1.33\end{array}$ & $\begin{array}{l}2.38 \\
2.68 \\
5.53 \\
1.41\end{array}$ & $\begin{array}{l}4 \\
6 \\
3 \\
4\end{array}$ \\
\hline $\begin{array}{l}25 / 44 E-05001 \\
25 / 44 E-05 K 01 \\
25 / 44 E-05 R 01 \\
25 / 44 E-06401 \\
25 / 44 E-07801\end{array}$ & $\begin{array}{l}202 \\
234 \\
130 \\
104 \\
120\end{array}$ & $\begin{array}{l}1977 \\
1977 \\
1977 \\
1955 \\
1977\end{array}$ & $\begin{array}{l}1978 \\
1978 \\
1978 \\
1978 \\
1978\end{array}$ & $\begin{array}{l}2.60 \\
1.80 \\
1.50 \\
1.80 \\
1.30\end{array}$ & $\begin{array}{l}2.20 \\
1.70 \\
1.20 \\
1.13 \\
1.20\end{array}$ & $\begin{array}{l}2.43 \\
1.75 \\
1.30 \\
1.40 \\
1.27\end{array}$ & $\begin{array}{l}3 \\
2 \\
3 \\
2 \\
3\end{array}$ \\
\hline $\begin{array}{l}25 / 44 E-07 C 01 \\
25 / 44 E-07 J 02 \\
25 / 44 E-07 J 03 \\
25 / 44 E-08001 \\
25 / 44 E-08 N 01\end{array}$ & $\begin{array}{r}90 \\
110 \\
112 \\
135\end{array}$ & $\begin{array}{l}1973 \\
1977 \\
1979 \\
1977 \\
1978\end{array}$ & $\begin{array}{l}1979 \\
1978 \\
1981 \\
1978 \\
1978\end{array}$ & $\begin{array}{l}1.40 \\
1.20 \\
1.40 \\
1.60 \\
1.60\end{array}$ & $\begin{array}{l}0.76 \\
1.10 \\
0.86 \\
1.30 \\
1.00\end{array}$ & $\begin{array}{l}1.07 \\
1.17 \\
1.05 \\
1.43 \\
1.30\end{array}$ & $\begin{array}{r}7 \\
3 \\
67 \\
3 \\
2\end{array}$ \\
\hline $\begin{array}{l}25 / 44 E-09 C 01 \\
25 / 44 E-09 C 02 \\
25 / 44 E-09 E 01 \\
25 / 44 E-09 P 01 \\
25 / 44 E-12001\end{array}$ & $\begin{array}{r}125 \\
150 \\
121 \\
-\end{array}$ & $\begin{array}{l}1977 \\
1977 \\
1977 \\
1977 \\
1977\end{array}$ & $\begin{array}{l}1978 \\
1978 \\
1978 \\
1978 \\
1978\end{array}$ & $\begin{array}{l}1.20 \\
1.20 \\
0.84 \\
1.00 \\
1.20\end{array}$ & $\begin{array}{l}1.10 \\
1.00 \\
0.82 \\
0.84 \\
1.00\end{array}$ & $\begin{array}{l}1.13 \\
1.11 \\
0.83 \\
0.93 \\
1.10\end{array}$ & $\begin{array}{r}3 \\
16 \\
3 \\
16 \\
3\end{array}$ \\
\hline $\begin{array}{l}25 / 44 E-12 M 01 \\
25 / 44 E-13 M O 1 \\
25 / 44 E-15 E 01 \\
25 / 44 E-15 E 02 \\
25 / 44 E-15 J 01\end{array}$ & $\begin{array}{l}100 \\
125 \\
156 \\
150 \\
156\end{array}$ & $\begin{array}{l}1978 \\
1977 \\
1942 \\
1970 \\
1977\end{array}$ & $\begin{array}{l}1978 \\
1979 \\
1978 \\
1973 \\
1978\end{array}$ & $\begin{array}{l}0.33 \\
2.60 \\
1.20 \\
2.50 \\
2.60\end{array}$ & $\begin{array}{l}0.73 \\
1.00 \\
0.54 \\
0.16 \\
0.76\end{array}$ & $\begin{array}{l}0.79 \\
1.73 \\
1.01 \\
1.05 \\
1.65\end{array}$ & $\begin{array}{r}5 \\
3 \\
4 \\
15 \\
3\end{array}$ \\
\hline $\begin{array}{l}25 / 44 E-16 E 01 \\
25 / 44 E-17 A 01 \\
25 / 44 E-17 M 01 \\
25 / 44 E-17 R 01 \\
25 / 44 E-18002\end{array}$ & $\begin{array}{l}128 \\
125 \\
114 \\
125 \\
120\end{array}$ & $\begin{array}{l}1978 \\
1977 \\
1977 \\
1977 \\
1973\end{array}$ & $\begin{array}{l}1979 \\
1978 \\
1978 \\
1978 \\
1979\end{array}$ & $\begin{array}{l}1.40 \\
1.20 \\
1.80 \\
2.40 \\
1.40\end{array}$ & $\begin{array}{l}1.10 \\
1.00 \\
1.30 \\
1.20 \\
0.91\end{array}$ & $\begin{array}{l}1.30 \\
1.10 \\
1.50 \\
1.55 \\
1.25\end{array}$ & $\begin{array}{r}3 \\
3 \\
3 \\
16 \\
9\end{array}$ \\
\hline $\begin{array}{l}25 / 44 E-18 F 01 \\
25 / 44 E-18 M 01 \\
25 / 44 E-19001 \\
25 / 44 E-20 K 01 \\
25 / 44 E-21 J 01\end{array}$ & $\begin{array}{r}110 \\
79 \\
88 \\
129 \\
117\end{array}$ & $\begin{array}{l}1977 \\
1951 \\
1973 \\
1977 \\
1977\end{array}$ & $\begin{array}{l}1978 \\
1951 \\
1977 \\
1978 \\
1978\end{array}$ & $\begin{array}{l}1.40 \\
1.17 \\
3.70 \\
2.70 \\
2.30\end{array}$ & $\begin{array}{l}1.10 \\
1.17 \\
2.20 \\
2.60 \\
1.50\end{array}$ & $\begin{array}{l}1.23 \\
1.17 \\
2.86 \\
2.65 \\
1.87\end{array}$ & $\begin{array}{l}3 \\
1 \\
5 \\
2 \\
3\end{array}$ \\
\hline $\begin{array}{l}25 / 44 E-21 L 01 \\
25 / 44 E-21 N 01 \\
25 / 44 E-22 H 02 \\
25 / 44 E-22 K 01 \\
25 / 44 E-26 L 01\end{array}$ & $\begin{array}{l}177 \\
181 \\
160 \\
176 \\
166\end{array}$ & $\begin{array}{l}1977 \\
1977 \\
1977 \\
1977 \\
1977\end{array}$ & $\begin{array}{l}1978 \\
1978 \\
1978 \\
1978 \\
1978\end{array}$ & $\begin{array}{l}3.20 \\
3.50 \\
2.60 \\
2.80 \\
2.80\end{array}$ & $\begin{array}{l}1.70 \\
2.00 \\
1.60 \\
1.60 \\
2.50\end{array}$ & $\begin{array}{l}2.63 \\
2.63 \\
2.10 \\
2.20 \\
2.70\end{array}$ & $\begin{array}{l}3 \\
3 \\
2 \\
2 \\
3\end{array}$ \\
\hline
\end{tabular}


TABLE 6.--Continued

\begin{tabular}{|c|c|c|c|c|c|c|c|}
\hline IDENTIFIER & $\begin{array}{l}\text { DEPTH } \\
\text { OF } \\
\text { WELL }\end{array}$ & $\begin{array}{l}\text { BEGIN } \\
\text { YEAR }\end{array}$ & $\begin{array}{l}\text { END } \\
\text { YEAR }\end{array}$ & $\begin{array}{l}\text { MAXIMUM } \\
\text { NITRATE } \\
\text { (MG/L } \\
\text { AS N) }\end{array}$ & $\begin{array}{l}\text { MINIMUM } \\
\text { NITRATE } \\
\text { (MG/L } \\
\text { AS N) }\end{array}$ & $\begin{array}{l}\text { MEAN } \\
\text { NITRATE } \\
\text { MG/L } \\
\text { AS NI }\end{array}$ & $\begin{array}{l}\text { NUMBER } \\
\text { OF } \\
\text { SAMPLES }\end{array}$ \\
\hline $\begin{array}{l}25 / 44 E-27 E 01 \\
25 / 44 E-27 L 01 \\
25 / 44 E-28 L 01 \\
25 / 44 E-28 P 01\end{array}$ & $\begin{array}{l}220 \\
180 \\
131 \\
167\end{array}$ & $\begin{array}{l}1977 \\
1977 \\
1977 \\
1977\end{array}$ & $\begin{array}{l}1978 \\
1979 \\
1978 \\
1978\end{array}$ & $\begin{array}{l}2.60 \\
5.70 \\
6.00 \\
5.80\end{array}$ & $\begin{array}{l}2.10 \\
3.70 \\
5.60 \\
5.60\end{array}$ & $\begin{array}{l}2.40 \\
4.75 \\
5.90 \\
5.70\end{array}$ & $\begin{array}{l}3 \\
4 \\
3 \\
2\end{array}$ \\
\hline $\begin{array}{l}25 / 44 E-28 K 01 \\
25 / 44 E-29 A 01 \\
25 / 44 E-29 H 01 \\
25 / 45 E-01 H 03 \\
25 / 45 E-026 \cap 2\end{array}$ & $\begin{array}{l}132 \\
157 \\
154 \\
165 \\
235\end{array}$ & $\begin{array}{l}1977 \\
1977 \\
1978 \\
1977 \\
1977\end{array}$ & $\begin{array}{l}1978 \\
1978 \\
1978 \\
1978 \\
1978\end{array}$ & $\begin{array}{l}5.70 \\
5.60 \\
3.70 \\
0.74 \\
1.00\end{array}$ & $\begin{array}{l}3.70 \\
3.60 \\
3.70 \\
0.70 \\
0.89\end{array}$ & $\begin{array}{l}4.73 \\
4.60 \\
3.70 \\
0.72 \\
0.93\end{array}$ & $\begin{array}{l}3 \\
2 \\
1 \\
2 \\
3\end{array}$ \\
\hline $\begin{array}{l}25 / 45 E-03 M 01 \\
25 / 45 E-04 A 01 \\
25 / 45 E-05 H 01 \\
25 / 45 E-05 R 02 \\
25 / 45 E-06003\end{array}$ & $\begin{array}{l}138 \\
135 \\
137 \\
130 \\
146\end{array}$ & $\begin{array}{l}1977 \\
1951 \\
1977 \\
1978 \\
1977\end{array}$ & $\begin{array}{l}1980 \\
1951 \\
1977 \\
1978 \\
1978\end{array}$ & $\begin{array}{l}1.10 \\
1.22 \\
0.84 \\
1.40 \\
6.00\end{array}$ & $\begin{array}{l}0.94 \\
1.22 \\
0.84 \\
1.20 \\
4.40\end{array}$ & $\begin{array}{l}1.05 \\
1.22 \\
0.84 \\
1.27 \\
5.20\end{array}$ & $\begin{array}{r}3 \\
1 \\
1 \\
10 \\
3\end{array}$ \\
\hline $\begin{array}{l}25 / 45 E-07 A 03 \\
25 / 45 E-08 R 02 \\
25 / 45 E-09601 \\
25 / 45 E-10 F 01 \\
25 / 45 E-11 K 02\end{array}$ & $\begin{array}{r}170 \\
165 \\
107 \\
85 \\
225\end{array}$ & $\begin{array}{l}1977 \\
1978 \\
1977 \\
1977 \\
1977\end{array}$ & $\begin{array}{l}1980 \\
1978 \\
1978 \\
1978 \\
1978\end{array}$ & $\begin{array}{l}1.10 \\
1.00 \\
0.79 \\
0.95 \\
1.00\end{array}$ & $\begin{array}{l}0.83 \\
0.76 \\
0.72 \\
0.35 \\
0.64\end{array}$ & $\begin{array}{l}0.94 \\
0.84 \\
0.75 \\
0.52 \\
0.82\end{array}$ & $\begin{array}{l}4 \\
5 \\
2 \\
3 \\
2\end{array}$ \\
\hline $\begin{array}{l}25 / 45 E-14 C 01 \\
25 / 45 E-15 C 01 \\
25 / 45 E-15001 \\
25 / 45 E-15 R 01 \\
25 / 45 E-16 K 01\end{array}$ & $\begin{array}{l}238 \\
157 \\
195 \\
155 \\
185\end{array}$ & $\begin{array}{l}1977 \\
1977 \\
1972 \\
1971 \\
1973\end{array}$ & $\begin{array}{l}1978 \\
1978 \\
1977 \\
1978 \\
1977\end{array}$ & $\begin{array}{l}2.80 \\
2.50 \\
3.50 \\
1.80 \\
1.60\end{array}$ & $\begin{array}{l}2.40 \\
1.50 \\
0.45 \\
0.01 \\
0.95\end{array}$ & $\begin{array}{l}2.60 \\
2.00 \\
1.93 \\
0.81 \\
1.17\end{array}$ & $\begin{array}{r}2 \\
2 \\
21 \\
19 \\
5\end{array}$ \\
\hline $\begin{array}{l}25 / 45 E-17002 \\
25 / 45 E-17003 \\
25 / 45 E-17 P 01 \\
25 / 45 E-17 P 02 \\
25 / 45 E-18 A 01\end{array}$ & $\begin{array}{l}213 \\
215 \\
203 \\
218 \\
118\end{array}$ & $\begin{array}{l}1977 \\
1978 \\
1977 \\
1978 \\
1942\end{array}$ & $\begin{array}{l}1977 \\
1980 \\
1977 \\
1978 \\
1942\end{array}$ & $\begin{array}{l}0.81 \\
0.82 \\
1.50 \\
1.40 \\
0.52\end{array}$ & $\begin{array}{l}0.81 \\
0.66 \\
1.50 \\
1.30 \\
0.52\end{array}$ & $\begin{array}{l}0.81 \\
0.76 \\
1.50 \\
1.35 \\
0.52\end{array}$ & $\begin{array}{l}1 \\
3 \\
1 \\
2 \\
1\end{array}$ \\
\hline $\begin{array}{l}25 / 45 E-18 R 01 \\
25 / 45 E-18 R 02 \\
26 / 42 E-02 N 01 \\
26 / 42 E-03 E 015 \\
26 / 42 E-05 C 03\end{array}$ & $\begin{array}{r}190 \\
227 \\
29 \\
- \\
22\end{array}$ & $\begin{array}{l}1970 \\
1978 \\
1977 \\
1977 \\
1977\end{array}$ & $\begin{array}{l}1978 \\
1978 \\
1978 \\
1978 \\
1978\end{array}$ & $\begin{array}{l}2.00 \\
0.89 \\
0.24 \\
0.91 \\
0.35\end{array}$ & $\begin{array}{l}0.05 \\
0.89 \\
0.01 \\
0.82 \\
0.20\end{array}$ & $\begin{array}{l}0.97 \\
0.89 \\
0.12 \\
0.86 \\
0.29\end{array}$ & $\begin{array}{r}16 \\
1 \\
2 \\
3 \\
3\end{array}$ \\
\hline $\begin{array}{l}25 / 42 E-05 E 01 \\
25 / 42 E-05 F 02 \\
26 / 42 E-06 L 01 \\
25 / 42 E-07 A 04 \\
26 / 42 E-07 G 01\end{array}$ & $\begin{array}{r}64 \\
25 \\
94 \\
126 \\
45\end{array}$ & $\begin{array}{l}1977 \\
1977 \\
1977 \\
1977 \\
1951\end{array}$ & $\begin{array}{l}1978 \\
1978 \\
1978 \\
1978 \\
1951\end{array}$ & $\begin{array}{l}1.60 \\
1.60 \\
1.40 \\
0.85 \\
7.23\end{array}$ & $\begin{array}{l}0.00 \\
0.05 \\
1.20 \\
0.34 \\
7.23\end{array}$ & $\begin{array}{l}1.07 \\
0.57 \\
1.30 \\
0.52 \\
7.23\end{array}$ & $\begin{array}{r}16 \\
3 \\
2 \\
3 \\
1\end{array}$ \\
\hline
\end{tabular}


TABLE 6.---Continued

\begin{tabular}{|c|c|c|c|c|c|c|c|}
\hline IOENT IF IEP & $\begin{array}{l}\text { DEPTH } \\
\text { OF } \\
\text { WELL }\end{array}$ & $\begin{array}{l}\text { AEGIN } \\
\text { YEAR }\end{array}$ & $\begin{array}{l}\text { END } \\
\text { YEAR }\end{array}$ & $\begin{array}{l}\text { MAXIMUM } \\
\text { NITRATE } \\
\text { (MG/L } \\
\text { AS N) }\end{array}$ & $\begin{array}{l}\text { MINIMUM } \\
\text { NITRATE } \\
\text { (MG/L } \\
\text { AS N) }\end{array}$ & $\begin{array}{l}\text { MEAN } \\
\text { NITPATE } \\
\text { MG/L } \\
\text { AS N) }\end{array}$ & $\begin{array}{l}\text { NUMBER } \\
\text { OF } \\
\text { SAMPLES }\end{array}$ \\
\hline $\begin{array}{l}26 / 42 E-08 \mathrm{AOI} \\
26 / 42 E-08 \mathrm{NOI} \\
26 / 42 E-10 \mathrm{~F} 01 \\
26 / 42 E-11 \mathrm{FO} 01\end{array}$ & $\begin{array}{r}300 \\
58 \\
9 \\
38\end{array}$ & $\begin{array}{l}1977 \\
1977 \\
1977 \\
1977\end{array}$ & $\begin{array}{l}1980 \\
1978 \\
1978 \\
1979\end{array}$ & $\begin{array}{l}0.00 \\
2.80 \\
0.10 \\
1.40\end{array}$ & $\begin{array}{l}0.00 \\
2.50 \\
0.04 \\
1.30\end{array}$ & $\begin{array}{l}0.00 \\
2.63 \\
0.06 \\
1.37\end{array}$ & $\begin{array}{l}4 \\
3 \\
3 \\
4\end{array}$ \\
\hline $\begin{array}{l}26 / 42 E-11 J 01 S \\
26 / 42 E-12 A 01 S \\
26 / 42 E-12 L 01 \\
26 / 42 E-17 A 02 \\
26 / 42 E-20 B 01\end{array}$ & $\begin{array}{r}- \\
126 \\
72 \\
140\end{array}$ & $\begin{array}{l}1973 \\
1973 \\
1964 \\
1977 \\
1977\end{array}$ & $\begin{array}{l}1978 \\
1979 \\
1978 \\
1979 \\
1979\end{array}$ & $\begin{array}{l}2.70 \\
1.40 \\
2.70 \\
0.01 \\
2.40\end{array}$ & $\begin{array}{l}2.00 \\
0.92 \\
2.17 \\
0.00 \\
1.90\end{array}$ & $\begin{array}{l}2.42 \\
1.22 \\
2.44 \\
0.00 \\
2.20\end{array}$ & $\begin{array}{l}9 \\
7 \\
4 \\
4 \\
4\end{array}$ \\
\hline $\begin{array}{l}26 / 42 E-20 N 01 \\
26 / 42 E-21 F 02 \\
26 / 42 E-21 R 03 \\
25 / 42 E-23 P 01 \\
26 / 42 E-27 F 01\end{array}$ & $\begin{array}{r}159 \\
- \\
93 \\
578 \\
126\end{array}$ & $\begin{array}{l}1954 \\
1977 \\
1977 \\
1977 \\
1977\end{array}$ & $\begin{array}{l}1961 \\
1978 \\
1978 \\
1978 \\
1978\end{array}$ & $\begin{array}{l}2.01 \\
2.80 \\
3.50 \\
0.00 \\
4.50\end{array}$ & $\begin{array}{l}0.05 \\
2.20 \\
3.40 \\
0.00 \\
3.20\end{array}$ & $\begin{array}{l}1.33 \\
2.43 \\
3.47 \\
0.00 \\
3.98\end{array}$ & $\begin{array}{r}8 \\
3 \\
3 \\
2 \\
17\end{array}$ \\
\hline $\begin{array}{l}26 / 42 E-27 N 01 \\
26 / 42 E-27 N 02 \\
26 / 42 E-34 N 03 \\
26 / 43 E-03 N 01 \\
25 / 43 E-03 N 01\end{array}$ & $\begin{array}{r}129 \\
150 \\
71 \\
180 \\
203\end{array}$ & $\begin{array}{l}1962 \\
1962 \\
1977 \\
1977 \\
1977\end{array}$ & $\begin{array}{l}1978 \\
1962 \\
1978 \\
1970 \\
1979\end{array}$ & $\begin{array}{l}4.97 \\
4.74 \\
1.70 \\
8.40 \\
8.80\end{array}$ & $\begin{array}{l}0.97 \\
4.74 \\
1.70 \\
1.80 \\
1.40\end{array}$ & $\begin{array}{l}1.80 \\
4.74 \\
1.70 \\
4.13 \\
4.45\end{array}$ & $\begin{array}{l}9 \\
1 \\
2 \\
3 \\
4\end{array}$ \\
\hline $\begin{array}{l}26 / 43 E-05001 \\
26 / 43 E-05 L 01 S \\
26 / 43 E-06 G 01 \\
26 / 43 E-06 J 01 \\
26 / 43 E-076015\end{array}$ & $\begin{array}{r}30 \\
- \\
30 \\
75 \\
-\end{array}$ & $\begin{array}{l}1977 \\
1973 \\
1964 \\
1977 \\
1973\end{array}$ & $\begin{array}{l}1978 \\
1978 \\
1978 \\
1978 \\
1977\end{array}$ & $\begin{array}{l}0.80 \\
1.80 \\
1.50 \\
1.50 \\
1.60\end{array}$ & $\begin{array}{l}0.34 \\
0.95 \\
1.33 \\
1.40 \\
0.85\end{array}$ & $\begin{array}{l}0.58 \\
1.34 \\
1.46 \\
1.43 \\
1.19\end{array}$ & $\begin{array}{l}3 \\
6 \\
4 \\
3 \\
5\end{array}$ \\
\hline $\begin{array}{l}26 / 43 E-07601 \\
26 / 43 E-07 K 01 \\
26 / 43 E-07 P 01 \\
25 / 43 E-08 B 04 \\
26 / 43 E-08602\end{array}$ & $\begin{array}{r}- \\
164 \\
126 \\
90 \\
49\end{array}$ & $\begin{array}{l}1978 \\
1977 \\
1977 \\
1964 \\
1977\end{array}$ & $\begin{array}{l}1978 \\
1979 \\
1978 \\
1978 \\
1978\end{array}$ & $\begin{array}{l}2.00 \\
2.20 \\
2.20 \\
8.40 \\
3.20\end{array}$ & $\begin{array}{l}1.50 \\
1.60 \\
1.60 \\
1.76 \\
2.80\end{array}$ & $\begin{array}{l}1.73 \\
1.87 \\
1.80 \\
3.47 \\
3.03\end{array}$ & $\begin{array}{l}3 \\
4 \\
3 \\
4 \\
3\end{array}$ \\
\hline $\begin{array}{l}26 / 43 E-10 K 01 \\
26 / 43 E-16002 \\
26 / 43 E-16 F 02 \\
25 / 43 E-17 B 01 \\
26 / 43 E-17 J 01\end{array}$ & $\begin{array}{l}107 \\
285 \\
268 \\
220 \\
248\end{array}$ & $\begin{array}{l}1964 \\
1977 \\
1960 \\
1978 \\
1977\end{array}$ & $\begin{array}{l}1978 \\
1978 \\
1960 \\
1978 \\
1979\end{array}$ & $\begin{array}{l}8.30 \\
2.60 \\
1.13 \\
1.80 \\
1.90\end{array}$ & $\begin{array}{l}5.42 \\
1.70 \\
1.13 \\
1.70 \\
1.20\end{array}$ & $\begin{array}{l}7.80 \\
2.30 \\
1.13 \\
1.75 \\
1.55\end{array}$ & $\begin{array}{l}4 \\
3 \\
1 \\
2 \\
4\end{array}$ \\
\hline $\begin{array}{l}26 / 43 E-19 A 01 \\
25 / 43 E-19 L 03 \\
25 / 43 E-20001 \\
25 / 43 E-20 N 01 \\
26 / 43 E-21 E 02\end{array}$ & $\begin{array}{r}163 \\
- \\
286 \\
238 \\
246\end{array}$ & $\begin{array}{l}1942 \\
1977 \\
1977 \\
1977 \\
1978\end{array}$ & $\begin{array}{l}1978 \\
1978 \\
1979 \\
1978 \\
1978\end{array}$ & $\begin{array}{l}2.20 \\
2.30 \\
2.10 \\
1.50 \\
2.40\end{array}$ & $\begin{array}{l}1.00 \\
1.90 \\
1.00 \\
1.40 \\
2.40\end{array}$ & $\begin{array}{l}1.33 \\
2.10 \\
1.32 \\
1.43 \\
2.40\end{array}$ & $\begin{array}{l}4 \\
3 \\
4 \\
3 \\
1\end{array}$ \\
\hline
\end{tabular}


TABLE 6.--Continued

\begin{tabular}{|c|c|c|c|c|c|c|c|}
\hline IDENTIFIER & $\begin{array}{l}\text { DEPTH } \\
\text { OF } \\
\text { WELL }\end{array}$ & $\begin{array}{l}\text { HEGIN } \\
\text { YEAR }\end{array}$ & $\begin{array}{l}\text { END } \\
\text { YEAR }\end{array}$ & $\begin{array}{l}\text { MAXIMUM } \\
\text { NITRATE } \\
\text { (MG/L } \\
\text { AS N) }\end{array}$ & $\begin{array}{l}\text { MINIMUM } \\
\text { NITRATE } \\
\text { (MG/L } \\
\text { AS N) }\end{array}$ & $\begin{array}{l}\text { MEAN } \\
\text { NITRATE } \\
\text { MG/L } \\
\text { AS N I }\end{array}$ & $\begin{array}{l}\text { NUMEER } \\
\text { OF } \\
\text { SAMPLES }\end{array}$ \\
\hline $\begin{array}{l}25 / 43 E-21 \times 01 \\
26 / 43 E-27 E 0 I \\
25 / 43 E-28 Q 01 \\
26 / 43 E-30 F 01\end{array}$ & $\begin{array}{l}260 \\
258 \\
274 \\
312\end{array}$ & $\begin{array}{l}1978 \\
1951 \\
1978 \\
1977\end{array}$ & $\begin{array}{l}1978 \\
1978 \\
1978 \\
1978\end{array}$ & $\begin{array}{l}0.91 \\
1.70 \\
2.60 \\
2.40\end{array}$ & $\begin{array}{l}0.91 \\
0.88 \\
1.10 \\
1.80\end{array}$ & $\begin{array}{l}0.91 \\
1.25 \\
1.68 \\
2.10\end{array}$ & $\begin{array}{r}1 \\
4 \\
10 \\
3\end{array}$ \\
\hline $\begin{array}{l}26 / 43 E-30 H 01 \\
26 / 43 E-30 R 02 \\
25 / 43 E-31 A 01 \\
26 / 43 E-34 P 01 \\
26 / 44 E-32 R 01\end{array}$ & $\begin{array}{l}310 \\
293 \\
270 \\
210 \\
113\end{array}$ & $\begin{array}{l}1977 \\
1964 \\
1977 \\
1977 \\
1955\end{array}$ & $\begin{array}{l}1978 \\
1978 \\
1978 \\
1978 \\
1955\end{array}$ & $\begin{array}{l}0.89 \\
1.50 \\
1.10 \\
1.30 \\
2.48\end{array}$ & $\begin{array}{l}0.79 \\
1.04 \\
1.00 \\
0.00 \\
2.48\end{array}$ & $\begin{array}{l}0.86 \\
1.31 \\
1.07 \\
0.59 \\
2.48\end{array}$ & $\begin{array}{l}3 \\
4 \\
3 \\
3 \\
1\end{array}$ \\
\hline $\begin{array}{l}26 / 45 E-25 J 0 l \\
26 / 45 E-33 N 0 l \\
26 / 45 E-34 L 0 l \\
26 / 45 E-34 L 03 \\
25 / 45 E-35 F 01\end{array}$ & $\begin{array}{l}263 \\
120 \\
198 \\
212 \\
232\end{array}$ & $\begin{array}{l}1977 \\
1978 \\
1978 \\
1977 \\
1973\end{array}$ & $\begin{array}{l}1978 \\
1978 \\
1978 \\
1978 \\
1978\end{array}$ & $\begin{array}{l}0.70 \\
1.80 \\
0.82 \\
0.75 \\
0.69\end{array}$ & $\begin{array}{l}0.56 \\
0.88 \\
0.82 \\
0.74 \\
0.43\end{array}$ & $\begin{array}{l}0.63 \\
1.49 \\
0.82 \\
0.74 \\
0.56\end{array}$ & $\begin{array}{r}15 \\
3 \\
1 \\
2 \\
5\end{array}$ \\
\hline $\begin{array}{l}26 / 45 E-35 F 02 \\
26 / 45 E-36 E 0 I \\
25 / 45 E-36 N 0 I \\
26 / 45 E-36001 \\
26 / 46 E-30001\end{array}$ & $\begin{array}{r}223 \\
149 \\
145 \\
190\end{array}$ & $\begin{array}{l}1977 \\
1942 \\
1973 \\
1973 \\
1977\end{array}$ & $\begin{array}{l}1978 \\
1942 \\
1974 \\
1974 \\
1978\end{array}$ & $\begin{array}{l}0.64 \\
0.38 \\
0.87 \\
1.00 \\
1.20\end{array}$ & $\begin{array}{l}0.62 \\
0.38 \\
0.62 \\
0.94 \\
0.88\end{array}$ & $\begin{array}{l}0.63 \\
0.38 \\
0.75 \\
0.98 \\
0.98\end{array}$ & $\begin{array}{r}2 \\
1 \\
3 \\
3 \\
16\end{array}$ \\
\hline $\begin{array}{l}26 / 46 E-30 M 01 \\
26 / 46 E-31 M 01 \\
26 / 46 E-31 M 04 \\
26 / 46 E-31 M 05 \\
25 / 46 E-31 M 06\end{array}$ & $\begin{array}{l}140 \\
249 \\
223 \\
222 \\
184\end{array}$ & $\begin{array}{l}1978 \\
1970 \\
1977 \\
1978 \\
1978\end{array}$ & $\begin{array}{l}1978 \\
1973 \\
1977 \\
1978 \\
1978\end{array}$ & $\begin{array}{l}0.57 \\
1.40 \\
0.94 \\
1.10 \\
1.70\end{array}$ & $\begin{array}{l}0.57 \\
0.06 \\
0.94 \\
1.10 \\
1.10\end{array}$ & $\begin{array}{l}0.57 \\
0.85 \\
0.94 \\
1.10 \\
1.37\end{array}$ & $\begin{array}{r}1 \\
14 \\
1 \\
2 \\
10\end{array}$ \\
\hline
\end{tabular}




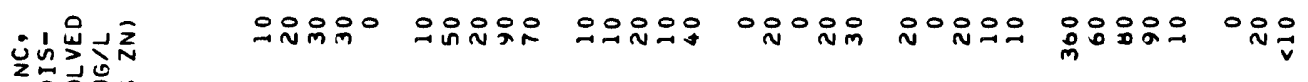

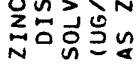

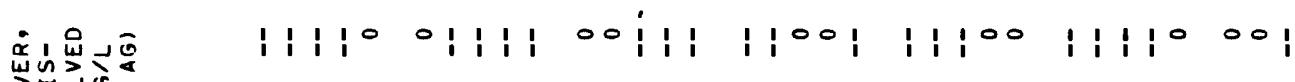
in

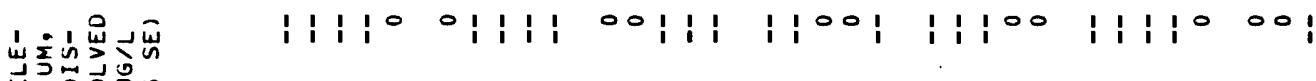

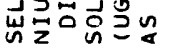

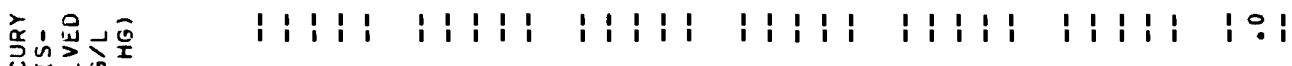

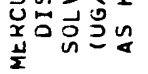

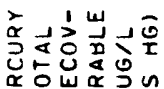

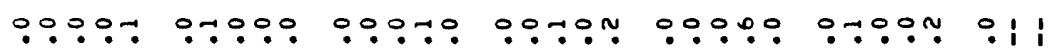
$\dot{a}$

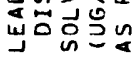

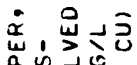

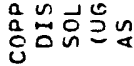

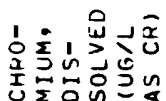

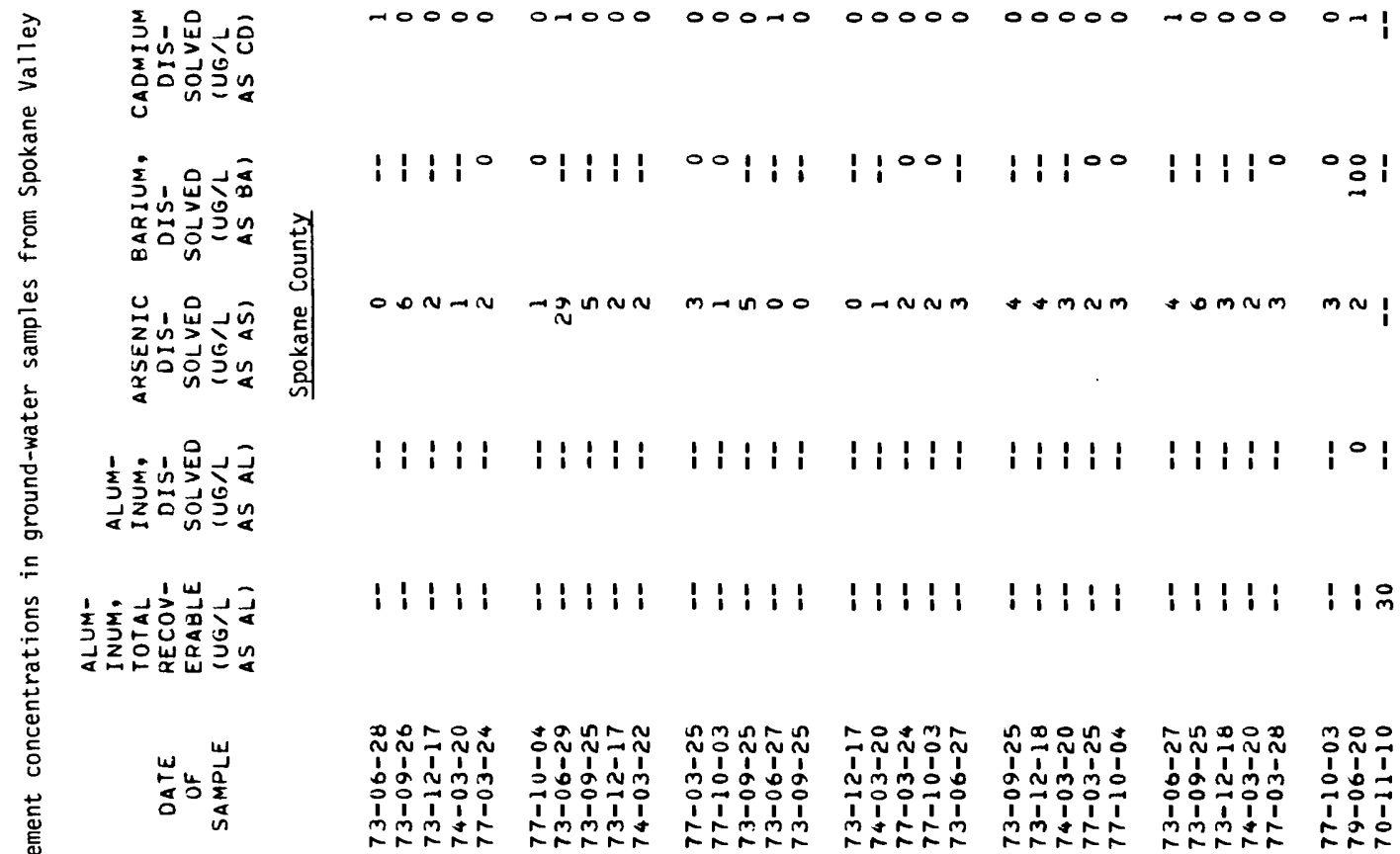

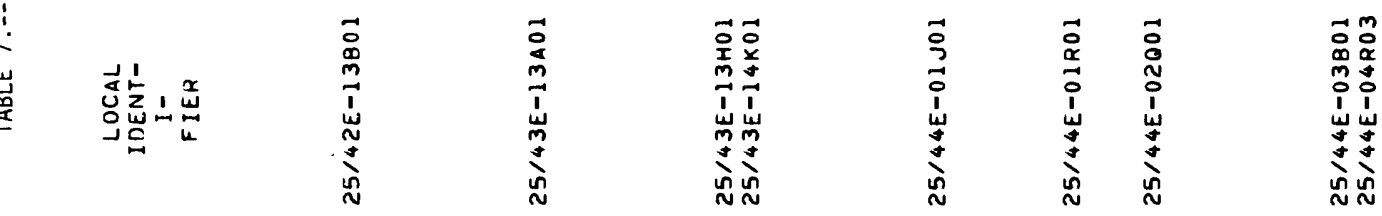




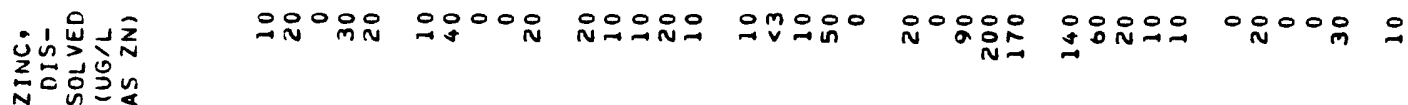

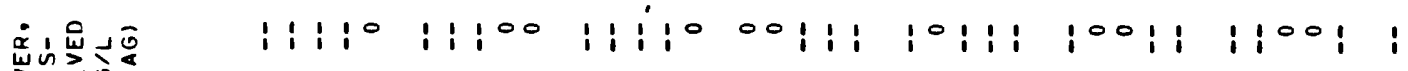

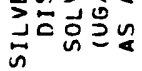

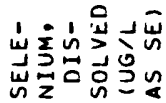

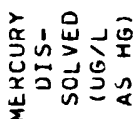

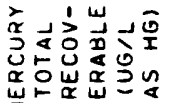

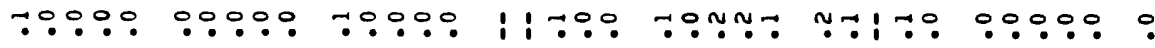

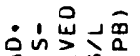

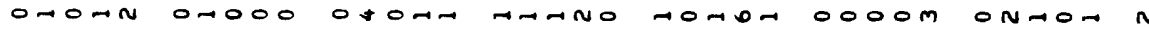

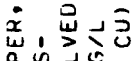

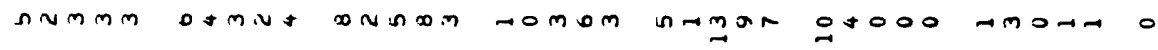

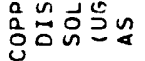

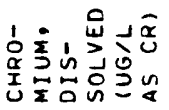

000000000000000000000000000000000000

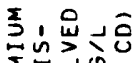

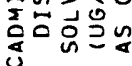

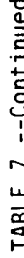

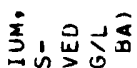

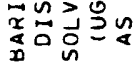

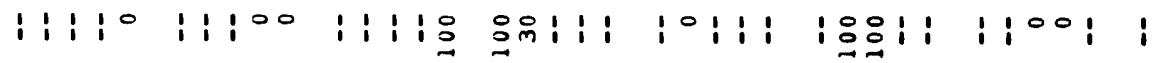

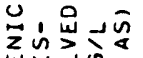

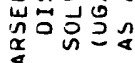

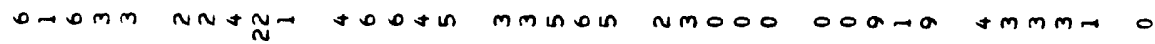

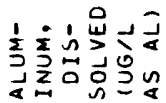

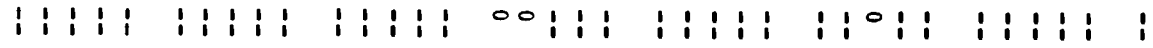

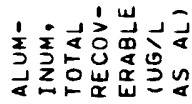

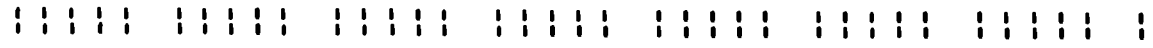

بّل

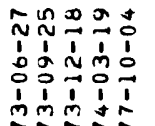

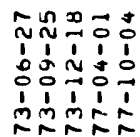

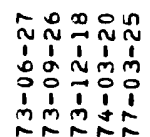

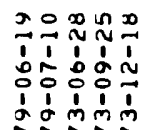

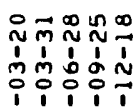

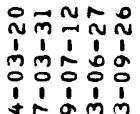

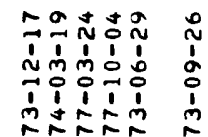

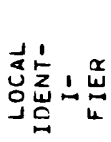

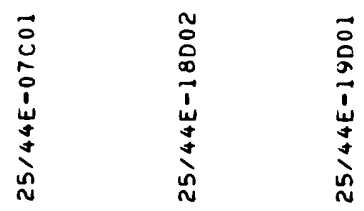

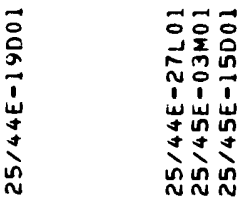

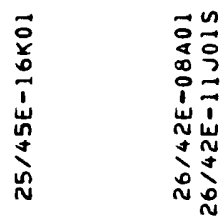

$\stackrel{n}{0}$ 


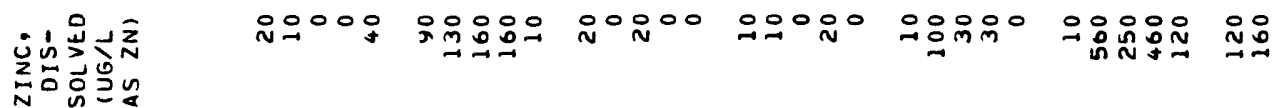

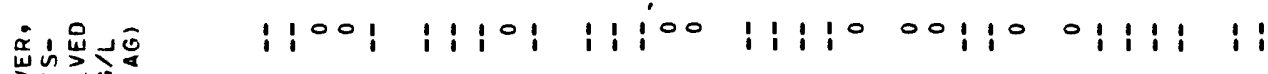

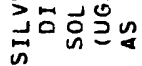

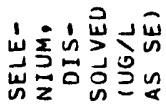

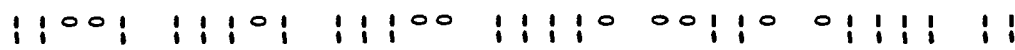

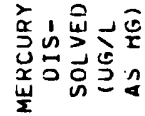

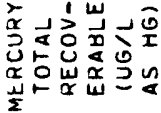

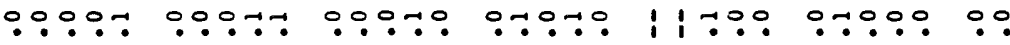

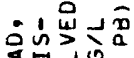

岃口苔气跑

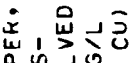

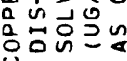

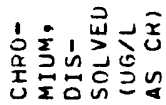

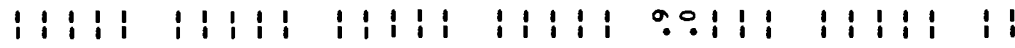

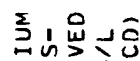

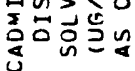

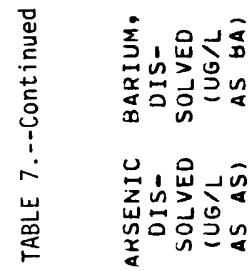

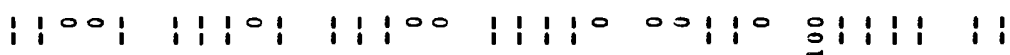

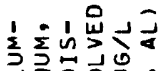

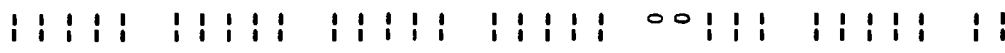

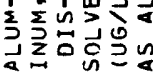

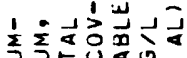

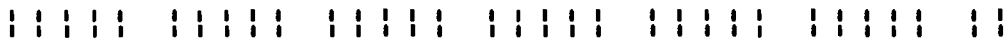

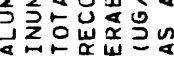

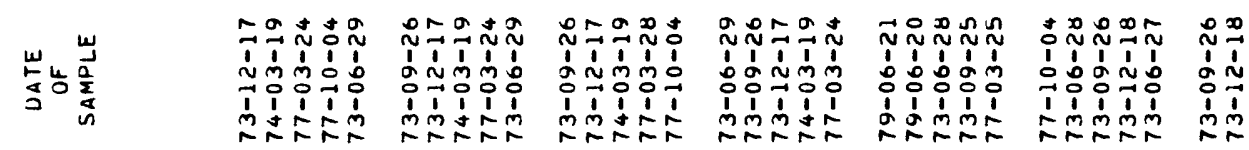

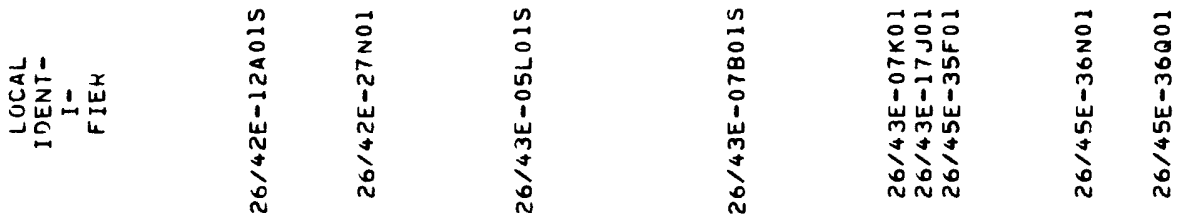


TABLE 8.--Summary of trace-element concentrations in ground-water samples from the Spokane Valley

[Values in micrograms per liter]

\begin{tabular}{lrrrr}
\hline \multicolumn{1}{c}{ Constituent } & $\begin{array}{c}\text { Number of } \\
\text { sample sites }\end{array}$ & \multicolumn{3}{c}{ Trace element concentrations } \\
\cline { 4 - 6 } Maximum & Minimum & Median \\
\hline Total aluminum & 22 & 100 & 0 & 0 \\
Dissolved aluminum & 6 & 0 & 0 & 0 \\
Dissolved arsenic & 26 & 64 & 0 & 3.5 \\
Dissolved barium & 22 & 100 & 0 & 0 \\
Dissolved cadmium & 26 & 3 & 0 & .2 \\
Dissolved chromium & 27 & $<30$ & 0 & 0 \\
Dissolved copper & 27 & 280 & 0 & 3.3 \\
Dissolved lead & 27 & $<100$ & 0 & 1.5 \\
Total mercury & 20 & .6 & 0 & .03 \\
Dissolved mercury & 6 & .9 & 0 & 0 \\
Dissolved selenium & 22 & 1 & 0 & 0 \\
Dissolved silver & 22 & 0 & 0 & 0 \\
Dissolved zinc & 27 & 560 & 0 & 16 \\
& & & & \\
\hline
\end{tabular}

'For calculation of medians, values expressed as "less than" were treated as absolute values. 
TABLE 9.--Phenol and methylene-blue-active-substance concentrations in ground-water samples from the Spokane Valley

\begin{tabular}{|c|c|c|c|c|c|c|c|}
\hline $\begin{array}{l}\text { LOCAL } \\
\text { IDENT- } \\
\text { I- } \\
\text { FIER }\end{array}$ & $\begin{array}{c}\text { DATE } \\
\text { OF } \\
\text { SAMPLE }\end{array}$ & $\begin{array}{l}\text { PHENOLS } \\
\text { (UG/L) }\end{array}$ & $\begin{array}{l}\text { METHY- } \\
\text { LENE } \\
\text { BLUE } \\
\text { ACTIVE } \\
\text { SUB- } \\
\text { STANCE } \\
\text { (MG/L) }\end{array}$ & $\begin{array}{l}\text { LOCAL } \\
\text { IDENT- } \\
\text { I - } \\
\text { FIER }\end{array}$ & $\begin{array}{c}\text { DATE } \\
\text { OF } \\
\text { SAMPLE }\end{array}$ & $\begin{array}{l}\text { PHENOLS } \\
\text { (UG/L) }\end{array}$ & $\begin{array}{l}\text { METHY- } \\
\text { LENE } \\
\text { BLUE } \\
\text { ACTIVE } \\
\text { SUB- } \\
\text { STANCE } \\
\text { (MG/L) }\end{array}$ \\
\hline
\end{tabular}

\begin{tabular}{|c|c|c|c|c|c|c|c|}
\hline $2 S / 42 E-13 R 01$ & $\begin{array}{l}73-06-28 \\
73-09-26 \\
73-12-17 \\
74-03-20 \\
77-113-24\end{array}$ & $\begin{array}{l}0 \\
1 \\
0 \\
0 \\
?\end{array}$ & $\begin{array}{l}.00 \\
.03 \\
.02 \\
.01 \\
.00\end{array}$ & $25 / 45 E-15 \cap 01$ & $\begin{array}{l}73-06-28 \\
73-09-25 \\
73-12-18 \\
74-03-20 \\
77-03-31\end{array}$ & $\begin{array}{l}0 \\
4 \\
0 \\
0 \\
0\end{array}$ & $\begin{array}{r}.00 \\
.04 \\
.02 \\
.00\end{array}$ \\
\hline $25 / 43 E-13 A 01$ & $\begin{array}{l}77-10-04 \\
73-06-29 \\
73-119-25 \\
73-12-17 \\
74-03-22\end{array}$ & $\begin{array}{l}? \\
0 \\
0 \\
0 \\
0\end{array}$ & $\begin{array}{l}.- \\
.00 \\
.10 \\
.00 \\
.00\end{array}$ & $25 / 4 \mathrm{bE}-16 \mathrm{KO} 1$ & $\begin{array}{l}73-06-28 \\
73-09-25 \\
73-12-18 \\
74-03-20 \\
77-03-31\end{array}$ & $\begin{array}{l}1 \\
3 \\
1 \\
0 \\
3\end{array}$ & $\begin{array}{l}.00 \\
.05 \\
.04 \\
.04 \\
.10\end{array}$ \\
\hline $\begin{array}{l}25 / 43 t-13 H 01 \\
25 / 41 E-14 K 01\end{array}$ & $\begin{array}{l}77-1) 3-25 \\
77-10-03 \\
73-09-25 \\
73-06-27 \\
73-09-25\end{array}$ & $\begin{array}{l}? \\
4 \\
0 \\
? \\
0\end{array}$ & $\begin{array}{r}.00 \\
-10 \\
.10 \\
.00 \\
.00\end{array}$ & $\begin{array}{r}26 / 4 C E-08 A O 1 \\
26 / 42 E-11 J 01 S\end{array}$ & $\begin{array}{l}79-07-12 \\
73-06-27 \\
73-09-26 \\
73-12-17 \\
74-03-19\end{array}$ & $\begin{array}{l}1 \\
5 \\
1 \\
0 \\
0\end{array}$ & $\begin{array}{l}.0 \\
.00 \\
.00 \\
.02 \\
.03\end{array}$ \\
\hline $25 / 44 E-61.101$ & $\begin{array}{l}73-12-17 \\
74-03-20 \\
77-03-24 \\
77-10-03 \\
73-06-27\end{array}$ & $\begin{array}{l}0 \\
0 \\
1 \\
4 \\
0\end{array}$ & $\begin{array}{l}.03 \\
.00 \\
.00 \\
.0 \\
.00\end{array}$ & $2 h / 42 t-12401 S$ & $\begin{array}{l}77-03-24 \\
77-10-04 \\
73-06-29 \\
73-09-26 \\
73-12-17\end{array}$ & $\begin{array}{l}1 \\
5 \\
3 \\
4 \\
0\end{array}$ & $\begin{array}{l}.00 \\
.- \\
.00 \\
.00 \\
.03\end{array}$ \\
\hline $23 / 44 t-31+01$ & $\begin{array}{l}73-09-23 \\
73-12-18 \\
74-0,3-2.0 \\
77-63-23\end{array}$ & $\begin{array}{l}0 \\
0 \\
0 \\
2\end{array}$ & $\begin{array}{l}.06 \\
.01 \\
.04 \\
.00\end{array}$ & & $\begin{array}{l}74-03-14 \\
77-03-24 \\
17-10-04 \\
79-0.0-21\end{array}$ & $\begin{array}{l}0 \\
1 \\
4 \\
0\end{array}$ & $\begin{array}{l}.02 \\
.00 \\
-. \\
--\end{array}$ \\
\hline & $77-10-104$ & 5 & -- & $2 n / 4(t-2|N|) \mid$ & $\begin{array}{l}73-06-29 \\
73-09-26\end{array}$ & $\begin{array}{r}12 \\
1\end{array}$ & $\begin{array}{l}.0 \\
.00 \\
.04\end{array}$ \\
\hline $25 / 44 t-0<1,01$ & $\begin{array}{l}13-10-27 \\
73-19-20 \\
73-12-18 \\
74-03-20 \\
77-03-24\end{array}$ & $\begin{array}{l}7 \\
0 \\
0 \\
0 \\
0\end{array}$ & $\begin{array}{l}.03 \\
.04 \\
.02 \\
.04 \\
.00\end{array}$ & $26 / 43 t-i) \supset L 015$ & $\begin{array}{l}73-12-17 \\
74-03-19 \\
77-03-24 \\
73-06-24 \\
73-09-26\end{array}$ & $\begin{array}{l}0 \\
0 \\
1 \\
? \\
0\end{array}$ & $\begin{array}{l}.02 \\
.00 \\
.00 \\
.00 \\
.00\end{array}$ \\
\hline $2 b / 44 t-07(0)$ & $\begin{array}{l}19-06-20 \\
73-06-27 \\
73-09-25 \\
73-12-18 \\
74-03-19\end{array}$ & $\begin{array}{l}0 \\
0 \\
? \\
0 \\
0\end{array}$ & $\begin{array}{l}.00 \\
.00 \\
.00 \\
.03 \\
.00\end{array}$ & $26 / 43 E-07401 S$ & $\begin{array}{l}73-12-17 \\
74-03-19 \\
77-03-28 \\
77-10-04 \\
73-06-29\end{array}$ & $\begin{array}{l}0 \\
0 \\
0 \\
3 \\
4\end{array}$ & $\begin{array}{l}.05 \\
.03 \\
.00 \\
-. \\
.00\end{array}$ \\
\hline $2 b / 44 t-18010 c$ & $\begin{array}{l}77-10-04 \\
73-06-27\end{array}$ & $\begin{array}{l}3 \\
?\end{array}$ &.$\overline{0}$ & & $\begin{array}{l}73-09-26 \\
73-12-17\end{array}$ & $\begin{array}{l}1 \\
0\end{array}$ &.$\overline{0}$ \\
\hline & $\begin{array}{l}73-09-25 \\
73-12-18 \\
77-04-01 \\
77-10-04\end{array}$ & $\begin{array}{l}0 \\
0 \\
2 \\
1\end{array}$ & $\begin{array}{l}.08 \\
.06 \\
.00 \\
.-\end{array}$ & $26 / 43 E-17 J 01$ & $\begin{array}{l}74-03-19 \\
77-03-24 \\
79-06-20\end{array}$ & $\begin{array}{l}0 \\
1 \\
0\end{array}$ & $\begin{array}{l}.00 \\
.10 \\
-\end{array}$ \\
\hline & $19-0 n-20$ & 0 & -- & $26 / 4 \mathrm{DE}-35 \mathrm{FOL}$ & $\begin{array}{l}73-06-28 \\
13-09-25\end{array}$ & $\begin{array}{l}4 \\
0\end{array}$ & $\begin{array}{l}.00 \\
.04\end{array}$ \\
\hline $23 / 44 t-1$ yा101 & $\begin{array}{l}73-116-27 \\
73-09-26 \\
73-12-18 \\
74-03-20 \\
77-0,3-25\end{array}$ & $\begin{array}{l}0 \\
? \\
0 \\
0 \\
?\end{array}$ & $\begin{array}{l}.00 \\
.04 \\
.04 \\
.03 \\
.00\end{array}$ & $26 / 45 t-36001$ & $\begin{array}{l}77-013-25 \\
77-10-04 \\
73-06-28 \\
73-09-26 \\
73-12-18 \\
73-06-27 \\
73-09-20 \\
73-12-18\end{array}$ & $\begin{array}{l}1 \\
1 \\
0 \\
2 \\
0\end{array}$ & $\begin{array}{l}.00 \\
.0 \\
.00 \\
.00 \\
.00 \\
.00 \\
.03 \\
.00\end{array}$ \\
\hline
\end{tabular}




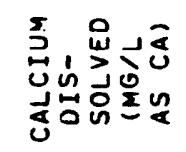

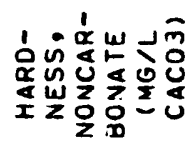

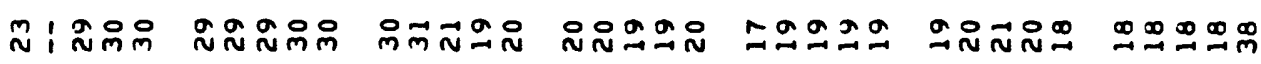 \\ 000000000000000000000000000000000000
}

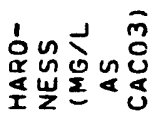

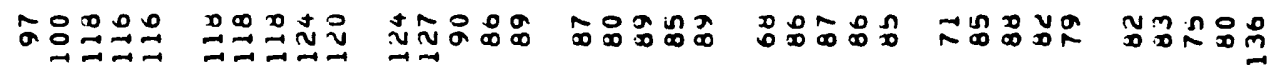

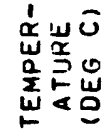
|

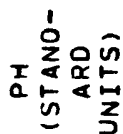

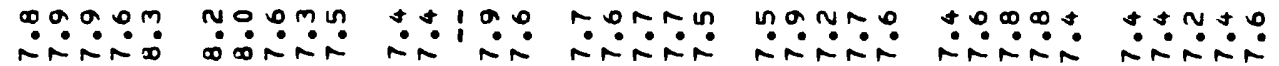

品它额

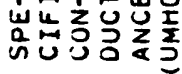

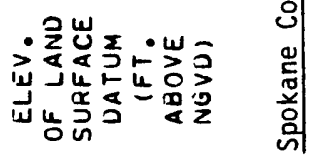

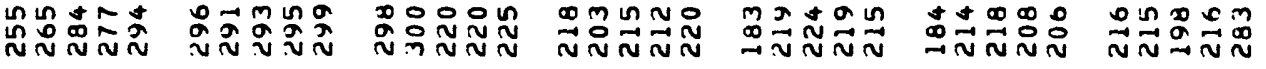

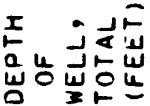

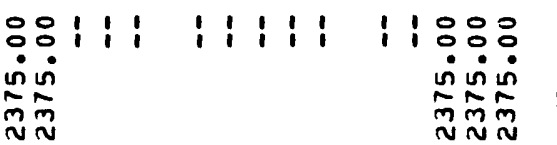

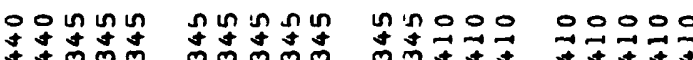

$\therefore: 8::$ vivining $\sin \sin (10$

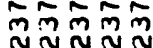

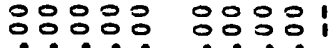

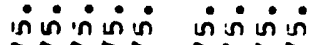

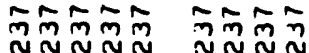

욱우우으

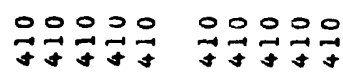

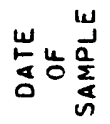

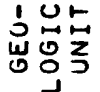

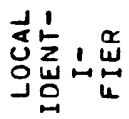

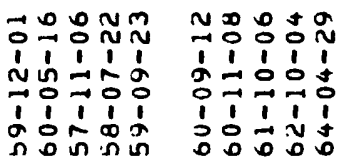

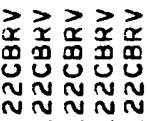

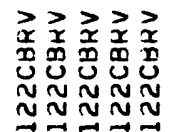

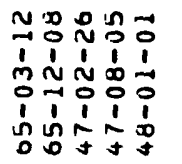

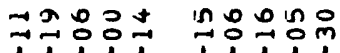

1ำ11 11111

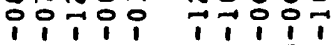

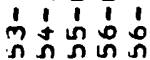

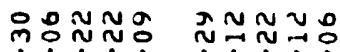

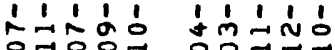

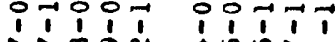

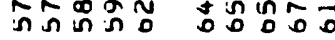

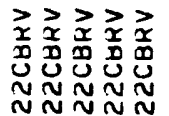

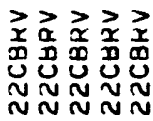

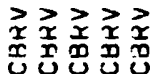
UUNU⿺辶寸

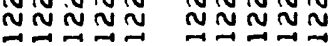

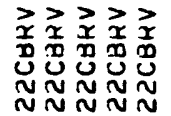
$\vec{x} \underset{x}{\vec{x}} \vec{x} \geq$ $\sim \sim N N N$

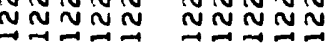




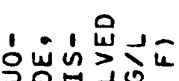

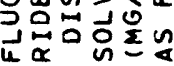

d่ 嵌

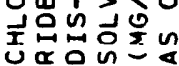

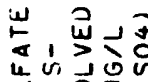
灵台别是

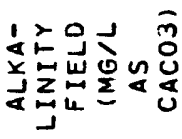

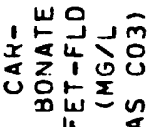

安㟔可」

造造得设

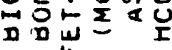

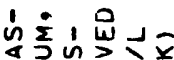

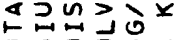

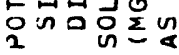

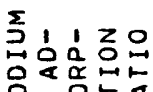

in 造战

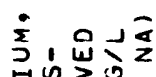

옹무워

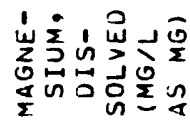

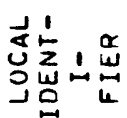

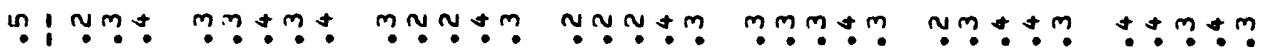
mி

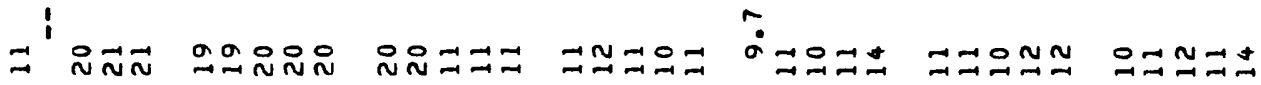

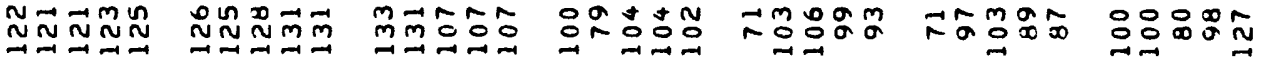
$0000 N 000000000000000000000000000000$

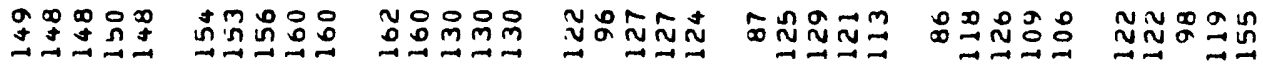

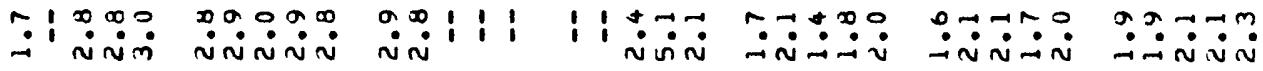

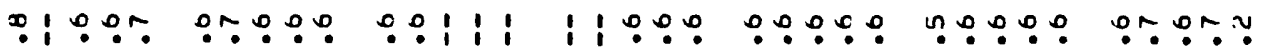

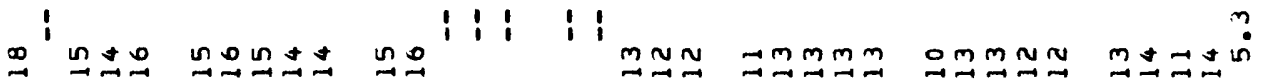

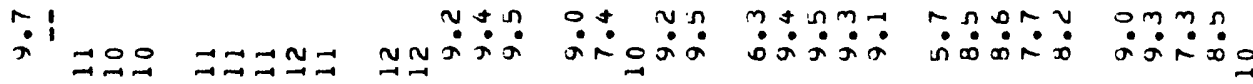


透的苍这

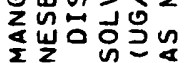

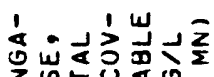
2

之的崖出

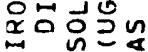

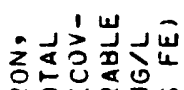

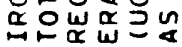

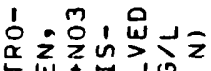

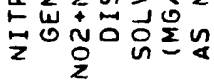

D

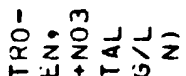

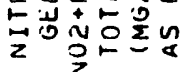

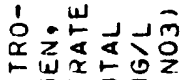

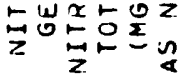

㩆的话

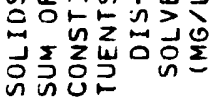

กันั้ำ

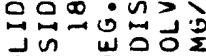

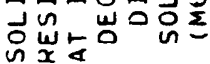

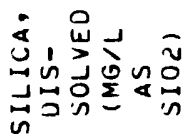

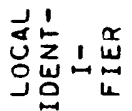

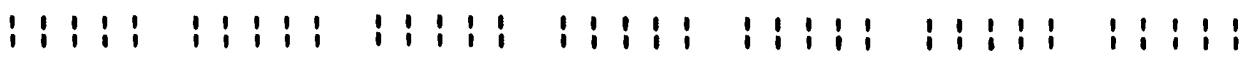

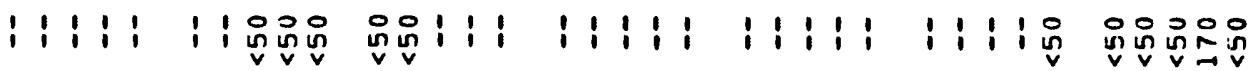

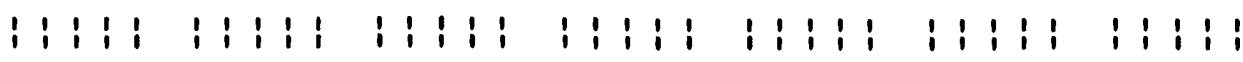

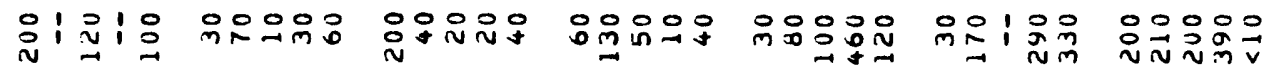

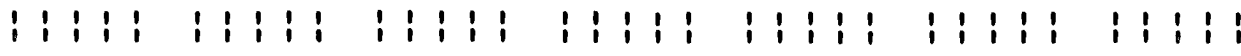

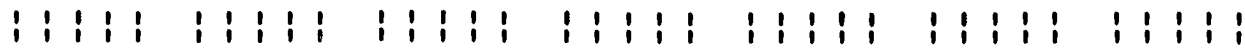

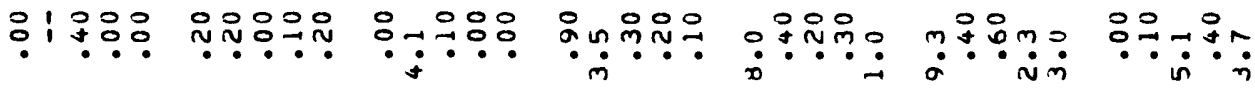

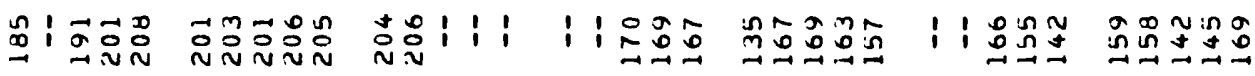

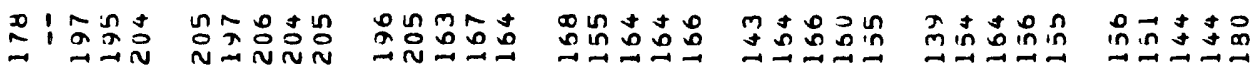
f

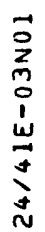




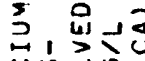

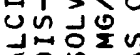

1

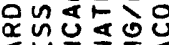

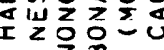

Luñ

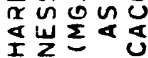

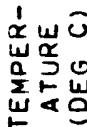

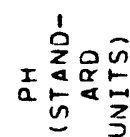

品它它蒙

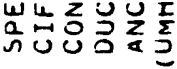

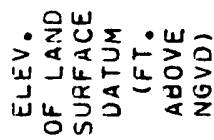

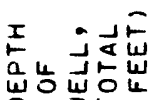

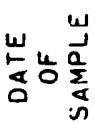

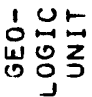

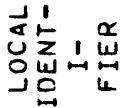

망은

$\vec{m} \pm m \pm$

구요

웅ํำ

ำกำ

तี゙N

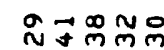

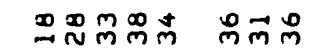

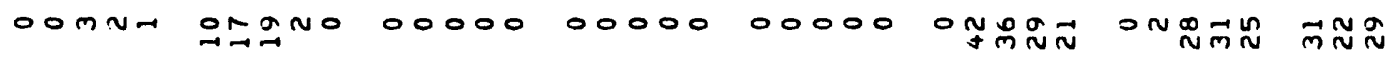

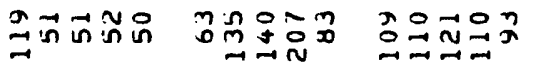

ぷロさ゚

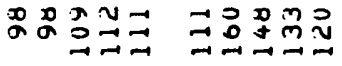

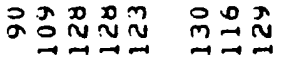

$\because 00$ 눙

$\dot{\rightarrow} \dot{\rightarrow} \dot{n} \dot{\sim} \dot{0}$

$\because \cong: x:$

in in 0 : n

$\because \because 9$ :

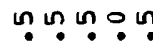

00000

ตำ แก ด ด ?

ำกำ

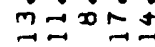

品品卞

$\dot{0} \dot{\dot{n}}$

$\simeq \dot{000}$

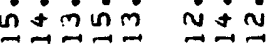

$+\sin \rightarrow \infty g$ or 0 in

ก.

$\sim \infty \infty \sim$

$\because 0+0 \infty$

$\because \because \because \because 0 \stackrel{0}{0}$

ratio risis

rinirín

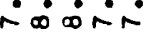

ínin

inimion

orris dir

คo $\rightarrow 0$

ำ

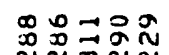

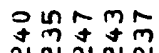

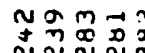

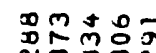

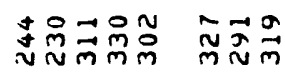

\section{1:응 \\ 正二五 \\ 吉志志}

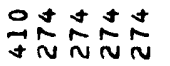

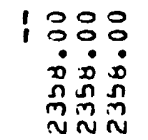

옹

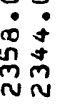

웅ำก

ㅇํำำ尔

กำ กำ

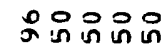

1영응 응응

荡官官宫

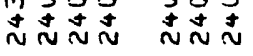

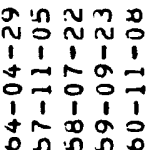

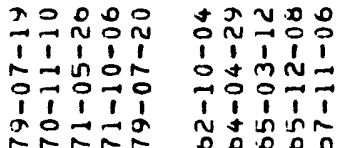

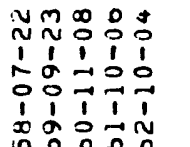

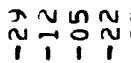

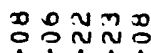

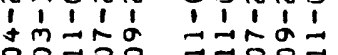

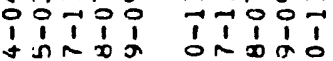

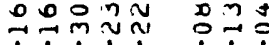

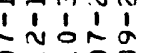

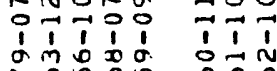

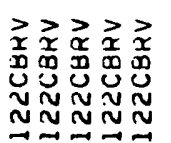

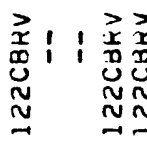

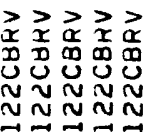

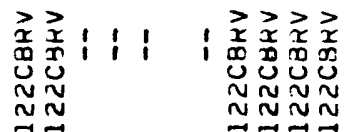

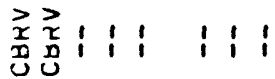

Uิ

$\rightarrow-$

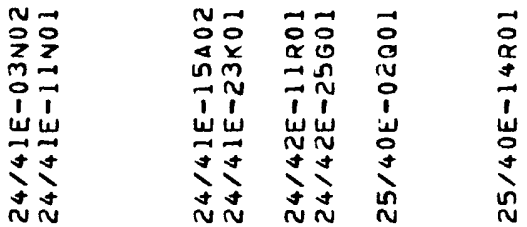

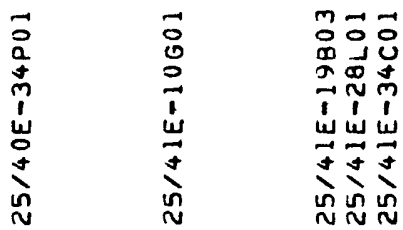




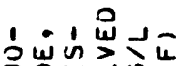

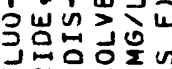

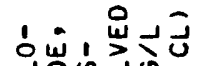

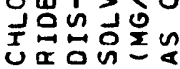

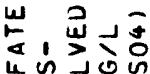

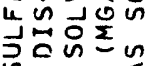

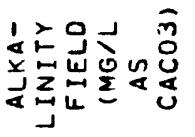

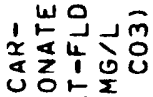
㟧

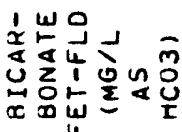

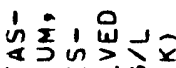

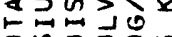

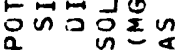

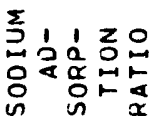

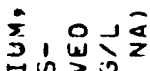

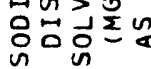

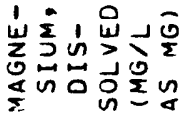

过志乐

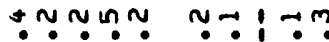

in in in in $m$

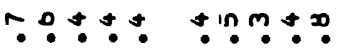

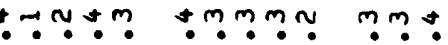

in $0 \mathrm{non}$

nก⿻ำ

$\because \because \because$

$\infty$ in

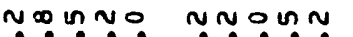

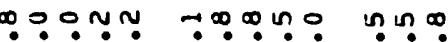
nann

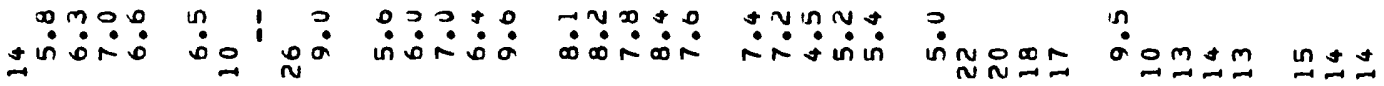
요용요

$\stackrel{\sim}{\sim} \cong \vec{n}:$

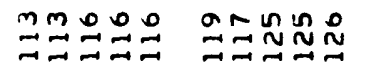

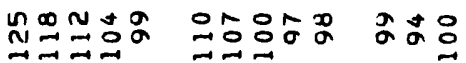

00000

00000

00000

ONO00 00000

0000000000000

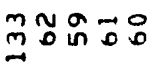

设卞出

$\ln _{\rightarrow \rightarrow \infty}^{\infty} \underset{n}{\infty}$

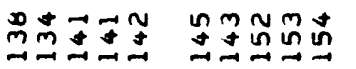

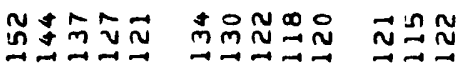

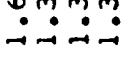

$0 ?: 9$

mo in un

ก: $: \div: \div$

บ?ตก:?

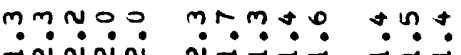
บm $m$

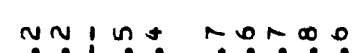

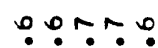

$\because: \div$

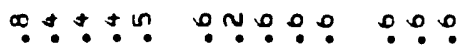
บ?อ?

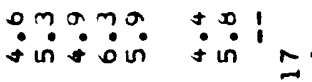

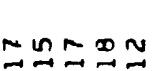

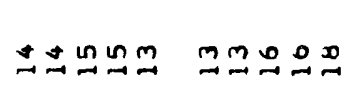

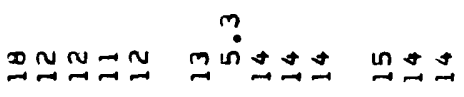
:ำ:

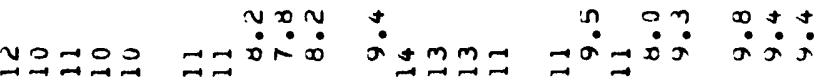

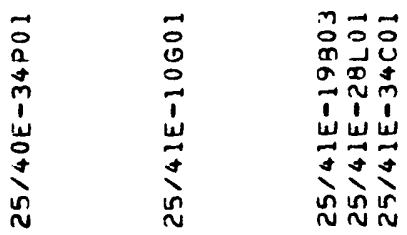




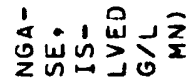

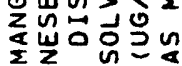

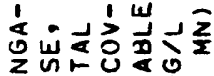

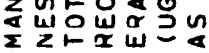

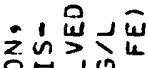
좀영워

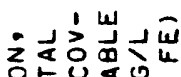

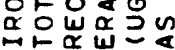

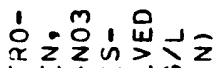

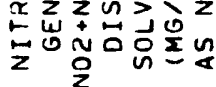

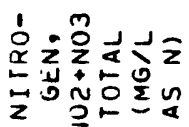

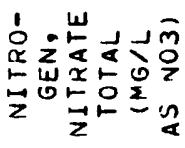
的的品三

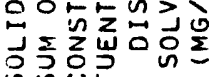

的纾。”岂

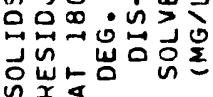

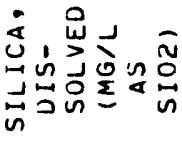

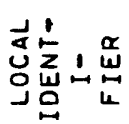

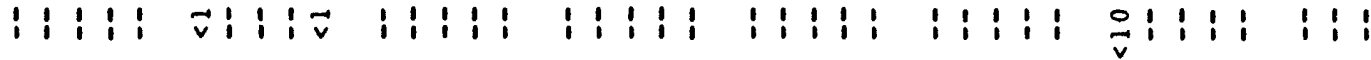

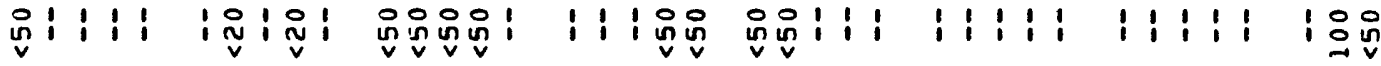

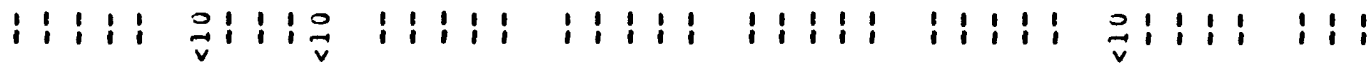

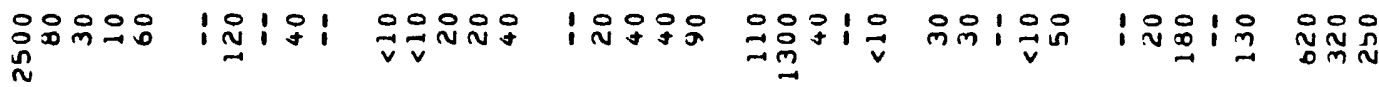

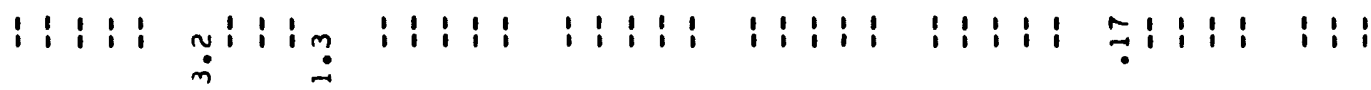

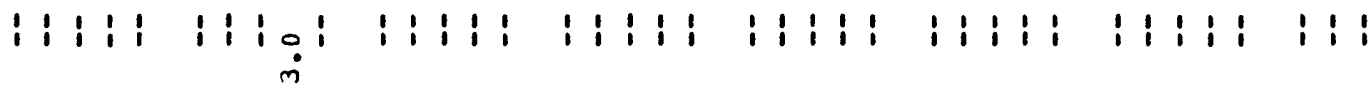

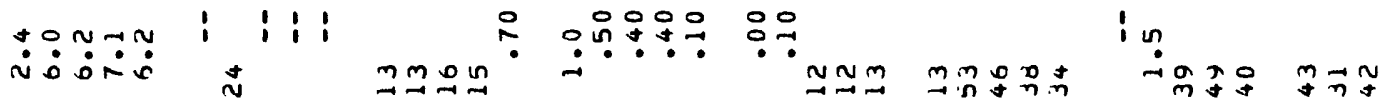

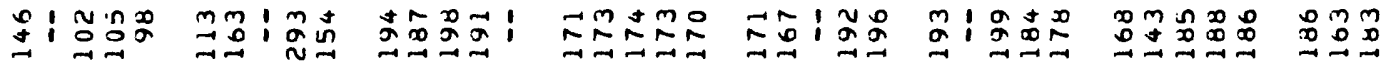

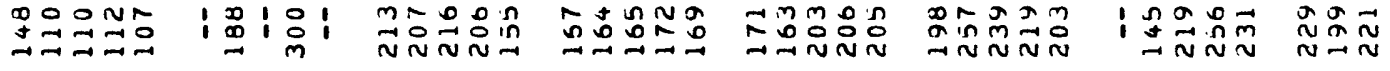

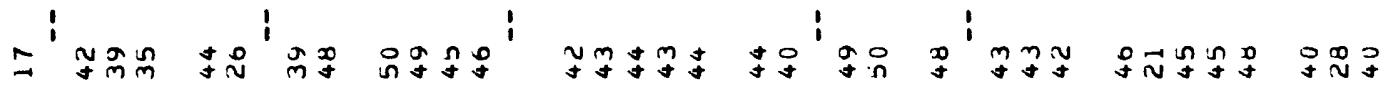

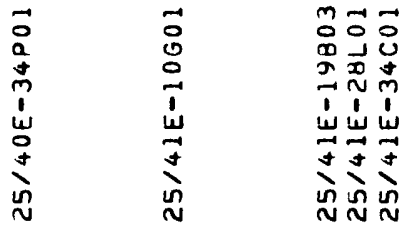


至, 品远

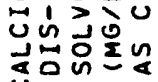

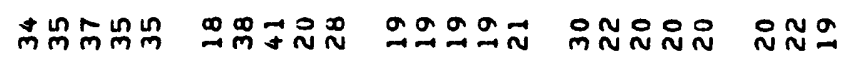

1. 문

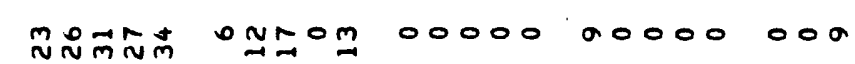

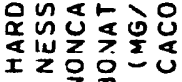

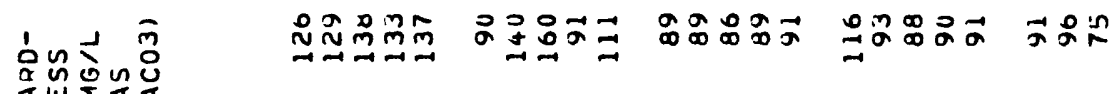

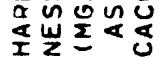

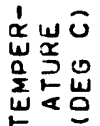

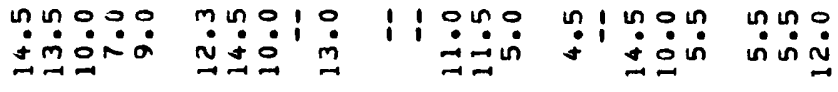

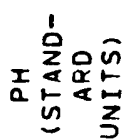

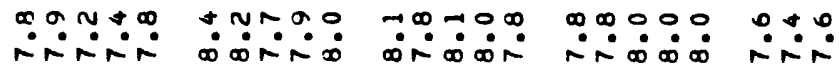

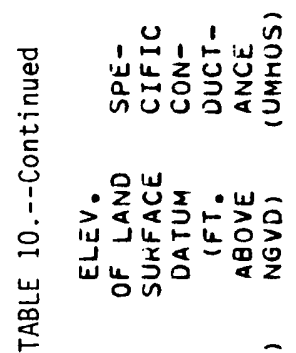

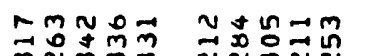

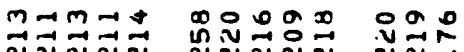

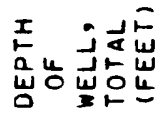

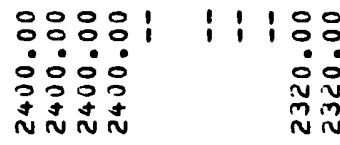

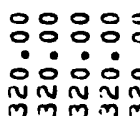

영영

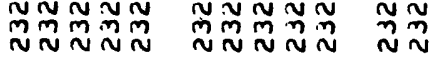

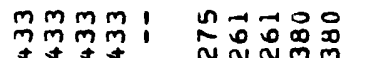

응용요요요

啹品品品 品品品

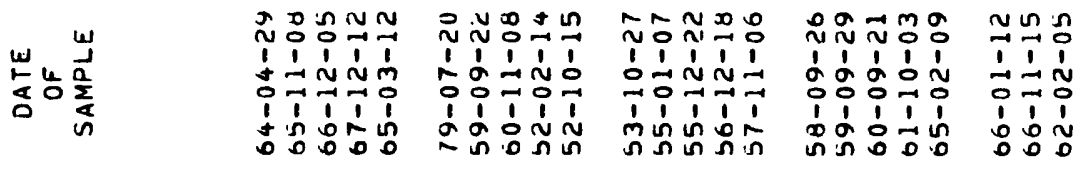

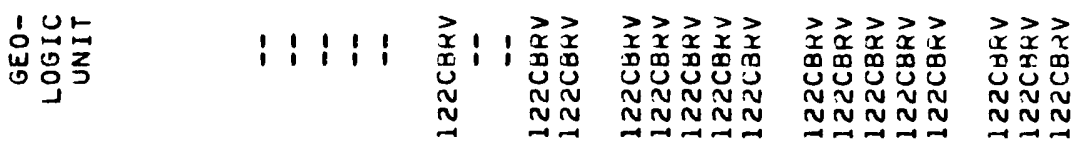

过支点点点

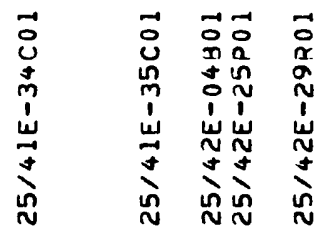

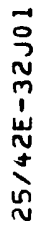




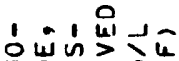

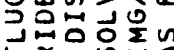

لَّد دُ

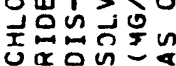

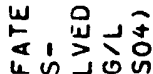

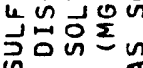

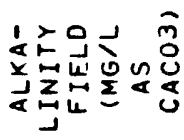

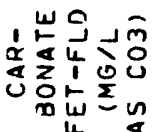

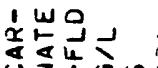

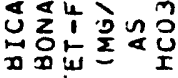

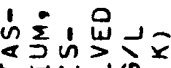

田牙的

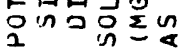

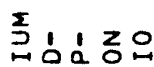

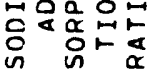

辛, 品文

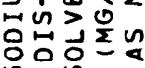

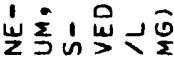

可的范定的

$\Sigma$

还占采岕

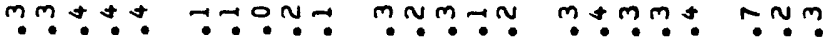

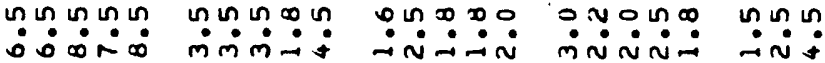

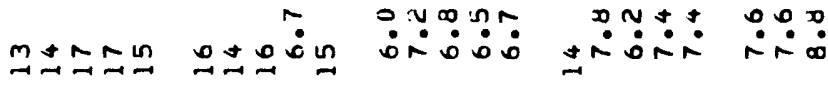

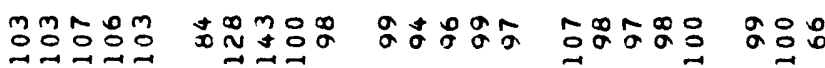

00000000000000000000000

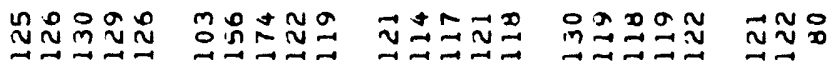

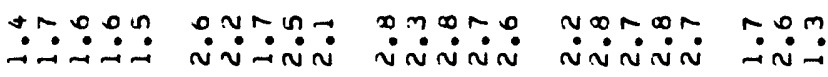

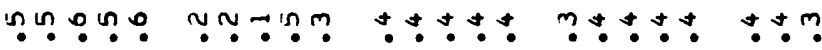

mants

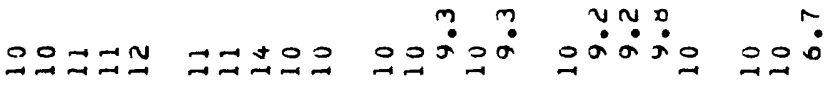

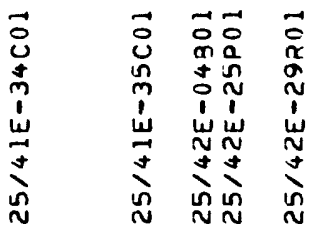

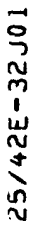




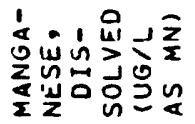

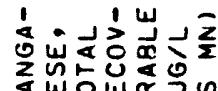

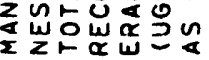

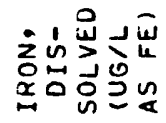
之ئ

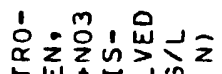

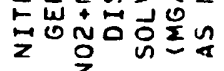

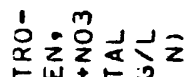

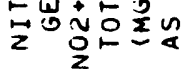

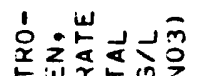

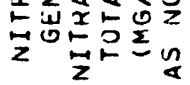

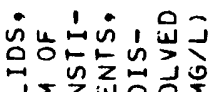

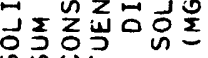

的。”

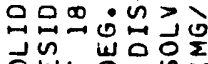

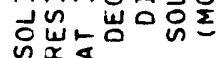

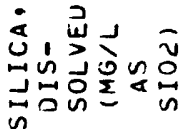

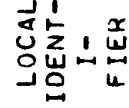

1:11:

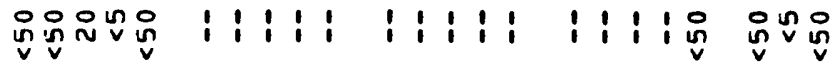
1:11:

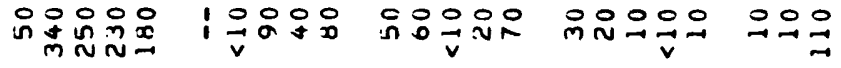
1919

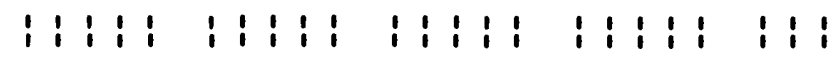

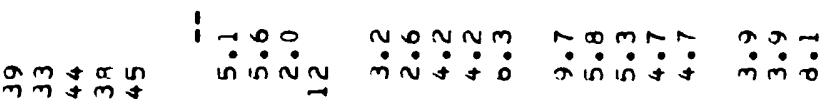

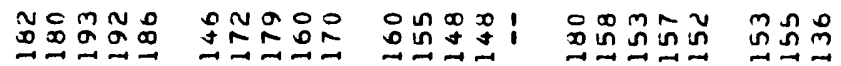

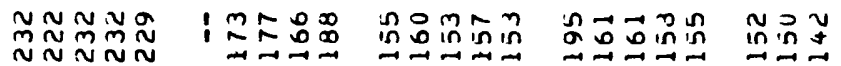

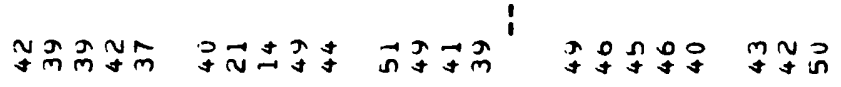


TABLE 11.--Surmary of physical and major shemical-constituent data for ground-water samples from the Airway Heights subregion

[Values in milligrams per liter except as indicated umho, micromho; col $/ 100 \mathrm{~mL}$, colonies per 100 milliliter; $\mathrm{ug} / \mathrm{L}$, microgram per liter]

\begin{tabular}{|c|c|c|c|c|}
\hline Constituent & $\begin{array}{l}\text { Jumber of } \\
\text { sample sites }\end{array}$ & $\frac{\text { Trace elem }}{\text { Maximum }}$ & $\frac{\text { nt concentr }}{\text { Minimum }}$ & $\frac{\text { rations }}{\text { Median }}$ \\
\hline Specific conductance (umho) & 21 & 458 & 121 & 260 \\
\hline $\mathrm{pH}$ (units) & 21 & 8.4 & 6.8 & 7.6 \\
\hline Temperature $\left({ }^{\circ} \mathrm{C}\right)$ & 21 & 21.0 & 4.5 & 12.6 \\
\hline Fecal-coliform bacteria (col/100 mL) & -- & -- & -- & -- \\
\hline Hardness (as $\mathrm{CaCO}_{3}$ ) & 21 & 210 & 50 & 109 \\
\hline Hardness, noncarbonate (as $\mathrm{CaCO}_{3}$ ) & 21 & 42 & 0 & 2 \\
\hline Calcium, dissolved & 21 & 58 & 13 & 28 \\
\hline Magnesium, dissolved & 21 & 15 & 4 & 9.9 \\
\hline Sodium, dissolved & 21 & 18 & 3.1 & 11.8 \\
\hline Sodium-adsorption ratio & 21 & 2.2 & .1 & .5 \\
\hline Potassium, dissolved & 21 & 5.1 & 1.1 & 1.7 \\
\hline Bicarbonate & 21 & 250 & 59 & 132 \\
\hline Carbonate & 21 & 2 & 0 & 0 \\
\hline Alkalinity (as $\mathrm{CaCO}_{3}$ ) & 21 & 205 & 48 & 108 \\
\hline Sulfate, dissolved & 21 & 26 & 4.5 & 10 \\
\hline Chloride, dissolved & 21 & 10 & 1 & 3.1 \\
\hline Fluoride, dissolved & 21 & .8 & .0 & .3 \\
\hline Silica, dissolved (as $\mathrm{SiO}_{2}$ ) & 21 & 55 & 14 & 43 \\
\hline Solids, dissolved (residue at $180^{\circ} \mathrm{C}$ ) & 17 & 300 & 107 & 178 \\
\hline Solids, dissolved (sum of constituents) & 21 & 306 & 105 & 172 \\
\hline Nitrate (as N) & 21 & 12 & .00 & 1.3 \\
\hline Iron, total recoverable $(u g / L)$ & 17 & 2,500 & 0 & 73 \\
\hline Iron, dissolved $(\mathrm{ug} / \mathrm{L})$ & 4 & 10 & 0 & 0 \\
\hline Manganese, total recoverable (ug/L) & 11 & 170 & 5 & $<50$ \\
\hline Manganese, dissolved (ug/L) & 4 & 10 & 1 & 1 \\
\hline
\end{tabular}


TABLE 12.--Trace-element concentrations in ground-water samples from the Airway Heights subregion

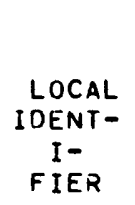

$24 / 41 E-15 A 02$

$24 / 41 E-23 K O 1$

$24 / 42 E-11 R 01$

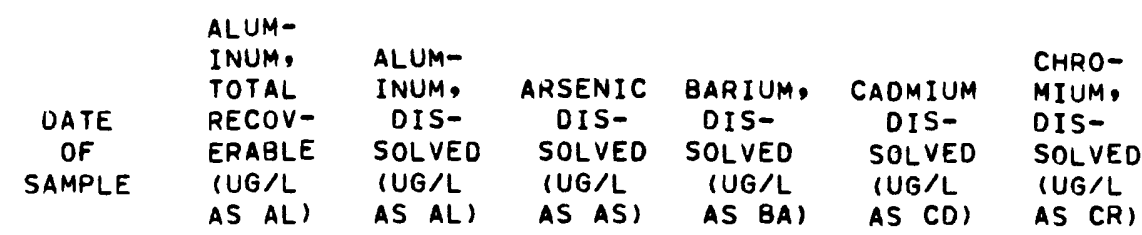

Spokane County

$\begin{array}{llllllr}79-07-19 & -- & 0 & 2 & 20 & <1 & 0 \\ 70-11-10 & 10 & -- & -- & -- & -- & <30 \\ 71-10-06 & 10 & -- & -- & -- & -- & <30\end{array}$

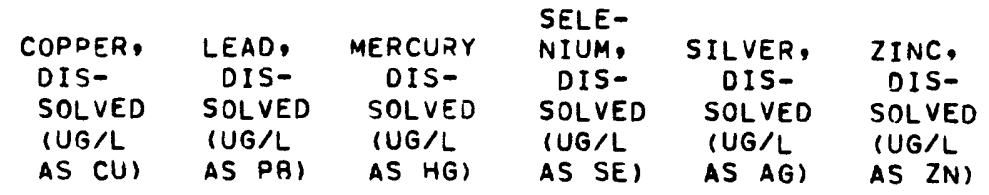

$\begin{array}{rrrrrr}1 & 0 & 1.9 & 0 & 0 & 110 \\ <50 & <100 & -- & -- & -- & 200 \\ <50 & <100 & -- & -- & -- & 70\end{array}$


TABLE 13.--Physical, biological, and major chemical-constituent data for ground-water samples from the Chamokane Creek subregion

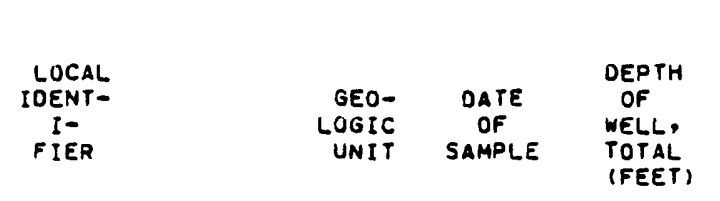

ELEV.

OF LAND

SURFACE

IFT.

ABOVE

NGVD)

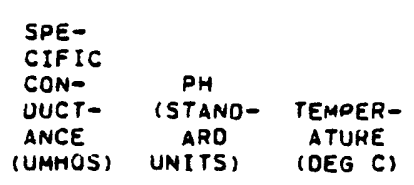

COLI-

FORM,

HARO-

0.7

UM-MF

(COLS.)

NESS

IUG/L

AS

(ACO3)

NESS.

NONCAR-

BONATE

(MG/L

Stevens County
$27 / 39 E-13 F 01$

$28 / 39 E-24 G 01$

28/39E-25HOL

28/40E-05001

$28 / 40 E-17 L O I$

$29 / 40 E-22001$
$28 / 39 \varepsilon-35 L 01$

$\begin{array}{ll}\text { 11OALVM } & 79-07-25 \\ \text { 11OALVM } & 79-07-25 \\ -- & 59-12-01 \\ -- & 60-05-17 \\ 110 A L V M & 79-07-25 \\ \text { 11OALVM } & 79-07-25 \\ 110 A L V M & 79-07-25 \\ \text { 11OALVM } 79-07-25\end{array}$

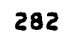

$\begin{array}{rrr}282 & -2 & 390 \\ 42 & -0 & 412 \\ 350 & 1771.00 & 271 \\ 350 & 1771.00 & 279\end{array}$

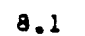

8.1
7.6
7.8

7.8
8.0

$131 \quad 1700.00 \quad 355$

7.9

$\begin{array}{ll}-- & 339 \\ -- & 325\end{array}$

7.6
7.8
7.8

60
41
61

397
17.1
11.5
15.5
12.3

$\begin{array}{ccc}\text { SOOIUM } & \text { POTAS- } & \text { BICAR- } \\ \text { AD- } & \text { SIUM- } & \text { BONATE } \\ \text { SORP- } & \text { OIS- } & \text { FET-FLO } \\ \text { TION } & \text { SOLVEO } & \text { (MG/L } \\ \text { RATIO } & \text { (MG/L } & \text { AS } \\ & \text { AS K) } & \text { HCO3) }\end{array}$

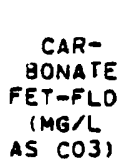

ALKA-

LINITY

FIELO

IMGLL

AS

CACO3)

$\begin{array}{ll}<1 & 197 \\ <1 & 210 \\ \therefore- & 129 \\ <- & 132 \\ <1 & 165 \\ & \\ <1 & 165 \\ <1 & 154 \\ <1 & 197\end{array}$

(MG/L

\begin{tabular}{|c|c|c|}
\hline $\begin{array}{l}\text { CALCIUM } \\
\text { UIS- } \\
\text { SOLVED } \\
\text { IMG/L }\end{array}$ & $\begin{array}{l}\text { MAGNE- } \\
\text { SIUM, } \\
\text { DIS- } \\
\text { SOLVEO } \\
\text { IMG/L }\end{array}$ & $\begin{array}{l}\text { SUOIUM, } \\
\text { OIS- } \\
\text { SOLVEO } \\
\text { IMG/L }\end{array}$ \\
\hline AS CAI & AS MGI & AS NAI \\
\hline
\end{tabular}

\begin{tabular}{|c|c|c|c|c|c|}
\hline $\begin{array}{l}36 \\
48 \\
36\end{array}$ & $\begin{array}{l}26 \\
22 \\
9.5\end{array}$ & $\begin{array}{l}4.3 \\
5.1 \\
7.3\end{array}$ & $\begin{array}{l}.1 \\
.2 \\
.3\end{array}$ & $\begin{array}{l}1.2 \\
2.8 \\
2.2\end{array}$ & $\begin{array}{l}232 \\
256 \\
173\end{array}$ \\
\hline $43^{--}$ & $16^{--}$ & $10^{--}$ &.-4 & 3.0 & $\begin{array}{l}176 \\
207\end{array}$ \\
\hline $\begin{array}{l}38 \\
37 \\
46\end{array}$ & $\begin{array}{l}17 \\
15 \\
20\end{array}$ & $\begin{array}{l}5.0 \\
6.0\end{array}$ & $\begin{array}{r}.2 \\
.2\end{array}$ & $\begin{array}{l}2.6 \\
2.4\end{array}$ & $\begin{array}{l}195 \\
183 \\
244\end{array}$ \\
\hline
\end{tabular}

$\begin{array}{ll}\text { FLUO- } & \text { SILICA, } \\ \text { HIDE, } & \text { OIS- } \\ \text { OIS- } & \text { SOLVEO } \\ \text { SOLVEO } & \text { (MGLL } \\ \text { (MGNL } & \text { AS } \\ \text { AD F) } & \text { SIOZ) }\end{array}$

SOLIOS, SOLIOS, RESIOUE SUM OF AT 180 CONSTIOEG. C TUENTS, OIS-
SOLVED SOLVED

AD F) SIO2) (MG/L) (MG/L) AS NO3)

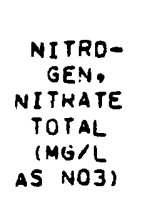

NITRO-

TEN,

$\mathrm{NO2}+\mathrm{NO}_{3}$

SOLVEO

(MG/L

AS $N$ )

IRON,
TOTAL
RECOV-
ERABLE
(UG/L
AS FE)

190
210
142
144
170

20

$9.7 \quad 1.5$

$5.9 \quad 1.2$

11

RIDE,

DIS- OIS-

SOLVED SOLVED

(MG/L

160
150
200

7.8
6.7
7.5

1.2

$7.5 \quad 1.3$

\begin{tabular}{|c|c|c|c|c|c|c|c|c|}
\hline 1 & $\begin{array}{l}13 \\
24\end{array}$ & $\ddot{-}$ & $\begin{array}{l}220 \\
239\end{array}$ & 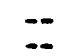 & $\begin{array}{l}.81 \\
.64\end{array}$ & $=$ & $\begin{array}{l}360 \\
100\end{array}$ & $\begin{array}{r}2 \\
<1\end{array}$ \\
\hline .5 & 24 & 165 & 172 & .00 & -- & 10 & $\cdots$ & 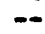 \\
\hline$-\overline{3}$ & $2^{--}$ & $\overline{-}$ & $=$ & $\because$ & $\overline{7}$ & $-\infty$ & $\overline{-}$ & $\ddot{-}$ \\
\hline & .2 & & 201 & 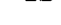 & os & 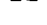 & 350 & \\
\hline .1 & $\begin{array}{l}27 \\
27\end{array}$ & $=$ & $\begin{array}{l}195 \\
106\end{array}$ & $=$ & .90 & $\because$ & 150 & $1^{2}$ \\
\hline & 31 & - & 235 & - & .78 & -. & 290 & 2 \\
\hline
\end{tabular}


TABLE 14.--Summary of physical, biological, and major chemical-constituent data for ground-water samples from the Chamokane Creek subregion

[Values in milligrams per liter except as indicated umho, micromho; col $/ 100 \mathrm{~mL}$, colonies per $100 \mathrm{milliliter}$; $u g / L$, microgram per liter]

\begin{tabular}{|c|c|c|c|c|}
\hline Constituent & $\begin{array}{l}\text { Number of } \\
\text { sample sites }\end{array}$ & $\begin{array}{l}\text { Trace ele } \\
\text { Maximum }\end{array}$ & $\begin{array}{l}\text { ent concen } \\
\text { Minimum }\end{array}$ & $\begin{array}{l}\text { tration } \\
\text { Medfan } \\
\end{array}$ \\
\hline Specific conductance (umho) & 7 & 412 & 271 & 355 \\
\hline $\mathrm{pH}$ (units) & 7 & 8.1 & 7.6 & 7.8 \\
\hline Temperature $\left({ }^{\circ} \mathrm{C}\right)$ & 7 & 17.1 & 10.3 & 12.0 \\
\hline Fecal-coliform bacteria (col /100 mL) & 6 & $<1$ & $<1$ & $<1$ \\
\hline Hardness (as $\mathrm{CaCO}_{3}$ ) & 7 & 210 & 130 & 170 \\
\hline Hardness, noncarbonate (as $\mathrm{CaCO}_{3}$ ) & 7 & 10 & 0 & 0 \\
\hline Calcium, dissolved & 7 & 48 & 36 & 38 \\
\hline Magnesium, dissolved & 7 & 26 & 9.5 & 17 \\
\hline Sodium, dissolved & 7 & 10 & 4.3 & 5.4 \\
\hline Sodium-adsorption ratio & 7 & .3 & .1 & .1 \\
\hline Potassium, dissolved & 7 & 4.2 & 2.2 & 2.8 \\
\hline Bicarbonate & 7 & 256 & 173 & 207 \\
\hline Carbonate & 7 & 0 & 0 & 0 \\
\hline Alkalinity $\left(\right.$ as $\left.\mathrm{CaCO}_{3}\right)$ & 7 & 210 & 142 & 170 \\
\hline Sulfate, dissolved & 7 & 20 & 5.9 & 7.8 \\
\hline Chloride, dissolved & 7 & 1.5 & .8 & 1.2 \\
\hline Fluoride, dissolved & 7 & .5 & .1 & .1 \\
\hline Silica, dissolved (as $\mathrm{SiO}_{2}$ ) & 7 & 31 & 13 & 24 \\
\hline Solids, dissolved (residue at $180^{\circ} \mathrm{C}$ ) & 1 & 165 & 165 & 165 \\
\hline Solids, dissolved (sum of constituents) & 7 & 242 & 172 & 207 \\
\hline Nitrate (as N) & 7 & 1.1 & .00 & .78 \\
\hline Iron, total recoverable (ug/L) & 1 & 10 & 10 & 10 \\
\hline Iron, dissolved (ug/L) & 6 & 360 & 100 & 220 \\
\hline Manganese, total recoverable $(4 \mathrm{~g} / \mathrm{L})$ & -- & -- & -- & -- \\
\hline Manganese, dissolved (ug/L) & 6 & 90 & $<1$ & 2 \\
\hline
\end{tabular}


TABLE 15.--Trace-element concentrations in ground-water samples from the Chamokane Creek subregion

$$
\begin{aligned}
& \text { LOCAL } \\
& \text { IDENT- } \\
& \text { I- } \\
& \text { FIER }
\end{aligned}
$$

$28 / 39 E-35 L 01$

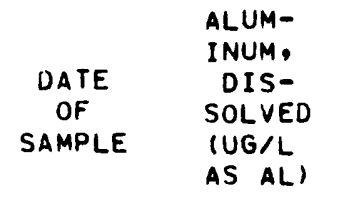

ARSENIC BARIUM, DIS- DISSOLVED SOLVED IUG/L IUG/L AS AS ) AS BA)

$\underline{\text { Stevens County }}$

MIUM,

DIS-

SOLVED

(UG/L

AS (R)

SOLVED

IUG/L

AS (D)

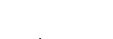

COPPER, DIS-

SOLVED

IUG/L

AS CU)

\begin{abstract}
$79-07-25$
\end{abstract}
10

9

80

$<1$

10

4
0

.1

0

0

110 
TABLE 16.--Physical, biological, and major chemical-constituent data for ground-water samples from the Curlew-Sanpoil subregion

\begin{tabular}{|c|c|c|c|c|c|c|c|c|c|c|}
\hline $\begin{array}{l}\text { LOCAL } \\
\text { SOENT- } \\
\text { I- } \\
\text { FIER }\end{array}$ & $\begin{array}{l}\text { GEO- } \\
\text { LOGIC } \\
\text { UNIT }\end{array}$ & $\begin{array}{c}\text { OATE } \\
\text { OF } \\
\text { SAMPLE }\end{array}$ & $\begin{array}{l}\text { DEPTH } \\
\text { OF } \\
\text { WELL. } \\
\text { TOTAL } \\
\text { (FEET) }\end{array}$ & $\begin{array}{l}\text { ELEV. } \\
\text { OF LANO } \\
\text { SURFACE } \\
\text { OATUM } \\
\text { IFT. } \\
\text { NGVOI }\end{array}$ & $\begin{array}{l}\text { SPE- } \\
\text { CIFIC } \\
\text { CON- } \\
\text { OUCT- } \\
\text { ANCE } \\
\text { (MICRO- } \\
\text { MHOS) }\end{array}$ & $\begin{array}{c}\text { PH } \\
\text { FIELD } \\
\text { (UNITS) }\end{array}$ & $\begin{array}{c}\text { TEMPER- } \\
\text { ATURE, } \\
\text { WATER } \\
\text { (DEG C) }\end{array}$ & $\begin{array}{l}\text { COLI- } \\
\text { FORM, } \\
\text { FECAL, } \\
0.7 \\
\text { UM-MF } \\
\text { (COLS.' } \\
100 \text { YL) }\end{array}$ & $\begin{array}{l}\text { HARO- } \\
\text { NESS } \\
\text { (MG/L } \\
\text { AS } \\
\text { CACO3) }\end{array}$ & $\begin{array}{l}\text { HARO- } \\
\text { NESS } \\
\text { NONCAR- } \\
\text { BONATE } \\
\text { (MG/L } \\
\text { CACD3) }\end{array}$ \\
\hline
\end{tabular}

\section{Ferry County}

\begin{tabular}{|c|c|c|c|c|c|c|c|c|c|c|}
\hline $\begin{array}{l}29 / 33 E-04 M 01 \\
32 / 33 E-32 N O 1 \\
36 / 33 E-07 F 01\end{array}$ & $\begin{array}{l}110 A L V M \\
110 A L V M \\
110 A L V M \\
112 G L C V \\
112 G L C V\end{array}$ & $\begin{array}{l}67-10-17 \\
79-06-25 \\
79-06-25 \\
70-11-12 \\
71-05-27\end{array}$ & $\begin{array}{r}205 \\
205 \\
18 \\
80 \\
80\end{array}$ & $\begin{array}{l}1304.00 \\
1304.00 \\
1640.00 \\
2270.00 \\
2270.00\end{array}$ & $\begin{array}{l}286 \\
242 \\
225 \\
444 \\
318\end{array}$ & $\begin{array}{l}7.8 \\
7.4 \\
7.0 \\
7.2 \\
7.6\end{array}$ & $\begin{array}{l}10.6 \\
11.8 \\
9.4 \\
7.2 \\
5.8\end{array}$ & $\begin{array}{l}-\infty \\
<1 \\
<1 \\
-\infty\end{array}$ & $\begin{array}{r}131 \\
120 \\
98 \\
188 \\
130\end{array}$ & $\begin{array}{r}3 \\
0 \\
9 \\
66 \\
30\end{array}$ \\
\hline $\begin{array}{l}36 / 33 E-07 F 02 \\
37 / 33 E-08 \cup 01 \\
38 / 33 E-11 N O 1\end{array}$ & $\begin{array}{l}112 G L C V \\
112 G L C V \\
112 G L C V \\
112 G L C V \\
112 G L C V\end{array}$ & $\begin{array}{l}79-06-25 \\
60-04-07 \\
60-10-20 \\
79-06-24 \\
79-06-23\end{array}$ & $\begin{array}{l}80 \\
79 \\
79 \\
97 \\
63\end{array}$ & $\begin{array}{l}2270.00 \\
2270.00 \\
2270.00 \\
2330.00 \\
2200.00\end{array}$ & $\begin{array}{l}355 \\
375 \\
394 \\
490 \\
285\end{array}$ & $\begin{array}{l}7.3 \\
7.0 \\
7.2 \\
7.6 \\
7.6\end{array}$ & $\begin{array}{r}8.2 \\
-.0 \\
7.0 \\
10.8 \\
8.4\end{array}$ & $\begin{array}{l}<1 \\
-\infty \\
<1 \\
<1\end{array}$ & $\begin{array}{l}140 \\
150 \\
167 \\
220 \\
120\end{array}$ & $\begin{array}{r}30 \\
43 \\
59 \\
20 \\
0\end{array}$ \\
\hline \multirow[t]{2}{*}{$39 / 33 E-01 J 01$} & $110 A L V M$ & $79-06-24$ & 36 & 1750.00 & 135 & 7.1 & 10.4 & - & - & \\
\hline & $\begin{array}{l}\text { CALCIUM } \\
\text { DIS- } \\
\text { SOLVED } \\
\text { (MG/L } \\
\text { AS CA) }\end{array}$ & $\begin{array}{l}\text { MAGNE- } \\
\text { SIUM. } \\
\text { DIS- } \\
\text { SOLVEO } \\
\text { (MG/L } \\
\text { AS MG) }\end{array}$ & $\begin{array}{l}\text { SOOIUM, } \\
\text { OIS- } \\
\text { SOLVED } \\
\text { (MG/L } \\
\text { AS NA) }\end{array}$ & $\begin{array}{l}\text { SOOIUM } \\
\text { AO- } \\
\text { SORP- } \\
\text { TION } \\
\text { RATIO }\end{array}$ & $\begin{array}{l}\text { POTAS- } \\
\text { SIUM, } \\
\text { DIS- } \\
\text { SOLVEO } \\
\text { (ME/L } \\
\text { AS K) }\end{array}$ & $\begin{array}{c}\text { BICAR- } \\
\text { BONATE } \\
\text { (MG/L } \\
\text { AS } \\
\text { HCO3) }\end{array}$ & $\begin{array}{l}\text { CAR- } \\
\text { BONATE } \\
\text { (MG/L } \\
\text { AS CO3) }\end{array}$ & $\begin{array}{l}\text { ALKA- } \\
\text { LINITY } \\
\text { IMG/L } \\
\text { AS } \\
\text { CACO3) }\end{array}$ & $\begin{array}{l}\text { SULFATE } \\
\text { OIS- } \\
\text { SOLVEO } \\
\text { (MG/L } \\
\text { AS SO4) }\end{array}$ & $\begin{array}{l}\text { CHLO- } \\
\text { RIDE, } \\
\text { OIS- } \\
\text { SOLVEO } \\
\text { (MG/L } \\
\text { AS CL) }\end{array}$ \\
\hline
\end{tabular}

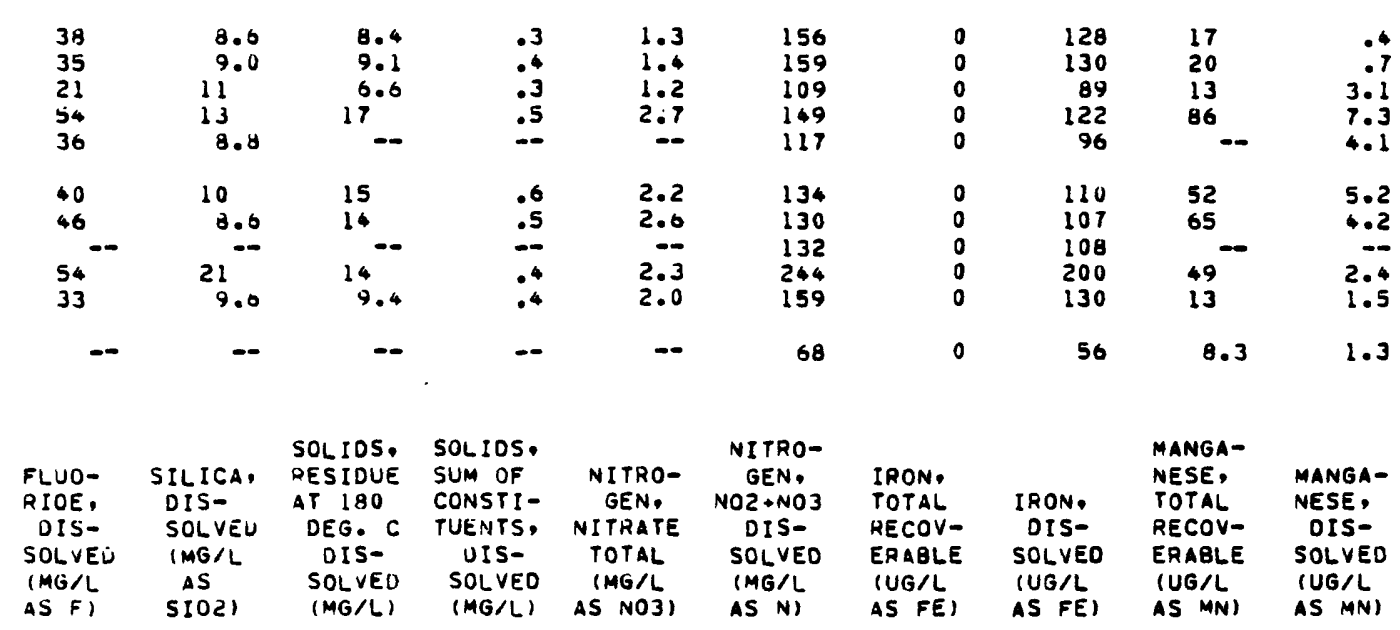

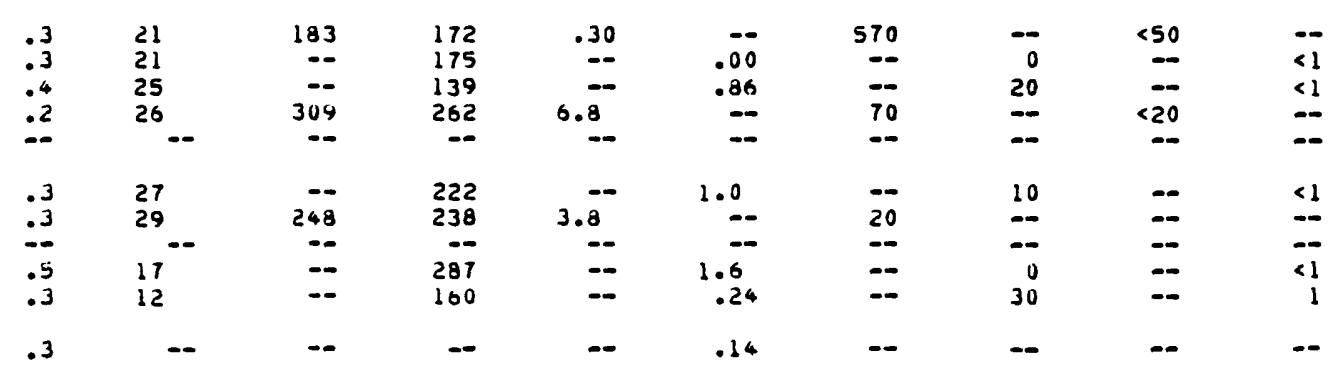


TABLE 17.--Summary of physical, biological, and major chemical-constituent data for ground-water samples from the Curlew-Sanpoil subregion

[Values in milligrams per 1 iter except as indicated umho, micromho; col $/ 100 \mathrm{~mL}$, colonies per 100 milliliter; $u g / L$, microgram per liter]

\begin{tabular}{|c|c|c|c|c|}
\hline Consti tuent & $\begin{array}{c}\text { Number of } \\
\text { sample sites }\end{array}$ & $\frac{\text { Trace elen }}{\text { Maximum }}$ & $\frac{\text { ent concen }}{\text { Minimum }}$ & $\frac{\text { itration }}{\text { Median }}$ \\
\hline Specific conductance (umho) & 7 & 490 & 135 & 289 \\
\hline $\mathrm{pH}$ (units) & 7 & 7.8 & 7.0 & 7.4 \\
\hline Temperature $\left({ }^{\circ} \mathrm{C}\right)$ & 7 & 11.8 & 5.8 & 9.4 \\
\hline Fecal-coliform bacteria (col/100 mL) & 5 & $<1$ & $<1$ & $<1$ \\
\hline Hardness (as $\mathrm{CaCO}_{3}$ ) & 6 & 220 & 98 & 139 \\
\hline Hardness, noncarbonate (as $\mathrm{CaCO}_{3}$ ) & 6 & 66 & 0 & 14.5 \\
\hline Calcium, dissolved & 6 & 54 & 21 & 40 \\
\hline Magnesium, dissolved & 6 & 21 & 8.6 & 10.1 \\
\hline Sodium, dissolved & 6 & 17 & 6.6 & 11.7 \\
\hline Sodium-adsorption ratio & 6 & .6 & .3 & .4 \\
\hline Potassium, dissolved & 6 & 2.7 & 1.2 & 2.2 \\
\hline Bicarbonate & 7 & 244 & 68 & 133 \\
\hline Carbonate & 7 & 0 & 0 & 0 \\
\hline Alkalinity (as $\mathrm{CaCO}_{3}$ ) & 7 & 200 & 56 & 109 \\
\hline Sulfate, dissolved & 7 & 86 & 8.3 & 18.5 \\
\hline Chloride, dissolved & 7 & 7.3 & .4 & 2.4 \\
\hline Fluoride, dissolved & 7 & .5 & .2 & .3 \\
\hline Silica, dissolved (as $\mathrm{SiO}_{2}$ ) & 6 & 29 & 12 & 23 \\
\hline Solids, dissolved (residue at $180^{\circ} \mathrm{C}$ ) & 3 & 309 & 183 & 248 \\
\hline Solids, dissolved (sum of constituents) & 6 & 287 & 139 & 206 \\
\hline Nitrate (as $\mathrm{N}$ ) & 7 & 1.6 & .04 & .86 \\
\hline Iron, total recoverable (ug/L) & 3 & 570 & 20 & 70 \\
\hline Iron, dissolved $(u g / L)$ & 5 & 30 & 0 & 10 \\
\hline Manganese, total recoverable (ug/L) & 2 & $<50$ & $<20$ & -- \\
\hline Manganese, dissolved (ug/L) & 5 & 1 & $<1$ & $<1$ \\
\hline
\end{tabular}


TABLE 18.--Trace-element concentrations in ground-water samples from the Curlew-Sanpoil subregion

\begin{tabular}{|c|c|c|c|c|c|c|c|}
\hline $\begin{array}{l}\text { LOCAL } \\
\text { IDENT- } \\
\text { I- } \\
\text { FIER }\end{array}$ & $\begin{array}{c}\text { UATE } \\
\text { OF } \\
\text { SAMPLE }\end{array}$ & $\begin{array}{l}\text { ALUM- } \\
\text { INUM, } \\
\text { TOTAL } \\
\text { RECOV- } \\
\text { ERABLE } \\
\text { IUG/L }\end{array}$ & $\begin{array}{l}\text { ALUM- } \\
\text { INUM, } \\
\text { DIS- } \\
\text { SOLVED } \\
\text { IUG/L }\end{array}$ & $\begin{array}{c}\text { ARSENIC } \\
\text { DIS- } \\
\text { SOLVED } \\
\text { IUG/L }\end{array}$ & $\begin{array}{l}\text { BARIUM, } \\
\text { DIS- } \\
\text { SOLVED } \\
\text { (UG/L }\end{array}$ & $\begin{array}{l}\text { CADMIUM } \\
\text { DIS- } \\
\text { SOLVED } \\
\text { IUG/L }\end{array}$ & $\begin{array}{l}\text { CHRO- } \\
\text { MIUM, } \\
\text { DIS- } \\
\text { SOLVED } \\
\text { IUG/L }\end{array}$ \\
\hline & & AS AL) & $A S A L)$ & AS ASI & AS BA) & AS $(D)$ & AS $(R)$ \\
\hline
\end{tabular}

\section{FERRY COUNTY}

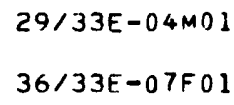

$$
\begin{aligned}
& 67-10-17 \\
& 79-06-25 \\
& 70-11-12 \\
& 79-06-25
\end{aligned}
$$

$\begin{array}{ll}-- & -- \\ -- & 10 \\ -- & --\end{array}$

$\begin{array}{rr}<10 & <1000 \\ 5 & 40 \\ -- & -- \\ 5 & 40\end{array}$

$<5$
1
--
$<1$

$<50$

0

COPDER, OIS-

SOLVED (UG/L AS CU)

LEAD,
DIS-
SOLVED
(UG/L
AS PB)

MERCURY
DIS-
SOLVEO
(UG/L
AS HG)

SELE -

NIUM,

DIS-

SOLVED

IUG/L

AS SE)

SILVER,

DIS-

SOLVED

IUG/L

AS AG)

DIS-

SOLVED

(UG/L

AS ZN)

$\begin{array}{rrrrrr}<400 & <40 & -- & <10 & <40 & <500 \\ 0 & 0 & -4 & 0 & 0 & 10 \\ <50 & <100 & -- & -- & -- & <10 \\ 4 & 0 & .5 & 2 & 0 & 20\end{array}$


TABLE 19.--Physical, biological, and major chemical-constituent data for ground-water samples from the Colville-Kettle subregion

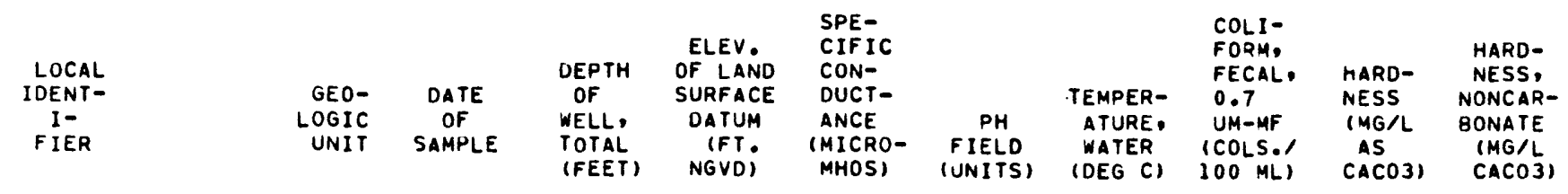

$37 / 37 E-08 A 02$

$37 / 37 E-08 R 02$

$32 / 40 E-28801$ $33 / 39 E-13 \mathrm{COI}$

$34 / 39 E-10 F 02$

$34 / 39 E-10 L 01$

$35 / 39 E-09 \mathrm{~J} 01$

$35 / 39 E-10 A 01$

$35 / 39 E-10 B 01$

$35 / 39 E-20 K 01$

$35 / 39 E-32 P 02$

$36 / 37 E-26001$

$36 / 37 E-26 R 01$

$36 / 37 E-35 A 01$

\section{ALVM 79-06-22 110 ALVM 79-06-23 \\ 64

$\begin{array}{cr}1340.00 & 408 \\ 1320.00 & 525 \\ \text { Stevens County }\end{array}$

-- 71-10-06

112 GLCV 79-06-21

$110 A L V M$ 79-06-21

110ALVM 61-05-02

$1126 L C V$ 71-10-06

112GLCV 60-01-25

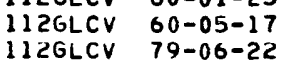

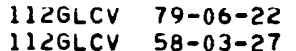

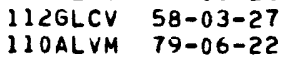

112 GLCV 79-06-22

$110 A L V M$ 67-09-26

$110 A L V M \quad 70-11-11$

$110 A L V M \quad 71-05-26$

$110 A L V M \quad 67-09-20$

110
520
48
38
90

236
236
236
210
110

290
58
67
67
72

208

280

300

303

427

1863.00

1900.00

1900.00

1900.00

1560.00

1880.00

1307.00

1325.00

1325.00

1300.00

635

298

403

418
327
$7.4 \quad 13.0$

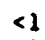

$<1$

180

10

13.6

$\begin{array}{ll}8.0 & 10.0 \\ 7.7 & 13.4 \\ 7.7 & 10.4 \\ 7.6 & 11.5 \\ 7.8 & 10.8\end{array}$

$-$

$<1$

$-$

130
130

147

200

7.8

$\begin{array}{ll}7.8 & 10.0 \\ 7.4 & 10.4\end{array}$

$\begin{array}{ll}7.4 & 10.4 \\ 7.7 & 10.0\end{array}$

-- 206

$\begin{array}{ll}-- & 206 \\ -1 & 210\end{array}$

$<1 \quad 200$

$\begin{array}{ll}-1 & 200 \\ <1 & 150\end{array}$

11.4

$7.4 \quad 12.4$

$\begin{array}{ll}<1 & 280 \\ -- & 145\end{array}$

$\begin{array}{rrrr}7.1 & 10.0 & -. & 145 \\ 7.9 & 8.4 & -- & 203\end{array}$

7.9

8.4

$\ddot{-}$

165

0
0
0
6
34
14
20
10
11
10

0
3
3
0
6

$$
\begin{aligned}
& \text { LOCAL } \\
& \text { IDENT- } \\
& \text { I- } \\
& \text { FIER }
\end{aligned}
$$

7/37E-0BAO2 $37 / 37 E-0 B R 02$

$32 / 40 E-28 B 01$ $33 / 39 E-13 C 01$ $34 / 39 E-10 F 02$ $34 / 39 E-10 L 01$ $35 / 39 E-09 J 01$ $35 / 39 E-10 A 01$

$35 / 39 E-10801$ $35 / 39 t-20 K 01$

$35 / 39 E-32 P 02$ $36 / 37 E-26001$ $36 / 37 E-26 R 01$

$36 / 37 E-35401$
MAGNE-

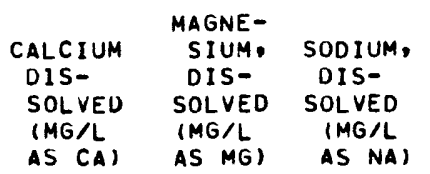

\begin{tabular}{|c|c|c|c|c|c|}
\hline \multirow[t]{2}{*}{$\begin{array}{l}49 \\
61\end{array}$} & $\begin{array}{l}15 \\
21\end{array}$ & $16^{6.1}$ &. .2 & $\begin{array}{l}1.0 \\
4.0\end{array}$ & $\begin{array}{l}207 \\
317\end{array}$ \\
\hline & & \multicolumn{4}{|c|}{ Stevens County } \\
\hline $\begin{array}{l}23 \\
33 \\
39 \\
46 \\
50\end{array}$ & $\begin{array}{l}9.4 \\
11 \\
8.4 \\
7.8 \\
19\end{array}$ & $\begin{array}{l}5.0 \\
5.6 \\
6.1 \\
6.2 \\
6.5\end{array}$ & $\begin{array}{r}.2 \\
.2 \\
.2 \\
.2 \\
.2\end{array}$ & $\begin{array}{l}1: 8 \\
1.5 \\
1: 8 \\
1: 7 \\
2.3\end{array}$ & $\begin{array}{l}129 \\
159 \\
171 \\
172 \\
206\end{array}$ \\
\hline $\begin{array}{l}60 \\
55 \\
57 \\
40\end{array}$ & $\begin{array}{l}14 \\
16^{--} \\
14 \\
11\end{array}$ & $\begin{array}{r}3.9 \\
-- \\
4.9 \\
3.6 \\
8.1\end{array}$ & $\begin{array}{r}.1 \\
-2 \\
.2 \\
.1 \\
.3\end{array}$ & $\begin{array}{r}1.4 \\
-.- \\
1.5 \\
2.0 \\
4.5\end{array}$ & $\begin{array}{l}234 \\
232 \\
232 \\
231 \\
171\end{array}$ \\
\hline $\begin{array}{l}86 \\
42 \\
55 \\
57 \\
46\end{array}$ & $\begin{array}{l}15 \\
9.6 \\
16 \\
17 \\
12\end{array}$ & $\begin{array}{r}16 \\
5.3 \\
6.5 \\
-5.4\end{array}$ & $\begin{array}{l}.4 \\
.2 \\
.2 \\
-.2 \\
.2\end{array}$ & $\begin{array}{r}3.8 \\
2.3 \\
3.8 \\
-0 \\
2.8\end{array}$ & $\begin{array}{l}354 \\
173 \\
244 \\
273 \\
194\end{array}$ \\
\hline
\end{tabular}

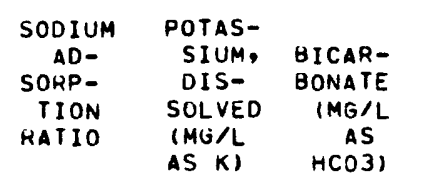

Ferry County

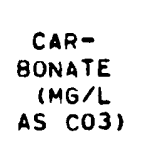

LIN
CHLO-

RIDE,

DIS-

SOLVED

$\begin{array}{ccc}A S & (M G / L & (M G / L \\ C A C O 3) & A S S O 4) & A S C L)\end{array}$ 
TABLE 19.---Continued

$$
\begin{aligned}
& \text { LOCAL } \\
& \text { IOENT- } \\
& \text { I- } \\
& \text { FIER }
\end{aligned}
$$

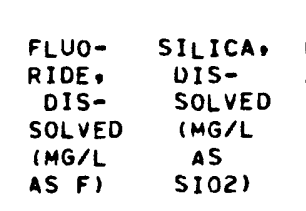

SOLIDS, RESIDUE AT 180 CONSTIDISSOLVED (MG/L)

SOLIDS,
SUM OF
CONSTI-
TUENTS,
DIS-
SOLVED
(MG/L)

NITRO- NITROGEN. GEN, NITRATE TOTAL (MG/L AS NO3)
GEN. $\mathrm{NO2}+\mathrm{NO3}$ TOTAL (MG/L AS N)
NITROGEN, $\mathrm{NO} 2+\mathrm{NO}_{3}$ DISSOLVED (MG/L AS NI
IRON, TOTAL RECOVERABLE IUG/L AS FE)

$\begin{array}{lll} & \text { MANGA- } \\ & \text { NESE, } & \text { MANGA- } \\ & \text { TOTAL } & \text { NESE: } \\ \text { IRON, } & \text { RECOV- } & \text { DIS- } \\ \text { OIS- } & \text { RECOV } & \text { SOLVE } \\ \text { SOLVED } & \text { ERABLE } & \text { SOLVED } \\ \text { (UG/L, (UG/L } & \text { (UG/L } \\ \text { AS FE) } & \text { AS MN) } & \text { AS MN) }\end{array}$

$37 / 37 E-08 A 02$ $37 / 37 E-08 R 02$

$32 / 40 E-28801$ $33 / 39 E-13 C 01$ $34 / 39 E-10 F O 2$

$34 / 39 E-1$ OLO 1

$35 / 39 E-09 J 01$

$35 / 39 E-10 A 01$

$35 / 39 E-10801$

$35 / 39 E-20 K 01$

$35 / 39 E-32 P 02$

36/37E-2600I

36/37E-26RO1

\begin{tabular}{|c|c|c|c|c|c|c|c|c|c|c|}
\hline .4 & $\begin{array}{l}17 \\
15\end{array}$ & $\because$ & $\begin{array}{l}230 \\
305\end{array}$ & $\because$ & $\ddot{--}$ & $\begin{array}{l}1.7 \\
.00\end{array}$ & $=$ & $\begin{array}{r}10 \\
0\end{array}$ & $\overline{-}$ & $<1$ \\
\hline \multicolumn{11}{|c|}{ Stevens County } \\
\hline $\begin{array}{l}.0 \\
.1 \\
.2 \\
.2 \\
.0\end{array}$ & $\begin{array}{l}31 \\
16 \\
21 \\
23 \\
18\end{array}$ & $\begin{array}{r}150 \\
-- \\
-- \\
202 \\
284\end{array}$ & $\begin{array}{l}144 \\
103 \\
175 \\
193 \\
262\end{array}$ & $13^{--}$ & $\begin{array}{c}.44 \\
\overline{-} \\
\overline{-} \\
3.3\end{array}$ & $\begin{array}{l}.- \\
.00 \\
.73 \\
-- \\
-.\end{array}$ & $\begin{array}{r}50 \\
-- \\
-10 \\
40\end{array}$ & $\begin{array}{r}190 \\
10 \\
--\end{array}$ & $\begin{array}{r}<20 \\
-- \\
=- \\
<20\end{array}$ & $\begin{array}{r}-- \\
90 \\
2 \\
--\end{array}$ \\
\hline $\begin{array}{l}.2 \\
.- \\
.1 \\
.2 \\
.2\end{array}$ & $\begin{array}{l}14 \\
14 \\
16 \\
19\end{array}$ & $\begin{array}{r}225 \\
-\overline{-} \\
240 \\
=-\end{array}$ & $\begin{array}{r}229 \\
-5 \\
241 \\
227 \\
196\end{array}$ & $\begin{array}{c}.00 \\
\cdots \\
.00 \\
-\end{array}$ & $\begin{array}{l}-- \\
-- \\
-- \\
--\end{array}$ & $\begin{array}{l}.0 \\
.00 \\
.00\end{array}$ & $\begin{array}{r}90 \\
-0 \\
130 \\
--\end{array}$ & $\begin{array}{l}-0 \\
30 \\
10\end{array}$ & $\begin{array}{l}=- \\
=- \\
=-\end{array}$ & $\begin{array}{r}-- \\
-\overline{-} \\
290 \\
--\end{array}$ \\
\hline $\begin{array}{l}.2 \\
.2 \\
.3\end{array}$ & $\begin{array}{l}23 \\
25 \\
20\end{array}$ & $\begin{array}{l}-\overline{1} \\
231\end{array}$ & $\begin{array}{l}381 \\
188 \\
242\end{array}$ & $\begin{array}{l}2.0^{--} \\
1.7\end{array}$ & ב- & $\begin{array}{c}1.3 \\
\ldots \\
\ldots\end{array}$ & $\begin{array}{r}-- \\
60 \\
120\end{array}$ & $\begin{array}{c}0 \\
--\end{array}$ & $\begin{array}{l}r- \\
<50 \\
<20\end{array}$ & $\begin{array}{l}<1 \\
-- \\
--\end{array}$ \\
\hline .3 & $20^{--}$ & 198 & 199 & $1.3^{--}$ & 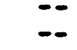 & 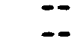 & 50 & $=$ & $<50$ & 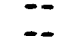 \\
\hline
\end{tabular}

$36 / 37 E-35 A 01$

\section{Ferry County}


TABLE 20.--Surmary of physical, biological, and major chemical-constituent data for ground-water samples from the Colville-Kettle subregion

[Values in milligrams per liter except as indicated umho, micromho; col $/ 100 \mathrm{~mL}$, colonies per 100 milliliter; $\mathrm{ug} / \mathrm{L}$, microgram per liter]

\begin{tabular}{|c|c|c|c|c|}
\hline \multirow{2}{*}{ Constituent } & \multirow{2}{*}{$\begin{array}{c}\text { Number of } \\
\text { sample sites }\end{array}$} & \multicolumn{3}{|c|}{ Trace element concentration } \\
\hline & & Maximum & Minimúm & Median \\
\hline Specific conductance (umho) & 14 & 635 & 208 & 354 \\
\hline $\mathrm{pH}$ (units) & 14 & 8.4 & 7.4 & 7.7 \\
\hline Temperature $\left({ }^{\circ} \mathrm{C}\right)$ & 14 & 13.6 & 8.3 & 10.6 \\
\hline Fecal-coliform bacteria (col $/ 100 \mathrm{~mL})$ & 6 & $<1$ & $<1$ & $<1$ \\
\hline Hardness (as $\mathrm{CaCO}_{3}$ ) & 14 & 280 & 96 & 173 \\
\hline Hardness, noncarbonate (as $\mathrm{CaCO}_{3}$ ) & 14 & 34 & 0 & 4.5 \\
\hline Calcium, dissolved & 14 & 86 & 23 & 47.5 \\
\hline Magnesium, magnesium & 14 & 21 & 7.8 & 13 \\
\hline Sodium, dissolved & 14 & 16 & 3.6 & 6.1 \\
\hline Sodium-adsorption ratio & 14 & .5 & .1 & .2 \\
\hline Potassium, dissolved & 14 & 4.5 & 1.0 & 2.2 \\
\hline Bicarbonate & 14 & 354 & 129 & 200 \\
\hline Carbonate & 14 & 0 & 0 & 0 \\
\hline Alkal inity (as $\mathrm{CaCO}_{3}$ ) & 14 & 290 & 106 & 164 \\
\hline Sulfate, dissolved & 14 & 54 & 6.3 & 19 \\
\hline Chloride, dissolved & 14 & 4.5 & .0 & 1.4 \\
\hline Fluoride, dissolved & 14 & .5 & .0 & .2 \\
\hline Silica, dissolved (as $\mathrm{SiO}_{2}$ ) & 14 & 31 & 14 & 19.5 \\
\hline Sol ids, dissolved (residue at $180^{\circ} \mathrm{C}$ ) & 8 & 284 & 150 & 214 \\
\hline Solids, dissolved (sum of constituents) & 14 & 381 & 144 & 213 \\
\hline Nitrate (as $N$ ) & 14 & 3.3 & .00 & .41 \\
\hline Iron, total recoverable (ug/L) & 8 & 130 & $<10$ & 55 \\
\hline Iron, dissolved (ug/L) & 7 & 190 & 0 & 10 \\
\hline Manganese, total recoverable $(\mathrm{ug} / \mathrm{L})$ & 5 & $<50$ & $<20$ & $<20$ \\
\hline Manganese, dissolved (ug/L) & 7 & 290 & $<1$ & 3 \\
\hline
\end{tabular}


TABLE 21.--Trace-element concentrations in ground-water samples from the Colville-Kettle subregion

\begin{tabular}{|c|c|c|c|c|c|c|c|}
\hline & & $\begin{array}{l}\text { ALUM- } \\
\text { INUM, }\end{array}$ & ALUM- & & & & CHRO- \\
\hline $\begin{array}{l}\text { LUCAL } \\
\text { IDENT- } \\
\text { I- } \\
\text { FIER }\end{array}$ & $\begin{array}{c}\text { UATE } \\
\text { UF } \\
\text { SAMPLE }\end{array}$ & $\begin{array}{l}\text { TOTAL } \\
\text { RECOV- } \\
\text { ERABLE } \\
\text { IUG/L }\end{array}$ & $\begin{array}{l}\text { INUM, } \\
\text { DIS- } \\
\text { SOLVED } \\
\text { (UG/L }\end{array}$ & $\begin{array}{c}\text { ARSENIC } \\
\text { UIS- } \\
\text { SOLVED } \\
\text { (UG/L }\end{array}$ & $\begin{array}{l}\text { BARIUM, } \\
\text { DIS- } \\
\text { SOLVEO } \\
\text { (UG/L }\end{array}$ & $\begin{array}{l}\text { CADMIUM } \\
\text { DIS- } \\
\text { SOLVED } \\
\text { IUG/L }\end{array}$ & $\begin{array}{l}\text { MIUM, } \\
\text { OIS- } \\
\text { SOLVED } \\
\text { (UG/L }\end{array}$ \\
\hline & & AS AL) & AS AL) & AS AS) & AS BA) & AS CD) & AS $(R)$ \\
\hline
\end{tabular}

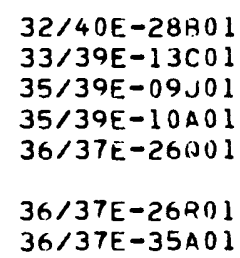
$71-10-00$
$79-06-21$
$71-10-00$
$73-06-22$
$67-\dot{y} \dot{y}-20$
71) $-11-11$
$67-0 y-20$

$<10$
120
--

$--$

$-\frac{7}{7}$
-2
$<10$

100
--
100
$<1000$

$\begin{array}{lr}-- & <30 \\ <1 & 0 \\ -- & <30 \\ <1 & 0 \\ <5 & <50\end{array}$

30

$--$

$<10$

$<1000$

$<5 \quad<30$

$\begin{array}{llcccc} & & \\ \text { COPPER, } & \text { LEAD, } & \text { MERCURY } & \text { SELE- } & \\ \text { NIUM, } & \text { SILVER, } & \text { ZINC, } \\ \text { DIS- } & \text { DIS- } & \text { DIS- } & \text { DIS- } & \text { DIS- } & \text { DIS- } \\ \text { SOLVED } & \text { SOLVED } & \text { SOLVEO } & \text { SOLVED } & \text { SULVED } & \text { SOLVEU } \\ (U G / L & (U G / L & (U G / L & (U G / L & (U G / L & (U G / L \\ \text { AS CU) } & \text { ASPB) } & \text { AS HG) } & \text { AS SE) } & \text { AS AG) } & \text { AS ZN) }\end{array}$

$\begin{array}{rrrrrr}<50 & <100 & -- & -- & -- & <10 \\ 0 & 00 & -4 & 0 & 0 & <3 \\ <50 & <100 & -- & -- & -- & <10 \\ 1 & 00 & -2 & 0 & 0 & <3 \\ <400 & <40 & -- & <10 & <40 & <500 \\ <50 & <100 & -- & -- & -- & 70 \\ <400 & <40 & -- & <10 & <40 & <500\end{array}$


TABLE 22.--Physical, biological, and major chemical-constituent data for ground-water samples from the Pend Oreille subregion

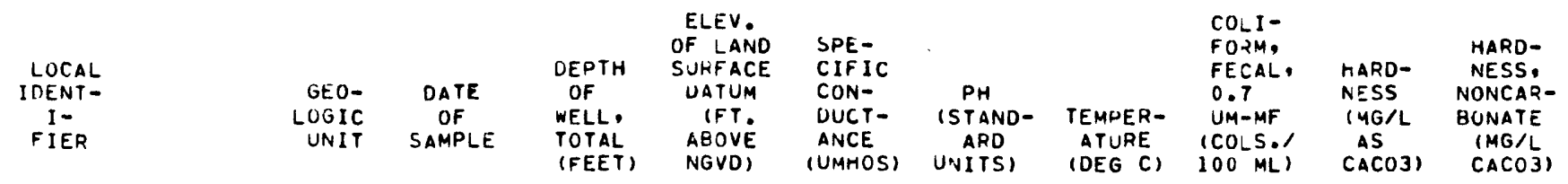

\begin{tabular}{|c|c|c|}
\hline $\begin{array}{l}31 / 45 E-24301 \\
31 / 45 E-34601 \\
32 / 44 E-07801 \\
32 / 44 E-27601 \\
32 / 44 E-36 C 01\end{array}$ & $\begin{array}{l}11 \text { UALVM } \\
112 G L C V \\
11 \text { UALVM } \\
11 \text { OOLVM } \\
11 \text { OALVM }\end{array}$ & $\begin{array}{l}79-07-18 \\
7 y-08-03 \\
79-07-18 \\
7 y-08-02 \\
79-08-02\end{array}$ \\
\hline $\begin{array}{l}32 / 45 E-32 E 01 \\
32 / 45 E-32401 \\
32 / 45 E-33401 \\
32 / 45 E-34001\end{array}$ & $\begin{array}{l}11 \text { UALVM } \\
110 A L V M \\
110 A L V M \\
110 A L V M \\
11 \text { UALVM }\end{array}$ & $\begin{array}{l}61-05-02 \\
70-11-11 \\
71-05-26 \\
79-08-02 \\
7 y-08-03\end{array}$ \\
\hline $\begin{array}{l}33 / 43 E-11 N O 1 \\
35 / 43 E-12 L O 1 \\
36 / 43 E-35 F 01 \\
37 / 43 E-29 \times 01\end{array}$ & $\begin{array}{l}110 A L V M \\
11 U A L V M \\
110 A L V M \\
11 \text { OALVM }\end{array}$ & $\begin{array}{l}79-08-03 \\
79-08-03 \\
7 y-08-02 \\
7 y-08-02\end{array}$ \\
\hline
\end{tabular}

$\begin{array}{rrr}<1 & 144 & 14 \\ <1 & 109 & 9 \\ <1 & 242 & 2 \\ <1 & 178 & 0 \\ <1 & 143 & 3 \\ -- & 50 & 1 \\ -- & 50 & 1 \\ -- & 46 & 0 \\ <1 & 96 & 9 \\ <1 & 119 & 0 \\ & & \\ <1 & 179 & 0 \\ <1 & 289 & 9 \\ <1 & 263 & 3 \\ <1 & 220 & 0\end{array}$

$$
\begin{aligned}
& \text { LUCAL } \\
& \text { IDENT- } \\
& \text { I- } \\
& \text { FIER }
\end{aligned}
$$

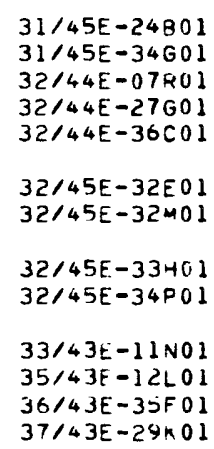

$\begin{array}{rrrrr}175 & 2166.00 & 330 & 7.9 & 10.5 \\ 208 & -- & 211 & 8.2 & 16.2 \\ 77 & -- & 470 & 7.5 & 10.9 \\ 99 & -- & 360 & 8.0 & 12.4 \\ 57 & -- & 292 & 7.8 & 12.2 \\ 37 & 2060.00 & 120 & 6.7 & 9.5 \\ 50 & 2065.00 & 123 & 6.8 & 7.2 \\ 50 & 2065.00 & 116 & 8.1 & 8.7 \\ 91 & -- & 200 & 8.2 & 11.4 \\ 171 & -- & 240 & 8.0 & 11.4 \\ 160 & -- & 357 & 7.8 & 12.0 \\ 60 & -- & 545 & 7.2 & 15.0 \\ 87 & 2080.00 & 504 & 7.4 & 9.8 \\ 90 & -- & 450 & 6.7 & 9.1\end{array}$

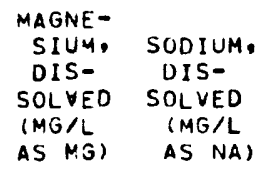

CAR-
BONATE
FET $-F L D$
(MC/L
AS CU3)

AL $<A=$ LINITY FIELD (MG/L AS CACO3)
SULFATE OISSOLVED ( $M G / L$ AS $\mathrm{SO}_{4}$ )
CHLORIDE. OISSOLVED (MG/L AS CL)
AS K) $\mathrm{ACO}$ )

$\begin{array}{llll}0.1 & .3 & 2.2 & 159 \\ 3.6 & .2 & 1.5 & 122 \\ 5.5 & .2 & 2.7 & 293 \\ 11 & .4 & 2.4 & 232 \\ 4.9 & .2 & 1.5 & 171 \\ 4.2 & .3 & .9 & 60 \\ 4.6 & .3 & .9 & 60 \\ . .0 & . . & .- & 61 \\ 3.1 & .1 & 1.0 & 106 \\ 3.3 & .1 & 1.5 & 146 \\ 6.6 & .2 & 2.7 & 220 \\ 4.1 & .1 & 2.6 & 341 \\ 5.2 & .1 & 1.6 & 317 \\ 9.4 & .3 & 2.1 & 258\end{array}$

$\begin{array}{rr}-- & 130 \\ -- & 100 \\ 0 & 240 \\ 0 & 190 \\ 0 & 140 \\ & \\ 0 & 49 \\ 0 & 49 \\ 0 & 50 \\ 0 & 97 \\ 0 & 120 \\ & \\ 0 & 180 \\ 0 & 240 \\ 0 & 260 \\ 0 & 220\end{array}$

$\begin{array}{lr}11 & 7.6 \\ 14 & .5 \\ 7.9 & 1.9 \\ 15 & .8 \\ 14 & .6 \\ 8.6 & .8 \\ 7.6 & 1.7 \\ -. & .9 \\ 15 & .5 \\ 13 & 1.2 \\ & \\ 14 & .5 \\ 22 & 1.2 \\ 16 & 1.0 \\ 22 & .7\end{array}$


TABLE 22.--Continued
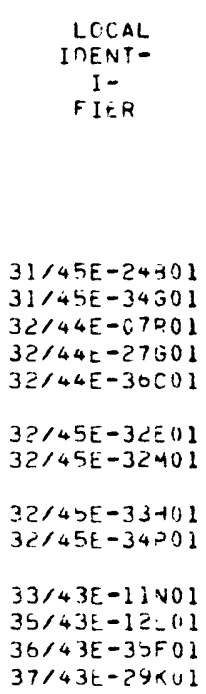

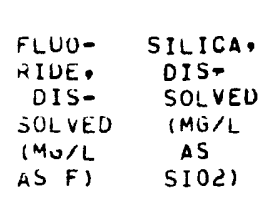

\begin{tabular}{|c|c|c|}
\hline SULIUS, & SOLIOS, & \\
\hline ESIUUE & SUM of & NITRO- \\
\hline AT 180 & CONSTI- & GEN, \\
\hline CIS- & 政 & TOTAL \\
\hline $\begin{array}{l}\text { SOLVED } \\
(M G / L)\end{array}$ & $\begin{array}{l}\text { SOLVED } \\
\text { (MG/L) }\end{array}$ & $\begin{array}{c}(M U / L \\
\text { AS NO3) }\end{array}$ \\
\hline
\end{tabular}

NITROGEN, $\mathrm{NO} 2+\mathrm{NO}_{3}$ DISSOLVEO ( $M G / L$ AS NI
IRON, TOTAL RECOV-

ERABLE IUG/L AS FE)

IRON.
OIS-
SOLVED
(UEAL
AS FE)

MANGANESE, TOTAL RECOVERABLE (UGLL AS MN)
MANGANESE, DISSOLVED (UG/L $\triangle S M V)$

\begin{tabular}{|c|c|c|c|c|c|c|c|c|}
\hline 24 & - & 162 & -- & 5.6 & -- & $<20$ & - & 7 \\
\hline 15 & - & 133 & - & .18 & -- & 80 & - & 2 \\
\hline 18 & -- & 269 & - & .05 & -- & 130 & $=-$ & 320 \\
\hline 19 & - & 227 & - & .17 & - & 140 & -- & 240 \\
\hline 17 & $\cdots$ & 174 & $\cdots$ & .06 & $\cdots$ & $<10$ & $\cdots$ & 590 \\
\hline 24 & 89 & 86 & 1.8 & -- & $<10$ & -- & $\cdots$ & $\cdots$ \\
\hline 23 & 85 & 85 & 2.7 & -- & 60 & -- & $<20$ & $\cdots$ \\
\hline-- & $\cdots$ & -- & -- & -- & -- & -- & $\cdots$ & -- \\
\hline 14 & $\cdots$ & 119 & -- & .08 & $\cdots$ & 110 & -- & 1 \\
\hline 13 & -- & 147 & -- & .14 & -- & 340 & -- & 7 \\
\hline 18 & -- & 213 & $\cdots$ & .02 & -- & 360 & -- & 100 \\
\hline 17 & -- & 316 & -- & .04 & $\cdots$ & 230 & -- & $<10$ \\
\hline 26 & -- & 299 & $\cdots$ & 1.2 & $\cdots$ & $<10$ & $\cdots$ & $<1$ \\
\hline 21 & $\cdots$ & 259 & - & .02 & $\cdots$ & 1800 & - & 180 \\
\hline
\end{tabular}


TABLE 23.--Summary of physical, biological, and major chemical-constituent data for ground-water samples from the Pend Oreille subregion

[Values in milligrams per liter except as indicated umho, micromho; col/100 mL, colonies per 100 milliliter; ug/L, microgram per liter]

\begin{tabular}{|c|c|c|c|c|}
\hline Constituent & $\begin{array}{l}\text { Number of } \\
\text { sample sites }\end{array}$ & $\frac{\text { Trace ele }}{\text { Maximum }}$ & $\frac{\text { ent conce }}{\text { Minimum }}$ & $\frac{\text { tration }}{\text { Median }}$ \\
\hline Specific conductance (umho) & 13 & 545 & 116 & 330 \\
\hline $\mathrm{pH}$ (units) & 13 & 8.2 & 6.7 & 7.8 \\
\hline Temperature $\left({ }^{\circ} \mathrm{C}\right)$ & 13 & 16.2 & 7.2 & 11.4 \\
\hline Fecal-coliform bacteria (col/100 mL) & 11 & $<1$ & $<1$ & $<1$ \\
\hline Hardness (as $\mathrm{CaCO}_{3}$ ) & 13 & 290 & 46 & 140 \\
\hline Hardness, noncarbonate (as $\mathrm{CaCO}_{3}$ ) & 13 & 10 & 0 & 0 \\
\hline Calcium, dissolved & 13 & 78 & 12 & 42 \\
\hline Magnesium, dissolved & 13 & 23 & 3.6 & 10 \\
\hline Sodium, dissolved & 13 & 11 & 3.1 & 4.9 \\
\hline Sodium-adsorption ratio & 13 & .4 & .1 & .2 \\
\hline Potassium, dissolved & 13 & 2.7 & .9 & 1.6 \\
\hline Bicarbonate & 13 & 341 & 60 & 171 \\
\hline Carbonate & 13 & 0 & 0 & 0 \\
\hline Alkal inity $\left(\right.$ as $\mathrm{CaCO}_{3}$ ) & 13 & 280 & 49 & 140 \\
\hline Sulfate, dissolved & 13 & 22 & 7.6 & 14 \\
\hline Chloride, dissolved & 13 & 7.6 & .5 & .8 \\
\hline Fluoride, dissolved & 13 & .3 & .1 & .1 \\
\hline Silica, dissolved (as $\mathrm{SiO}_{2}$ ) & 13 & 26 & 13 & 18 \\
\hline Solids, dissolved (residue at $180^{\circ} \mathrm{C}$ ) & 2 & 89 & 85 & -- \\
\hline Solids, dissolved (sum of constituents) & 13 & 316 & 88 & 207 \\
\hline Nitrate (as N) & 13 & 5.6 & .02 & .14 \\
\hline Iron, total recoverable $(\mathrm{ug} / \mathrm{L})$ & 2 & 60 & $<10$ & -- \\
\hline Iron, dissolved (ug/L) & 11 & 1,800 & 0 & 130 \\
\hline Manganese, total recoverable (ug/L) & 1 & $<20$ & $<20$ & -- \\
\hline Manganese, dissolved (ug/L) & 10 & 590 & $<1$ & 10 \\
\hline
\end{tabular}


TABLE 24.--Trace-element concentrations in ground-water samples from the Pend Oreille subregion

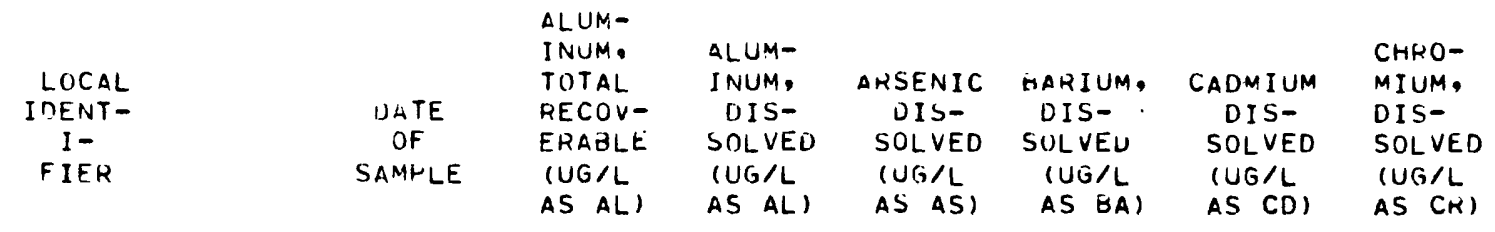

$\frac{0}{0}$

$-\frac{3}{1}$

$\frac{60}{50}$

$-2$

0
$<10$
10

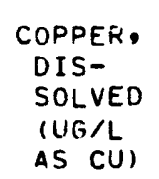

LEAD,
DIS-
SOLVED
(UG/L
AS PB)

MERCURY
DIS-
SOLVED
(UG/L
AS HG)

SELE-

NIUM,

DIS-

SOLVED

(UG/L

SILVER,

UIS-

SULVED

(UG/L

AS $A G)$

ZINC,

DIS-

SOLVED

(UG/L

(UG/L

AS SE)

AS $2 N$ )

$\begin{array}{rrrrrr}15 & 2 & .0 & 0 & 0 & 70 \\ <50 & <100 & -0 & -- & -- & 220 \\ 2 & 2 & .0 & 2 & 0 & 150\end{array}$




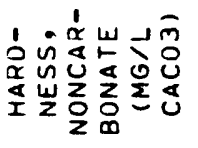

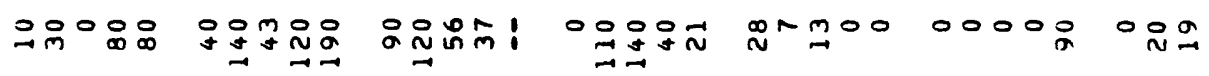

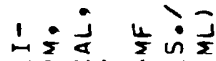
家品出:

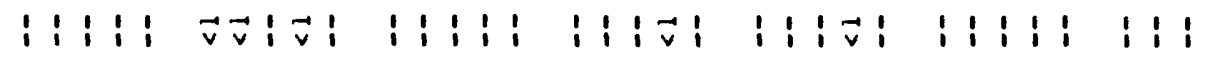

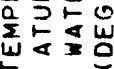

\section{过亲离离}

\section{$\infty \simeq$}

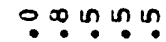

\section{to on in}

in 0 in 00

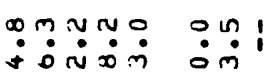

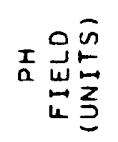

$\sin \sin i \frac{\sin x}{4}$

$\underset{\sim}{\sim} \dot{\sim} \underset{\sim}{0}$

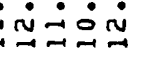

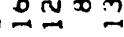

-

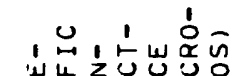

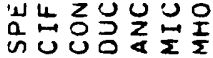

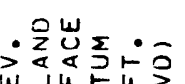

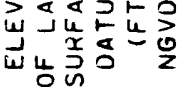

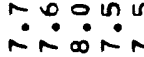

trisit

$\because \infty 0^{\infty}: 0$

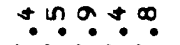

onmon

๓กำ? ํ.?

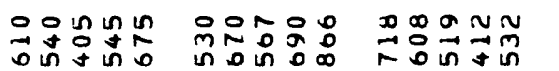

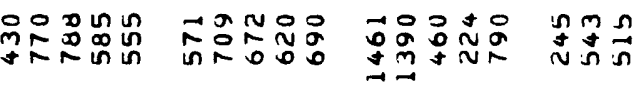

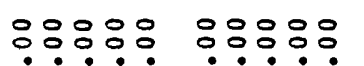

$\dot{0} \dot{0} \dot{0} \dot{0}$

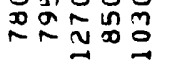

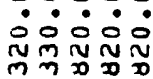

$\therefore \circ: \circ:$

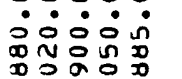

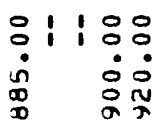

유유 웅

ग0

o엉

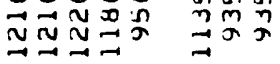

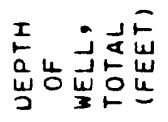

$\overrightarrow{0} \underset{N}{n}+\infty) \infty$

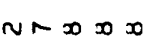

드늠윰요

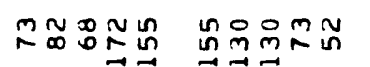

$\underset{m}{\sim} \underset{n}{\sim} \stackrel{\infty}{\sim} \stackrel{\sim}{\sim} \underset{m}{\leftrightarrows} m$

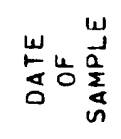

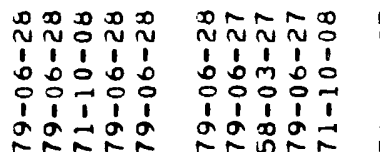

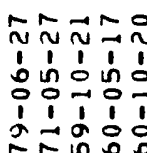

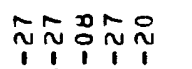

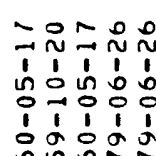

กํํํำ ํㅜㄴ

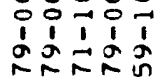

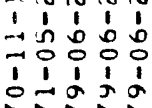

bon

ín

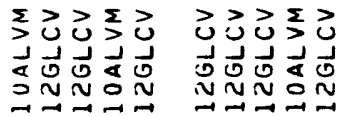

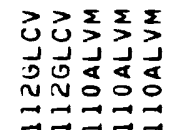

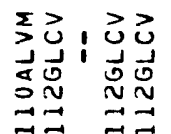

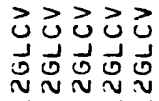

己己己文

岁苑艺

$\exists \Xi \Xi \Xi \Xi$

$コ コ コ コ コ$

ヘコーコー
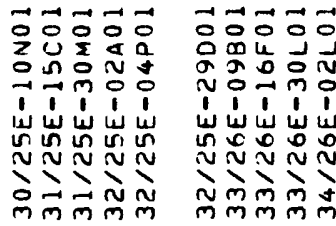

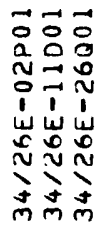
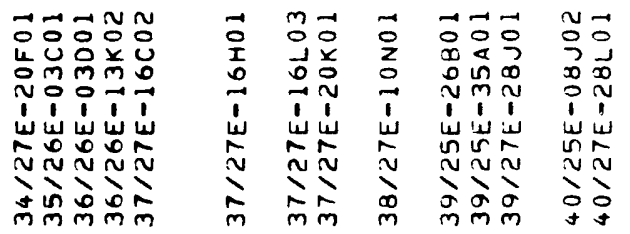


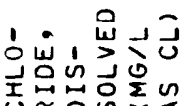

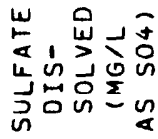

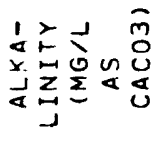

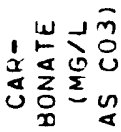

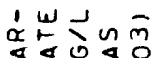

过艺造证

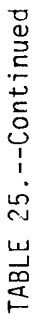

新的岸大的

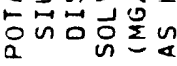

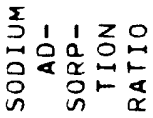

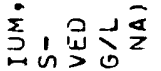

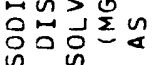

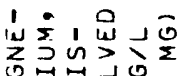

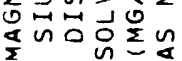

志, 崖这

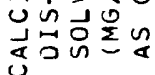

古它。点

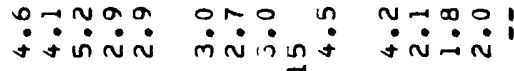

$\dot{m} \dot{\min } \dot{\sim} \dot{\infty}$

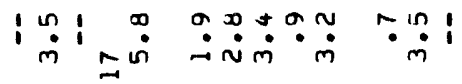

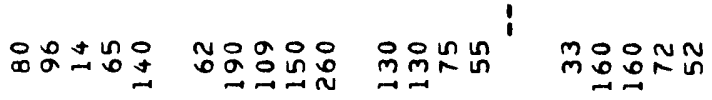

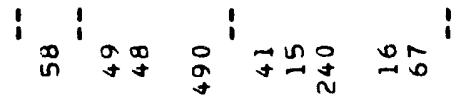

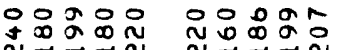

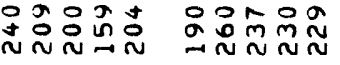

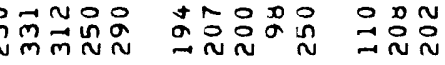

000000000

00000

00000

0000000000000

우웅

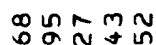

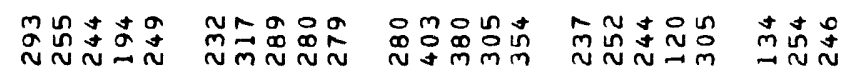

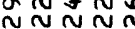

N $\sim$ N

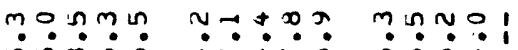

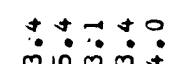

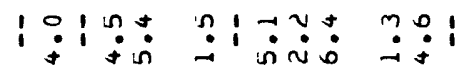

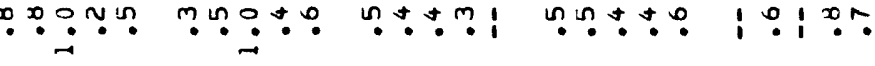

N

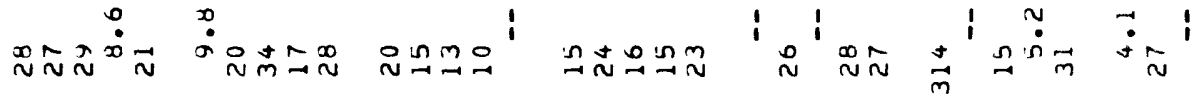

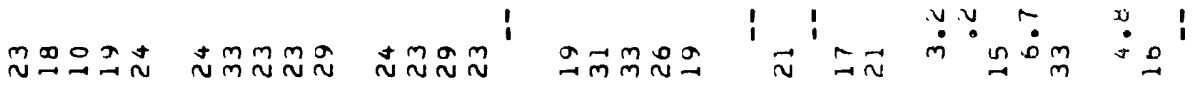

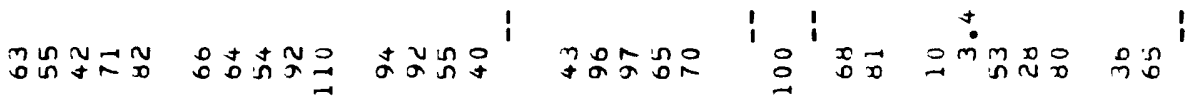
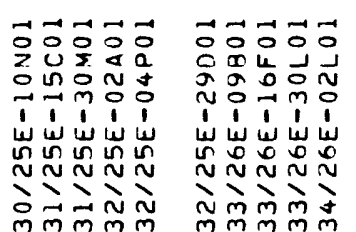

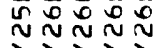

iिm户िलें

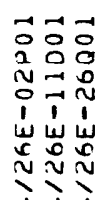

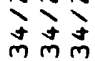
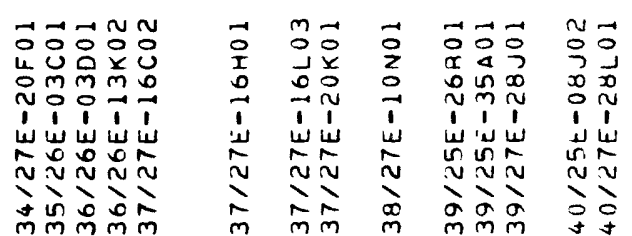
这的跳

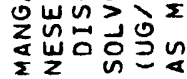

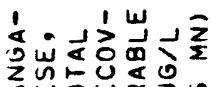

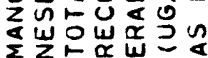

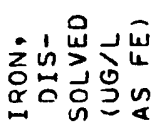

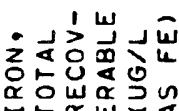

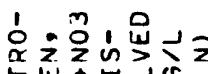

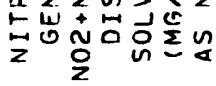

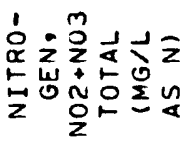

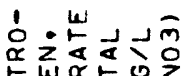

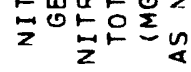

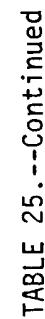

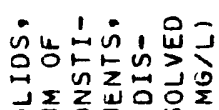

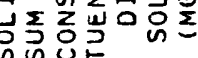

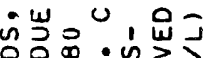

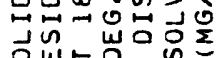

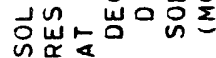

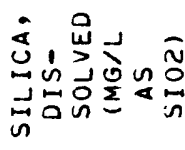

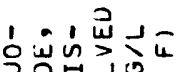

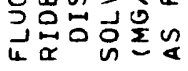

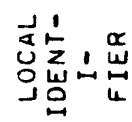

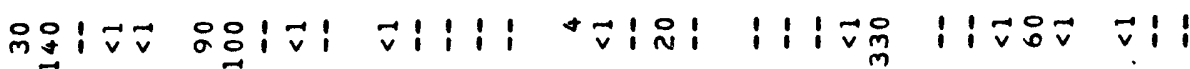

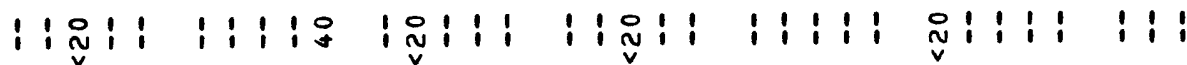

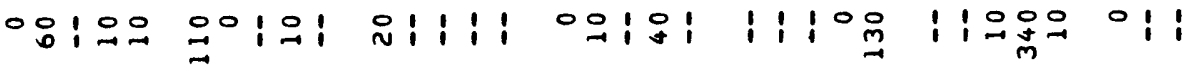

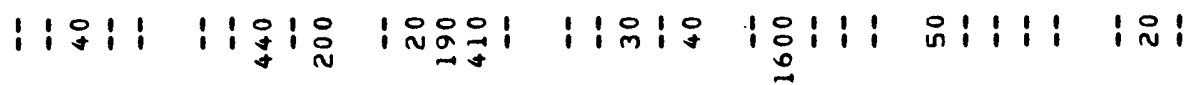

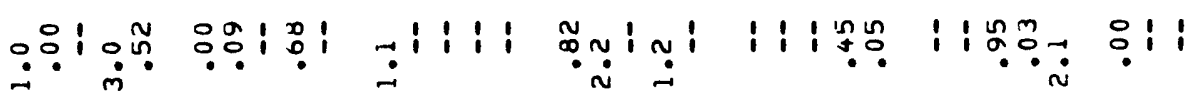

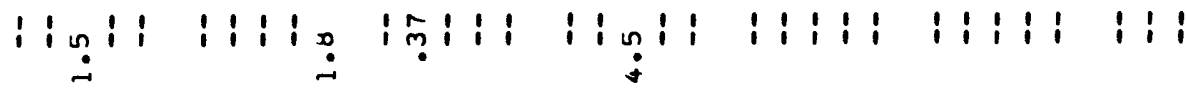

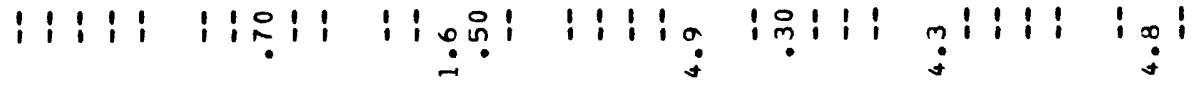

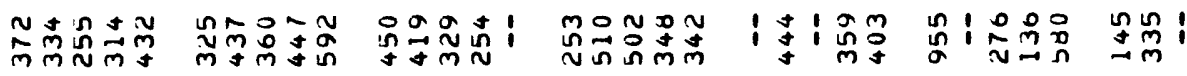

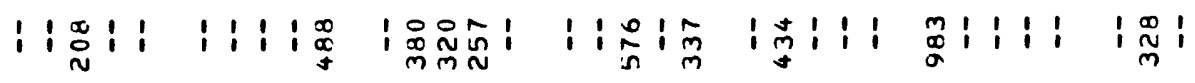

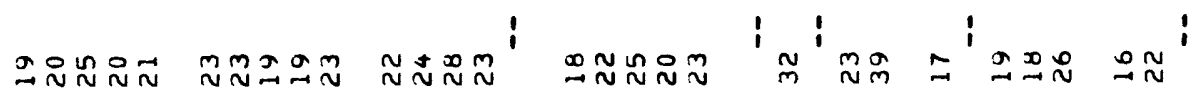

若

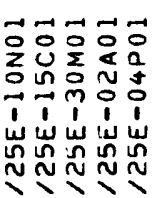

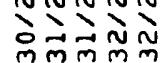

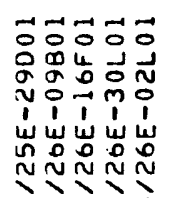

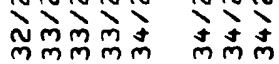

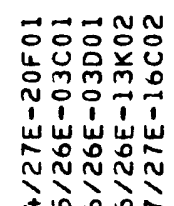

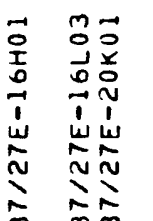

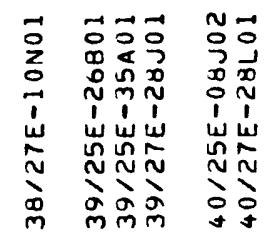


TABLE 26.--Summary of physical, biological, and major chemical-constituent data for ground-water samples from the Okanogan Region

[Values in milligrams per liter except as indicated umho, micromho; col $/ 100 \mathrm{~mL}$, colonies per 100 milliliter; ug/L, microgram per liter]

\begin{tabular}{|c|c|c|c|c|}
\hline Constituent & $\begin{array}{c}\text { Number of } \\
\text { sample sites }\end{array}$ & $\frac{\text { Trace elen }}{\text { Maximum }}$ & $\begin{array}{l}\text { ent concer } \\
\text { Minimum }\end{array}$ & $\begin{array}{l}\text { tration } \\
\text { Median }\end{array}$ \\
\hline Specific conductance (umho) & 33 & 1,461 & 224 & 585 \\
\hline $\mathrm{pH}$ (units) & 33 & 8.2 & 7.2 & 7.6 \\
\hline Temperature $\left({ }^{\circ} \mathrm{C}\right)$ & 32 & 16.3 & 8.2 & 12.4 \\
\hline Fecal-coliform bacteria (col/100 mL) & 5 & $<1$ & $<1$ & $<1$ \\
\hline Hardness (as $\mathrm{CaCO}_{3}$ ) & 33 & 390 & 9 & 257 \\
\hline Hardness, noncarbonate (as $\left.\mathrm{CaCO}_{3}\right)$ & 32 & 190 & 0 & 29 \\
\hline Calcium, dissolved & 29 & 110 & 3.4 & 65 \\
\hline Magnesium, dissolved & 29 & 33 & .2 & 23 \\
\hline Sodium, dissolved & 28 & 314 & 4.1 & 20.5 \\
\hline Sodium-adsorption ratio & 28 & 22 & .2 & .5 \\
\hline Potassium, dissolved & 28 & 7.8 & 1.3 & 4.8 \\
\hline Bicarbonate & 33 & 403 & 120 & 252 \\
\hline Carbonate & 33 & 0 & 0 & 0 \\
\hline Alkalinity (as $\mathrm{CaCO}_{3}$ ) & 33 & 331 & 98 & 207 \\
\hline Sulfate, dissolved & 28 & 490 & 14 & 73.5 \\
\hline Chloride, dissolved & 29 & 17 & .7 & 3.2 \\
\hline Fluoride, dissolved & 28 & .6 & .1 & .3 \\
\hline Silica, dissolved (as $\mathrm{SiO}_{2}$ ) & 28 & 39 & 16 & 22 \\
\hline Solids, dissolved (residue at $180^{\circ} \mathrm{C}$ ) & 10 & 983 & 208 & 359 \\
\hline Solids, dissolved (sum of constituents) & 28 & 955 & 136 & 340 \\
\hline Nitrate (as N) & 28 & 4.5 & .00 & .60 \\
\hline Iron, total recoverable (ug/L) & 11 & 1,600 & 20 & 50 \\
\hline Iron, dissolved $(\mathrm{ug} / \mathrm{L})$ & 17 & 340 & 0 & 10 \\
\hline Manganese, total recoverable (ug/L) & 5 & 40 & $<20$ & $\cdot<20$ \\
\hline Manganese, dissolved (ug/L) & 17 & 330 & $<1$ & $<1$ \\
\hline
\end{tabular}


TABLE 27.--Trace-element concentrations in ground-water samples from the Okanogan Region

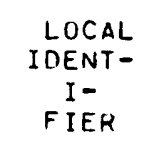

$30 / 25 E-10 N O 1$

$31 / 25 E-30 M 01$

$33 / 26 E-09 B 01$

$34 / 26 E-02 L 01$

$34 / 26 E-11001$

$36 / 26 E-03001$

$37 / 27 E-16 L 03$

$38 / 27 E-1$ ONOI

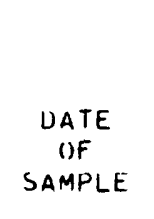

$79-06-28$
$71-10-08$
$79-06-27$
$71-10-08$
$71-05-27$
$71-10-08$
$74-06-26$
$70-11-12$
ALUM-

INUM, ALUM-

TOTAL INUM,

RECOV - DIS-

ERABLE SOLVED

(UG/L

AS AL,
IUG/L

AS AL)
ARSENIC

DIS-

SOLVED

(UG/L

AS AS)
BARIUM, DIS-

SOLVEO

(UG/L

AS $B A$ )
CADMIUM

DIS-

SOLVED

IUG/L

AS CD I
CHRO-

MIUM, COPDER, DIS- DIS-

SOLVED SOLVED IUG/L IUG/L AS $(R)$ AS CUI

Okanogan County

$\begin{array}{rrrrrrr}-- & 10 & 5 & 50 & <1 & 0 & 3 \\ 30 & -- & -- & -- & -- & <30 & <50 \\ -- & 20 & 6 & 40 & <- & 0 & 2 \\ 50 & -- & -- & -- & -- & <30 & <50 \\ <10 & -- & -- & -- & -- & <30 & <50 \\ 90 & -- & -- & -- & -- & <30 & <50 \\ -- & 10 & 2 & 40 & <1 & 0 & 1 \\ 30 & -- & -- & -- & -- & <30 & <50\end{array}$

$\begin{array}{lcccc} & \text { SELE- } & & \\ \text { LEAD, } & \text { MERCURY } & \text { NIUM, } & \text { SILVER, } & \text { ZINC, } \\ \text { DIS- } & \text { DIS- } & \text { DIS- } & \text { DIS- } & \text { DIS- } \\ \text { SOLVED } & \text { SOLVED } & \text { SOLVED } & \text { SOLVED } & \text { SOLVED } \\ (U G / L & (U G / L & \text { (UG/L } & (U G / L & (U G / L \\ \text { ASPB) } & \text { AS HG) } & \text { AS SE) } & \text { AS AG) } & \text { AS ZN) }\end{array}$

$\begin{array}{rrrrr}0 & .0 & 12 & 0 & 3 \\ <100 & -- & -- & -- & <10 \\ 0 & -0 & -1 & -- & <10 \\ <100 & -- & -- & -- & <10 \\ <100 & - & -- & -- & <10 \\ <100 & -- & -0 & 0 & 5 \\ 0 & -0 & -- & -- & 70\end{array}$


TABLE 28.--Physical, biological, and major chenical-constituent data for ground-water samples from the Methow Region

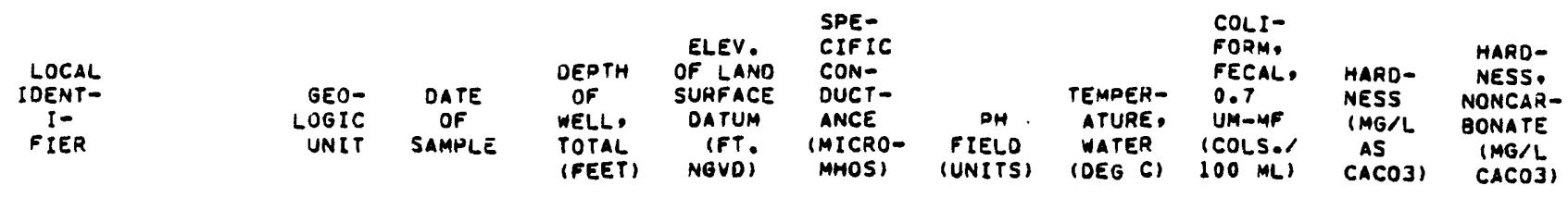

$30 / 22 E-13601$

$30 / 23 E-06 K 01$

$31 / 22 E-26 N O 1$

$32 / 22 E-1$ OFO

$33 / 22 E-08 N O 1$

33/22E-1700

$35 / 21 E-32 K O$

$36 / 19 E-26801$

$\begin{array}{lrr}110 A L V M & 79-07-10 & 52 \\ 110 A L V M & 70-11-12 & 75 \\ 110 A L V M & 71-05-27 & 75 \\ 110 A L V M & 79-07-10 & 41 \\ 110 A L V M & 79-07-10 & 105 \\ & & \\ 110 A L V M & 59-10-20 & 50 \\ 110 A L V M & 60-05-18 & 50 \\ 110 A L V M & 79-07-09 & 100 \\ 110 A L V M & 79-07-10 & 103 \\ 1 \text { JOALVM } & 59-09-16 & 50\end{array}$

IIUALVM
Okanogan County

$\begin{array}{rrrrrr}1040.00 & 215 & 7.8 & 11.0 & <1 & 89 \\ -0 & 230 & 7.7 & 9.6 & - & 107 \\ -0 & 113 & 7.9 & 10.8 & - & 46 \\ 1210.00 & 240 & 7.6 & 11.0 & <1 & 100 \\ 1490.00 & 290 & 7.8 & 12.3 & - & 82 \\ -- & 215 & 7.2 & 11.0 & - & 100 \\ -0 & 190 & 7.2 & 8.0 & -\infty & 98 \\ 1608.71 & 176 & 7.4 & 8.8 & <1 & 74 \\ 1830.00 & 160 & 7.3 & 10.5 & <1 & 71 \\ -- & 111 & 7.1 & -\infty & -\infty & 49\end{array}$

\begin{tabular}{|c|c|c|}
\hline & MAGNE- & \\
\hline $\begin{array}{l}\text { CALCIUM } \\
\text { DIS- } \\
\text { SOLVED } \\
\text { IMG/L }\end{array}$ & $\begin{array}{l}\text { SIUM, } \\
\text { DIS- } \\
\text { SOLVED } \\
\text { IMG/L }\end{array}$ & $\begin{array}{l}\text { SOOIUM, } \\
\text { DIS- } \\
\text { SOLVED } \\
\text { (MG/L }\end{array}$ \\
\hline AS (A) & AS MGI & AS NAI \\
\hline
\end{tabular}

$\begin{array}{ccc}\text { SODIUM } & \text { POTAS- } \\ \text { AO- } & \text { SIUM, BICAR- } \\ \text { SORP- } & \text { DIS- } & \text { BONATE } \\ \text { TION } & \text { SOLVED } \\ \text { (MG/L } \\ \text { RATIO } & \text { (MG/L } & \text { AS } \\ & \text { AS K) } & \text { HCOJ) }\end{array}$

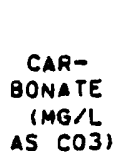

ALKA- SULFATE
LINITY DIS-
(MG/L SOLVED
AS
CACO3) AS SOL)

CHLORIDE, DISSOLVED (MG/L AS CL)

\begin{tabular}{|c|c|c|c|c|c|c|c|c|c|}
\hline $\begin{array}{l}28 \\
33 \\
14 \\
31 \\
21\end{array}$ & $\begin{array}{l}4.7 \\
5.8 \\
2.8 \\
6.2 \\
7.1\end{array}$ & $\begin{array}{r}5.4 \\
6.0 \\
-0 \\
6.0 \\
4.6\end{array}$ & $\begin{array}{l}.2 \\
.3 \\
-. \\
.3 \\
.2\end{array}$ & $\begin{array}{l}1: 3 \\
1.0 \\
0 . \\
1: 2 \\
1.4\end{array}$ & $\begin{array}{r}109 \\
128 \\
70 \\
134 \\
99\end{array}$ & $\begin{array}{l}0 \\
0 \\
0 \\
0 \\
0\end{array}$ & $\begin{array}{r}89 \\
105 \\
57 \\
110 \\
81\end{array}$ & $\begin{array}{l}10 \\
13 \\
11 \\
17\end{array}-$ & $\begin{array}{r}1.0 \\
.6 \\
1.0 \\
.8 \\
1.1\end{array}$ \\
\hline $\begin{array}{l}33 \\
23 \\
21 \\
18\end{array}$ & $\begin{array}{r}4.1 \\
.0 \\
4.1 \\
4.4 \\
1.0\end{array}$ & $\begin{array}{r}4.3 \\
.0 \\
3.0 \\
2.7 \\
1.9\end{array}$ & $\begin{array}{l}.2 \\
-.2 \\
.2 \\
.1 \\
.1\end{array}$ & $\begin{array}{r}1.2 \\
.5 \\
.5 \\
.5 \\
.5\end{array}$ & $\begin{array}{r}111 \\
109 \\
87 \\
91 \\
62\end{array}$ & $\begin{array}{l}0 \\
0 \\
0 \\
0 \\
0\end{array}$ & $\begin{array}{l}91 \\
89 \\
71 \\
75 \\
51\end{array}$ & $\begin{array}{r}14 \\
9.4 \\
6.0 \\
3.7\end{array}$ & $\begin{array}{r}.8 \\
-. \\
1.3 \\
.5 \\
.0\end{array}$ \\
\hline
\end{tabular}

\begin{tabular}{|c|c|c|c|c|c|c|c|c|c|}
\hline $\begin{array}{l}\text { FLUO- } \\
\text { RIDE, } \\
\text { DIS- } \\
\text { SOLVEU } \\
\text { IMG/L }\end{array}$ & $\begin{array}{l}\text { SILICA, } \\
\text { OIS- } \\
\text { SOLVEO } \\
\text { IMG/L } \\
\text { AS }\end{array}$ & $\begin{array}{l}\text { SOLIOS, } \\
\text { RESIOUE } \\
\text { AT } 180 \\
\text { UEG. C } \\
\text { DIS- } \\
\text { SOLVED }\end{array}$ & $\begin{array}{l}\text { SOLIDS, } \\
\text { SUM OF } \\
\text { CONSTI- } \\
\text { TUENTS, } \\
\text { OIS- } \\
\text { SOLVED }\end{array}$ & $\begin{array}{l}\text { NITRO- } \\
\text { GENE } \\
\text { NITRATE } \\
\text { TOTAL } \\
\text { IMG/L }\end{array}$ & $\begin{array}{c}\text { NITRO- } \\
\text { GEN. } \\
\text { NOZ } \rightarrow \text { NO } 3 \\
\text { OIS- } \\
\text { SOLVED } \\
\text { IMG/L }\end{array}$ & $\begin{array}{l}\text { IRON } \\
\text { TOTAL } \\
\text { RECOV- } \\
\text { ERABLE } \\
\text { IUG/L }\end{array}$ & $\begin{array}{l}\text { IRON, } \\
\text { DIS- } \\
\text { SOLVED } \\
\text { IUG/L }\end{array}$ & $\begin{array}{l}\text { MANGA- } \\
\text { NESE, } \\
\text { TOTAL } \\
\text { RECOV- } \\
\text { ERABLE } \\
\text { IUG/L }\end{array}$ & $\begin{array}{l}\text { MANGA- } \\
\text { NESE, } \\
\text { OIS- } \\
\text { SOLVED } \\
\text { IUG/L }\end{array}$ \\
\hline$\Rightarrow$ & S(02) & IMG & (MG/L) & AS $\mathrm{NO}^{31}$ & AS NI & AS FE) & AS & AS MNI & AS MNI \\
\hline
\end{tabular}

\begin{tabular}{|c|c|c|c|c|c|c|c|c|}
\hline 13 & $\ddot{0}$ & 121 & $=$ & .77 & $=$ & 10 & $\ddot{B}$ & $\leq 1$ \\
\hline $13 \ldots$ & 138 & $13 B$ & 2.5 & -- & $\begin{array}{l}20 \\
--\end{array}$ & $=$ & $<20$ & $=$ \\
\hline 15 & -- & 142 & $\cdots$ & .85 & -- & 0 & -- & $<$ \\
\hline 14 & - & 118 & - & .55 & $\cdots$ & $<0$ & - & $<1$ \\
\hline 13 & 127 & 128 & 2.1 & -- & 20 & -- & -- & - \\
\hline $11^{--}$ & $=$ & $\overline{9 \theta}$ & -- & .53 & $=$ & 0 & - & \\
\hline 9.9 & - & 90 & $\cdots$ & .09 & -- & 0 & 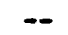 & \\
\hline 9.0 & 65 & 66 & .50 & -- & 20 & -- & -- & \\
\hline
\end{tabular}


TABLE 29.--Summary of physical, biological, and major chemical-constituent data for ground-water samples from the Methow Region

[Values in milligrams per liter except as indicated umho, micromho; col/100 mL, colonies per 100 milliliter; ug/L, microgram per liter]

\begin{tabular}{|c|c|c|c|c|}
\hline \multirow[b]{2}{*}{ Constituent } & \multirow{2}{*}{$\begin{array}{l}\text { Number of } \\
\text { sample sites }\end{array}$} & \multicolumn{3}{|c|}{ Trace element concentration } \\
\hline & & Maximum & Minimum & Median \\
\hline Specific conductance (umho) & 10 & 290 & 111 & 203 \\
\hline $\mathrm{pH}$ (units) & 10 & 7.9 & 7.1 & 7.5 \\
\hline Temperature $\left({ }^{\circ} \mathrm{C}\right)$ & 9 & 12.3 & 8.0 & 10.8 \\
\hline Fecal-coliform bacteria (col/100 mL) & 4 & $<1$ & $<1$ & $<1$ \\
\hline Hardness (as $\mathrm{CaCO}_{3}$ ) & 10 & 107 & 46 & 86 \\
\hline Hardness, noncarbonate (as $\mathrm{CaCO}_{3}$ ) & 10 & 9 & 0 & .5 \\
\hline Calcium, dissolved & 9 & 33 & 14 & 23 \\
\hline Magnesium, dissolved & 9 & 7.1 & 1 & 4.4 \\
\hline Sodium, dissolved & 8 & 6.6 & 1.9 & 4.5 \\
\hline Sodium-adsorption ratio & 8 & .3 & .1 & .2 \\
\hline Potassium, dissolved & 8 & 1.4 & .5 & 1.1 \\
\hline Bicarbonate & 10 & 134 & 62 & 104 \\
\hline Carbonate & 10 & 0 & 0 & 0 \\
\hline Alkal inity (as $\mathrm{CaCO}_{3}$ ) & 10 & 110 & 51 & 85 \\
\hline Sulfate, dissolved & 8 & 17 & 3.7 & 10.5 \\
\hline Chloride, dissolved & 9 & 1.3 & .0 & .8 \\
\hline Fluoride, dissolved & 8 & .2 & .0 & .1 \\
\hline Silica, dissolved (as $\mathrm{SiO}_{2}$ ) & 8 & 15 & 9 & 13 \\
\hline Solids, dissolved (residue at $180^{\circ} \mathrm{C}$ ) & 3 & 138 & 65 & 127 \\
\hline Solids, dissolved (sum of constituents) & 8 & 142 & 66 & 120 \\
\hline Nitrate (as N) & 8 & .85 & .09 & .54 \\
\hline Iron, total recoverable (ug/L) & 3 & 20 & 20 & 20 \\
\hline Iron, dissolved $(u g / L)$ & 5 & 10 & 0 & 0 \\
\hline Manganese, total recoverable (ug/L) & 1 & $<20$ & $<20$ & $<20$ \\
\hline Manganese, dissolved (ug/L) & 5 & 2 & 1 & $<1$ \\
\hline
\end{tabular}


TABLE 30.--Trace-element concentrations in ground-water samples from the Methow Region

\begin{tabular}{|c|c|c|c|c|c|c|c|c|}
\hline $\begin{array}{l}\text { LOCAL } \\
\text { IDENT - } \\
\text { I- } \\
\text { FIER }\end{array}$ & $\begin{array}{c}\text { UATE } \\
\text { OF } \\
\text { SAMPLE }\end{array}$ & $\begin{array}{l}\text { ALUM- } \\
\text { INUM, } \\
\text { TOTAL } \\
\text { RECOV- } \\
\text { ERABLE } \\
\text { (UG/L } \\
\text { AS AL) }\end{array}$ & $\begin{array}{l}\text { ALUM- } \\
\text { INUM. } \\
\text { DIS- } \\
\text { SOLVED } \\
\text { (UG/L } \\
\text { AS AL) }\end{array}$ & $\begin{array}{c}\text { ARSENIC } \\
\text { OIS- } \\
\text { SOLVED } \\
\text { (UG/L } \\
\text { AS AS) }\end{array}$ & $\begin{array}{l}\text { BARIUM, } \\
\text { DIS- } \\
\text { SOLVED } \\
\text { (UG/L } \\
\text { AS BA) }\end{array}$ & $\begin{array}{l}\text { CADMIUM } \\
\text { DIS- } \\
\text { SOLVED } \\
\text { (UG/L } \\
\text { AS CD) }\end{array}$ & $\begin{array}{l}\text { CHRO- } \\
\text { MIUM, } \\
\text { OIS- } \\
\text { SOLVED } \\
\text { IUG/L } \\
\text { AS CR! }\end{array}$ & $\begin{array}{l}\text { COPPER, } \\
\text { DIS- } \\
\text { SOLVED } \\
\text { (UG/L } \\
\text { AS CU) }\end{array}$ \\
\hline & & \multicolumn{7}{|c|}{ Okanogan County } \\
\hline $\begin{array}{l}23 E-06 K 01 \\
22 E-17001\end{array}$ & $\begin{array}{l}70-11-12 \\
79-07-09\end{array}$ & $\begin{array}{l}70 \\
--\end{array}$ & $-\overline{0}$ & -- & $\overline{10}$ & -- & $\begin{array}{r}<30 \\
0\end{array}$ & $\begin{array}{r}<50 \\
0\end{array}$ \\
\hline
\end{tabular}

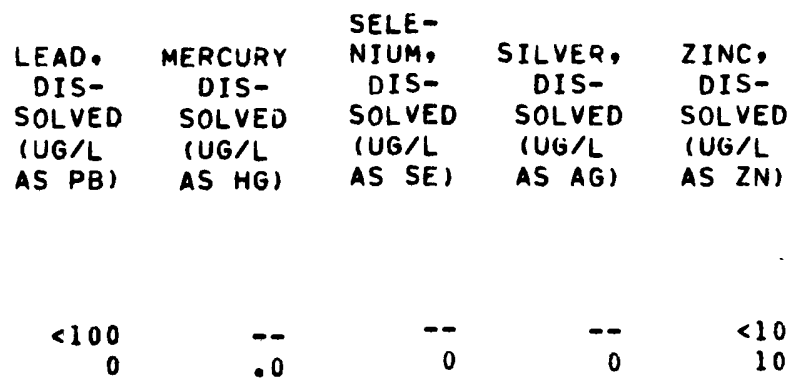


TABLE 31.--Physical, biological, and major chemical-constituent data for ground-water samples from the Chelan Region

\begin{tabular}{|c|c|c|c|c|c|c|c|c|c|c|}
\hline & & & & ELEV. & $\begin{array}{l}\text { SPE- } \\
\text { CIFIC }\end{array}$ & & & $\begin{array}{l}\text { COLI - } \\
\text { FORM. }\end{array}$ & & HARO - \\
\hline $\begin{array}{l}\text { LOCAL } \\
\text { IDENT- } \\
\text { I- } \\
\text { FIER }\end{array}$ & $\begin{array}{l}\text { GEO- } \\
\text { LOGIC } \\
\text { UNIT }\end{array}$ & $\begin{array}{l}\text { DATE } \\
\text { OF } \\
\text { SAMPLE }\end{array}$ & $\begin{array}{l}\text { UEPTH } \\
\text { OF } \\
\text { WELL, } \\
\text { TOTAL } \\
\text { (FEET) }\end{array}$ & $\begin{array}{c}\text { OF LANO } \\
\text { SURFACE } \\
\text { OATUM } \\
\text { (FT. } \\
\text { NGVO) }\end{array}$ & $\begin{array}{l}\text { CON- } \\
\text { OUCT- } \\
\text { ANCE } \\
\text { (MICHO- } \\
\text { MHOS) }\end{array}$ & $\begin{array}{c}\text { PH } \\
\text { FIELD } \\
\text { (UNITS) }\end{array}$ & $\begin{array}{l}\text { TEMPER- } \\
\text { ATURE, } \\
\text { WATER } \\
\text { (OEG C) }\end{array}$ & $\begin{array}{l}\text { FECAL, } \\
0.7 \\
\text { UH-MF } \\
(\text { COLS. } \\
100 \mathrm{ML})\end{array}$ & $\begin{array}{l}\text { HARD- } \\
\text { NESS } \\
\text { (MG/L } \\
\text { AS } \\
\text { CACO3) }\end{array}$ & $\begin{array}{l}\text { NESS } \\
\text { NONCAR- } \\
\text { BONATE } \\
\text { (MG/L } \\
\text { CACO3) }\end{array}$ \\
\hline
\end{tabular}

28/22E-21J01

28/22E-28日0l

$28 / 22 E-32 P 01$
$-71-10-20 \quad 82 \quad 2150.00$

$=\quad 79-07-24 \quad 65 \quad 2050.00$

HILGLV 79-07-11 $93 \quad 1200.00$

1200.00
MAGNE-

SIUM,

DIS-

SOLVED

IMG/L

AS $M G$ )

\section{Chelan County}

CALCIUM
DIS-
SOLVED
(MG/L
AS CA)

SOD IUM, DIS-

SOLVED

(MG/L

AS NA)

750
610
560

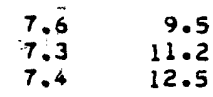

$<-$

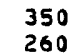

260

140

0

SOOIUM
SOR-
TION-
RATIO

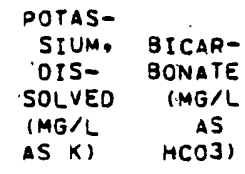
CAR-
BONATE
IMG/L
AS $\mathrm{CO} 3$ ?

\begin{tabular}{|c|c|c|c|c|c|c|c|c|}
\hline $\begin{array}{l}86 \\
60 \\
33\end{array}$ & $\begin{array}{l}34 \\
27 \\
14\end{array}$ & $\begin{array}{l}30 \\
25 \\
69\end{array}$ & $\begin{array}{r}.7 \\
: 7 \\
2: 5\end{array}$ & $\begin{array}{r}2.6 \\
2.8 \\
3.2 \\
.\end{array}$ & $\begin{array}{l}437 \\
354 \\
305\end{array}$ & $\begin{array}{l}0 \\
0 \\
0\end{array}$ & $\begin{array}{l}358 \\
290 \\
250\end{array}$ & $\begin{array}{l}56 \\
44 \\
30\end{array}$ \\
\hline $\begin{array}{l}\text { CHLO- } \\
\text { RIUE, } \\
\text { OIS- } \\
\text { SOLVEO } \\
\text { (MG/L } \\
\text { AS CL) }\end{array}$ & $\begin{array}{l}\text { FLUO- } \\
\text { RIOE, } \\
\text { OIS- } \\
\text { SOLVED } \\
\text { IMG/L } \\
\text { AS FI) }\end{array}$ & $\begin{array}{l}\text { SILICA, } \\
\text { DIS- } \\
\text { SOLVED } \\
\text { (MG/L } \\
\text { AS } \\
\text { SIOL) }\end{array}$ & $\begin{array}{l}\text { SOLIDS, } \\
\text { RESIDUE } \\
\text { AT I8O } \\
\text { DEG. C } \\
\text { DIS- } \\
\text { SOLVED } \\
\text { (MG/L) }\end{array}$ & $\begin{array}{l}\text { SOLIOS: } \\
\text { SUM OF } \\
\text { CONSTI- } \\
\text { TUENTS. } \\
\text { DIS- } \\
\text { SOLVED } \\
\text { (MG/L) }\end{array}$ & $\begin{array}{l}\text { NITRO- } \\
\text { GEN, } \\
\text { NOZ+NO3 } \\
\text { TOTAL } \\
\text { (MG/L } \\
\text { AS NI }\end{array}$ & $\begin{array}{c}\text { NITRO- } \\
\text { GEN. } \\
\text { NOZ+NO3 } \\
\text { DIS- } \\
\text { SOLVED } \\
\text { (MG/L } \\
\text { AS NI }\end{array}$ & $\begin{array}{l}\text { IRON. } \\
\text { TOTAL } \\
\text { RECOV- } \\
\text { ERAOLE } \\
\text { (UG/L } \\
\text { AS FE) }\end{array}$ & $\begin{array}{l}\text { IRON. } \\
\text { DIS- } \\
\text { SOLVED } \\
\text { (UG/L } \\
\text { AS FE) }\end{array}$ \\
\hline
\end{tabular}

\begin{tabular}{|c|c|c|c|c|c|c|c|}
\hline $\begin{array}{l}8.1 \\
6.0 \\
3.3\end{array}$ & $\begin{array}{l}.4 \\
.2 \\
.4\end{array}$ & $\begin{array}{l}44 \\
38 \\
31\end{array}$ & $\begin{array}{r}490 \\
=-\end{array}$ & $\begin{array}{l}480 \\
381 \\
349\end{array}$ & 1.0 & 3.79 & $\begin{array}{l}40 \\
-\end{array}$ \\
\hline $\begin{array}{l}\text { MANGA- } \\
\text { NESE. } \\
\text { TOTAL } \\
\text { RECOV- } \\
\text { ERABLE } \\
\text { (UG/L } \\
\text { AS MN) }\end{array}$ & $\begin{array}{l}\text { MANGA- } \\
\text { NESE, } \\
\text { UIS- } \\
\text { SOLVED } \\
\text { (UG/L } \\
\text { AS MN) }\end{array}$ & $\begin{array}{l}\text { ALUM- } \\
\text { INUM. } \\
\text { TOTAL } \\
\text { RECOV- } \\
\text { ERABLE } \\
\text { (UG/L } \\
\text { AS AL) }\end{array}$ & $\begin{array}{l}\text { CHRO- } \\
\text { MIUM, } \\
\text { DIS- } \\
\text { SOLVED } \\
\text { (UG/L } \\
\text { AS CA) }\end{array}$ & $\begin{array}{l}\text { COPPER, } \\
\text { DIS- } \\
\text { SOLVED } \\
\text { (UG/L } \\
\text { AS CU) }\end{array}$ & $\begin{array}{l}\text { LEAD. } \\
\text { DIS- } \\
\text { SOLVED } \\
\text { (UG/L } \\
\text { AS PH) }\end{array}$ & $\begin{array}{l}\text { ZINC, } \\
\text { DIS- } \\
\text { SOLVED } \\
\text { (UG/L } \\
\text { AS ZN) }\end{array}$ & \\
\hline
\end{tabular}

$\begin{array}{lllll}<20 & -0 & 40<30<50<100<10 \\ -0 & <1\end{array}$


TABLE 32.--Physical, biological, and major chemical-constituent data for ground-water samples from the Entiat subregion

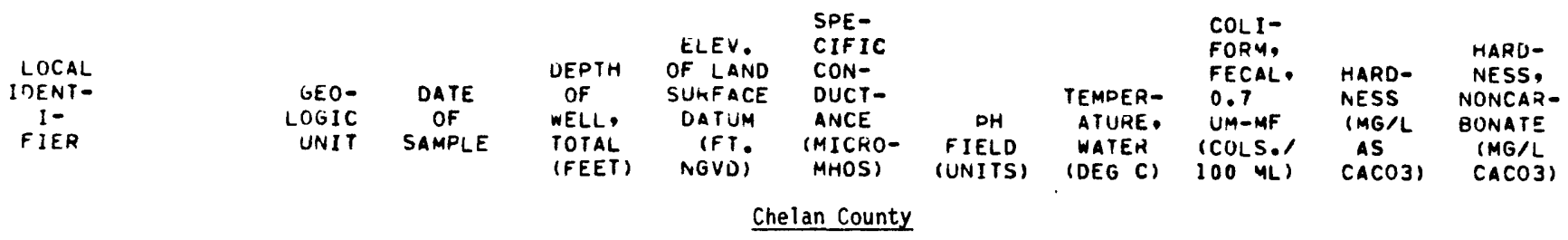

$25 / 20 E-03001$
$25 / 20 E-14001$

$26 / 20 E-20001$

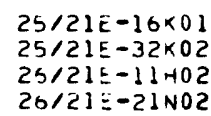

LOCAL
IDENT-
I-

FIEH

$25 / 20 E-03001$ $25 / C \cap E-14001$ $25 / 20 t-20 P O 1$

$25 / 21 E-16 K 01$ 25/टlE-32K02 26/21E-11H02 $26 / 21 E-21 N O 2$

LOCAL

IDENT-

I-

FIER $\begin{array}{ll}110 A L V M & 79-07-12 \\ 11 \text { ULLVM } & 74-07-24\end{array}$

110ALVM 79-07-12

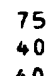

1013.00
890.00
1235.00

165
145

7.1

7.5

9.4
11.8

--

13.1

Douglas County

112GLCV 79-07-11

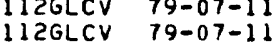

$112 G L C V$ 79-07-11

$112 G L C V$ 71-05-25

$112 G L C V \quad 71-10-20$

115

97
164

159

159

$112 G L C V$ 74-07-11

159

700.00

700.00
720.00

720.00

801.00

801.00

510
725
255
399
499
520

$7 \cdot 3$

7.3
7.7

8.2

13.1

12.2

13.7

13.6

801.00

520

7.6

14.0

SODIUM POTAS-

$\begin{array}{lll} & \text { MAGNE- } \\ \text { CALCIUM } & \text { SIUM, } & \text { SODIUM, } \\ \text { DIS- } & \text { DIS- } & \text { UIS- } \\ \text { SOLVEO } & \text { SOLVED } & \text { SOLVED } \\ \text { (MG/L } & \text { (MG/L } & \text { (MG/L } \\ \text { AS CA) } & \text { AS MS) } & \text { AS NA) }\end{array}$

SORP-

PIUS-

DIS-

TION

RATIO

SOLVED

(MG/L

AS $K$ )

BICAR-
BONATE

IMGIL

$A S$
HCOS

Chelan County

.3
.2
.2

1.7
2.3
2.4

91
62
146

CAR-
BONATE

(MG/L

(MG/L

ALKA-

LINITY

IMG/L

AS

(ACO3)

$<1$

$\begin{array}{ll}- & 270 \\ 1 & 300 \\ - & 110 \\ -\quad 170 \\ -\quad 220 \\ -\quad 250\end{array}$

40
30
19
48
-0

110
CHLO$\begin{array}{cl}\text { SULFATE } & \text { RIDE, } \\ \text { DIS- } & \text { DIS- } \\ \text { SOLVED } & \text { SOLVED }\end{array}$ $\begin{array}{ll}\text { SULFATE } & \text { RIDE, } \\ \text { DIS- } & \text { DIS- } \\ \text { SOLVED } & \text { SOLVED }\end{array}$ IMG/L (MGLL AS SO4)
2.4 1.0

\section{Douglas County}

$\begin{array}{lll}82 & 15 & 8.5 \\ 79 & 25 & 18 \\ 32 & 7.2 & 5.7 \\ 48 & 12 & 12 \\ 68 & 13 & -- \\ 70 & 14 & 14\end{array}$

$\begin{array}{rr}2.3 & 280 \\ 3.8 & 329 \\ 1.9 & 111 \\ 3.6 & 148 \\ -0 & -0 \\ 4.2 & 171\end{array}$

4.2

171
SOLIUS,

$\begin{array}{ll}\text { FLUO- } & \text { SILICA, } \\ \text { HIDE, } & \text { DIS- } \\ \text { DIS- } & \text { SOLVED } \\ \text { SULVEU } & \text { (MG/L } \\ \text { (MG/L } & \text { AS } \\ \text { ASF) } & \text { SIOL) }\end{array}$
RESIDUE AT 1 OO DEG. C DISSOLVED (MG/L)
SOLIDS, SUM OF CONSTITUENTS, UISSULVED (MG/L)

$\begin{array}{cc}\text { NITRO- } \\ \text { NITRO- } & \text { GEN, } \\ \text { GEN, } & \text { NOZ+NO3 } \\ \text { NOZ+NO3 } & \text { DIS- } \\ \text { TOTAL } & \text { SOLVED } \\ \text { (MGNL } & \text { (MGLL } \\ \text { AS N) } & \text { AS N) }\end{array}$

IRON,

TOTAL

RECOV-

ERABLE

(UG/L

AS FE)
0
0
0

0
75
51
120

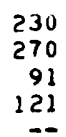

230
270
91
121
-0

140

120

Chelan County

$25 / 20 E-03001$ $25 / \angle 0 E-14001$ $26 / 20 E-20001$

25/21E-16K0 $25 / 21 t-32 \times 02$ $26 / 21 E-11402$ $24 / 21 t-21 N 02$

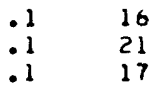

$\because$

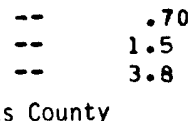

Douglas County

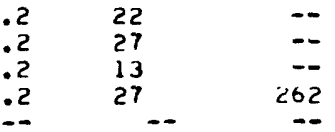

. 321

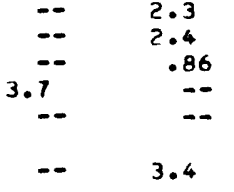

$\because$

IHON.

DIS-

SOLVED

(UG/L

AS FE)
MANGANESE, TOTAL RECOV-

ERABLE (UG/L AS MN) 
TABLE 33.--Summary of physical, biological, and major chemical-constituent data for ground-water samples from the Entiat subregion

[Values in milligrams per liter except as indicated umho, micromho; col $/ 100 \mathrm{~mL}$, colonies per $100 \mathrm{milliliter;}$ $\mathrm{ug} / \mathrm{L}$, microgram per liter]

\begin{tabular}{|c|c|c|c|c|}
\hline Constituent & $\begin{array}{l}\text { Number of } \\
\text { sample sites }\end{array}$ & $\frac{\text { Trace eles }}{\text { Maximum }}$ & $\begin{array}{l}\text { ent conce } \\
\text { Minimum }\end{array}$ & $\frac{\text { itration }}{\text { Median }}$ \\
\hline Specific conductance (umho) & 7 & 725 & 145 & 275 \\
\hline $\mathrm{pH}$ (units) & 7 & 8.2 & 7.1 & 7.3 \\
\hline Temperature $\left({ }^{\circ} \mathrm{C}\right)$ & 7 & 14.0 & 9.4 & 12.2 \\
\hline Fecal-coliform bacteria (col/100 mL) & 2 & $<1$ & $<1$ & $<1$ \\
\hline Hardness (as $\mathrm{CaCO}_{3}$ ) & 7 & 300 & 60 & 120 \\
\hline Hardness, noncarbonate (as $\mathrm{CaCO}_{3}$ ) & 7 & 110 & 0 & 19 \\
\hline Calcium, dissoived & 7 & 82 & 17 & 32 \\
\hline Magnesium, dissolved & 7 & 25 & 4.3 & 12 \\
\hline Sodium, dissolved & 7 & 18 & 4.3 & 6.2 \\
\hline Sodium-adsorption ratio & 7 & .5 & .2 & .2 \\
\hline Potassium, dissolved & 7 & 4.2 & 1.7 & 2.3 \\
\hline Bicarbonate & 7 & 329 & 62 & 146 \\
\hline Carbonate & 7 & 0 & 0 & 0 \\
\hline Alkalinity $\left(\right.$ as $\left.\mathrm{CaCO}_{3}\right)$ & 7 & 270 & 51 & 120 \\
\hline Sulfate, dissolved & 7 & 120 & 5.6 & 24 \\
\hline Chioride, dissolved & 7 & 7.9 & 1.0 & 2.4 \\
\hline Fluoride, dissolved & 7 & .3 & .1 & .2 \\
\hline Silica, dissolved (as $\mathrm{SiO}_{2}$ ) & 7 & 27 & 13 & 21 \\
\hline Solids, dissolved (residue at $180^{\circ} \mathrm{C}$ ) & 1 & 262 & 262 & 262 \\
\hline Solids, dissolved (sum of constituents) & 7 & 394 & 97 & 163 \\
\hline Nitrate (as $N$ ) & 7 & 3.8 & .7 & 2.3 \\
\hline Iron, total recoverable (ug/L) & 1 & 10 & 10 & 10 \\
\hline Iron, dissolved (ug/L) & 7 & 10 & 0 & 0 \\
\hline Manganese, total recoverable $(\mathrm{ug} / \mathrm{L})$ & 1 & 20 & 20 & 20 \\
\hline Manganese, dissolved (ug/L) & 7 & $<1$ & $<1$ & $<1$ \\
\hline
\end{tabular}


TABLE 34.--Trace-element concentrations in ground-water samples from the Entiat subregion

\begin{tabular}{|c|c|c|c|c|c|c|c|}
\hline $\begin{array}{l}\text { LOCAL } \\
\text { IDENT- } \\
\text { I- } \\
\text { FIER }\end{array}$ & $\begin{array}{c}\text { DATE } \\
\text { OF } \\
\text { SAMPLE }\end{array}$ & $\begin{array}{l}\text { ALUM- } \\
\text { INUM, } \\
\text { TOTAL } \\
\text { RECOV- } \\
\text { ERABLE } \\
\text { (UG/L } \\
\text { AS AL) }\end{array}$ & $\begin{array}{l}\text { ALUM- } \\
\text { INUM, } \\
\text { OIS- } \\
\text { SOLVED } \\
\text { (UG/L } \\
\text { AS AL) }\end{array}$ & $\begin{array}{c}\text { ARSENIC } \\
\text { DIS- } \\
\text { SULVED } \\
\text { (UG/L } \\
\text { AS AS) }\end{array}$ & $\begin{array}{l}\text { BARIUM, } \\
\text { DIS- } \\
\text { SOLVED } \\
\text { (UG/L } \\
\text { AS BA) }\end{array}$ & $\begin{array}{l}\text { CADMIUM } \\
\text { DIS- } \\
\text { SOLVED } \\
\text { (UG/L } \\
\text { AS CDI }\end{array}$ & $\begin{array}{l}\text { CHRO- } \\
\text { MIUM, } \\
\text { DIS- } \\
\text { SOLVED } \\
\text { IUG/L } \\
\text { AS CR }\end{array}$ \\
\hline $25 / 20 E-03001$ & $79-07-12$ & -- & 0 & 0 & 30 & $<1$ & 0 \\
\hline \multirow[t]{4}{*}{$26 / 21 E-21 N 02$} & $\begin{array}{l}71-05-25 \\
79-07-11\end{array}$ & $<10$ & -- & -- & $\overline{80}$ & $-\infty$ & $\begin{array}{r}<30 \\
0\end{array}$ \\
\hline & & $\begin{array}{l}\text { COPPER, } \\
\text { DIS- } \\
\text { SOLVED } \\
\text { (UG/L } \\
\text { AS CU) }\end{array}$ & $\begin{array}{l}\text { LEAD, } \\
\text { DIS- } \\
\text { SOLVED } \\
\text { (UG/L } \\
\text { AS PB) }\end{array}$ & $\begin{array}{c}\text { MERCURY } \\
\text { DIS- } \\
\text { SOLVED } \\
\text { (UG/L } \\
\text { AS HG) }\end{array}$ & $\begin{array}{l}\text { SELE- } \\
\text { NIUM, } \\
\text { DIS- } \\
\text { SOLVED } \\
\text { (UG/L } \\
\text { AS SE) }\end{array}$ & $\begin{array}{l}\text { SILVER, } \\
\text { DIS- } \\
\text { SOLVED } \\
\text { (UG/L } \\
\text { AS AG) }\end{array}$ & $\begin{array}{l}\text { ZINC, } \\
\text { OIS- } \\
\text { SOLVED } \\
\text { (UG/L } \\
\text { AS ZN) }\end{array}$ \\
\hline & & 2 & 0 & .0 & 0 & 0 & $<3$ \\
\hline & & $\begin{array}{r}<50 \\
2\end{array}$ & $\begin{array}{r}<100 \\
0\end{array}$ & $-\overline{0}$ & $-\frac{-}{1}$ & -- & $\begin{array}{r}<10 \\
<3\end{array}$ \\
\hline
\end{tabular}




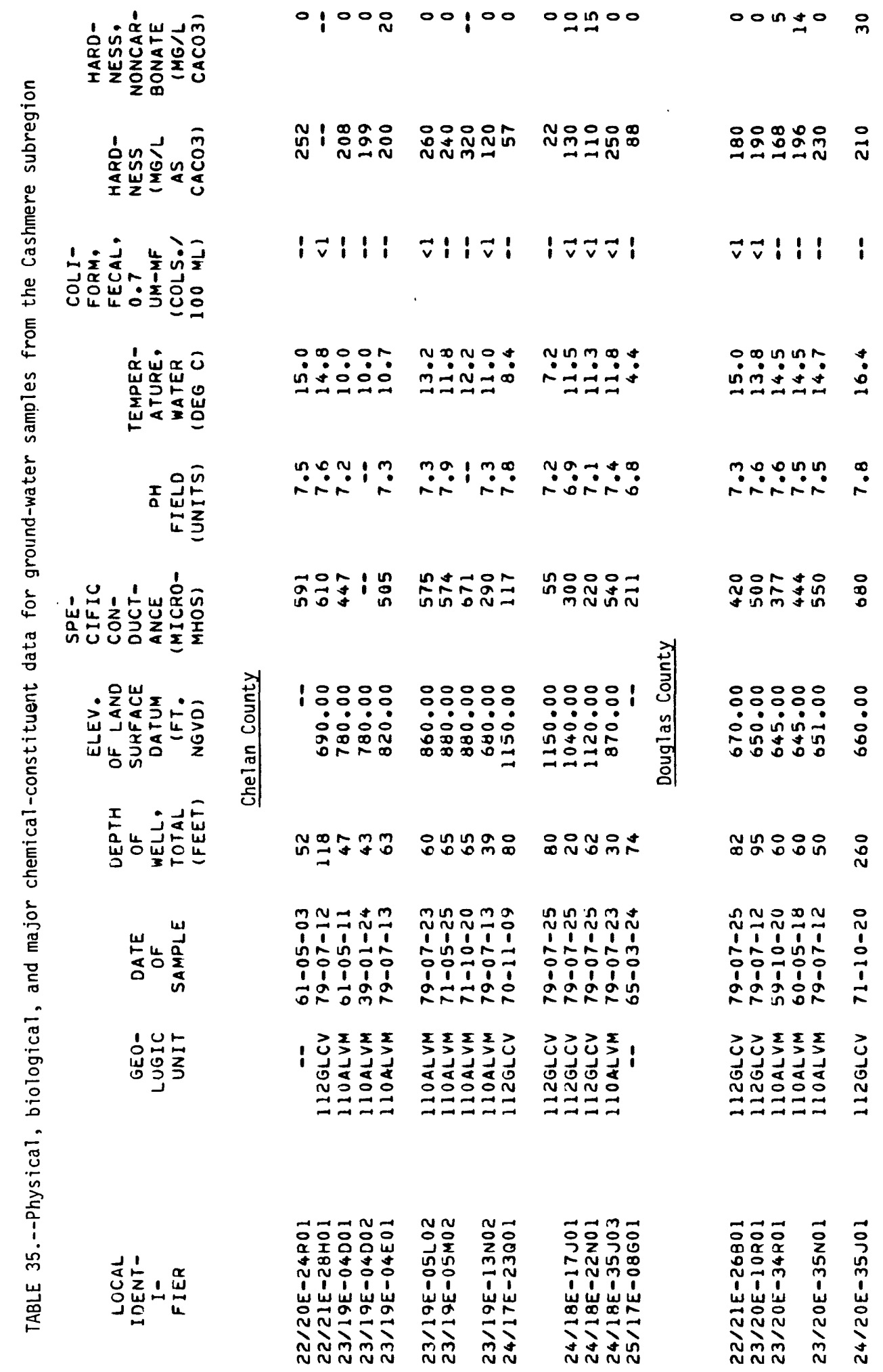




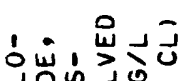

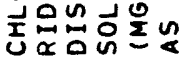

崖, 品势

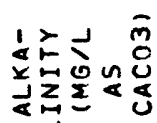

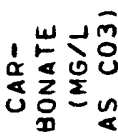

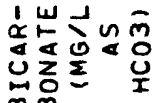

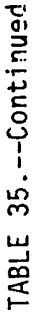

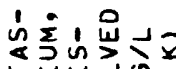

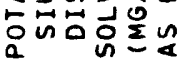

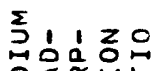

品实高

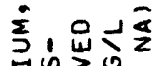

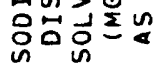

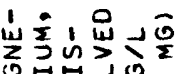

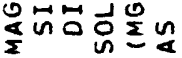

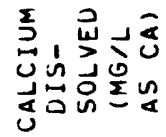

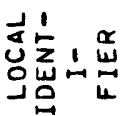

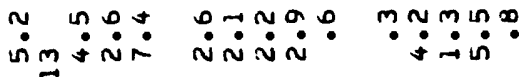

ษ̊ming

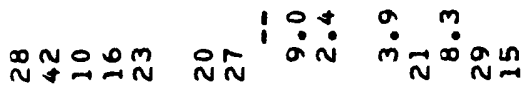

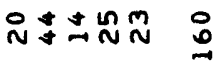

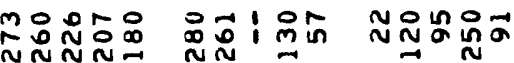

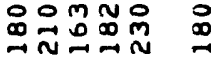

000000

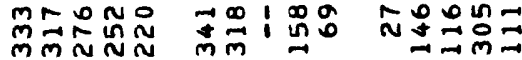

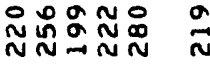

可

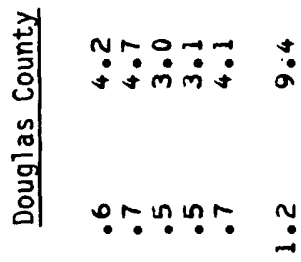

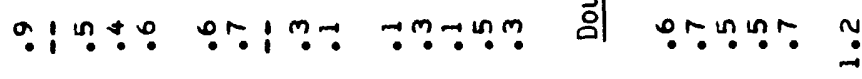

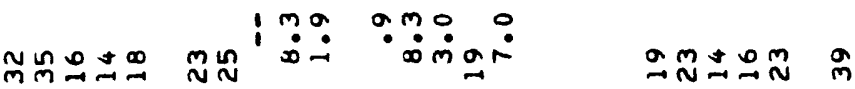

゚゙

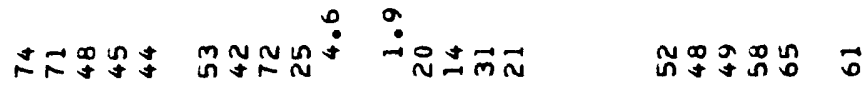

\begin{tabular}{|c|c|c|c|c|}
\hline 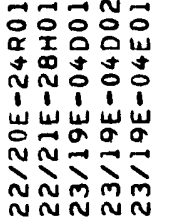 & 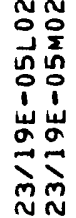 & 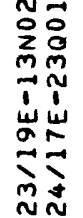 & 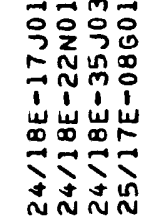 & 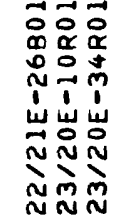 \\
\hline
\end{tabular}


这岕的崖这

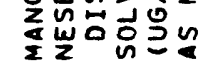

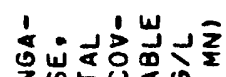

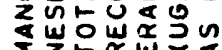

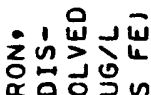

唫昰

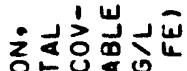

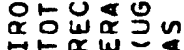

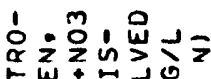

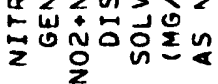

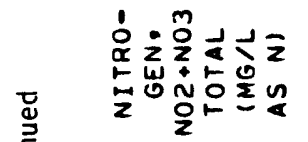

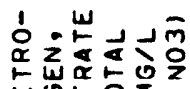

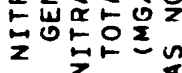

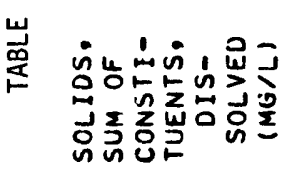

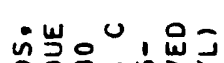

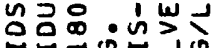

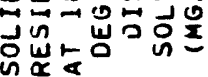

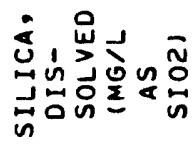

’的出号

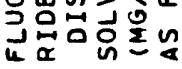

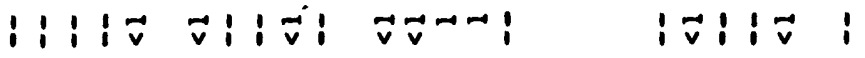

I: I: I I원 i

i : i i :

i: $1^{0} 0: 101$ 0000!

1운우

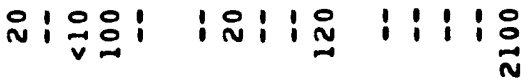

! I윰영

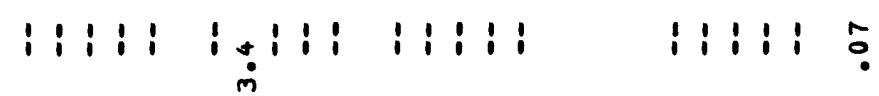

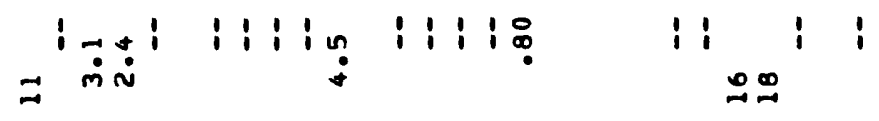

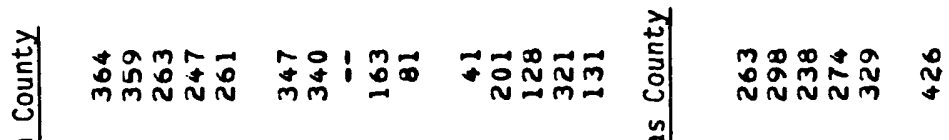

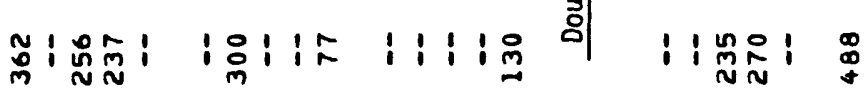

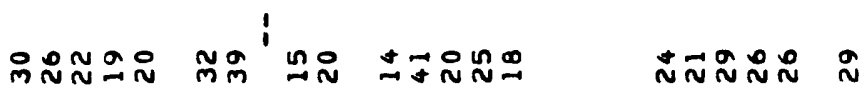

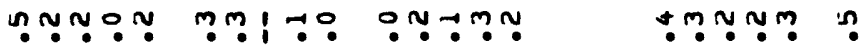

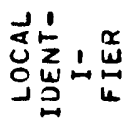

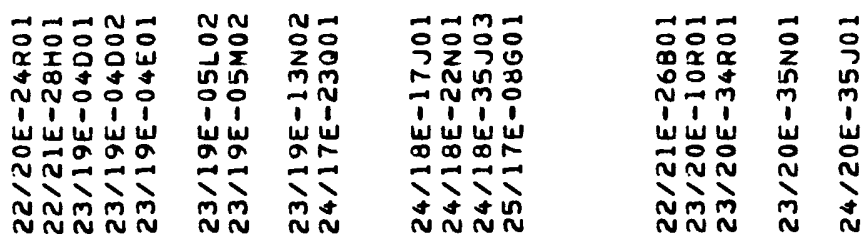


TABLE 36.--Summary of physical, biological, and major chemical-constituent data for ground-water samples from the Cashmere subregion

[Values in milligrams per liter except as indicated umho, micromho; col/100 mL, colonies per 100 milliliter; ug/L, microgram per liter]

\begin{tabular}{|c|c|c|c|c|}
\hline \multirow[b]{2}{*}{ Constituent } & \multirow{2}{*}{$\begin{array}{l}\text { Number of } \\
\text { sample sites }\end{array}$} & \multicolumn{3}{|c|}{ Trace element concentration } \\
\hline & & Maximum & Minimum & Median \\
\hline Specific conductance (umho) & 17 & 680 & 55 & 500 \\
\hline $\mathrm{pH}$ (units) & 17 & 7.9 & 6.8 & 7.4 \\
\hline Temperature $\left({ }^{\circ} \mathrm{C}\right)$ & 18 & 16.4 & 4.4 & 11.9 \\
\hline Fecal-coliform bacteria (col/100 mL) & 8 & $<1$ & $<1$ & $<1$ \\
\hline Hardness (as $\mathrm{CaCO}_{3}$ ) & 17 & 320 & 22 & 199 \\
\hline Hardness, noncarbonate (as $\mathrm{CaCO}_{3}$ ) & 17 & 30 & 0 & 0 \\
\hline Calcium, dissolved & 18 & 74 & 1.9 & 48 \\
\hline Magnesium, dissolved & 17 & 41 & 4.3 & 17 \\
\hline Sodium, dissolved & 18 & 39 & .9 & 18.5 \\
\hline Sodium-adsorption ratio & 17 & 1.2 & .1 & .5 \\
\hline Potassium, dissolved & 18 & 9.4 & 1.4 & 3.2 \\
\hline Bicarbonate & 18 & 341 & 27 & 236 \\
\hline Carbonate & 18 & 0 & 0 & 0 \\
\hline Alkal inity $\left(\right.$ as $\mathrm{CaCO}_{3}$ ) & 18 & 280 & 22 & 194 \\
\hline Sulfate, dissolved & 18 & 160 & 2.4 & 20.5 \\
\hline Chloride, dissolved & 14 & 14 & .3 & 4.2 \\
\hline Fluoride, dissolved & 18 & .5 & .0 & .2 \\
\hline Silica, dissolved (as $\mathrm{SiO}_{2}$ ) & 18 & 41 & 14 & 24.5 \\
\hline Solids, dissolved (residue at $180^{\circ} \mathrm{C}$ ) & 8 & 488 & 77 & 254 \\
\hline Solids, dissolved (sum of constituents) & 18 & 426 & 41 & 263 \\
\hline Nitrate (as N) & 19 & 4.4 & .04 & 2.4 \\
\hline Iron, total recoverable $(u g / L)$ & 8 & 2,100 & 10 & 60 \\
\hline Iron, dissolved (ug/L) & 9 & 10 & 0 & 10 \\
\hline Manganese, total recoverable (ug/L) & 4 & 400 & 20 & 70 \\
\hline Manganese, dissolved (ug/L) & 9 & 1 & $<1$ & $<1$ \\
\hline
\end{tabular}


TABLE 37.--Trace-element concentrations in ground-water samples from the Cashmere subregion

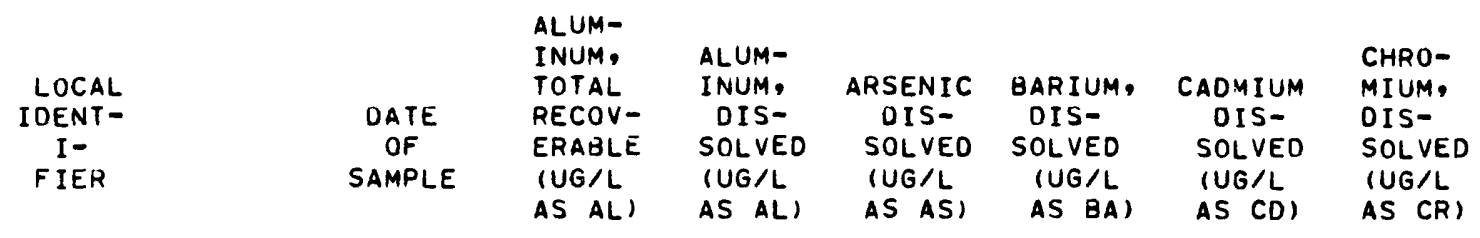

$23 / 19 E-05 M 02$
$23 / 19 E-13 N 02$
$24 / 17 E-23001$
$24 / 18 E-17 J 01$

$22 / 21 E-26801$

$24 / 20 E-35 J 01$

$$
\begin{aligned}
& 71-05-25 \\
& 79-07-13 \\
& 70-11-09 \\
& 79-07-25
\end{aligned}
$$

$79-07-25$

$71-10-20$

\section{Chelan County}

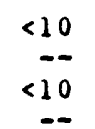

$-2$

$\frac{-2}{2}$
$\overline{70}$
20

$\begin{array}{rr}-- & <30 \\ <1 & 0 \\ -- & <30 \\ <1 & 0\end{array}$

Douglas County

$\overline{40}$

$\stackrel{0}{-}$

$\because \quad-$

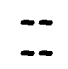

0

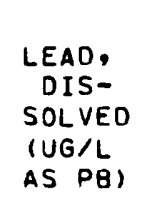
MERCURY DIS-
SOLVED
IUG/L
AS HGI

SELE-

NIUM,

OIS-

SOLVED

(UG/L

AS SE)

SILVER,

DIS-

SOLVEO

(UG/L

AS AG)

Chelan County
$<50$

100

$<50$

4

2

$$
.0
$$$$
\frac{-}{0}
$$

$--$

Douglas County

2
$<50 \quad<100$

$\because 0$

$--$

ZINC, DISSOLVED (UG/L AS ZN) 